\title{
NOVEL LOW TEMPERATURE COFIRED CERAMIC MANUFACTURING TECHNIQUES FOR A MAGNETRON FIELD EMISSION CATHODE
}

\author{
by \\ Daylon Michael Black
}

\author{
A thesis \\ submitted in partial fulfillment \\ of the requirements for the degree of \\ Master of Science in Mechanical Engineering \\ Boise State University
}

May 2019 
(C) 2019

Daylon Michael Black

ALL RIGHTS RESERVED 


\title{
DEFENSE COMMITTEE AND FINAL READING APPROVALS
}

\author{
of the thesis submitted by \\ Daylon Michael Black
}

Thesis Title: Novel Low Temperature Cofired Ceramic Manufacturing Techniques for a Magnetron Field Emission Cathode

Date of Final Oral Examination: 10 December 2018

The following individuals read and discussed the thesis submitted by student Daylon Michael Black, and they evaluated his presentation and response to questions during the final oral examination. They found that the student passed the final oral examination.

Donald Plumlee, Ph.D. Chair, Supervisory Committee

Jim Browning, Ph.D. Member, Supervisory Committee

John F. Gardner, Ph.D. Member, Supervisory Committee

The final reading approval of the thesis was granted by Donald Plumlee, Ph.D., Chair of the Supervisory Committee. The thesis was approved by the Graduate College. 
This thesis is dedicated to my parents and brother; everything I have achieved is because of them and I am forever grateful. 


\section{ACKNOWLEDGEMENTS}

I would like to thank god for giving me the knowledge, strength, and opportunity to undertake this research. This research as well as the many gifts in my life would not have been possible without his guidance.

I would also like to thank my mom, dad, and brother for all of their love and support throughout my life and especially throughout this project. They have always been their when I needed them and they have always believed in me.

I would also like to thank Courtney Smith for all of her encouragement, support, understanding, and love during this process. She has been my pillar of strength throughout the tough times and I could not have done this without her.

I would also like to thank Jason Kuwada, Jakob Smith, Austin Jones, Andrew Souza, Oliver Alvarez, Griff Allen, and Brent Johnston for all of the wisdom and encouragement throughout the years, some academia related... some not.

I also wish to give special thanks to my esteemed committee: Don Plumlee, Jim Browning, and John Gardner for their guidance and support.

A huge thank you to the CMEMS and VED laboratories at Boise State University including Theo Moodley, Zachary Taylor, Patrick Ward, Ryan Harper, Andy Yue, Tiffany Berntsen, Marcus Pearlman, Tanya Lu, and Stephen Saldivar for all of their support, knowledge, and expertise. Wakanda Forever. Additionally, I would like to thank Omar Betancourt for the facet plate laser technique procedure. 
I would also like to thank L3 Technologies who provided both the magnetron hardware and the design that was used in this research. In particular, I would like to thank Carter Armstrong, Mike Worthington, and John Cipolla for their support.

I would also like to thank DuPont, Inc for their support of the LTCC materials. Material support for this research was provided by the Air Force Office of Scientific Research under Award \#FA9550-16-1-0083. 


\begin{abstract}
Low Temperature Cofired Ceramic (LTCC) is a material system that is ideal for integrated microelectronic packaging technology, because of its rapid prototyping and easy integration of passive components such as resistors, capacitors, and conductors. LTCC's electrical properties makes it especially suitable for high frequency applications such as magnetrons. Recently, there has been an increased demand for greater power capacities which is resolved by phase locking multiple low power (inexpensive) magnetrons together to achieve the same power as one high power (expensive) magnetron. The Vacuum Electron Devices (VED) and Ceramic Micro Electrical Mechanical Systems (CMEMS) labs at Boise State University have designed a new field emission cathode for the L3 Technologies industrial magnetron that will precisely inject electrons into the system. This controllable electron injection, has been shown by simulation to decrease startup times, increase efficiency, and even allow for active phase control during oscillation. In this research, new LTCC manufacturing techniques were developed in order to fabricate the newly designed cathode. Two different cathode wrapping techniques were compared and the layer by group technique was faster, more easily aligned, and had a shorter learning curve. Two different via filling techniques were compared and the flat fill technique was slightly slower but, more repeatable and was less likely to cause electrical shorting. Two different facet plate manufacturing techniques were compared and the mill technique was more precise and extremely repeatable. Four different stands were compared and the square post stand had the best circularity and
\end{abstract}


linearity measurements of the fired cathode. Finally, the spiral embedded stripline line was prototyped and an elementary version was assembled which matched simulations. 
TABLE OF CONTENTS

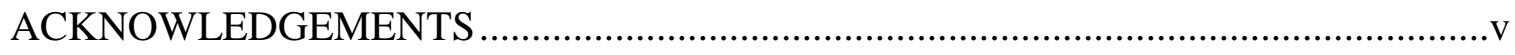

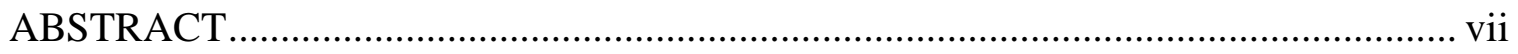

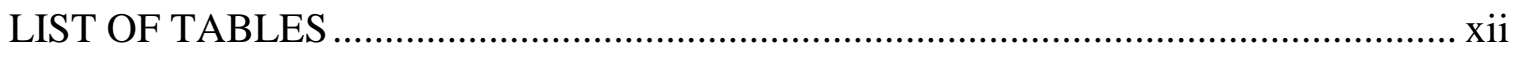

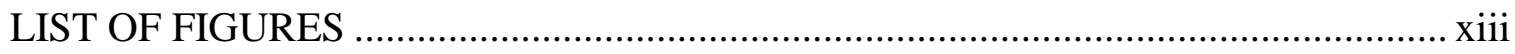

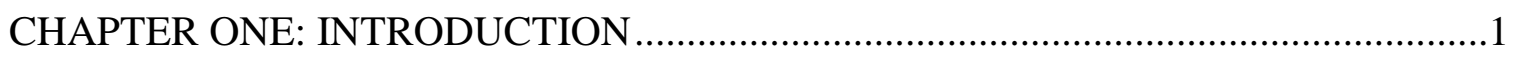

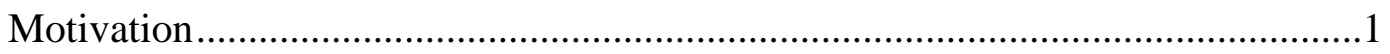

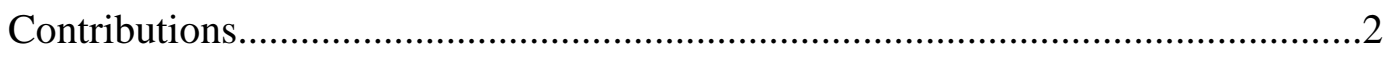

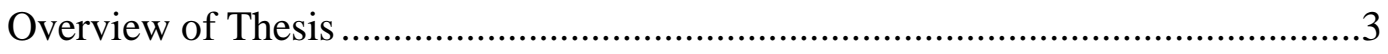

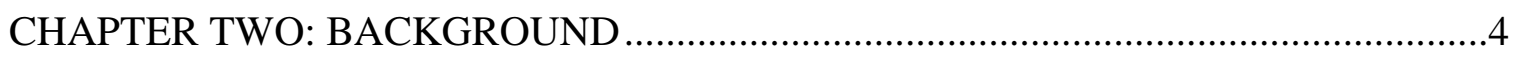

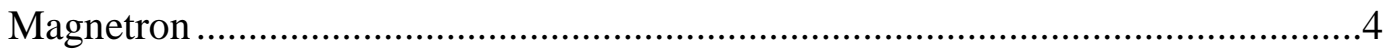

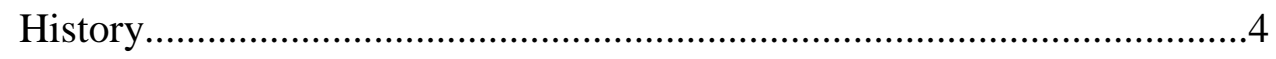

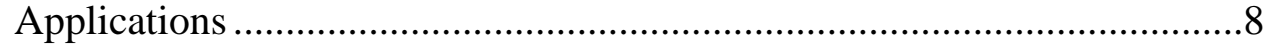

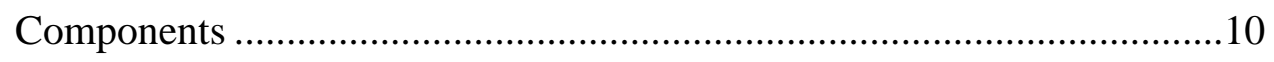

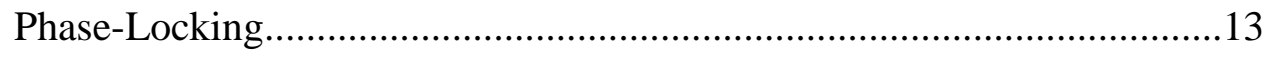

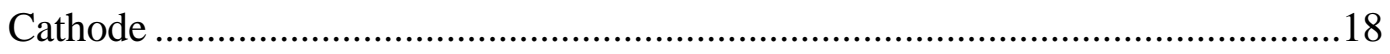

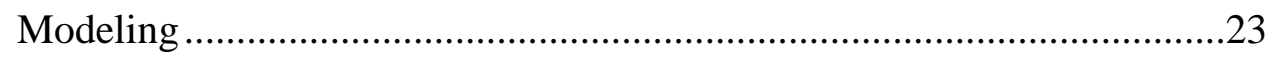

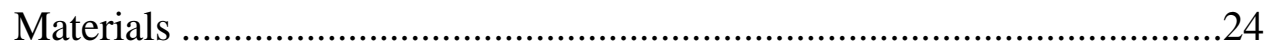

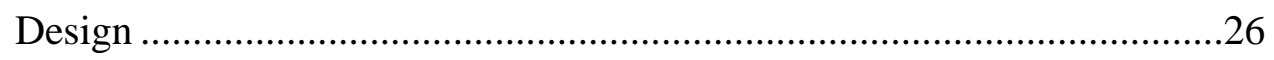

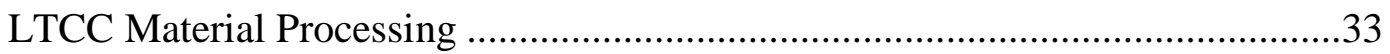




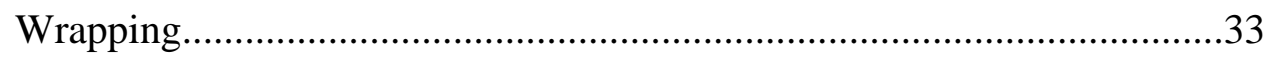

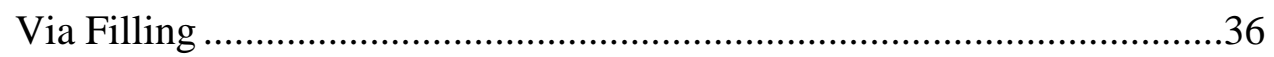

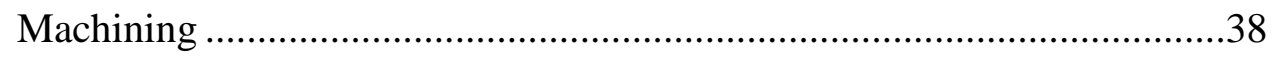

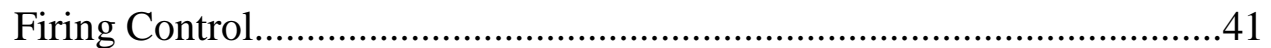

Screen Cutting ............................................................................4

CHAPTER THREE: TECHNIQUES …..........................................................47

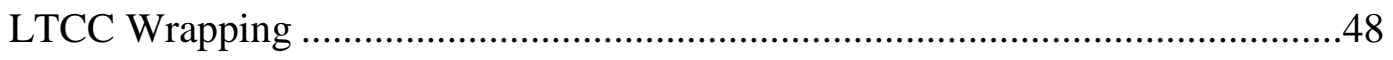

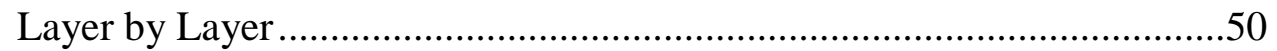

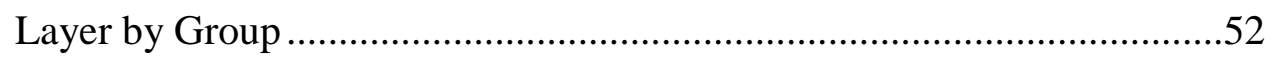

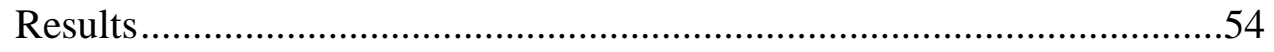

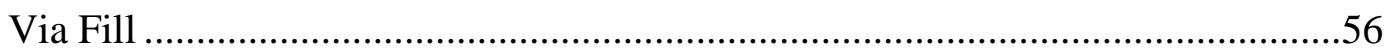

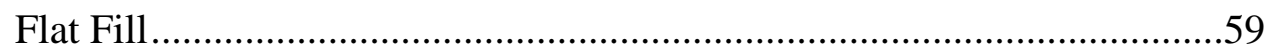

Pneumatic Pump ...........................................................................62

Clearance Hole ............................................................................66

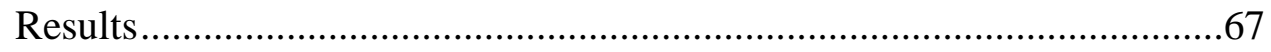

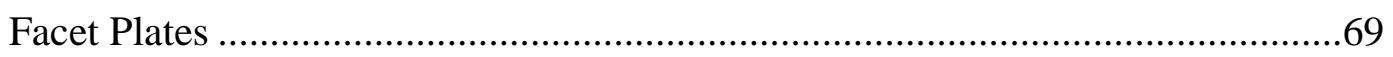

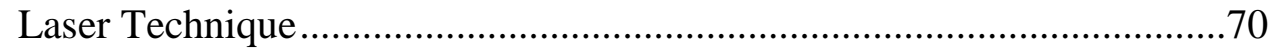

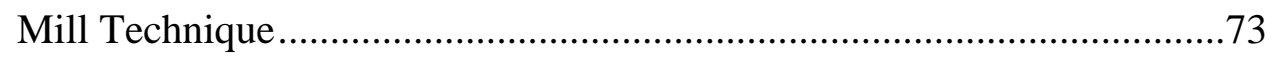

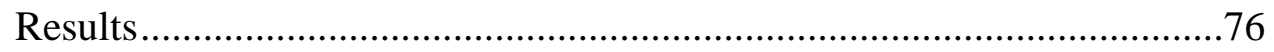

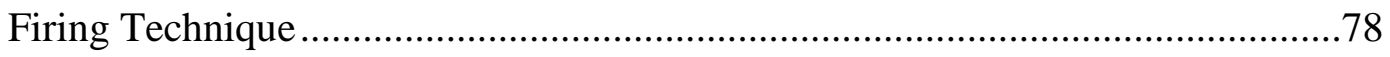

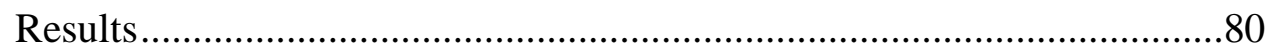

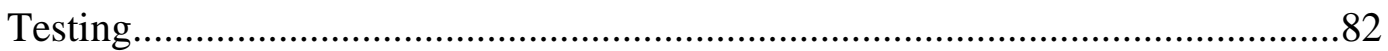

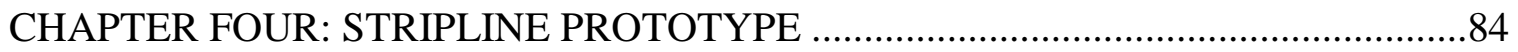




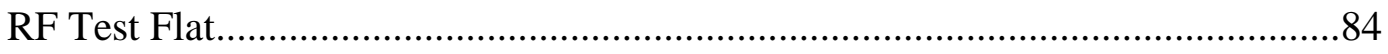

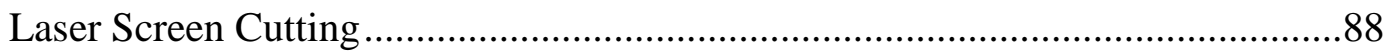

Manual Screen Printing...........................................................................90

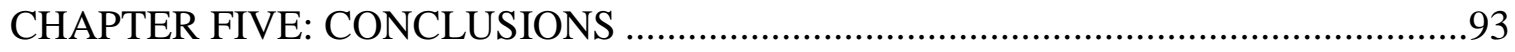

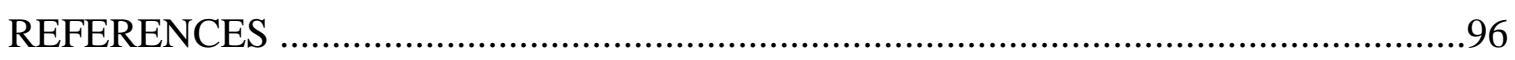

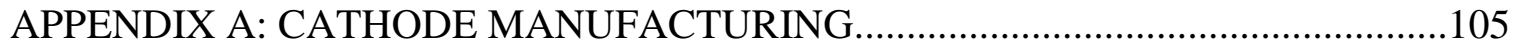

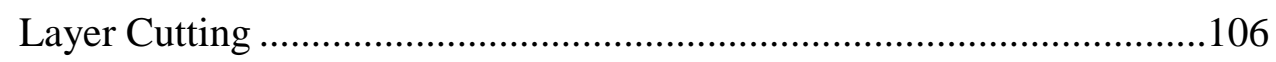

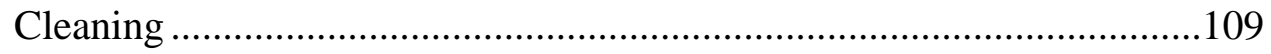

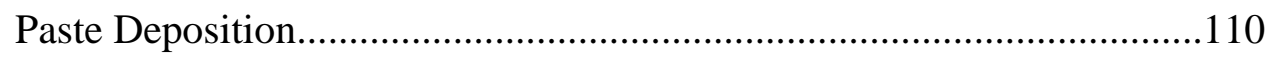

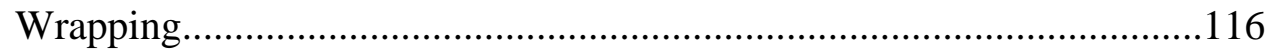

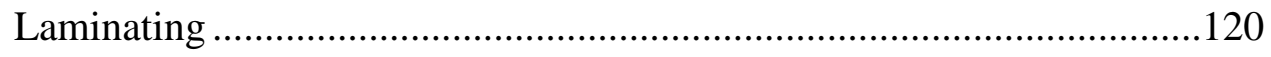

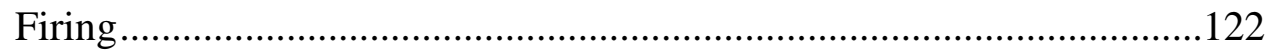

Facet Plates ......................................................................... 123

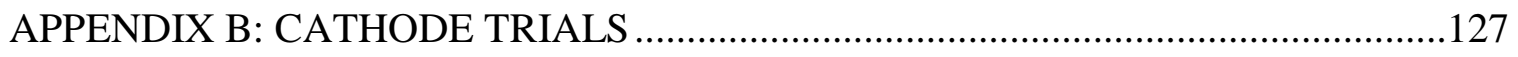

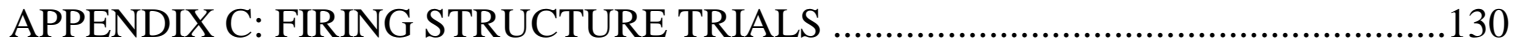

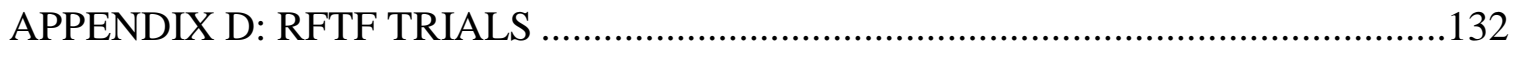

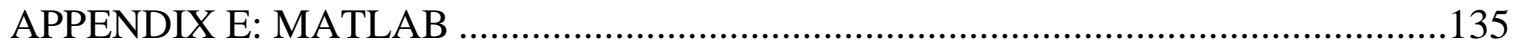




\section{LIST OF TABLES}

Table 1. VORPAL simulation magnetron dimensions [12] ................................ 24

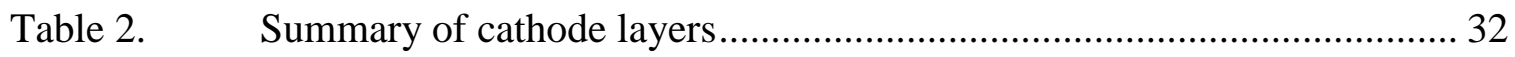

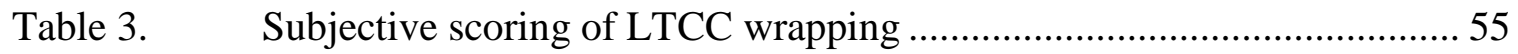

Table 4. Drying temperatures and times for silver pastes ................................ 61

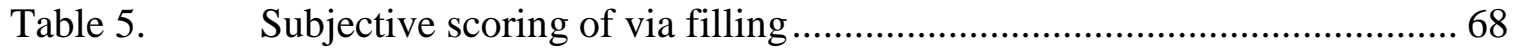

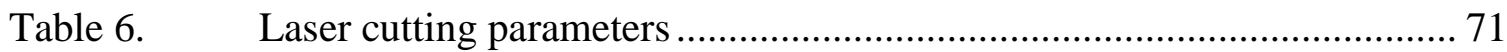

Table 7. Mill technique cutting parameters …............................................ 75

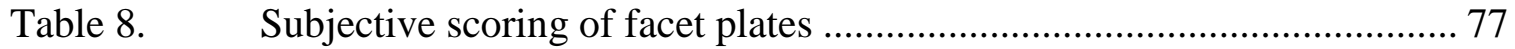

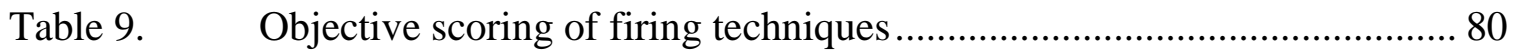

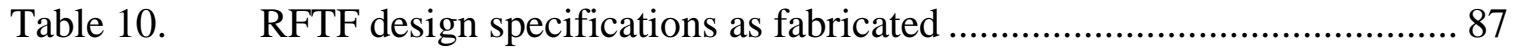

Table 11. Black and green printer settings................................................. 107 


\section{LIST OF FIGURES}

Figure 1. Gutton's Magnetron M-16 No.8 [1] ................................................... 5

Figure 2. (left) H. A. H. Boot and (right) J. T. Randall [1] ................................... 6

Figure 3. General Electric type E1189 [1] ..................................................... 7

Figure 4. (a) Magnetron lighting and (b) bulb diagram [26] ................................. 9

Figure 5. (a) (left) Comparing 1960's to (right) 2010's cooker magnetron [29] and

(b) microwave diagram [30] ...................................................... 10

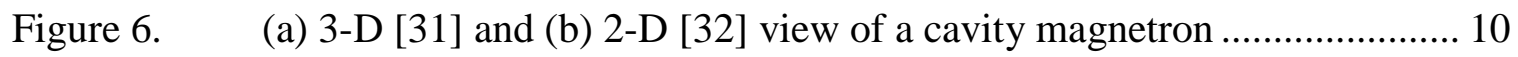

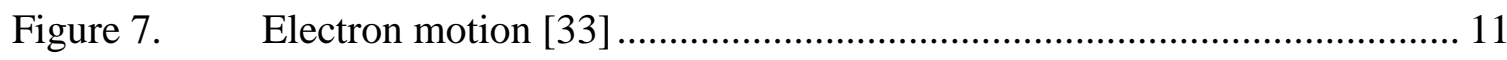

Figure 8. (a) Magnetron electron 'spokes' and (b-e) different cavity types [34] ..... 12

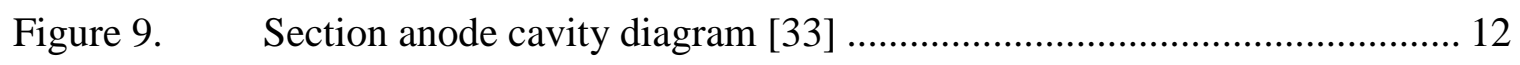

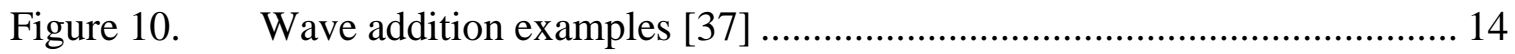

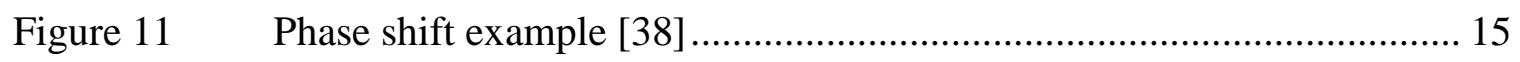

Figure 12. Relativistic magnetron expermental setup [5] ................................... 16

Figure 13. Self-injection phase locking experimental layout [6] ........................... 16

Figure 14. (a) Peer-to-peer locking experimental setup [7] and (b) actual [10]........ 17

Figure 15. (a) Gated vacuum field emitter diagram and (b) photograph of die [39].. 19

Figure 16. (a) Basic stripline [40] and (b) stripline with die diagram...................... 20

Figure 17. Cathode layers radially: (a) first ground plane, (b) dielectric with trace, (c) dielectric with second ground plane, (d) die support, and (e) facet plates 21

Figure 18. (a) A drawing of the cathode support stand which sits inside the vacuum test chamber, (b) drawing of the entire test chamber assembly showing the cathode support stand and faceted cathode inside the magnetron system 
surrounded by the anode circuit, (c) a close up drawing of the faceted cathode inside the magnetron circuit with an alignment piece to center the cathode inside the circuit, and (d) an image of the entire system showing the test chamber and the RF system components (electromagnet, dummy load, directional coupler, and coupling waveguide) courtesy of L3

Figure 19. (a) Cylindrical, (b) five sided, and (c) ten sided cathode [12] ................. 23

Figure 20. LTCC manufacturing process [48] ............................................... 25

Figure 21. Circuit diagram of the gated field emission cathode drive scheme with a pictorial representation of the gated emitter structure and hop funnels (not to scale). All of the power supplies are external to vacuum and a pulsed RF source drives the field emitter gate at the desired frequency. A high voltage power supply floats the cathode power supplies and cathode

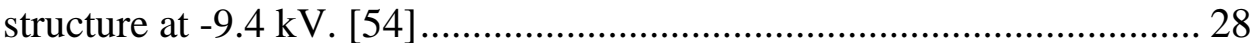

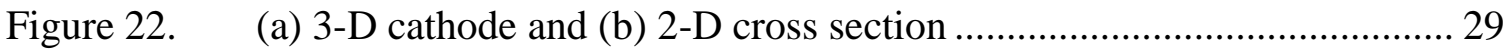

Figure 23. (a) LTCC layers 1, (b) 2, and (c) 3 outside and inside............................. 30

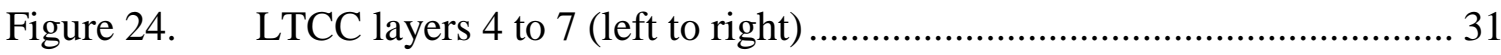

Figure 25. (left) Hop funnel primary and (right) secondary electron emission [55].. 31

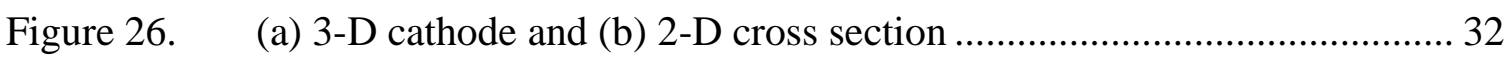

Figure 27. (a) IMS rolled structure [56] and (b) schematic [58] ............................ 34

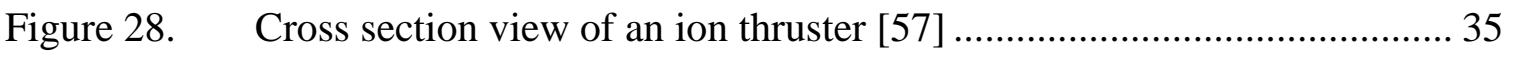

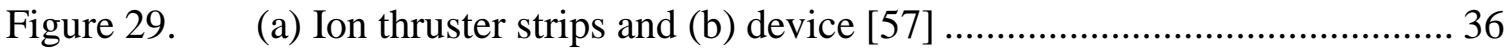

Figure 30. (a) $50 \mu \mathrm{m}$ punch, (b) $100 \mu \mathrm{m}$ via punched, and (c) drilled [63] .............. 37

Figure 31. (a) Different milling tools [71] and (b) end milling diagram [72].......... 40

Figure 32. (a) Snapshots of a laser pulse [70] and (b) how a laser cutter works [73] 41

Figure 33. (a) Building of a cap, (b) examples of bad, and (c) good circularity [57] 43

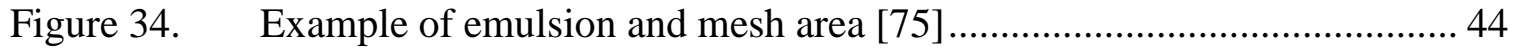

Figure 35. Example of screen printing [75] ................................................... 45 
Figure 36. (a) Quarter model rolled and (b) stacked cathode. 49

Figure 37. (a) Tightly wrapped and (b) loosely wrapped example cathode. 49

Figure 38. Layer by layer technique: layer 1 has been completely wrapped around the jig and layer 2 is about to be wrapped 51

Figure 39. Layer by group technique: layers 1 to 4 have been glued together with PEOX and are about to be wrapped around the jig..... 53

Figure 40. (a) Examples of not acceptable and (b) acceptable cathode wraps...........56

Figure 41. Cross section of the cathode structure showing both ground planes, the stripline, and one via clearance hole with a filled via.

Figure 42. (a) Circular via and (b) oval via 59

Figure 43. Flat fill technique: Stencil is placed on top of layer 3 which is placed on top of non-sticky mylar, then paste is squeegeed across the stencil ........ 60

Figure 44. Pneumatic pump technique: pressure is set, the nozzle is lined up with the vias on the cathode, then the button is pressed to push paste out of the nozzle

Figure 45. (a) Example of fully filled and (b) not fully filled vias 65

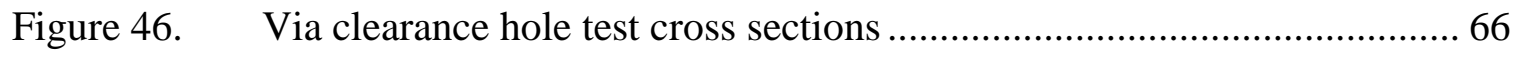

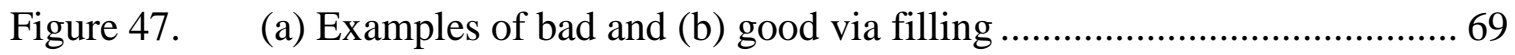

Figure 48. Diagram of facet plate (blue) fired geometry ................................... 70

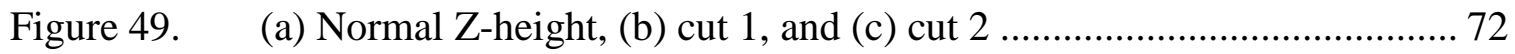

Figure 50. Laser technique: LTCC block is placed on the bed, then the laser burns the facet plate slots and outlines ........................................................ 73

Figure 51. Mill cutting cross sectional diagram ............................................... 75

Figure 52. Mill technique: LTCC block is taped to the bed, then the $60^{\circ}$ and $40^{\circ}$ endmills cut the slots and outlines of the facet plates, respectively......... 76

Figure 53. $\quad(a, c)$ Examples of laser and $(b, d)$ mill facet plates .................................. 78

Figure 54. Firing Techniques: (a) Horizontal, (b) caps, (c) stand, and (d) caps with stand 


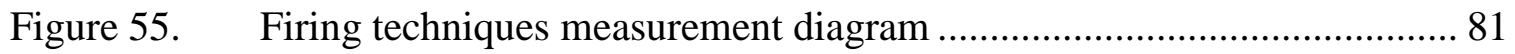

Figure 56. (a, c) Examples of bad and (b, d) good fired cathode structures .............. 82

Figure 57. (a) Fired cathode structure (b) with die and (c) with facet plates ............ 83

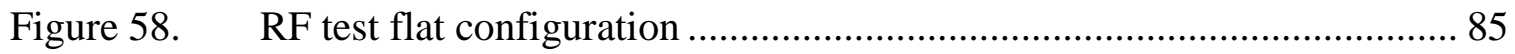

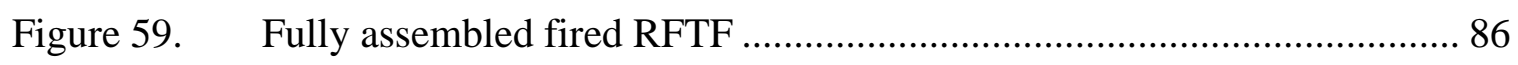

Figure 60. Cross section and components of a basic stripline diagram [79]............. 86

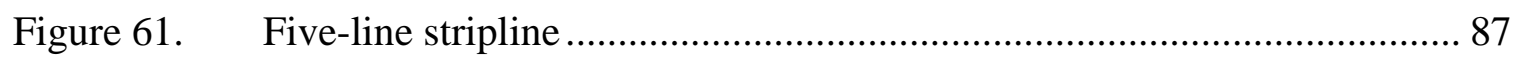

Figure 62. (a) Cutting straight lines on angled screen and (b) angled dots ............... 89

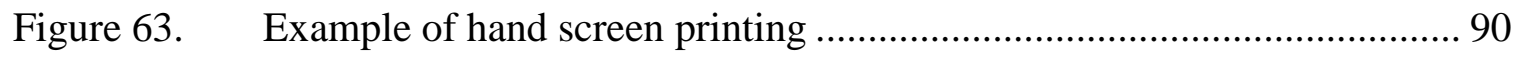

Figure 64. (a) Paste on the edge and (b) jumper layer paste diagram and (c) layers . 92

Figure 65. Position of LTCC on laser bed.................................................. 106

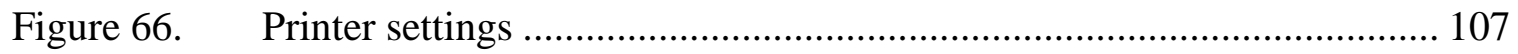

Figure 67. (a) Laser cutter menu Z-height and (b) file...................................... 108

Figure 68. Cut sheets of LTCC for one cathode.............................................. 108

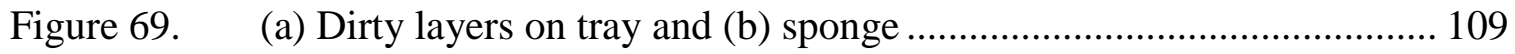

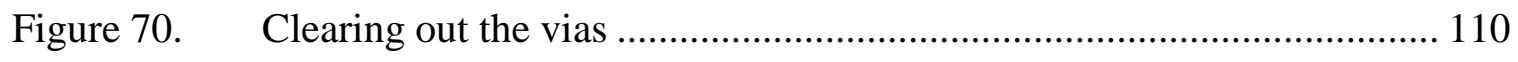

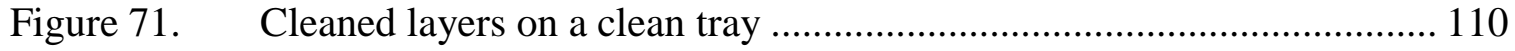

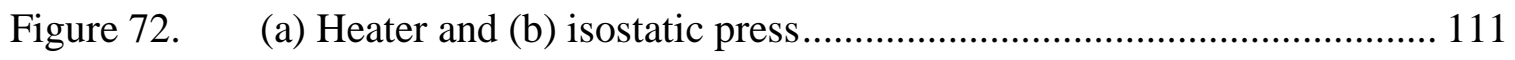

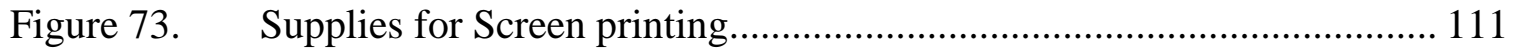

Figure 74. (a) Example layer 4 and (b) layers with paste.................................... 113

Figure 75. (a) Flat fill alignment, (b) squeegeeing, and (c) removal ..................... 114

Figure 76. (a) Drying with paper, (b) paper removal, and (c) drying again ............ 115

Figure 77. Top view of all seven completed layers........................................ 116 
Figure 78. (a) Bottom side and (b) top side of layer by group ............................ 117

Figure 79. (a) Jig preparation, (b) jig wrapping, and (c) rolled layer 1 to $4 \ldots \ldots \ldots \ldots . . . .118$

Figure 80. (a) Lay flat, (b) align layer 5, (c) flip over, (d) roll, (e) final................. 119

Figure 81. (a) Laminated, (b) layer 6, (c, d) spacer strips, (e) fully wrapped cathode

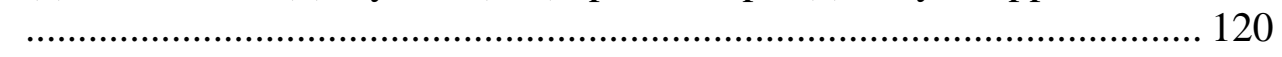

Figure 82. (a, b) Vacuum bag process and $(\mathrm{c}, \mathrm{d})$ isostatic press ........................... 121

Figure 83. (a, c) Before compared to $(b, d)$ after lamination .................................. 121

Figure 84. (a) Removing tabs, (b) cathode, (c) wax paper, and (d) placing on stand 122

Figure 85. LTCC system firing temperature profile......................................... 123

Figure 86. (a) Rectangular sheets, (b) lamination, and (c) fixturing to mill ............ 123

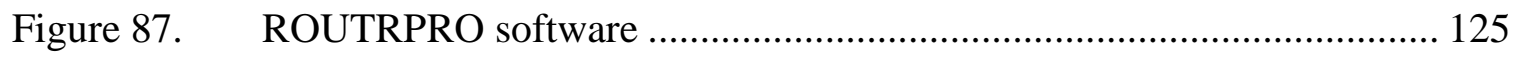

Figure 88. (a) Green facet plate and (b) fired slits showing verifications ................ 126 


\section{CHAPTER ONE: INTRODUCTION}

\section{Motivation}

Magnetrons are microwave oscillators that are used extensively for commercial and military applications. They were produced in the early 1920's and helped lead the allies to victory in WWII with the implementation in radar systems [1]. Today, almost every household and even dorm room has one to rapidly cook food. The microwave oven has a magnetron that produces a $\sim 6 \mathrm{~cm}$ (2.4 in) wavelength wave that rapidly vibrates the water molecules in the food, heating up and cooking the food. Most magnetrons today produce a wavelength of about $1 \mathrm{~m}$ to $1 \mathrm{~mm}$, depending on the application.

Because of important applications such as space solar power stations and highpower transmitters, there has been an increased demand for greater power capacities of magnetrons. There is a major concern that higher levels may be unattainable because of the inherent limits on the electric field sustainable in resonant cavities [2]. The solution is to phase lock multiple low power (inexpensive) magnetrons together to achieve the same power as one, high power (expensive) magnetron. Traditional phase locking uses external or additional devices that are attached to the magnetron $[3,4,5,6,7,8,9,10]$. These devices, regulate, measure, and change the inputs into the magnetron to allow for accurate phase control. The new proposed method is to alter the magnetron components themselves to introduce an internal phase locking ability.

The Vacuum Electron Devices (VED) and Ceramic Micro Electrical Mechanical Systems (CMEMS) laboratories at Boise State University have designed a new cathode 
that uses gated vacuum field emitters that will precisely inject electrons into the system. The emitters will be modulated by a radio frequency embedded transmission line (stripline). This controllable electron injection has been shown in simulation to decrease startup times, increase efficiency, and even allow for active phase control during oscillation $[11,12,13,14,15,16]$.

\section{Contributions}

Throughout this research, the manufacturing process of the field emitter cathode structure was developed and optimized. In addition to the cathode structure, new low temperature co-fired ceramic (LTCC) manufacturing techniques were developed in order to simplify the build process. These techniques include LTCC rolling/wrapping, via filling, machining, and firing techniques. In addition to these, the screen printed embedded stripline for the cathode was prototyped. The following are contributions to the cathode project:

1. Two different rolling/wrapping techniques were developed: layer by layer which wraps one layer at a time and layer by group which wraps multiple layers at a time.

2. Two different via filling techniques were developed: flat fill which fills each layer of the via at a time and the pneumatic pump fill which fills the whole via at a time on the already rolled structure.

3. Two different machining techniques were developed: one using a laser and another using a mill to cut ' $\mathrm{V}$ ' shaped slots. 
4. Two different firing techniques were developed: stand and stand with caps; however, four techniques were compared to maintain circularity and linearity of the cathode while firing.

5. The embedded stripline was developed and different thin screen cutting/printing processes were used for rapid prototyping.

\section{Overview of Thesis}

This thesis is broken down into five chapters that give detailed descriptions of the research conducted.

- Chapter 2 presents background information on history, applications, components, and traditional phase locking of a magnetron. It also discusses the material, modeling, and design of the new cathode. Lastly, it discusses some of the previous techniques for fabricating LTCC structures including wrapping, via filling, machining, firing control, and screen cutting/printing.

- Chapter 3 presents the LTCC rolling/wrapping, via filling, machining, and firing techniques that were compared.

- Chapter 4 presents the radio frequency test flat (RFTF) and thin screen cutting/printing techniques used for rapid prototyping.

- Chapter 5 presents conclusions, results, and future work for the cathode project. 


\section{CHAPTER TWO: BACKGROUND}

This chapter will discuss background information on history, applications, components, and traditional phase locking of a magnetron. It also discusses the material, modeling, and the design of the new cathode. Finally, the current LTCC manufacturing techniques will be summarized for their strengths and weaknesses.

\section{Magnetron}

\section{$\underline{\text { History }}$}

The history of the magnetron dates back to the second decade of the 1900's. In 1917, Albert W. Hull, at General Electric (GE), proposed to use a magnetic field instead of an electric field to control the electric current in a valve. This was an attempt to circumvent the famous Lee de Forest's triode patent [1]. GE found that it would be easier to buy the patent and thus led to the term "magnetron" which was coined in a 1921 paper [17].

Most of the oscillators at the time had wavelengths just shy of several hundred meters. It wasn't until 1920 that Heinrich Barkhausen reached a $30 \mathrm{~cm}$ wavelength (1 GHz) limit with his Barkhausen oscillator [1]. In 1928, Kinjiro Okabe of Japan, reached a wavelength of $5.6 \mathrm{~cm}$ under Professor Hidetsugu Yagi (a former student of Barkhausen). This split anode [18] magnetron created a huge stir in Europe which lead to significant improvements.

In 1930, Maurice Ponte, from the French company SFR-CSF, adopted Okabe’s principals with a goal of giving the magnetron field applications. Ponte's first trials were 
$40 \mathrm{~W}$ at $3 \mathrm{~m}$ wavelength $(100 \mathrm{MHz})$ with a $20 \mathrm{~mm}$ split anode diameter and then $80 \mathrm{~cm}$ wavelength $(375 \mathrm{MHz})$ with a $5 \mathrm{~mm}$ anode diameter [1]. Henri Gutton, who succeeded Ponte in 1934, worked for years developing the multi segment anode magnetron. In 1938, Gutton developed an eight-segment anode magnetron shown in Figure 1c. Figure 1a and Figure $1 \mathrm{~b}$ shows the drawing and prototype, respectively, of the cathode and anode. This magnetron was capable of reaching $10 \mathrm{~W}$ at a $16 \mathrm{~cm}$ wavelength, with a $15 \%$ efficiency, under $765 \mathrm{~V}$ and 430 gauss for the anode voltage and magnetic field, respectively [1].

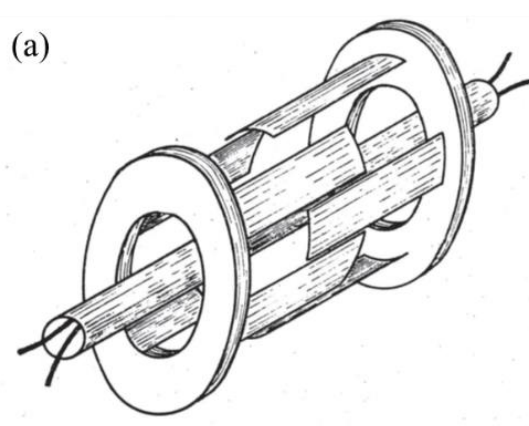

Figure 1.

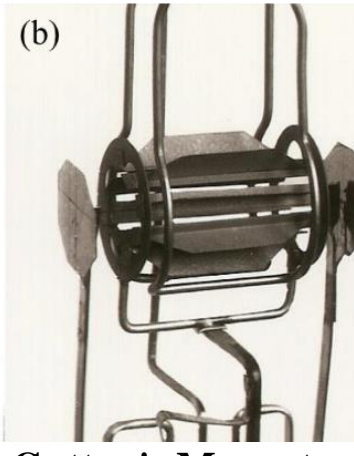

Gutton's Magnetron M-16 No.8 [1] (c)

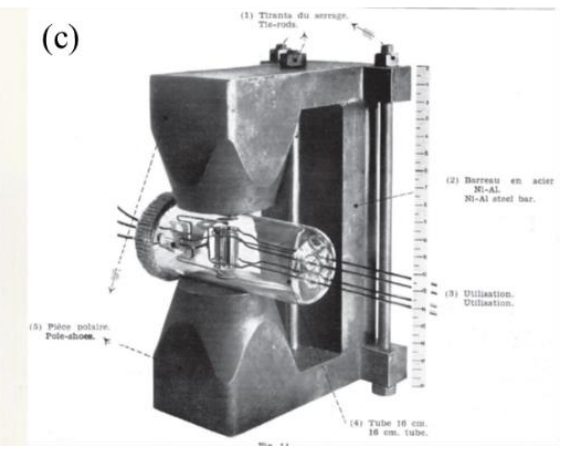

In 1937, Australian Professor Marcus L. E. Oiliphant came to Birmingham

University where he worked on high-power radar under the British Admiralty. From 1938 to 1940 , there was a substantial need to improve the airborne and surface radar systems. One of the improvements was to make a cavity magnetron that could produce at least $1 \mathrm{~kW}$ of power at a wavelength of $10 \mathrm{~cm}$ [1]. Radar systems work by sending out an electromagnetic wave of radiation through an antenna which reflects off of an object (airplane, boat, etc.). These reflections are analyzed to produce a detailed map (radar map) of the surroundings. At the time, this technology was highly sought after and would ultimately help the allies win WWII. 
On February 21, 1940, two researchers from Oiliphant's lab, John T. Randall [19] and Henry A. H. Boot, shown in Figure 2, developed a magnetron that achieved $400 \mathrm{~W}$ at a wavelength of $9.8 \mathrm{~cm}$ in a vacuum. A few weeks later, Randall and Boot succeeded when they produced power levels in the kilowatts. In September, they developed a 14cavity magnetron operating at $5 \mathrm{~cm}$ and another 6-cavity magnetron operating at $3 \mathrm{~cm}$. Finally, in May 1940, they produced a 1 MW magnetron with a wavelength of $10 \mathrm{~cm}$ [1].

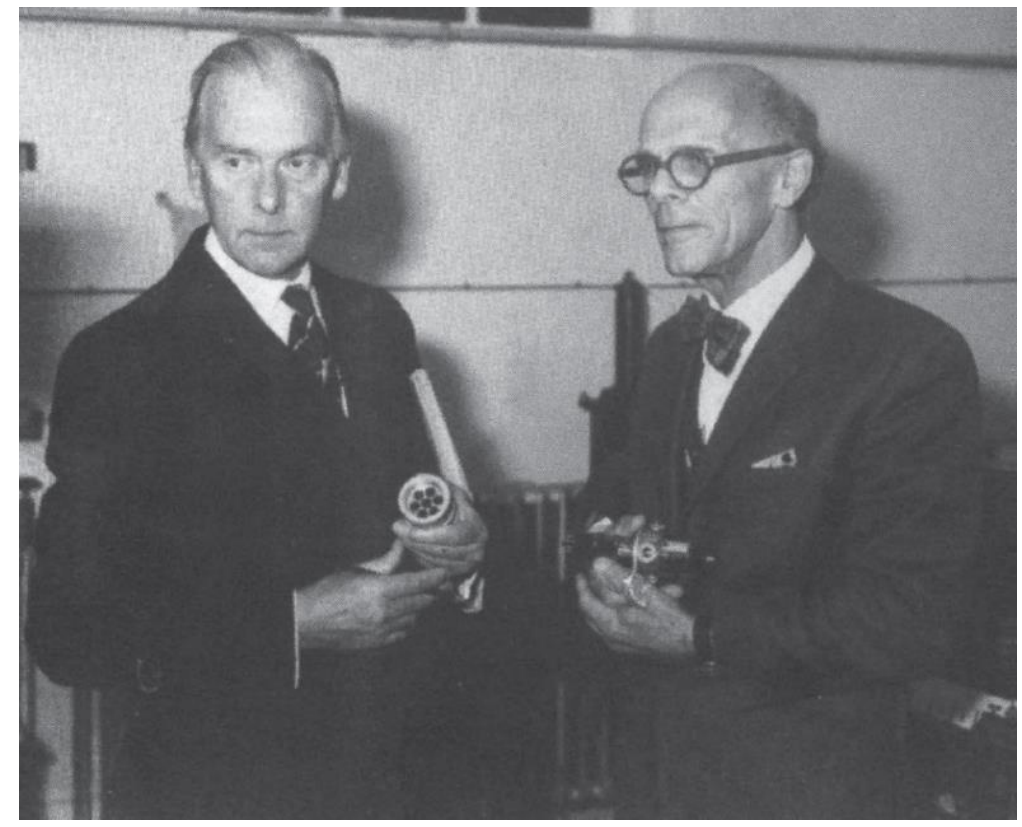

Figure 2. $\quad$ (left) H. A. H. Boot and (right) J. T. Randall [1]

The magnetron previously discussed however, was only a laboratory prototype. The next step was to produce a field operable system which General Electric Company Ltd. (GEC) at Wembley did in August 1940. GEC produced a radar consisting of a $10 \mathrm{~cm}$ magnetron and two small paraboloids as transmitting and receiving antennas that detected the first microwave echoes of an aircraft [20, 21]. Eric C. Stanley Megaw, a team lead at GEC, modified the Boot and Randall design by coating the center cathode in oxides which lead to a $100 \mathrm{~kW}, 10 \mathrm{~cm}$ wavelength magnetron. 
Amidst Word War II, the United Kingdom (UK) realized that the German's industrial power was going to outperform their own. The scientific advisor to the British government, Sir Henry T. Tizard, suggested disclosing their technical information to the United States, so that their (U.S.) enormous potential for development and manufacturing could be put into effect. On August 9, 1940, Winston Churchill approved the "Tizard mission," which gave the go ahead to send the cavity magnetron developed by Megaw to the United States [22, 23]. In early September, E. G. Bowen brought the type E1189, series N. 12, shown in Figure 3, to North America which was capable of producing 10 $\mathrm{kW}$ at $10 \mathrm{~cm}$.

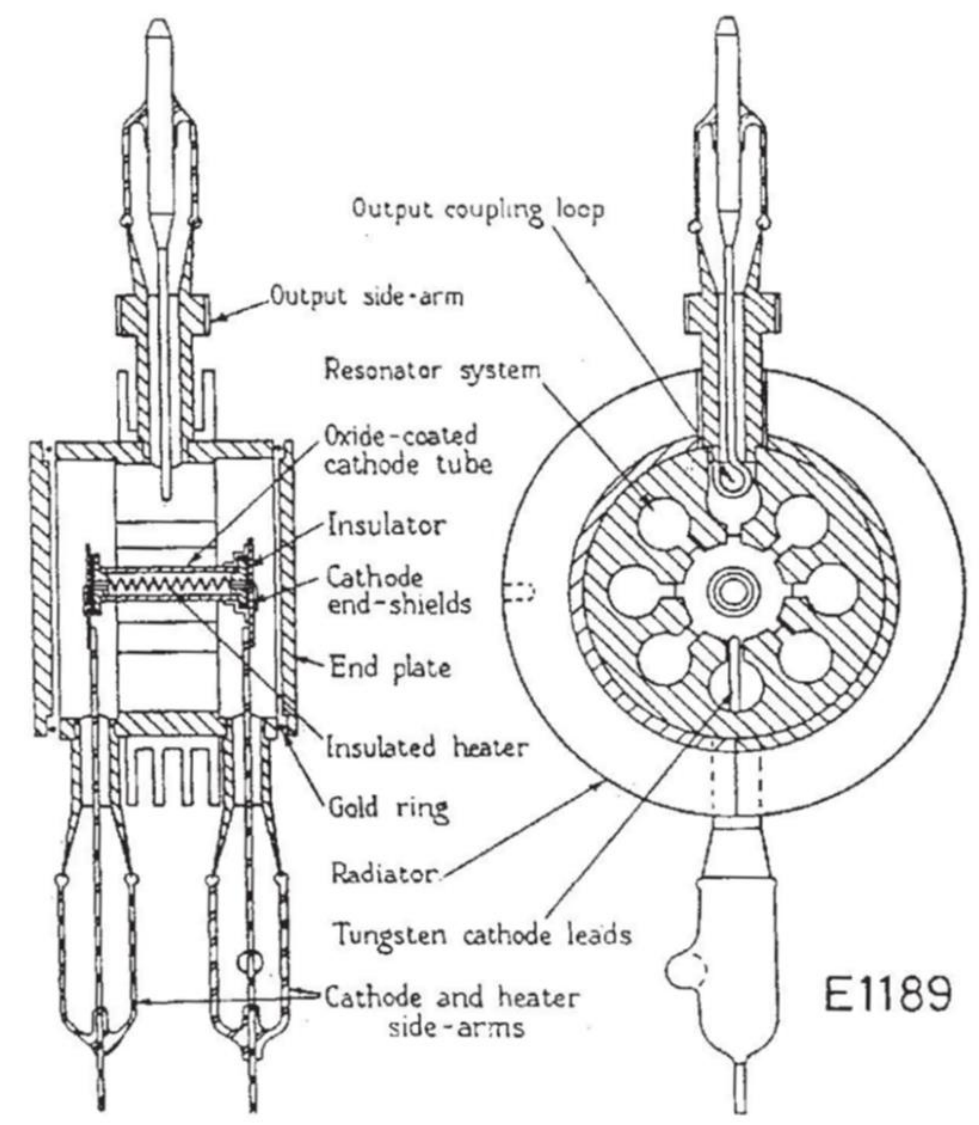

Figure 3. General Electric type E1189 [1] 
The cavity magnetron was quickly mastered by the U.S. and in the fall of 1940, the Massachusetts Institute of Technology (MIT) created the Radiation Laboratory. This laboratory provided microwave radars to all the allied forces, giving them a technological advantage. Even though Boot and Randall are credited as inventors of the cavity magnetron [24], in actuality, it was a world-wide effort that spanned almost three decades.

$\underline{\text { Applications }}$

Besides radar as discussed above, other applications of the magnetron include magnetron lighting and microwave ovens. Magnetron lighting was an attempt at developing a mercury-free, low infer-red, and electrodeless lighting source. In 1990, Fusion System Corporation succeeded when they developed a benign sulfur filled lamp optimized for visible light [25]. Traditional high-intensity discharge (HID) lamps produce visible light with two metal electrodes that arc, heating the gas or plasma inside. These electrodes can be a significant deleterious factor for bulb life [26].

The sulfur bulb's plasma is heated externally by microwave energy, eliminating the need for electrodes altogether. These sulfur lamps contain non-reactive fill materials and are electrodeless which leads to virtually no shift in spectrum over their lifetime. These lamps, in 1994, where tested to 10,000 hours with no evident failure to the bulb, which suggested life expectancy to depend only on magnetron durability [26]. The microwave lighting diagram and sulfur bulb diagram are shown in Figure 4a and Figure $4 \mathrm{~b}$ respectively. 


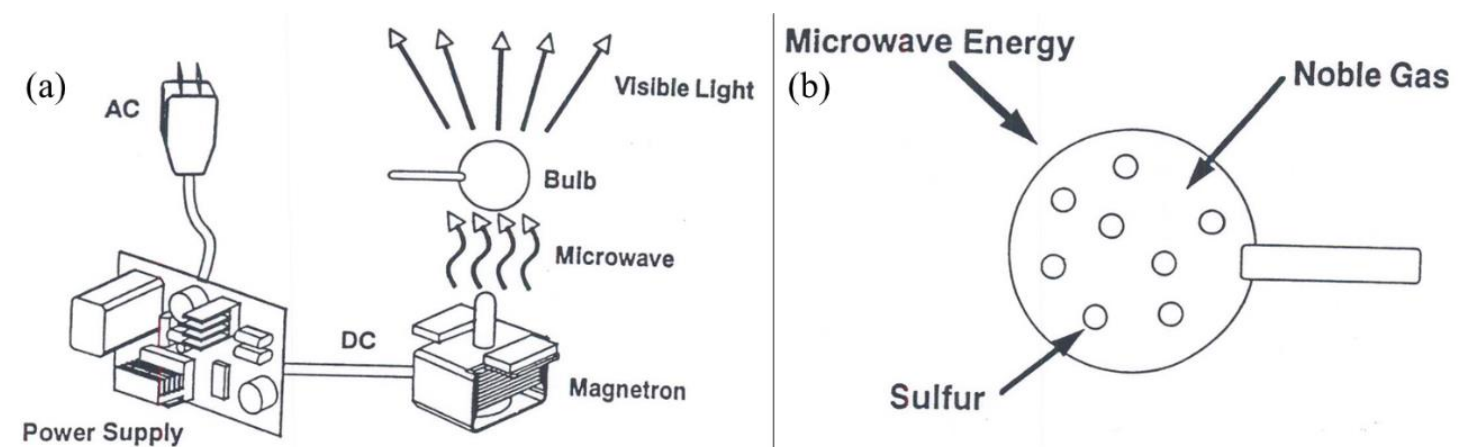

Figure 4. (a) Magnetron lighting and (b) bulb diagram [26]

The microwave oven was invented shortly after WWII by the company Raytheon. During the war, Raytheon had been producing magnetrons for radar systems at a rate of 17 per day [27]. Raytheon researcher Percy Lebaron Spencer discovered the novelty of microwave cooking and filed the patent in 1950 [28]. By 1947, the first commercial microwaves were produced which stood nearly six feet tall and weighed over 700 pounds [27]. By 1970, microwaves had started to resemble the ones seen today. Microwaves cook food by funneling the $6 \mathrm{~cm}$ radio waves from a magnetron through a wave guide to the food compartment. The waves travel through the food, causing the water molecules to vibrate rapidly and heat up, which cooks the food. Figure 5a show a size comparison between the Raytheon QK707 (1960's) and the modern (2010's) cooker magnetron. Figure $5 \mathrm{~b}$ shows a diagram of the magnetron, wave guide, and radio waves in a microwave. 

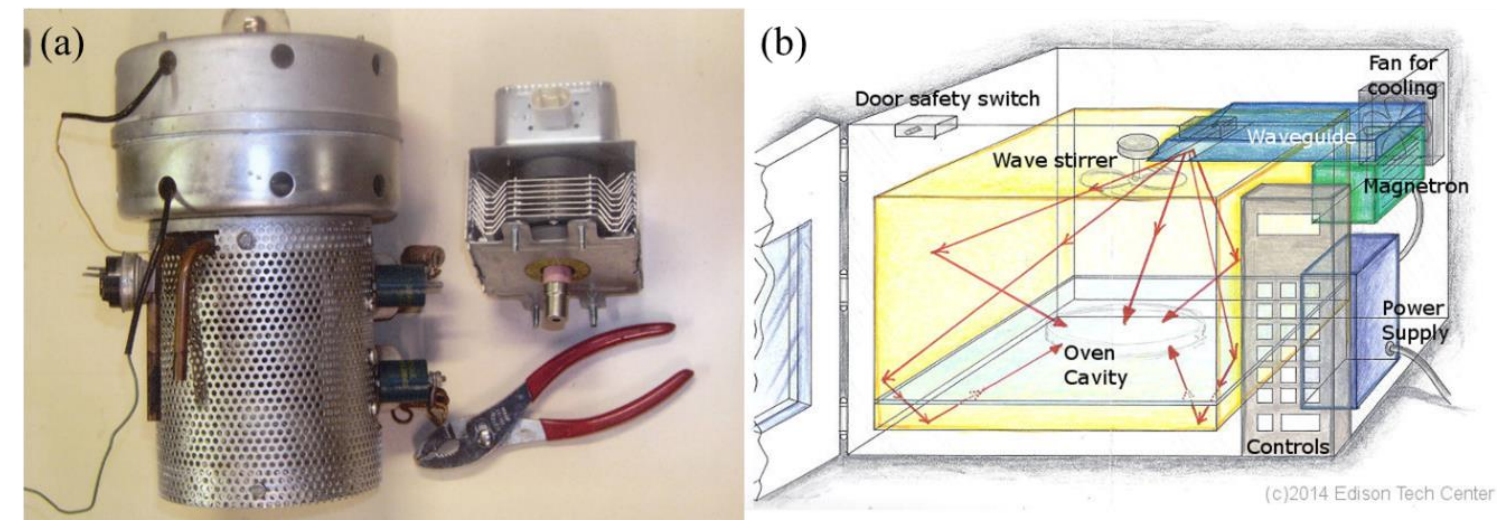

Figure 5.

(a) (left) Comparing 1960's to (right) 2010's cooker magnetron [29] and (b) microwave diagram [30]

\section{Components}

A cavity magnetron is composed of four main components: a cathode, anode, two magnets, and an antenna. A three-dimensional view and a two-dimensional view of a magnetron are shown in Figure 6a and Figure 6b respectively.
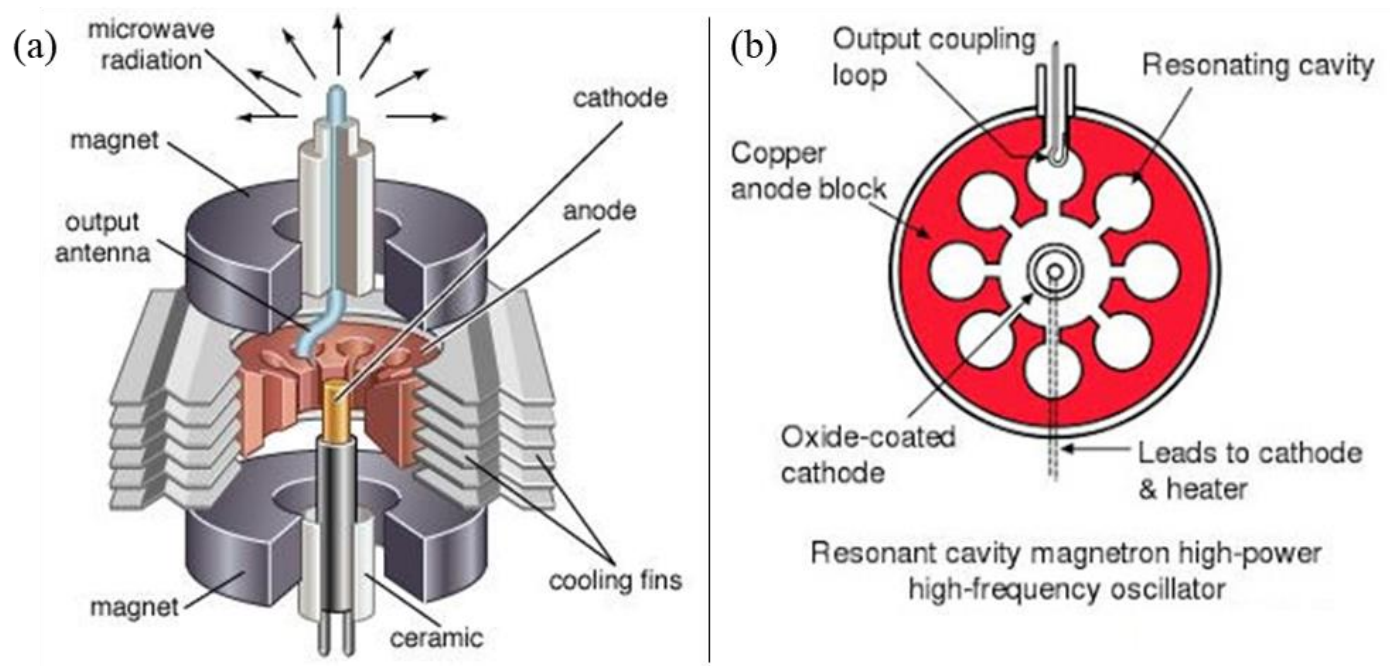

Resonant cavity magnetron high-power high-frequency oscillator

\section{Figure 6. (a) 3-D [31] and (b) 2-D [32] view of a cavity magnetron}

The magnetron typically uses thermionic emission, which is the process of heating up the center, negatively charged cathode, until electrons are generated. These electrons have a naturally tendency, due to the electric field, to emit radially outward towards the positively charged anode. The magnets on both ends, however, create a 
magnetic field that creates a Lorentz force. This force causes the electrons to spiral around (azimuthally) the cathode, as opposed to outward (radially). This is shown in Figure 7.

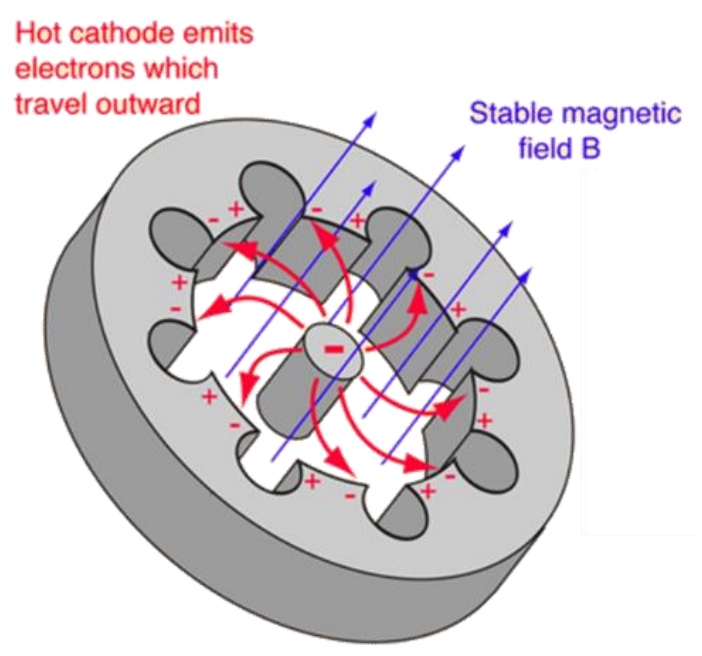

Figure 7. Electron motion [33]

At this point, the electrons have not reached the anode yet and are close together (forming an electron cloud), spiraling around the cathode. An instability in the electron cloud will result in feedback with the resonant cavity circuit. This feedback causes the cloud of electrons to form spokes as shown in Figure 8a. Over time ( 100 nanoseconds), this process reaches steady state and the tips of the spokes finally reach out and sweep past the anode cavities, touching the anode. Different types of resonant cavities are shown: slot (Figure 8b), vane (Figure 8c), rising sun (Figure 8d), and hole and slot (Figure 8e). 


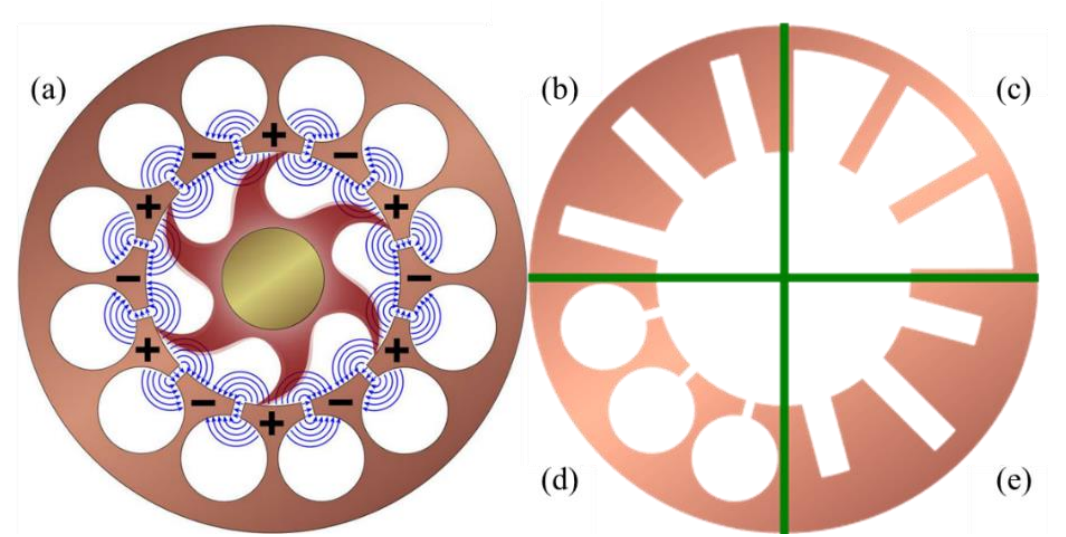

Figure 8. (a) Magnetron electron 'spokes' and (b-e) different cavity types [34]

The electrons sweeping past the anode cavities changes the polarity of both ends of the cavity, shown in Figure 9. The spoke rotation results in time-varying electric and magnetic fields - electromagnetic waves. The waves are then funneled through an antenna, or coupling point, located in one of the resonant cavities, shown back in Figure 6a. The antenna sends the waves through a waveguide where they are guided to the desired location.

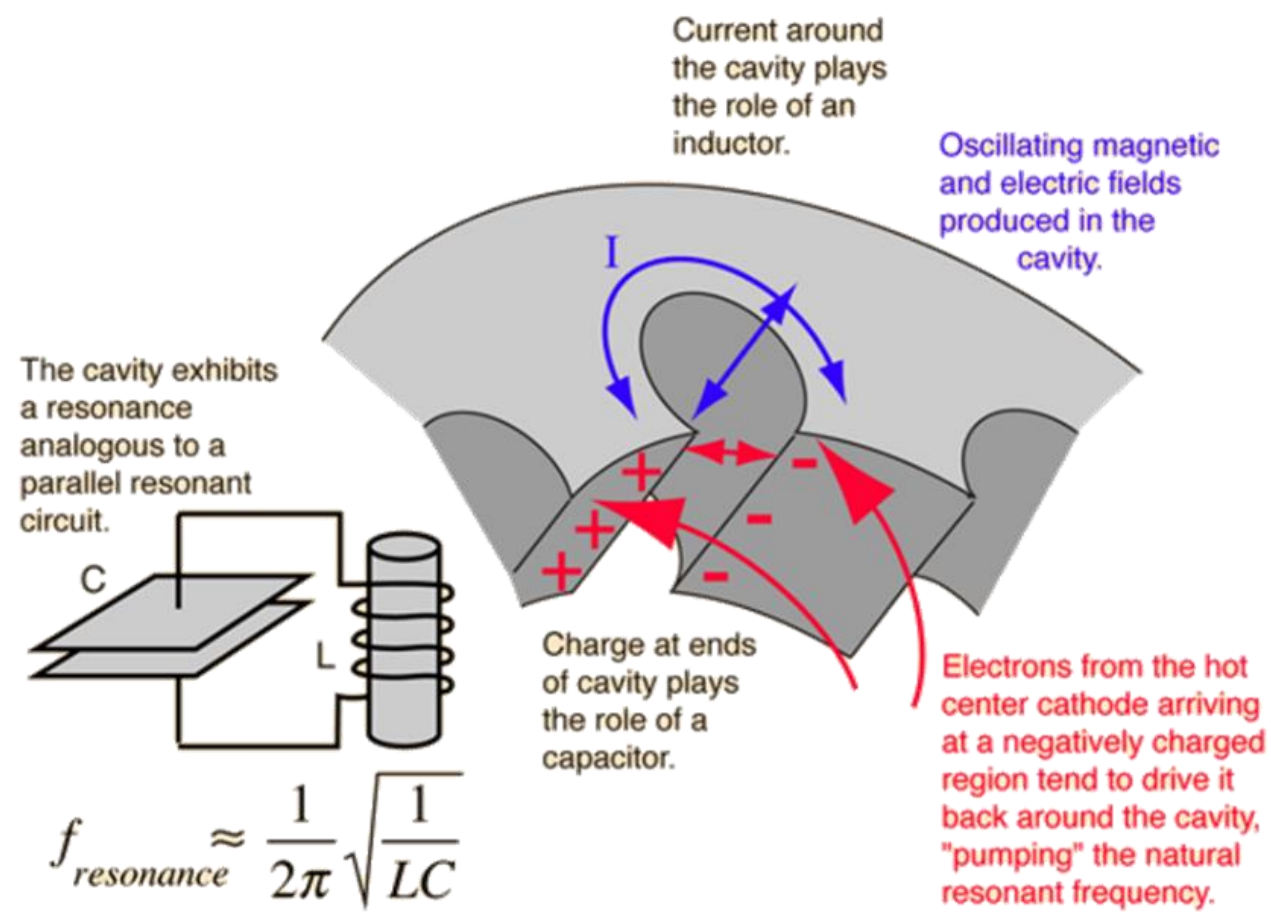

Figure 9. Section anode cavity diagram [33] 


\section{$\underline{\text { Phase-Locking }}$}

Magnetrons are widely used for numerous applications because of their high efficiency and low cost. Because of important applications such as space solar power stations, electronic warfare, and high-power transmitters, there has been an increased

demand for greater power capacities [2]. There is a major concern that higher levels may be unattainable because of the inherent limits on the electric field sustainable in resonant cavities. In addition, the power density limit set by air breakdown argues for distribution of power into an array of radiating antennas [35]. One such solution is to combine an array of magnetrons together in phase, also known as phase-locking. The concept is that by adding the power of multiple inexpensive magnetrons together (same frequency and wavelength), one could produce the same output power as a single expensive magnetron. Interference describes the interaction of two waves with each other and the resulting formation of a new wave. The relevant parameter for the interference of light waves is the amplitude, which is the highest point of the wave. If two waves interfere, the amplitude of the resulting wave will be equal to the vector sum of the amplitudes of the two interfering waves [36]. Simply put, if two of the same waves are added together, the resulting wave will be twice the amplitude. An example of wave addition for two and three of the same phase waves, is shown in Figure 10a. Figure 10b shows how two waves, phase shifted by $\pi / 2$ and added together, do not result in twice the amplitude. 

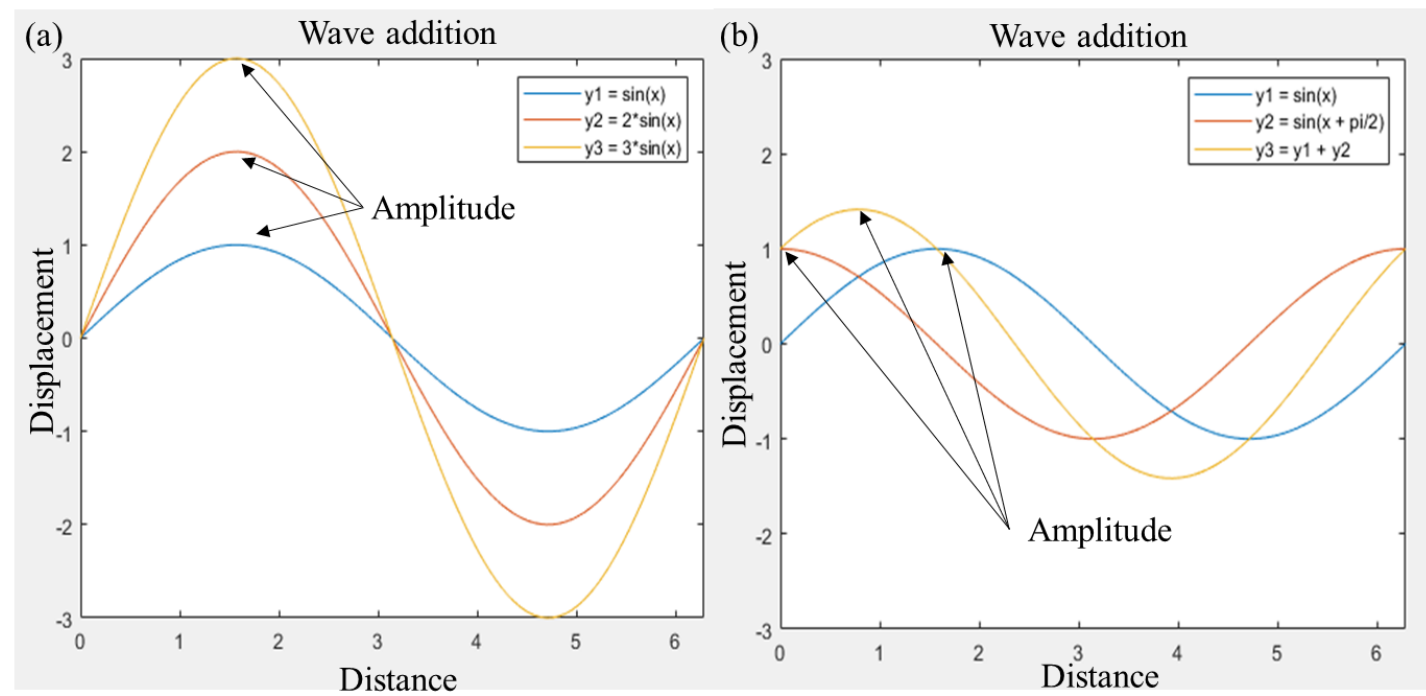

Figure 10. Wave addition examples [37]

The most important component of wave addition is the phase angle of the waves.

If the phase of each wave is not the same, then the waves can be deconstructive or subtract from each other. An example of two waves phase shifted by $\theta$ (theta) is shown in Figure 11. These waves will not produce as much peak power, as if they were directly on top of each other, also known as in-phase $(\theta=0)$. By controlling the phase, the two waves produced by two different magnetrons could be locked together, $(\theta=0)$ producing a phase locked wave with twice the power. If phase locked correctly, an $\mathrm{N}$ number of magnetrons could produce a wave of $\mathrm{N}$ times the power of each magnetron. 


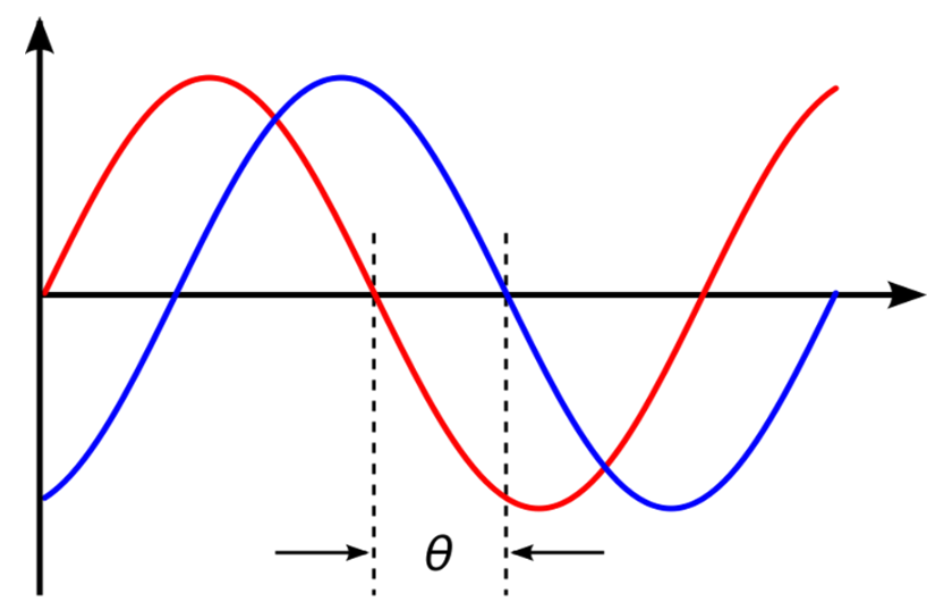

Figure $11 \quad$ Phase shift example [38]

The study of phase-controlled magnetrons has produced numerous methodologies, mostly branching from Robert Adler's research [3]. Some methods include external injection-locking, self-injection-locking, and peer-to-peer locking. External injection locking, also known as the master slave method, is where the frequency and phase of a 'slave' magnetron can be controlled by an injected microwave pulse from a second 'master' magnetron [4]. Another form of external injection locking, is when both magnetrons are fed from a split magnetically insulated transmission line. A phase discriminator measures the phase and power of both magnetrons and adjusts the coupling bridge to bring both magnetrons into phase [5], shown in Figure 12. 


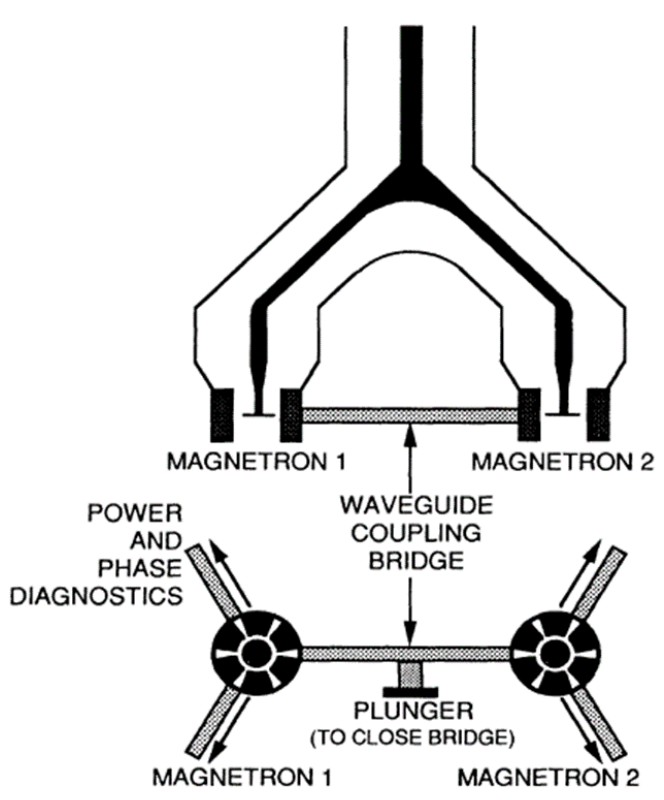

Figure 12. Relativistic magnetron expermental setup [5]

Self-injection locking has been used to reduce noise in lasers and to provide frequency locking and noise reduction in magnetrons. This is achieved by placing a frequency-tunable high-quality factor $(\mathrm{Q})$ dielectric resonator (DR) in a feedback loop, shown in Figure 13. The locking frequency varies by adjusting the resonant frequency of the high-Q cavity which shows significant noise reduction near the phase locked frequency [6].

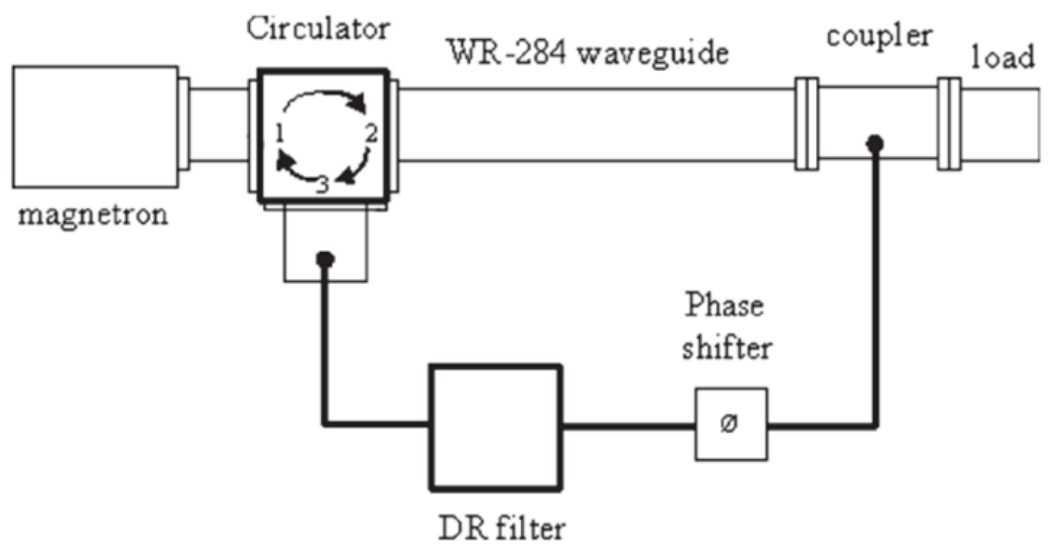

Figure 13. Self-injection phase locking experimental layout [6] 
Peer-to-peer locking is an attractive alternative to injection locking when a large number of magnetrons are involved, because it employs a lower number of expensive components such as isolators and circulators [7]. The current into magnetron 2 is due to the combined effects of magnetron 1 and the electromagnetic coupling between magnetron 1 and 2 [8], and vice versa for the current into magnetron 1, shown in Figure 14a. In 1991, the Physics International Company, successfully connected seven magnetrons in peer-to-peer phase lock and produced 2.9 GW [9], shown in Figure 14b.

(a)

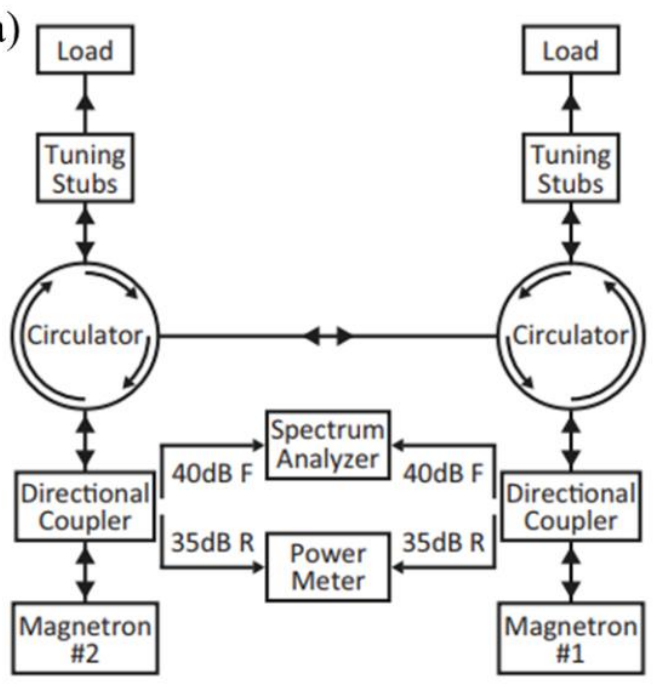

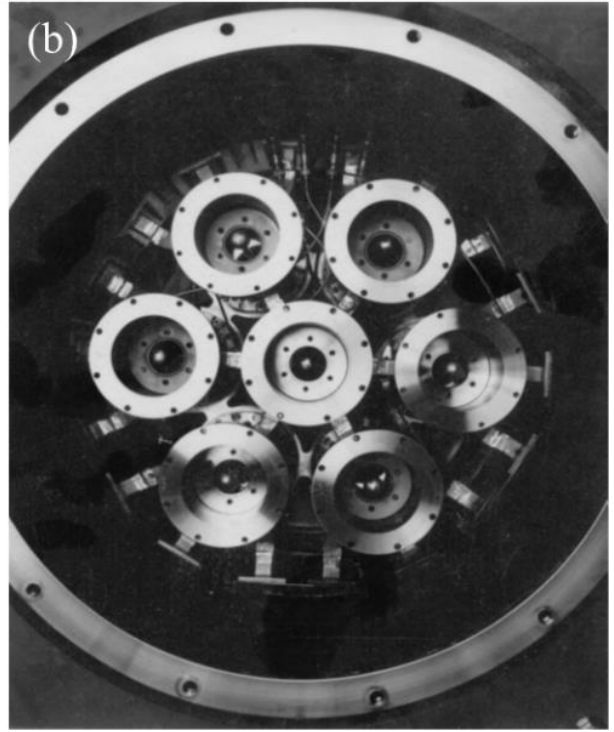

Figure 14. (a) Peer-to-peer locking experimental setup [7] and (b) actual [10]

As previously stated, the magnetron has numerous applications varying from military radar, all the way to at home rapid cooking. The magnetron is a simple and robust device that can produce power at varying wavelengths. It has also gone through years of development to increase efficiency as well as power producing capabilities. In order to increase power production further, multiple magnetrons can be phase locked together which was demonstrated by methods such as peer-to peer locking or injection locking. 


\section{Cathode}

Traditional magnetron cathodes use thermionic emission. This process, while robust and widely used, offers no control of emission as well as allows for ion back bombardment which can damage the cathode and keep it in a heated thermionically emitting state [11]. In addition to the emission process, the current techniques for phase locking uses external sources to control and lock the phase of the magnetrons. These methods, while effective, require additional devices to control the phase of the magnetron which can be bulky and expensive.

The proposed new method to combat these challenges is through a gated field emission cathode [11]. This cathode is composed of arrays of gated vacuum field emitters (die) that will emit electrons at specific locations and times rather than continuously (thermionic emission). These emitters are protected from ion back bombardment by facet plates that are attached to the surface of the cathode. This new design modifies the already existing cathode to allow for active phase control. Figure 15 shows the gated vacuum field emitters (die) proposed for this research. Each die has two field emission areas (elements). 


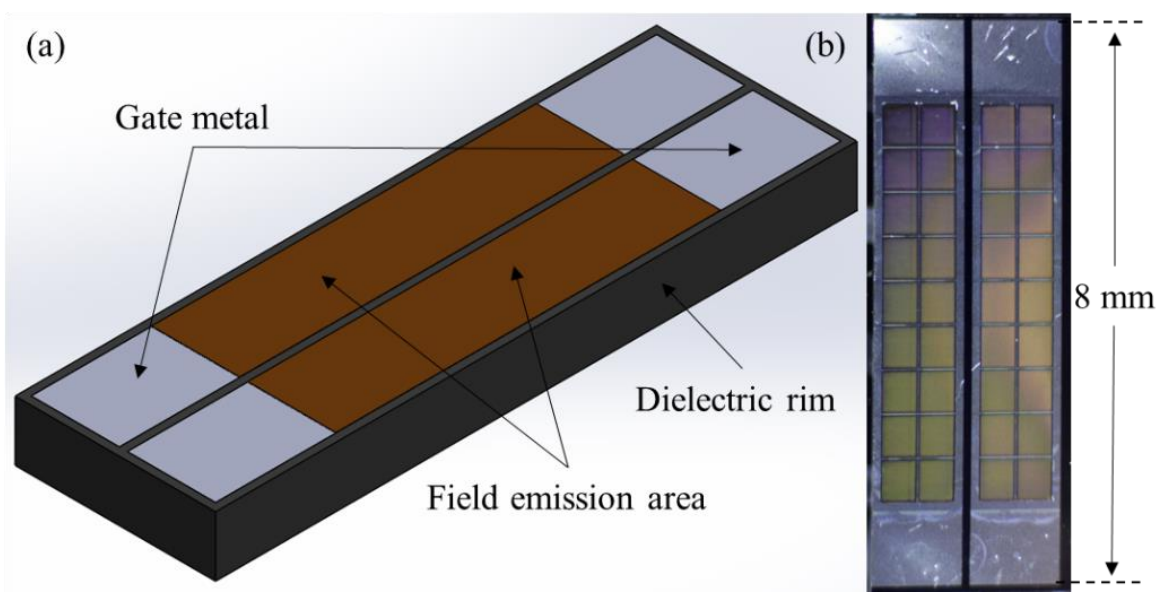

Figure 15. (a) Gated vacuum field emitter diagram and (b) photograph of die [39]

The die will be activated by a stripline, which is a transmission line that is sandwiched between two ground planes. Figure 16a shows a diagram of a basic stripline which is composed of a ground plane, dielectric layer, transmission line (trace), another dielectric layer, and another ground plane. The physical characteristics of the stripline $(\mathrm{H}$, $\mathrm{T}$, and $\mathrm{W}$ dimensions) as well as material properties directly relate to the electrical properties which will be discussed later in Chapter 4. Figure 16b shows a diagram of the stripline with a die that will be used for the cathode structure. The differences are that the die rests on top of the second ground plane and a via is used to complete the transmission line to die connection (tdc). This via will have to pass through a hole in the top ground plane (via clearance hole) that must be large enough to prevent shorting between the ground plane and the via. The via to die connection shown in Figure $16 \mathrm{~b}$ is not actually yellow but was colored yellow for clarity. This is used to connect from the via to the bond pad on the die. Since each die has two emission areas, each die will require two vias, one on each side as shown in Figure 16b. For the cathode structure, these vias will be above and below each die. 
(a)

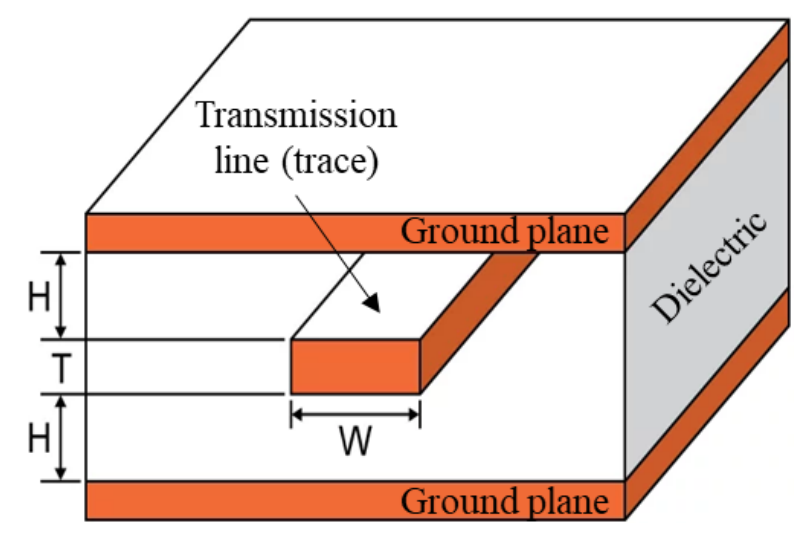

(b)

Via to connect other side of die to trace

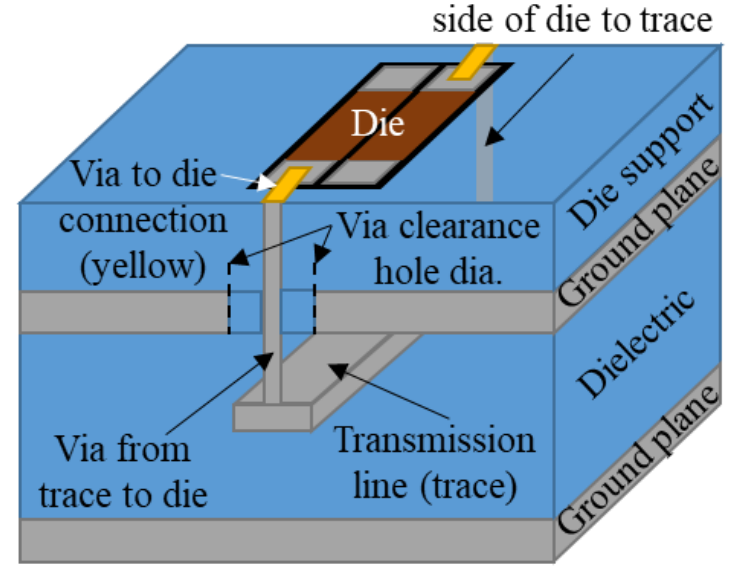

Figure 16. (a) Basic stripline [40] and (b) stripline with die diagram

The new cathode that replaces the old thermionic emission cathode as previously mentioned will have die on every side of the tube structure with facet plates that protect the die. This cathode structure will be composed of the cross sections shown previously in Figure 16b that spiral around the tube. The new cathode structure will consist of 7 layers including (working outward radially): an internal first ground plane (L1, Figure 17a), a dielectric layer with a trace (L2, Figure 17b), another dielectric layer with a second ground plane (L3\&4, Figure 17c), die support layers with die slots (L5-7, Figure 17d), and finally facet plates with die placed in the slots (Figure 17e) to complete the full cathode assembly. In order to connect to all of the vias, the trace layer will need to spiral around the cathode structure in a barber pole style as shown in (Figure 17b). A more detailed and labeled diagram will be shown later in the Design section. 
(a)

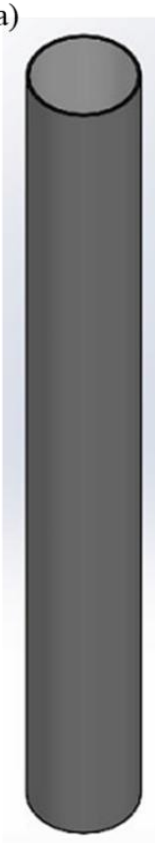

(b)

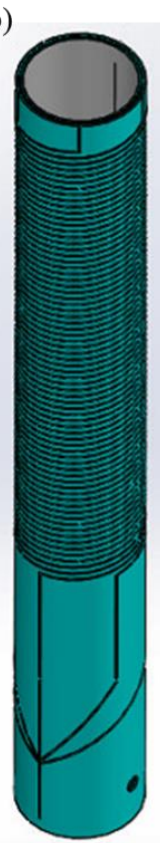

(c)

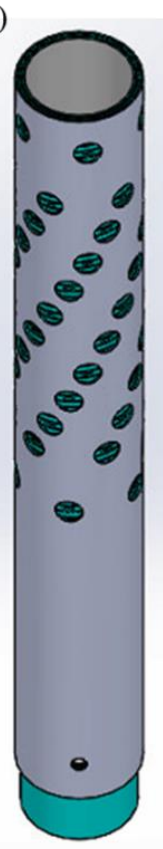

(d)

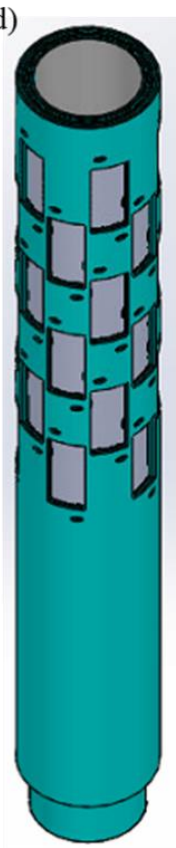

(e)

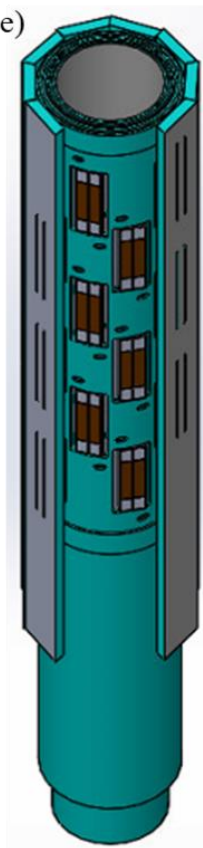

Figure 17. Cathode layers radially: (a) first ground plane, (b) dielectric with trace, (c) dielectric with second ground plane, (d) die support, and (e) facet plates

The new cathode specifications and dimensional constraints are based upon the

L3 Technologies magnetron [41] which will be discussed later in the Design section. The cathode is designed to fit inside the anode of the L3 magnetron which will be placed in a vacuum chamber. In order to correctly align the cathode with the anode, an adjustable stand (Figure 18a) was fabricated which will also fit inside the vacuum chamber. Figure $18 \mathrm{~b}$ and Figure 18c shows the components of the electromagnet which houses the L3 magnetron with the experimental cathode. Figure 18d shows the full experimental setup. 

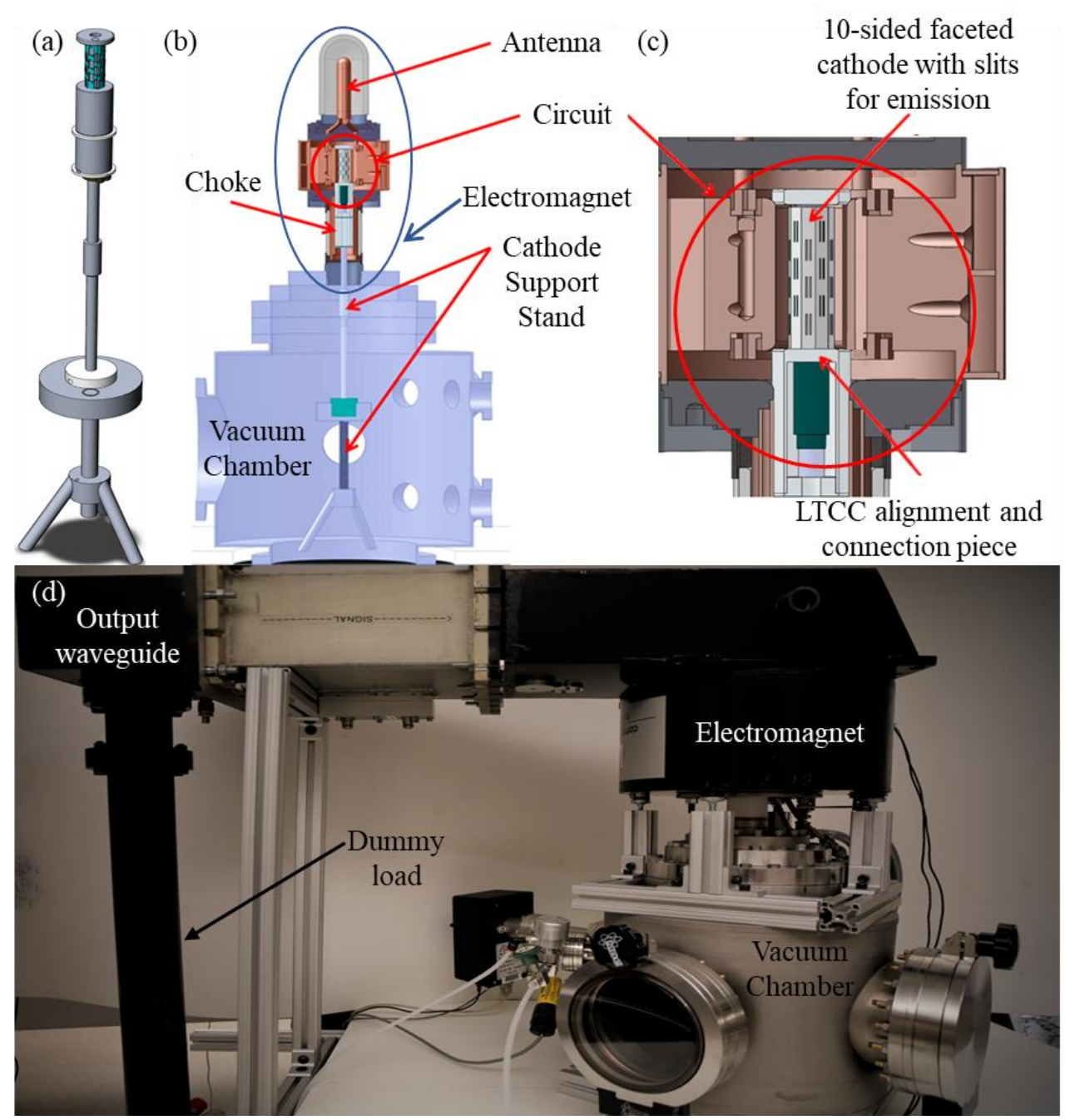

Figure 18. (a) A drawing of the cathode support stand which sits inside the vacuum test chamber, (b) drawing of the entire test chamber assembly showing the cathode support stand and faceted cathode inside the magnetron system surrounded by the anode circuit, (c) a close up drawing of the faceted cathode inside the magnetron circuit with an alignment piece to center the cathode inside the circuit, and (d) an image of the entire system showing the test chamber and the RF system components (electromagnet, dummy load, directional coupler, and coupling waveguide) courtesy of L3 Technologies. [41]

The new cathode offers a number of advantages over the traditional cathode. The field emitters can be turned off and on using the relatively low $(<60 \mathrm{~V})$ gate voltage. The spatial modulation may improve performance by injection of electrons at optimal locations to minimize startup times and increase efficiency. The temporal modulation could be used to control the electron spoke formation and allow for phase-locking [11]. 


\section{Modeling}

The new cathode structure design process required multiple iterations and models before finalizing the design. Modeling was done to compare the traditional, five sided, and ten sided cathodes $[12,13,14,15,16]$. The ten-sided cathode was chosen because simulations showed that the startup time could be reduced from 200 nanoseconds to 100 ns [12] and then again from $100 \mathrm{~ns}$ for continuous current to $35 \mathrm{~ns}$ for modulated current [14]. The simulations also showed that the modulated ten-sided cathode increased power density and allowed for active phase control during oscillation [14].

Figure 19 shows the VORPAL (Vsim) simulation model [12] of the cylindrical (Figure 19a), five sided (Figure 19b), and ten sided cathode (Figure 19c) in a rising sun magnetron. The rising sun magnetron was chosen because the primary mode of oscillation is the pi-mode; thus, the device can be modeled in $2 \mathrm{D}$ without strapping. Strapping essentially connects alternate anode cavities that are at the same RF potential with a wire or strap. These straps add extra capacitance to the resonator circuit which could be used to add some undesirable modes [42].

Table 1 shows the dimensions of the VORPAL model.
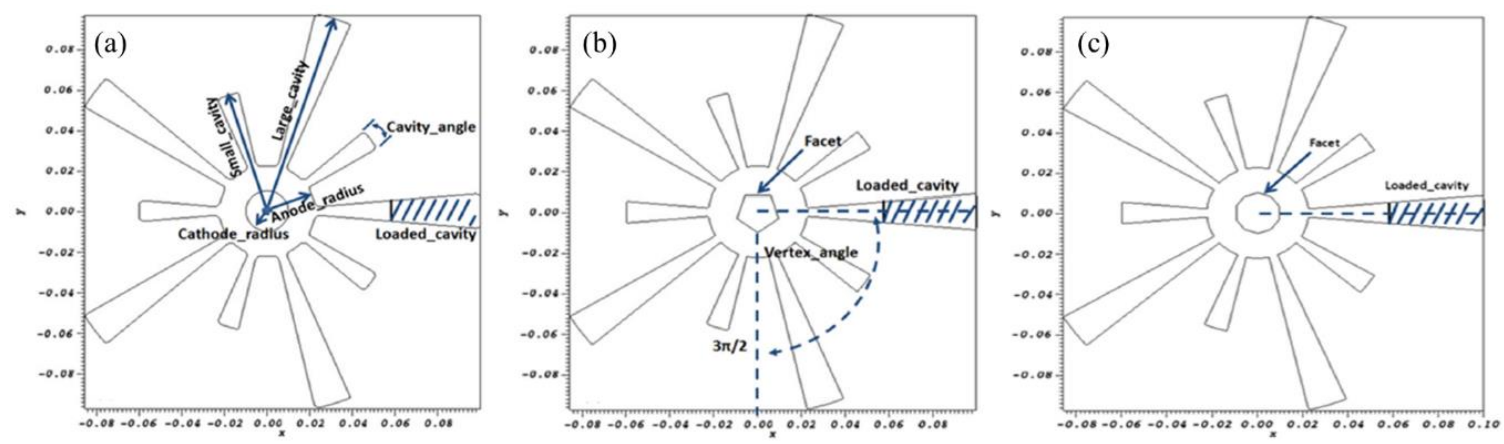

Figure 19. (a) Cylindrical, (b) five sided, and (c) ten sided cathode [12] 
Table 1. VORPAL simulation magnetron dimensions [12]

\begin{tabular}{|l|l|}
\hline Cathode radius $(\mathrm{cm})$ & 1.0 \\
\hline Anode radius $(\mathrm{cm})$ & 2.24 \\
\hline Facet width five sided $(\mathrm{cm})$ & 1.18 \\
\hline Facet width ten sided $(\mathrm{cm})$ & 0.618 \\
\hline Small cavity outer radius $(\mathrm{cm})$ & 6.0 \\
\hline Large cavity outer radius (cm) & 10.0 \\
\hline Cavity angle (degrees) & 10.0 \\
\hline
\end{tabular}

$\underline{\text { Materials }}$

The material system chosen for the new cathode was low temperature co-fired ceramic (LTCC), specifically DuPont 951 series [43]. LTCC is ideal for integrated microelectronic packaging technology, because of its rapid prototyping and low capital investment in equipment $[44,45,46]$. In the green, pre-fired state, the ceramic tapes consist of alumina particles, glass frit, and organic binder which makes them pliable and machinable. Features are created in each layer and then circuits are screen printed with metallic pastes. The layers are then laminated together and fired in a furnace at $\sim 850^{\circ} \mathrm{C}$. During the firing process, the organic binder volatizes, the glass flows, and the material hardens [47]. The complete manufacturing process is shown in Figure 20. 


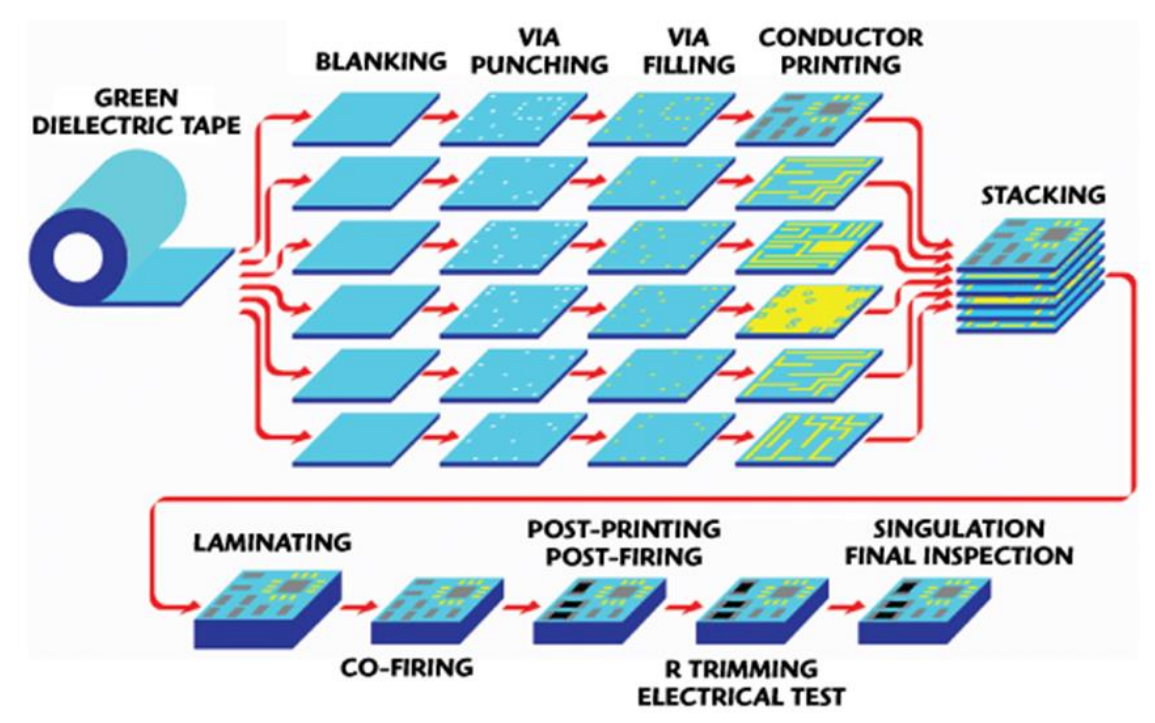

Figure 20. LTCC manufacturing process [48]

The advantages of LTCC are not only manufacturing process based, but

electrically, thermally, and mechanically as well. Electrically, LTCC has a low dielectric constant ( 7.8 at $3 \mathrm{GHz})$, low loss tangent $(0.006$ at $3 \mathrm{GHz})$, a very high current density of 3-D structures, and is easily integrated with passive components such as resistors, capacitors, and conductors. Thermally, LTCC has a high $\left(\sim 600^{\circ} \mathrm{C}\right)$ ambient temperature resistance and a very high thermal conductivity $(3.3 \mathrm{~W} / \mathrm{m} * \mathrm{k})$. Mechanically, LTCC in its green state is easily machinable (drill, cut, punch) and once fired becomes very strong structurally (230 MPa). The post fire shrinkage is $\sim 12.7 \%$ laterally and $\sim 15 \%$ vertically. The 951 series comes in 8 by 8 in $^{2}$ sheets of varying thicknesses. The sheets used for the cathode are DuPont 951PT and 951PX which are 114 um (5 thousandths of an inch mils) and 254 um (10 mils) thick respectively [43].

The co-fired silver pastes compatible with DuPont 951 series LTCC are vital to the design of the cathode in that each paste has a specific purpose. The transmission line and ground planes are composed of either Ferro 903-A [49] or DuPont 6145 [50], which are silver conductors having low fired resistivity $(<2$ and $<3 \mathrm{mOhm} / \mathrm{sq})$ and low 
viscosity ( 200 and $\left.\sim 160 \mathrm{~Pa}^{*} \mathrm{~S}\right)$. The connections between the transmission line and the die are composed of DuPont 6141 [51], which is a silver via fill conductor having low fired resistivity $(<3 \mathrm{mOhm} / \mathrm{sq})$ and high viscosity $(\sim 2100 \mathrm{~Pa} * \mathrm{~S})$. The SMA connector wire is connected to the cathode with DuPont 6146 [52], which is a solderable silver/palladium conductor having a high adhesion strength (>25 N initial) and low viscosity ( 180 $\mathrm{Pa} * \mathrm{~S})$. The $50 \mathrm{Ohm}$ resistor is composed of DuPont CF011 [53], which is a $10 \mathrm{Ohm} / \mathrm{sq}$ resistive paste having a low viscosity ( $90 \mathrm{~Pa} * \mathrm{~S})$.

\section{$\underline{\text { Design }}$}

As previously mentioned, the overall dimensions and constraints of the new cathode are based on the L3 Technologies magnetron [41]. The L3 magnetron cathode is a helical spiral of overall emission length $48.01 \mathrm{~mm}$ (1.89 in) and emission diameter 13.7 $\mathrm{mm}(0.54 \mathrm{in})$. These dimensions are constraints; however, symmetry is more critical rather than overall dimensions. The important characteristic of the cathode is that the cathode must be centered with the anode. Assuming both the anode and cathode are perfect circles and share the same center, this would be defined as concentricity. To achieve this, the cathode should be as close to a mathematically perfect circle for the entire length of the cathode as possible. Circularity and linearity requirements will be used and defined later. This is important for electron emission because the spokes that form will vary and be unstable with the geometry variations.

Another design constraint was the material. The LTCC sheets come in various thicknesses of 10,5 , and 2 mils which meant only certain thicknesses could be obtained. For simplicity, only 10 and 5 mil layers were chosen. The field emission die size (area of $2.5 \mathrm{~mm}$ by $8 \mathrm{~mm}$ ), also limited how many die could be placed within the emission 
bounds. In order to maximize emission area, three die were chosen per facet plate which meant a total of 30 die (60 emission sources). Four die would have been too difficult to connect to because the gaps left for vias would have been $\sim 2 \mathrm{~mm}$. This would have increased the likelihood of shorting and misalignment in addition to having 80 vias to connect to. Two die would have been insufficient because there would have been plenty of space for additional die. The gaps for vias would have been $\sim 8 \mathrm{~mm}$ which is the length of a die.

The facet plates fired thickness was chosen to be $1 \mathrm{~mm}$ (0.04 in) (5 10-mil layers) because this thickness is easy to manufacture and will give the necessary slot width to cover the emission areas of the die. Using a $60^{\circ}$ angle cut, and a $300 \mu \mathrm{m}$ top facet plate slot width, will give a $1.3 \mathrm{~mm}$ bottom facet plate slot width which will cover the $1.1 \mathrm{~mm}$ die emission area. This led to a non-faceted fired cathode OD of $11.64 \mathrm{~mm}(0.46 \mathrm{in})$. The next step was to design the location of the die as close to (without touching) the facet plates as possible, which was determined to be 4, 10 mil layers (spacer strips, then layers 7 to 5). The die must also connect to a ground plane which was determined to be 9.65 $\mathrm{mm}$ (0.38 in) OD (layer 4). This second ground plane OD, LTCC and ground spacing thickness, 60 vias, and the length of emission area was used to iteratively determine the stripline OD which was $9.14 \mathrm{~mm}$ (0.36 in) (layer 2), giving the final cathode an ID of $8.64 \mathrm{~mm}$ (0.34 in) (layer 1). The stripline characteristics are such that each revolution will be $1 / 4$ out of phase from the previous die. This will cause all three of the die aligned vertically to emit at the same time which will increase efficiency.

In order to obtain this length, the stripline was spiraled around the cathode in a barber pole design and the cathode was lengthened to $80 \mathrm{~mm}$ (3.15 in). The emission 
length however, is still the $48.01 \mathrm{~mm}$ (1.89 in) as previously mentioned. This additional length should allow for minor variations in the stripline design and will sit beneath the bottom end hat and be used to connect the cathode to the power supply. The transmission line (for the stripline) would start from the bottom of the cathode where the line would split into five sublines and then spiral around the cathode. Each of the five sublines would connect to six die each (totaling to 30 die) through holes in the top (outer) ground plane.

The five sublines would then merge back together and terminate into the 50 Ohm resistor. A power supply would connect to the transmission line through a subminiature version A (SMA) connector which would be soldered onto the bottom of the cathode. Figure 21 shows the circuit used to activate the field emitters (die).

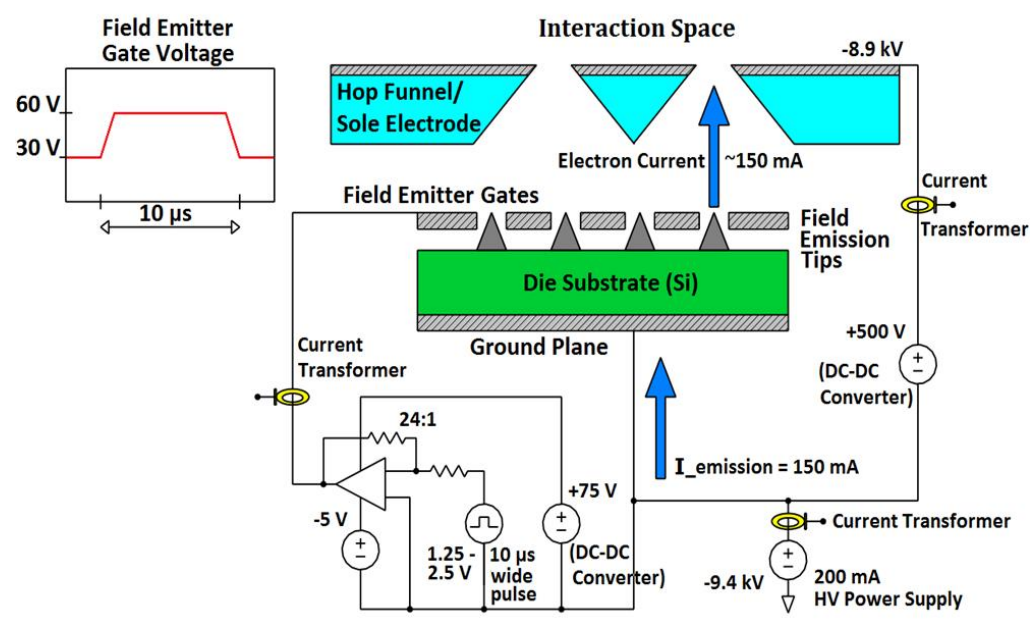

Figure 21. Circuit diagram of the gated field emission cathode drive scheme with a pictorial representation of the gated emitter structure and hop funnels (not to scale). All of the power supplies are external to vacuum and a pulsed RF source drives the field emitter gate at the desired frequency. A high voltage power supply floats the cathode power supplies and cathode structure at $-9.4 \mathrm{kV}$. [54]

In order to accommodate the stripline (transmission line) and internal electrical connections, the cathode will be composed of seven layers of low temperature co-fired ceramic (LTCC), rolled up into a tube shape starting from the inner layer 1 to the outer 
layer 7. There will be a silver paste layer on the inside of layer 1 ( 5 thousandths of an inch - mil) that will act as the first ground plane. Layer 2 (10 mil) will have the transmission line (stripline) screen-printed onto the outside of it, creating a 15 mil LTCC separation (dielectric) from the first ground plane to the transmission line. Layer 3 (10 mil) will have a screen-printed jumper on the inside, to connect the transmission line (outside layer 2) across the seam on layer 2. This layer 3 will have vias cut out of the LTCC to allow a connection from the transmission line to the die. This transmission line to die connection (tdc), is essentially holes in the LTCC layers (3 to 7) that line up and will be filled with silver paste. Figure 22a shows the three-dimensional new cathode design without die, spacers, or facet plates and Figure 22b shows a two-dimensional cross section of the cathode without the spacers or facet plates.

(a)

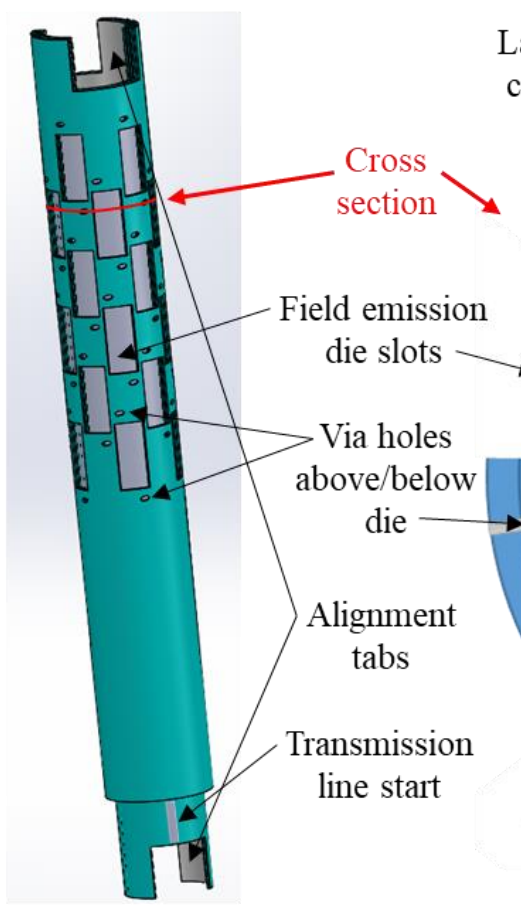

(b)

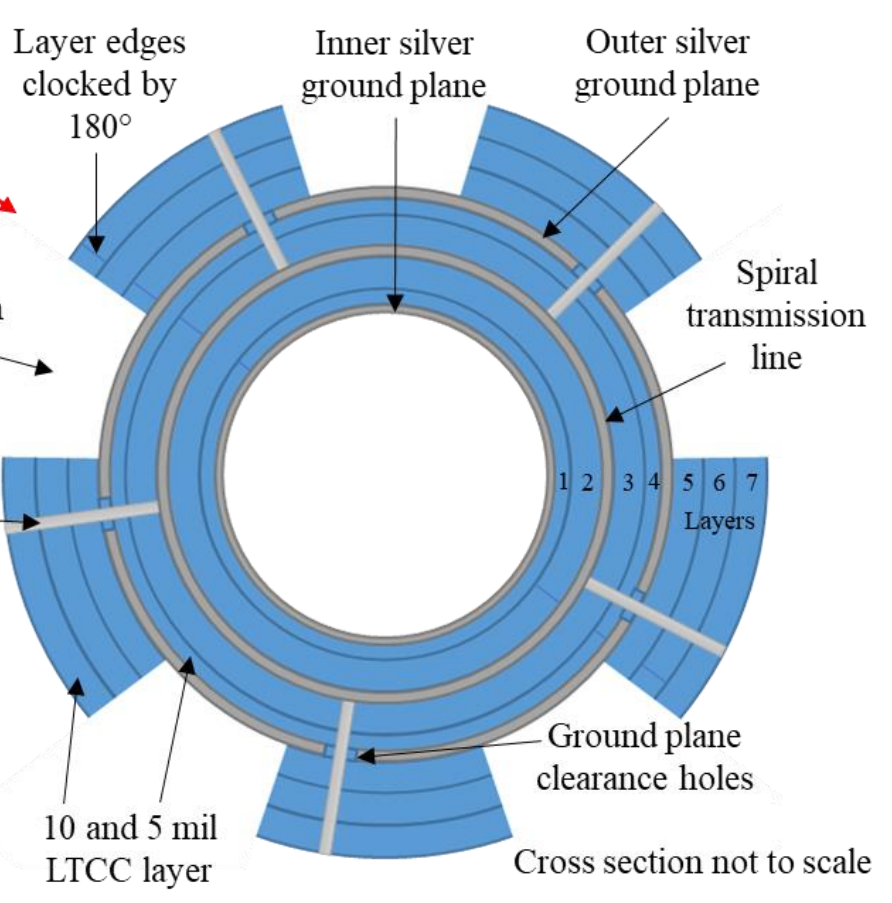

Figure 22. (a) 3-D cathode and (b) 2-D cross section 
Figure 23a, Figure 23b, and Figure 23c shows the outside and inside with paste of layers 1,2 , and 3, respectively.

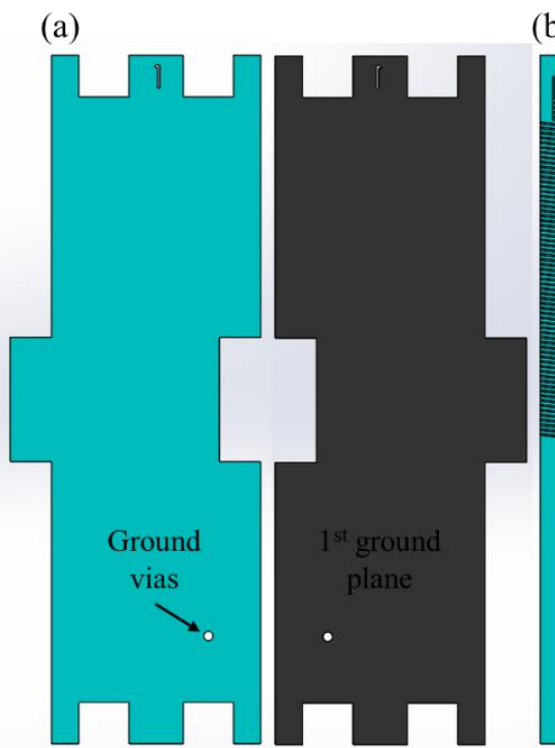

(b)

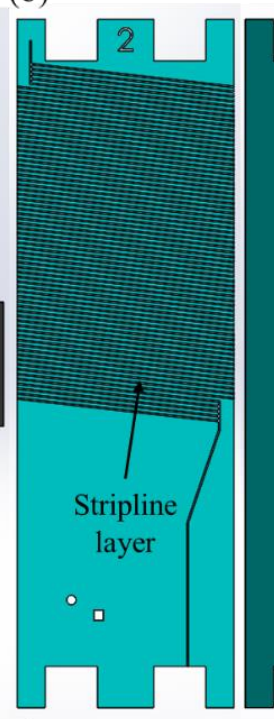

(c)
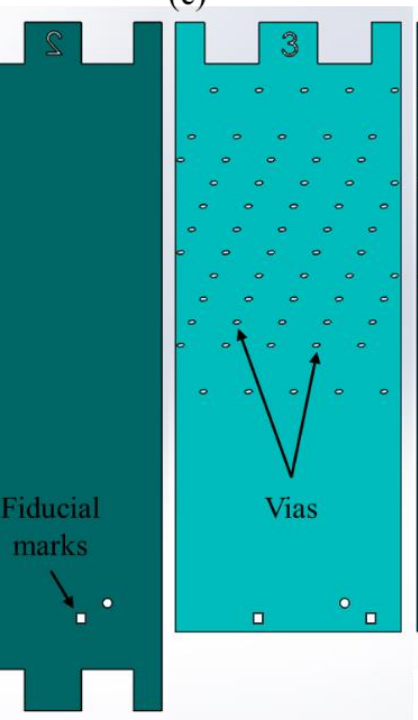

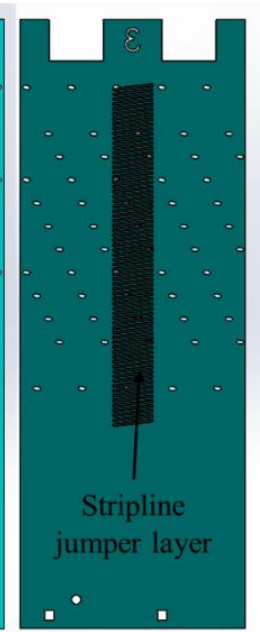

Figure 23. (a) LTCC layers 1, (b) 2, and (c) 3 outside and inside

Layer 4 (5 mil) will have a silver paste layer on the outside, to act as the second ground plane. Once again, another 15-mil gap (layers 3 and 4) from transmission line (layer 2) to the second ground plane. This layer 4 will also have vias cut out of the LTCC to continue the tdc. Because of the vias, the ground plane will have holes (ground plane clearance holes) in it to prevent shorting between the ground plane and the tdc (vias). The two ground planes will be connected by a single via (ground via), away from the others, from layers 1 to 4 . Layers 5, 6, and 7 will be primarily used for structural support. These layers will have vias through them to finalize the tdc and rectangular slots to hold the die in place. Figure 24 shows the LTCC layers 4 to 7. 


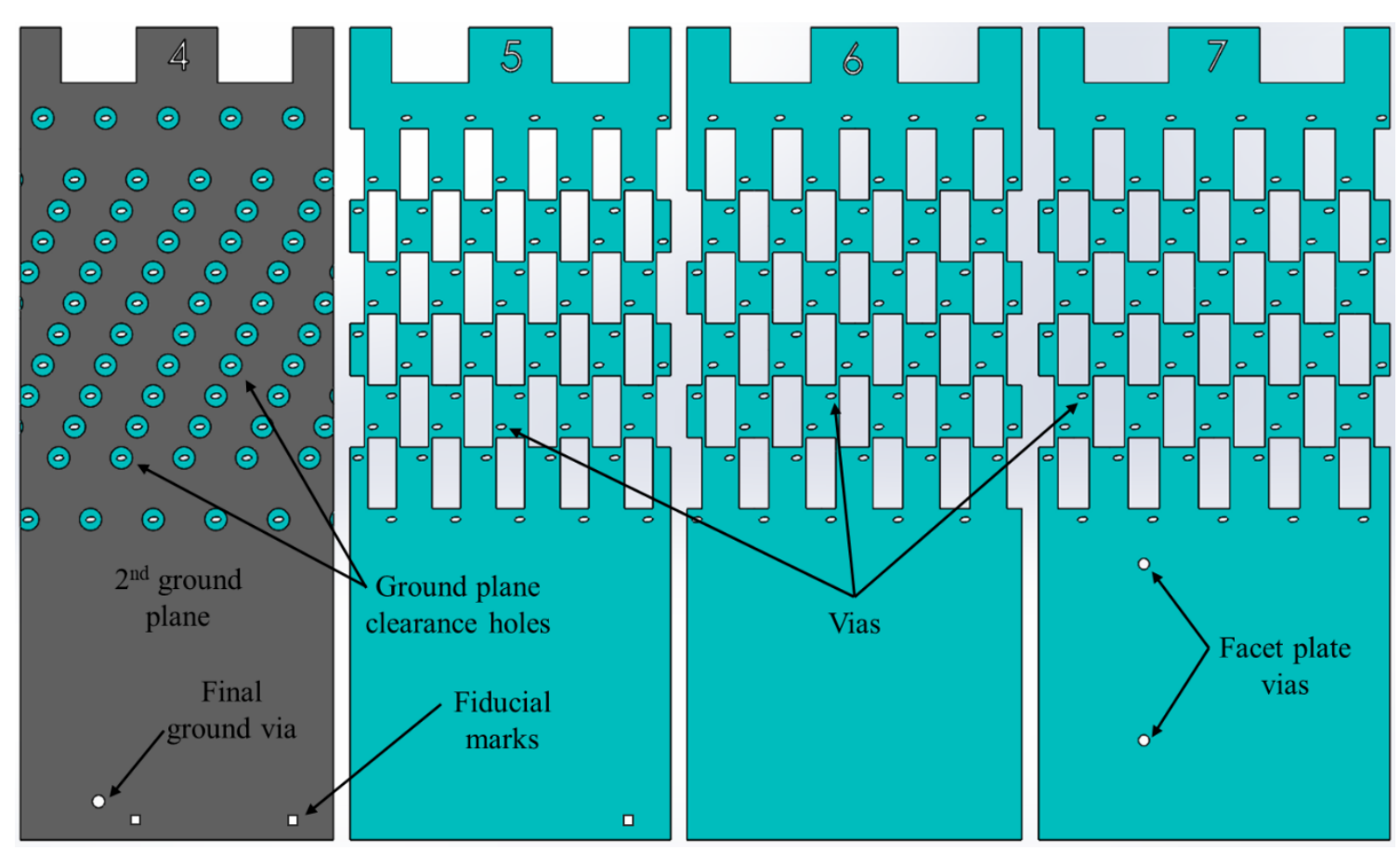

Figure 24. LTCC layers 4 to 7 (left to right)

The spacers will be used to separate the die from the facet plates which will be attached to the exterior of the cathode using ceramic epoxy as shown in Figure 26a and Figure 26b. These facet plates act as hop funnels (Figure 25) that funnel the electrons emitted from the die into a more controlled emission source [55]. In addition to increasing uniformity of electron emission, these hop funnels also protect the die from ion back bombardment which over time destroys the die.

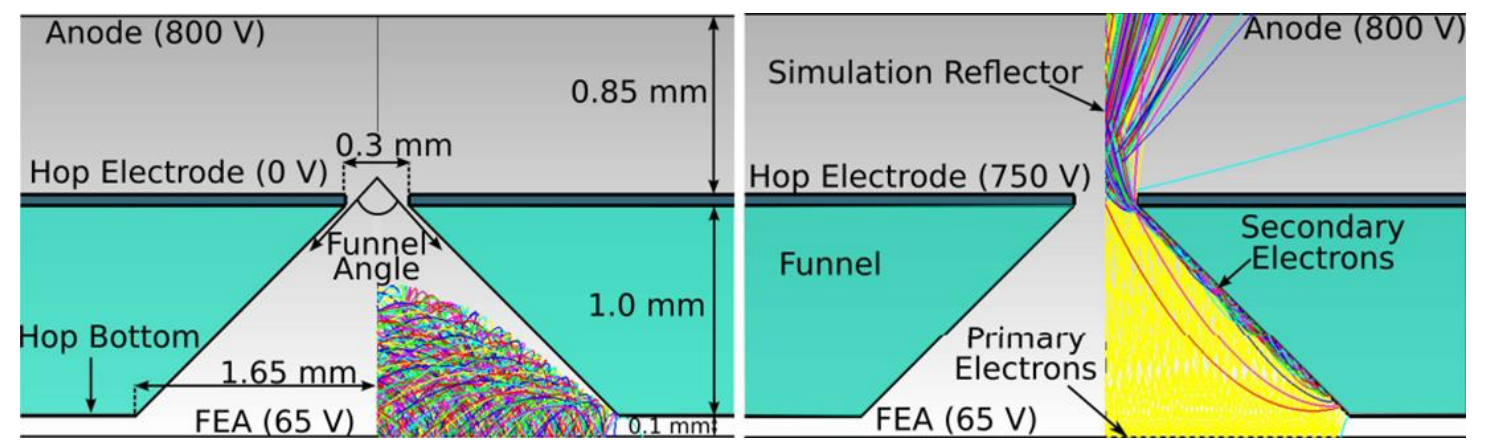

Figure 25. (left) Hop funnel primary and (right) secondary electron emission [55] 
(a)

(b)

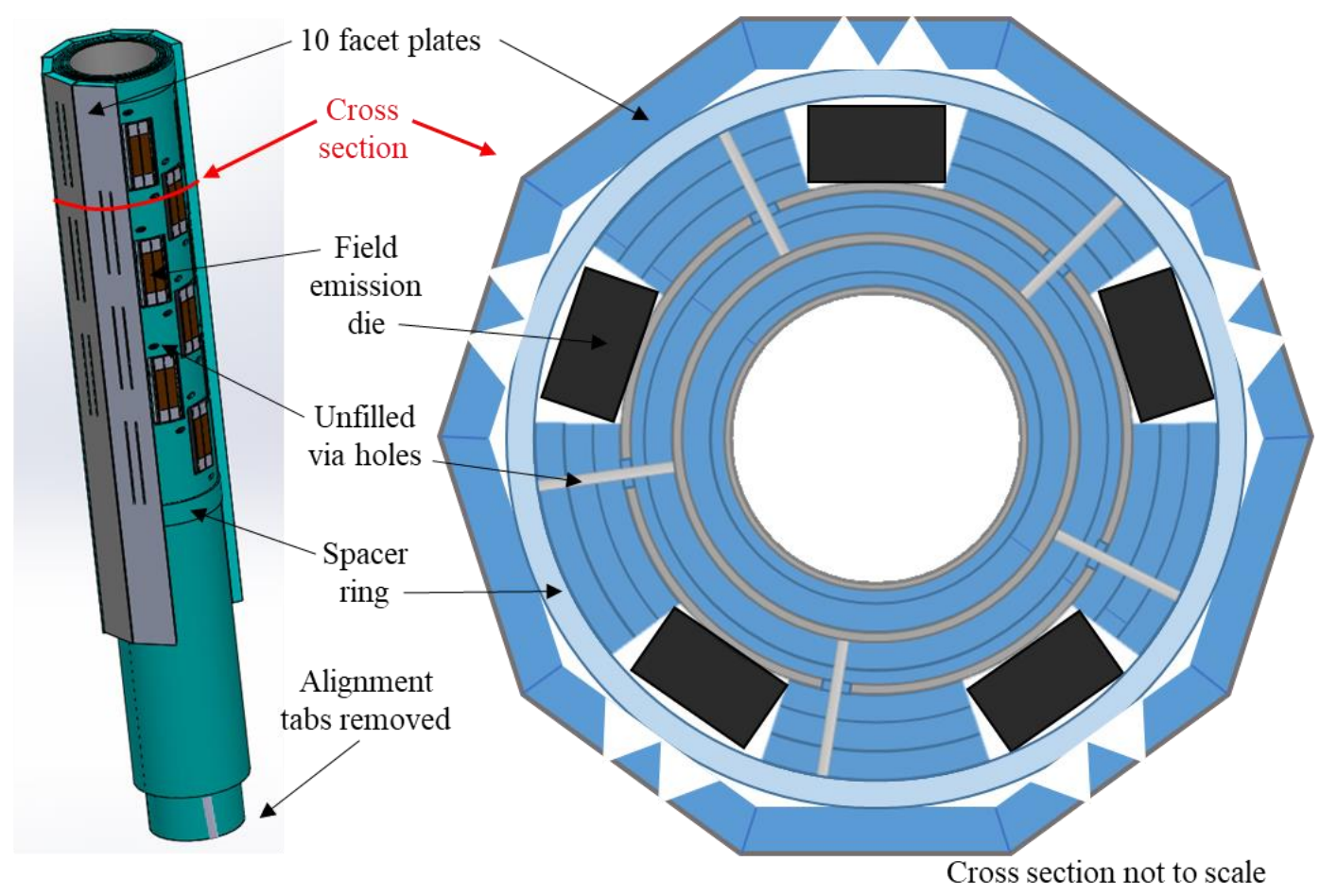

Figure 26. (a) 3-D cathode and (b) 2-D cross section

A general summary of every layer of the cathode is shown in Table 2.

Table 2. Summary of cathode layers

\begin{tabular}{|l|l|l|l|}
\hline Layer & Purpose & Features & Silver Paste \\
\hline 1 & $1^{\text {st }}$ ground plane & Ground via & Inside and via \\
\hline 2 & Stripline (barber pole) & Ground via & Outside and via \\
\hline 3 & Jumper layer (barber pole) & Ground/ trace vias & Inside and vias \\
\hline 4 & $2^{\text {nd }}$ ground plane & Ground/ trace vias & Outside and vias \\
\hline 5 & Die support/ via connection & Trace vias & Vias \\
\hline 6 & Die support/ via connection & Trace vias & Vias \\
\hline 7 & Die support/ via connection & Trace/ facet vias & Outside and vias \\
\hline Spacer & Prevent die contact with facet & None & None \\
\hline Facet & Prevent ion back bombardment & 'V' shape slits & Aluminum evap. \\
\hline
\end{tabular}




\section{LTCC Material Processing}

The newly designed cathode requires numerous advanced LTCC processing techniques. These techniques include: wrapping of LTCC, via filling, machining, maintaining geometric constraints while firing, and screen cutting/printing. The challenges that arise however, are that some of these techniques are either newly developed with minimal research or require additional expensive machinery to produce. The purpose of this research is either to develop new or to modify existing LTCC processing techniques in order to build the new cathode. A few of the current techniques are discussed below.

Wrapping

Wrapping or rolling the sheets of LTCC in the green unfired state is a relatively novel idea that has been used to produce ion mobility spectrometers [56] and electrostatic thrusters [57]. An ion mobility spectrometer (IMS) is a device which can identify and quantify compounds by ionizing them and transporting them down a drift tube with an electrostatic potential gradient as the driving force [58]. This device was created by rolling one continuous sheet of LTCC with tapered edges around a jig to achieve the multilayer structure. These tapered edges allowed for a smooth roll with a very gradual change in diameter in order to maintain roundness. If the edges were not tapered, once the first $360^{\circ}$ roll was completed, the tube would experience a very abrupt diameter change which would result in an oval shape rather than the desired circle. An example IMS and schematic of an IMS are shown in Figure 27a and Figure 27b respectively. 
(a)

(b)
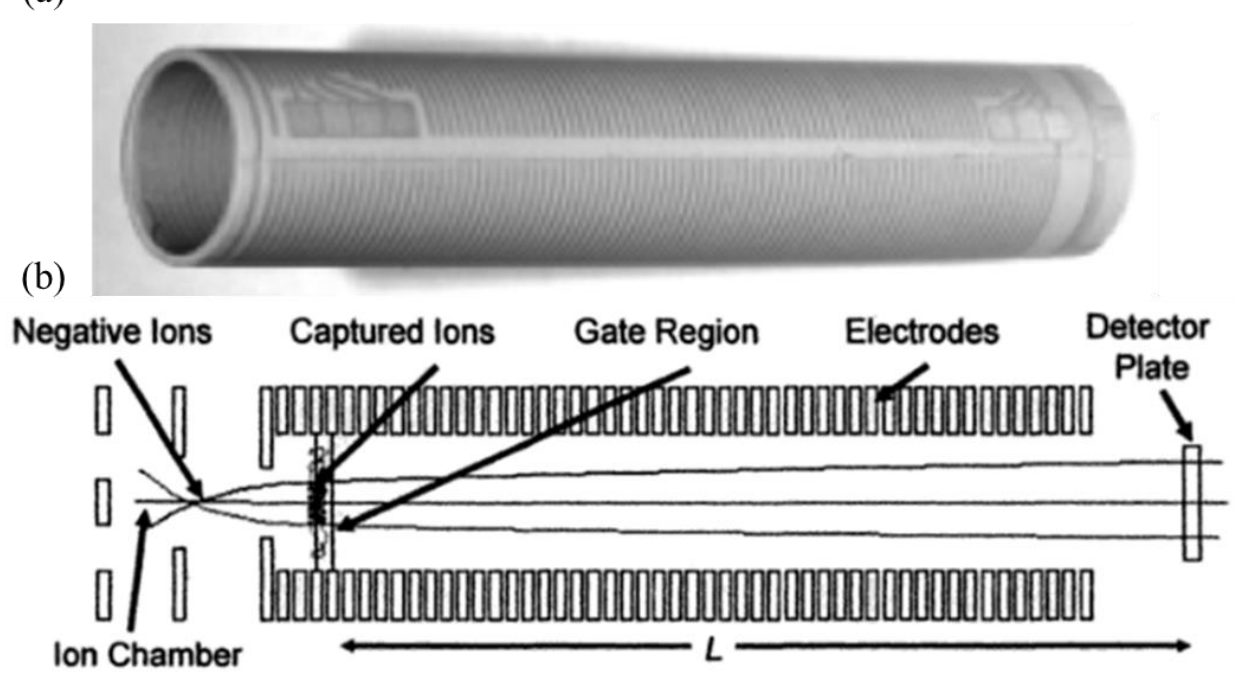

Figure 27. (a) IMS rolled structure [56] and (b) schematic [58]

The continuous strip design was utilized for the electrical interconnections between layers in two ways. The first was by printing paste on the front and back side of the strip which when rolled on top of each other would connect. The second was printing paste along the full length of the strip which, when completely rolled, would allow for the last layer and first layer to be connected. For this device, no vias were used between the rolled layers.

Electrostatic thrusters have been developed for various space applications including electrical-propulsion-based technologies such as resistojets, microcolloid thrusters, and micro discharge plasma thrusters [59]. The miniature ion thruster that used the LTCC system used an inductively coupled plasma (ICP) source for plasma generation [57]. This ion thruster was manufactured using LTCC, and the plasma containment tube was produced using a rolled LTCC technique. This miniature ion thruster is shown in Figure 28. 


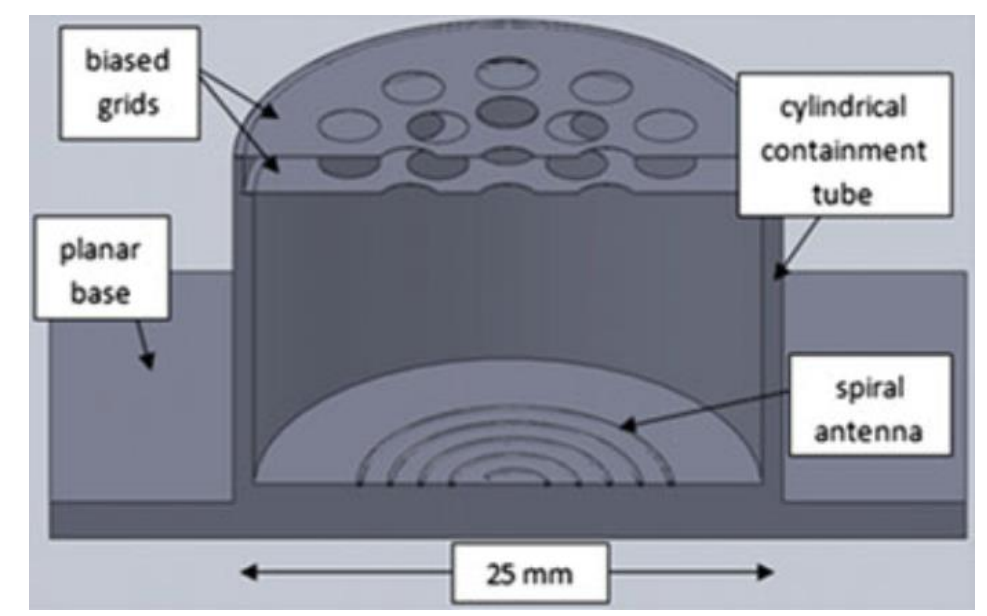

Figure 28. Cross section view of an ion thruster [57]

The rolling technique for this device used multiple continuous strips of LTCC that were wrapped around a jig. These strips were designed to wrap multiple times around the jig. For example, the tube structure was composed of 6 layers but used 4 strips. The first two strips would make up layers 1 to 3 ; and the last two strips would make up layers 4 to 6. This approach is different from the previous structure because only one strip was used for all of the layers in the IMS. This ion thruster device also used different electrical connections. Internal connections were made with paste on the strips that would connect to the base of the tube using electrical tabs or pads. Like the IMS, this device did not use vias to connect different layers of the rolled tube structure. The four strips of the device are shown in Figure 29a, and the device along with the electrical interconnection tabs on the base are shown in Figure 29b. 
(a)

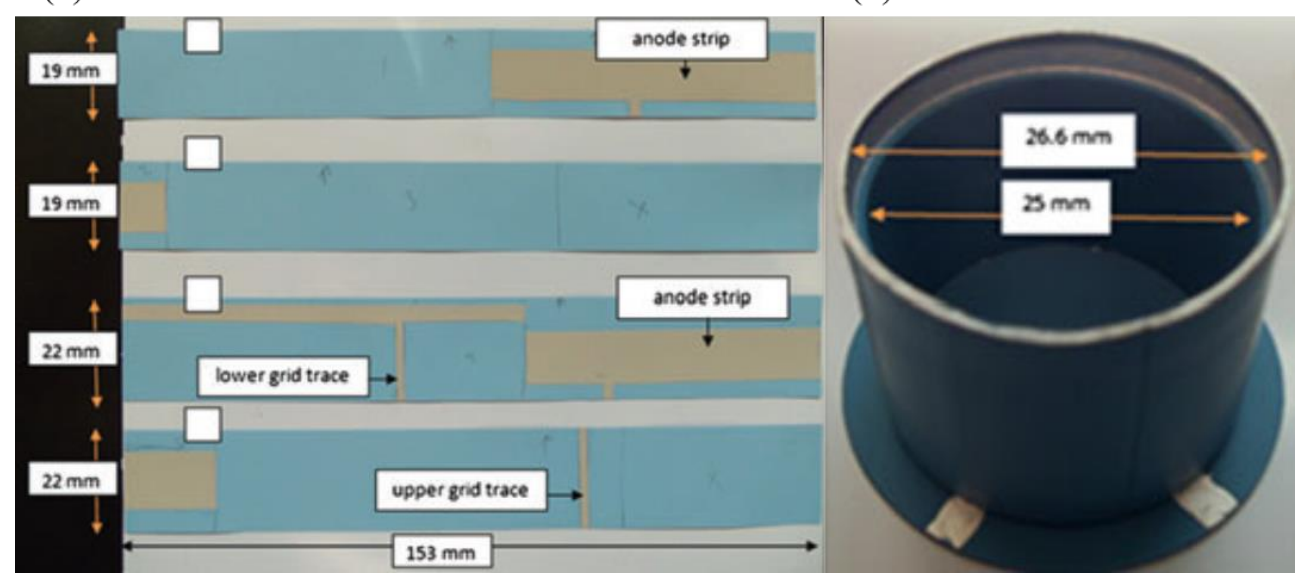

Figure 29. (a) Ion thruster strips and (b) device [57]

The single rolled continuous strip [56] and the multiple rolled individual strips [57] are excellent proof of concepts for the manufacturing of rolled LTCC layers. Both of these techniques wrap one strip of LTCC multiple times around the jig which allows for tight and consistent wraps nearly every time. The challenge for the cathode design; however, is wrapping seven single strips once around the jig, to obtain the seven layers. This increases the importance of achieving a tight wrap because if one layer is not wrapped completely around the jig, then the following layers will not wrap completely either. The cathode layers also contain 60 vias each, which makes alignment very crucial; whereas both of the previous wraps have zero vias per layer.

\section{$\underline{\text { Via Filling }}$}

Via filling is used in the LTCC system to electrically connect between layers. The vias or holes in each layer line up to create a tube or internally insulated electrical wire that connects layers to other layers [60]. The vias are usually punched using an USHIO [61] or Baccini [62] punch. These punching machines use various diameter circular punches that cut through the LTCC sheets to create the small holes or vias. In addition to punches, lasers have also been used to drill out the vias. Laser drilling is accomplished by 
repeatedly pulsing the laser at one spot until the laser breaks through the sheet of LTCC and creates a through hole [63]. This process of laser drilling; however, is very dependent on LTCC sheet thickness meaning that small vias (OD $<50 \mu \mathrm{m}$ ) are very hard to laser drill into thick sheets $(>50 \mu \mathrm{m})$. Figure $30 \mathrm{a}$ shows an example of a $50 \mu \mathrm{m}$ OD mechanical punch, and Figure 30b and Figure 30c show an example of a $100 \mu \mathrm{m}$ OD via made using mechanical punching and laser drilling respectively.

(a)

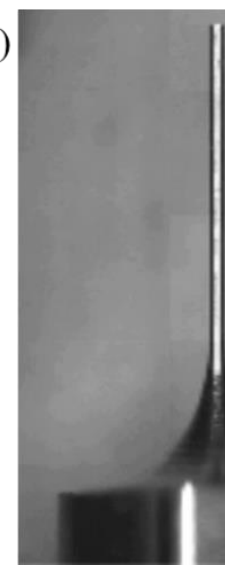

Figure 30. (b)

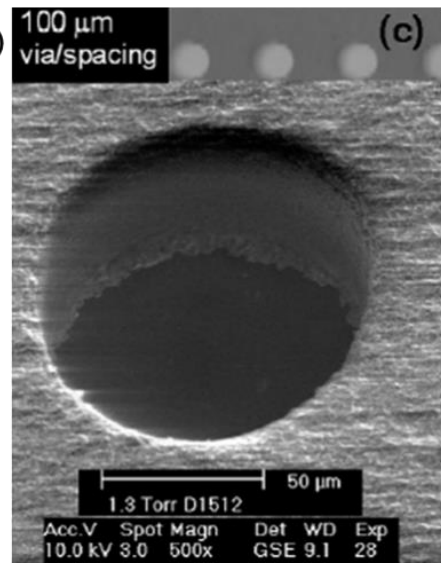

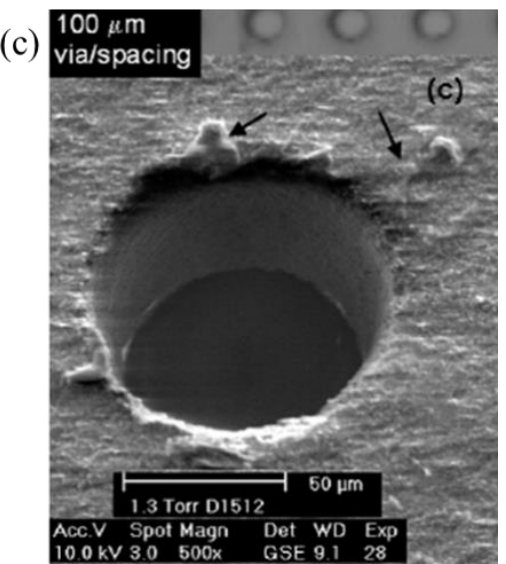

(a) $50 \mu \mathrm{m}$ punch, (b) $100 \mu \mathrm{m}$ via punched, and (c) drilled [63]

Screen printers are usually used to fill the vias after they have been formed. The LTCC sheet with the vias is placed on backing paper which is then placed on a porous stone. This porous stone allows for a vacuum to be pulled, holding the sheet in place. A stencil is used to cover all of the sheet except for the vias. The printer settings vary depending on the printer; however, the printer then squeegees paste along the stencil pushing paste into only the open vias. This squeegee goes back across the stencil again to ensure a complete fill of all the vias. If the printer does not have a built-in heater to dry the paste, the sheet and backing paper are then placed into a heater to dry the paste. Other printers use the vacuum to pull the paste into the vias and would not require the backing paper $[64,65,66]$. 
Another way to fill vias, without a printer, is to place the sheet with vias cutout onto a flat surface that has been covered with Teflon tape, no backing paper required. A clear plastic template or stencil that matches the vias is then laid on top of the LTCC sheet to align with the vias. The template and LTCC sheet may be taped down using low residue tape to ensure alignment of the vias. A plastic spatula is used to squeegee paste along the template until all of the vias are filled. After filling, the sheet is removed from the Teflon tape with a lateral slide across the flat surface to prevent the paste from adhering to the Teflon tape and pulling out of the vias. Lastly the sheet is placed in the heater to dry the paste, completely adhering the paste into the via [47].

The machinery is acceptable for precise and repeatable via filling results; unfortunately for this research there was no access to either of these punching or filling machines. Thus, the via filling had to be done by hand which is very effective when all of the materials are available. The challenge for the cathode design; however, is the lateral slide of the layers and the availability of silver paste. Some of the layers (5 to 7$)$ are composed of extremely thin $(\sim 400 \mu \mathrm{m})$ strips that hold the upper half of the layers together which tend to break if too much force is applied. Additionally, the lateral slide only works for specific via filling pastes which have a higher viscosity than screen printed pastes.

\section{Machining}

Machining or cutting of the LTCC sheets is fairly broad in process and technique. The sheets can be cut before lamination, after lamination, and even after firing. Although post fire cutting is generally more difficult, the shrinkage has already occurred thus giving a more precise cut. Some of the cutting techniques are hot knife cutting or 
scribing, dicing saw, ultrasonic cutting, and punching [67]. Hot knife cutting is used prefire and is generally used for very high-volume component sizes or for larger circuits with less stringent discrete component assembly requirements. Dicing saw is a common post fire technique that works very well for rectangular shaped parts. This technique produces tight outside dimensional tolerances and high-quality edges. Ultrasonic cutting can be used at any point in the LTCC processing; however, it works very well for post fire operation producing tight dimensional tolerances of unusually shaped parts.

However, this process is very expensive and very slow. Punching is used pre-fire and can easily and quickly accommodate unusually shaped parts; however, the tolerances are not so precise as some of the other techniques.

Other techniques for cutting the LTCC sheets are using a mill $[47,68,69]$ or laser $[57,70]$. Milling of LTCC is usually done pre-fire which gives extremely smooth edges but sacrifices dimensional tolerances because the shrinkage has not occurred yet. Post fire milling can be done; however, it is extremely difficult and will most likely result in cracking or breaking of the fired component, and the edges will not be smooth. The milling process is usually the removal of material from a moving object using a stationary cutter (tool). In some nontraditional instances, the object may be held stationary, and the cutter would move. The most common form of milling is end milling which uses a tool that can always cut in the horizontal direction (side to side) and sometimes (tool dependent) cuts in the vertical (axial, up and down) direction. The type of milling is almost always tool or cutter dependent. Figure 31a and Figure 31b show different milling cutter (tools) and a diagram of end milling respectively. 

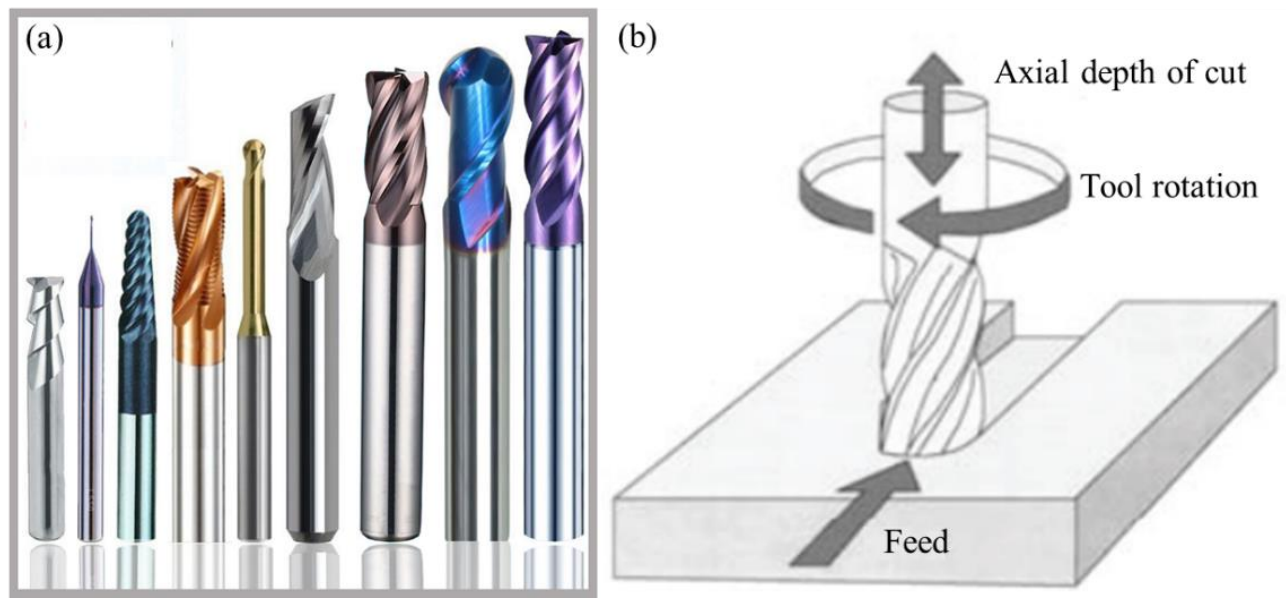

Figure 31. (a) Different milling tools [71] and (b) end milling diagram [72]

Laser cutting is very similar to milling in that it results in good edge quality but sacrifices dimensional tolerances when used pre-fire. When used post fire, it can produce tight tolerances; however, the edge quality is usually coarse. This technique is better than milling post fire because the laser is less likely to crack or break the fired component. As previously discussed, lasers can be used to drill via holes from about 75 to $500 \mu \mathrm{m}$ [70].

Figure 32a shows selected snapshots from a high-speed camera of $\mathrm{CO}_{2}$ ablation (removal of material) of green state HL2000 LTCC at $0.15 \mathrm{MW} \mathrm{cm}^{-2}$ and $100 \mu \mathrm{s}$ pulse duration. The grid spacing is $100 \mu \mathrm{m}$, and the beam diameter and ablation site (AS) are shown where the time stamps are in $\mu$ s [70]. Figure 32b shows how a laser cutter works using the focal length (point) to precisely cut the material. 
(a)

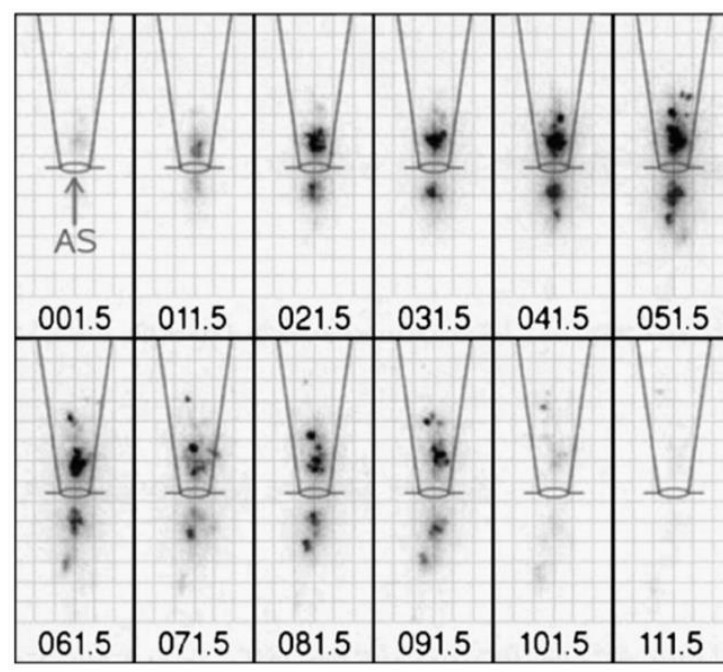

(b)

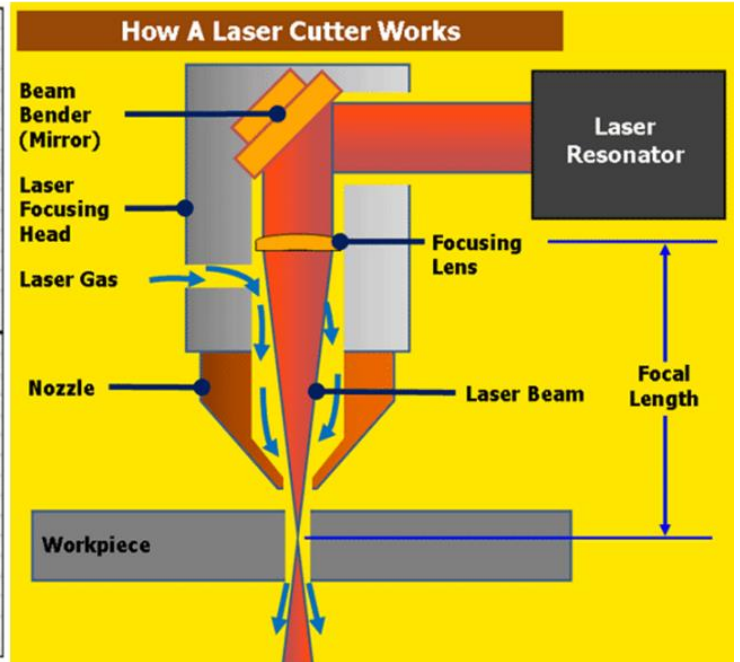

Figure 32. (a) Snapshots of a laser pulse [70] and (b) how a laser cutter works [73]

Both of these methods were compared and evaluated for the cathode manufacturing process and the results can be found in Chapter 3.

\section{Firing Control}

It is very important to account for the shrinkage and warpage that occurs when the LTCC material system (including silver paste) is fired. In addition to adding $13-15 \%$ on all of the dimensions, the geometric shape of features must also be considered. One example that will be used for this research is circularity of the cylindrical structures which is defined as the maximum outer diameter (OD) minus the minimum OD. More specifically, maintaining circularity of rolled devices using LTCC sheets is an important feature.

Two examples of techniques to maintain circularity for rolled structures were used in the devices previously discussed. The ion mobility spectrometers fabrication showed that there exists a diameter gradient along the tube from top to bottom [58]. The bottom (tube contacts the setter sheet) had the largest diameter and the top (free end) had 
the smallest diameter. The middle of the tube had the most consistent diameter which was within $0.5 \%$ of the mean diameter. The researcher's solution was to over build the tube length $(10 \mathrm{~cm})$ and then remove the bottom $(0$ to $\sim 2 \mathrm{~cm})$ and top ( $\sim 8$ to $10 \mathrm{~cm})$ ends of the tube. This resulted in a designed $6 \mathrm{~cm}$ (original 2 to $8 \mathrm{~cm}$ section) length tube of a diameter within $0.5 \%$ of the mean diameter.

The electrostatic thrusters [57] took a different approach to solving this circularity issue. It was determined that circles cut out of flat sheets maintain circularity; however, rolled sheets to obtain circles do not (wrapping sheets around a circular jig). This information was used to build caps that would be placed inside the tube while being fired. These caps consisted of multiple large and small outer diameter (OD) layers that were cut out from the sheets of LTCC. The smaller diameter circle layers were stacked on top of the larger diameter circle layers and laminated together. This cap building process is shown in Figure 33a. This forms a cap that can fit inside the tube (smaller cap OD equals tube ID) and have a base (larger OD) that keeps the tube upright. These caps were sprayed with Boron nitride to prevent bonding to the tube during firing. One cap was placed on the bottom and one on the top of the tube, and then the tube was placed upright in the furnace to be fired.

The results obtained circularity measurements consisting of max OD minus min OD of less than $0.44 \mathrm{~mm}$ with silver paste and even better without paste which was well below the $0.6 \mathrm{~mm}$ target. It was also shown that tubes consisting of six plus layers without a top cap, only had slight variations, compared to six minus layers with a top cap. Some examples of bad and good circularity are shown in Figure 33b and Figure 33c, respectively. 


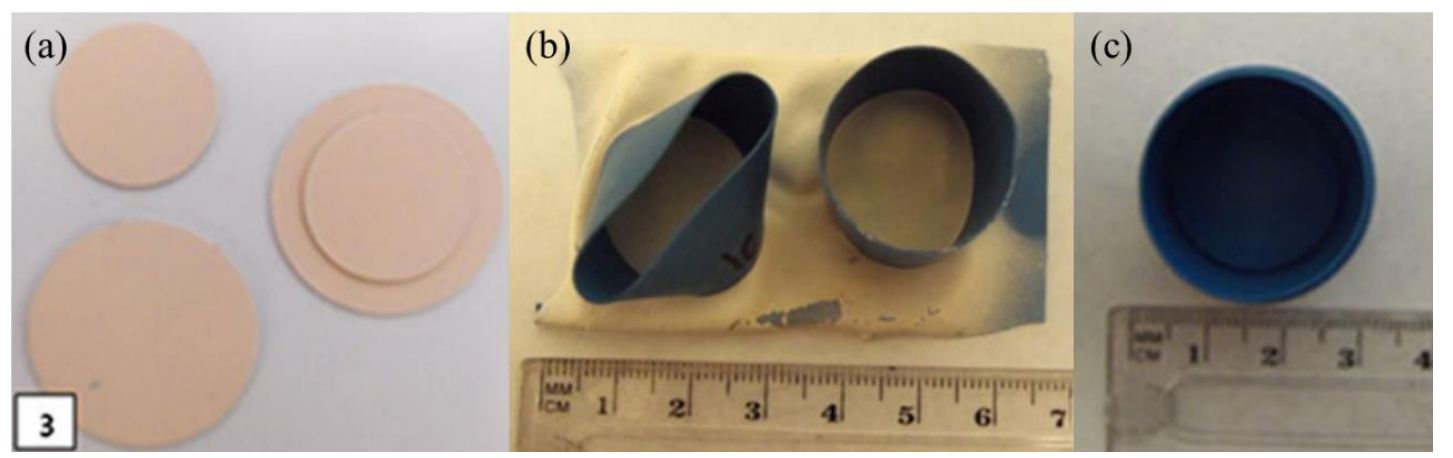

Figure 33. (a) Building of a cap, (b) examples of bad, and (c) good circularity [57]

The material removal technique [58] and cap technique [57] can achieve measurements within $0.5 \%$ mean diameter and circularity measurements within $0.44 \mathrm{~mm}$ ( $0.8 \%$ mean diameter) respectively which validates their effectiveness. The cap technique was included in the experiment for maintaining firing control because the caps were easy to manufacture and implement with the other techniques. The material removal technique was not included in the experiment because it would have required additional post fire processing and a complete modification of the original layer design which was beyond the scope of this research.

\section{$\underline{\text { Screen Cutting }}$}

Screen cutting and printing is very important for applying the various pastes onto the LTCC material. For example, the resistive paste is documented as Ohms/sq which means the resistance is directly related to its physical dimensions. In order to achieve these accurate dimensions, thick screens are used to cut templates that will be used to print the paste. These screens are composed of various meshes (wire OD, mesh opening, $\%$ open area, etc.) that are tightly glued to a frame. A liquid emulsion is coated directly on the mesh of varying thicknesses ( 0.0001 to $0.002 \mathrm{in}$, using 0.0001 in increments and 0.002 in above with $10 \%$ error) [74]. 
The emulsion is UV curable which means it hardens when exposed to light. Traditional screen cutting uses a template or stencil that matches the desired paste shape or area. This template is placed on top of the screen which is then exposed to light. This hardens all of the emulsion except where the template was placed. After exposure to light, the screen is washed which washes away the unexposed emulsion, exposing just the mesh in the shape of the paste template. At this point, the screen has exposed mesh in the desired paste shape, which paste can go through, and hardened emulsion everywhere else [74]. This process gives very sharp edges between the hardened emulsion and empty mesh but is costly in that the screen; once exposed, can only be used once. The frames can be re-meshed and new emulsion can be applied, but this requires sending out to a manufacturer. A cross section view of the emulsion and mesh is shown in Figure 34.

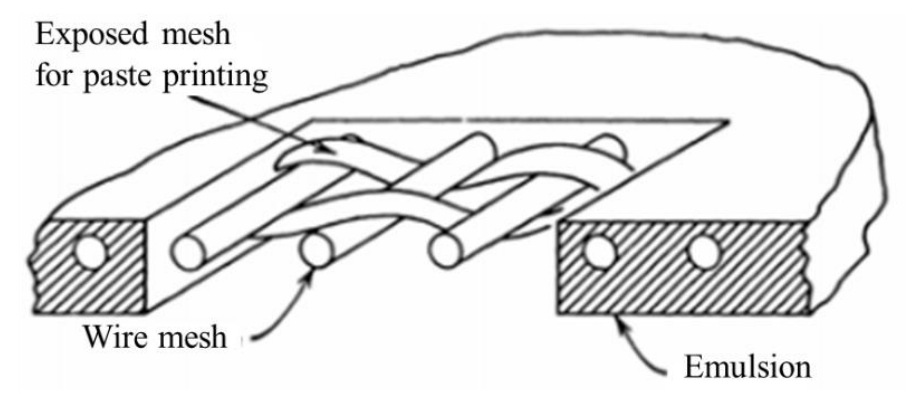

Figure 34. Example of emulsion and mesh area [75]

Another process for cutting the screens is by means of a laser [57]. The hardened emulsion can be burned away by a laser. Similar to milling out material, the laser burns the emulsion in the desired paste shape leaving only the mesh exposed. This process is very precise but not so precise as UV exposing because the edges are not smooth and the cut is not perpendicular to the mesh all of the time. In practice, the smallest line width achievable with a laser was $\sim 70 \mu \mathrm{m}$. For certain large-scale applications this is 
exceptional, giving the ability to reuse screens extremely quick. However, when desired line widths are $\sim 130 \mu \mathrm{m}$, the lack of perpendicular cuts can affect the printed widths.

The printing process through these screens usually requires printing machines to ensure even thickness of deposition. The screen is held above the LTCC sheet; paste is applied to the screen, and then a squeegee travels over the top of the screen with enough pressure to contact to the sheet. This process ensures the paste transfers through the screen onto the sheet at the desired thickness of the emulsion, and then tension of the screen ensures immediate 'snap off' of the screen preventing the paste from sticking to the mesh. This process is shown in Figure 35.

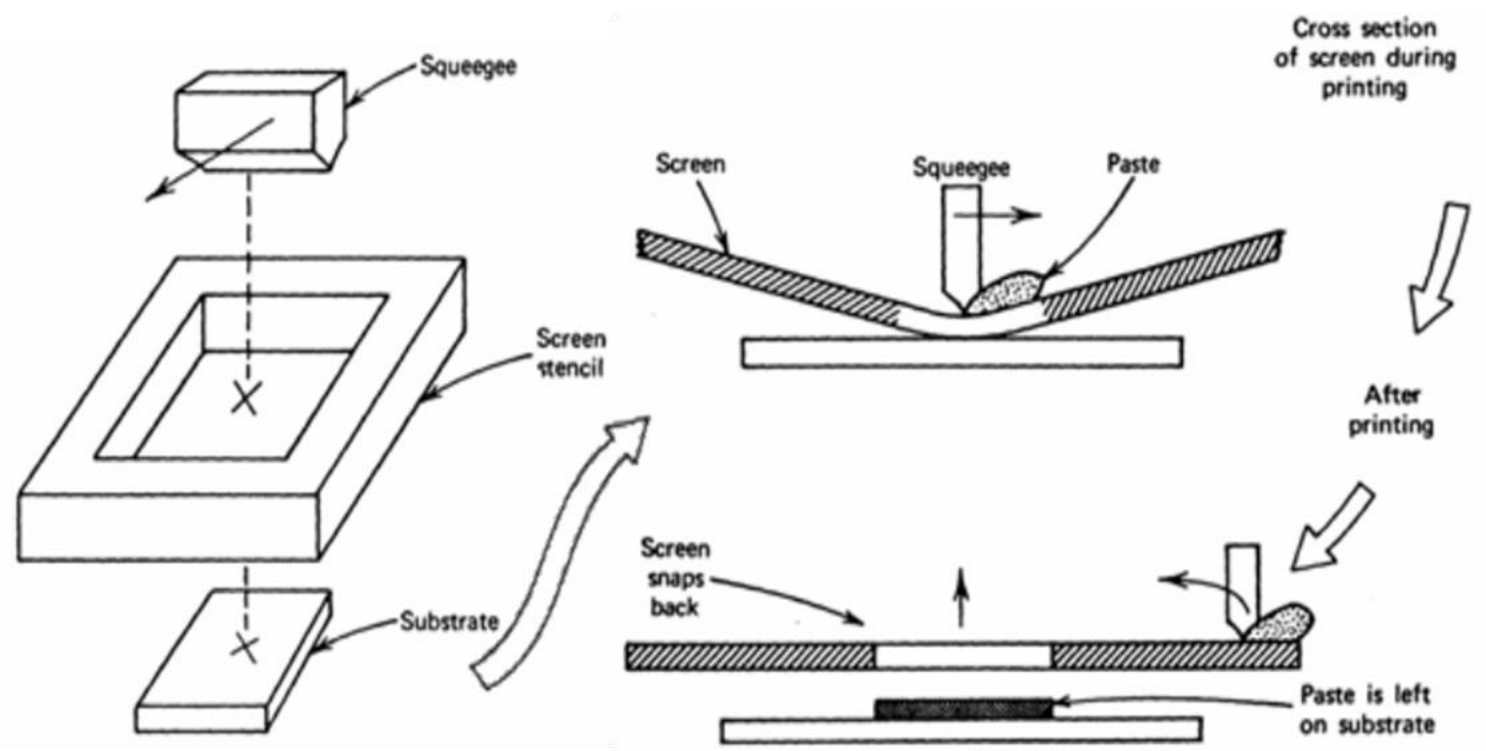

Figure 35. Example of screen printing [75]

The previously discussed techniques have been used thus far to manufacture micro electro mechanical system (MEMS) devices in LTCC. The rolling techniques have proved useful in rolled structures without vias to achieve a desired geometry. The via filling techniques have been used on flat structures with the help of machines and 
equipment. LTCC machining, various firing control techniques, and screen cutting/printing were also introduced.

The difference between the cathode structure that warrants additional techniques is the introduction of vias, the stripline, and facet plates. The vias and one layer per roll requires techniques that can easily wrap the layers tightly while maintaining alignment. The via filling of rolled devices requires techniques that work for sensitive layers and various silver pastes and for complete via fill. The machining techniques previously discussed will be implemented for facet plate manufacturing. The cathode fired structure requires techniques that can achieve the desired geometry (circularity and linearity) rapidly. Lastly, for rapid prototyping, screen cutting/printing must be produced in house with the equipment available. 


\section{CHAPTER THREE: TECHNIQUES}

This chapter will discuss the LTCC rolling/wrapping, via filling, machining, and firing techniques that were compared. The LTCC wrapping techniques that were compared are the layer by layer technique which wraps each individual layer at a time and the layer by group technique which wraps multiple layers at a time. The via filling techniques that were compared are the flat fill technique which fills each layer of the vias at a time and the pneumatic pump technique which fills each via at a time. The machining techniques that were compared are the laser and mill techniques, which use a laser and mill respectively, to remove the green (pre-fired) LTCC material. The firing techniques that were compared are horizontal, caps, stand, and stand with caps.

All of these techniques previously mentioned directly correspond to manufacturing steps for producing a cathode structure which is why they were chosen. The cathode structure manufacturing process consists of:

- All seven layers of LTCC are cut, cleaned and conductive paste is applied to the layer surfaces and vias (via filling). These vias complete the connection from the stripline to field emission die, also known as the transmission line to die connection (tdc).

- All seven LTCC layers are rolled onto the jig (LTCC wrapping) and then laminated into the cathode structure.

- The electron hop funnels (facet plates) that protect the die and increase uniformity of the emission source are manufactured. 
- Lastly, the cathode structure (firing technique) and facet plates are placed into a furnace where they are fired.

\section{LTCC Wrapping}

LTCC wrapping or rolling techniques are implemented when wrapping the seven

layers of the cathode. As previously mentioned in chapter 2, rolling LTCC has proved useful for fabricating tube-like structures. An alternative to rolling, is cutting circular washer like layers and stacking them to the desired height. This stacking technique cuts each layer to the desired diameter and then stacks each layer to obtain the correct height. The rolling techniques on the other hand, cuts each layer to the desired height and then rolls to obtain the correct diameter. Depending on the application, both of these techniques can be very effective and simplify the manufacturing process.

Wrapping was used instead of stacking circular washers because stacking leads to cracking in devices of similar geometry to the cathode. In addition to cracking, rolling simplifies the design to seven rolled layers, as opposed to hundreds of stacked layers. These differences are evident when comparing the rolled ion mobility spectrometers (IMS) $[56,57]$ previously discussed, to a stacked IMS [69]. The rolling technique also produces extremely smooth surface edges; whereas stacking leaves ridges. For the internal stripline, the stacked method would have added 100's of vias which would have increased the probability of shorting or creating an open circuit. Figure 36 shows a quarter cathode structure model, of the rolled method compared to the stacked method. 


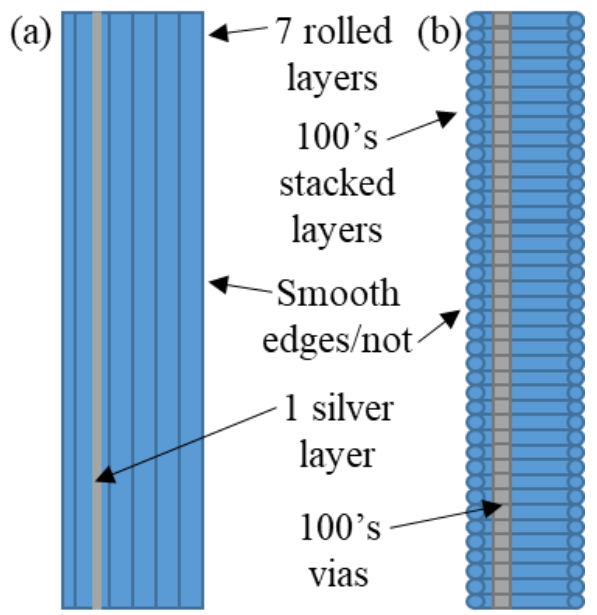

Figure 36. (a) Quarter model rolled and (b) stacked cathode

The design of the cathode structure and internal stripline, as previously mentioned, requires each layer to wrap once, completely around the cathode. This means each layer has a designated surface length which directly relates to the diameter of the cathode. If one layer does not wrap fully (seams meet), then the following layer will not wrap fully and the error (looseness) get worse with each wrap. If the layers do not align, the vias will not align either, and the cathode will fail. The problem with rolling LTCC is that accurate alignment of layers becomes essential for a successful cathode structure. An example of a tight (correct) wrap and a loose (incorrect) wrap are shown in Figure 37a and Figure $37 \mathrm{~b}$ respectively.
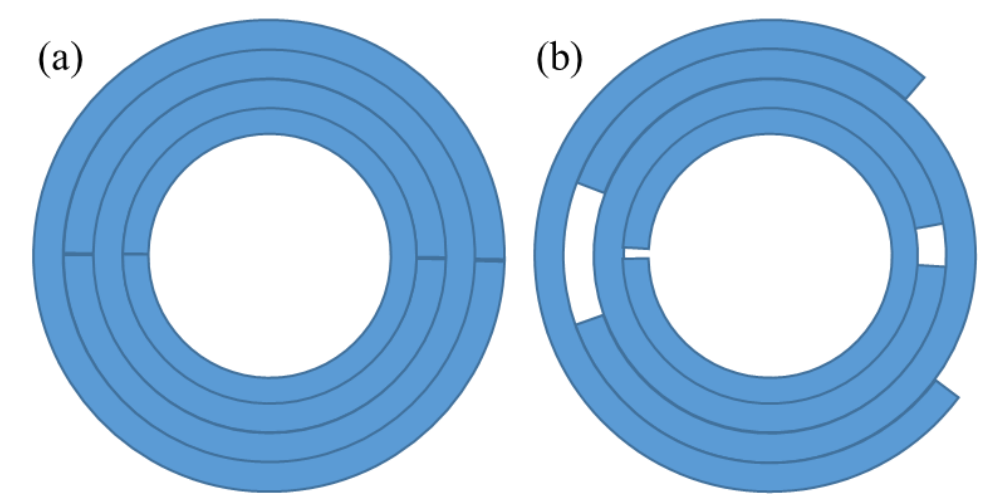

Figure 37. (a) Tightly wrapped and (b) loosely wrapped example cathode 
For both techniques discussed below, the layers are wrapped around a jig of diameter $9.728 \mathrm{~mm}(0.383 \mathrm{in})$ and then laminated. The lamination process for DuPont 951 series $\mathrm{LTCC}$ is pressed under $20.7 \mathrm{MPa}(3 \mathrm{ksi})$ at $70^{\circ} \mathrm{C}\left(158^{\circ} \mathrm{F}\right)$ for $10 \mathrm{~min}$ [43]. To maintain equal pressure on all surfaces, the lamination equipment used is an isostatic press. In order to validate the jig diameter, it was found that LTCC begins to crack when the surface length of the sheet is $<6 \mathrm{~mm}(0.24 \mathrm{in})$, or when wrapping on a circular jig of diameter $<1.9 \mathrm{~mm}(0.075 \mathrm{in})[76]$.

\section{Layer by Layer}

The layer by layer technique is the process of wrapping one layer on the jig at a time and then laminating after all seven layers have been wrapped. Layer 1 is wrapped and bonded to the jig using poly 2-ethyl-2-oxazoline (PEOX). PEOX is a glue that is used to bond LTCC layers in the green state with minimal lamination pressure. Layer 2 is laid out flat, and PEOX is applied to the surface, near the edge used to align with layer 1 . The jig is clocked $180^{\circ}$ so that the seams of layer 1 and 2 do not align. The jig with layer 1 is then placed on layer 2, using the alignment tabs to align, and held down until the PEOX dries ( $\sim 30$ seconds) as shown in Figure 38. More PEOX is applied to the other surface near the edge of layer 2 and then the jig is rolled, wrapping layer 2 onto the jig. The process is then repeated for all of the remaining layers, all the while maintaining a clocked $180^{\circ} \mathrm{jig}$, so that the seams between layers do not overlap and touch. Finally, the spacer strips are rolled on using PEOX, and the cathode structure is placed in the isostatic press to be laminated under normal conditions previously discussed. 


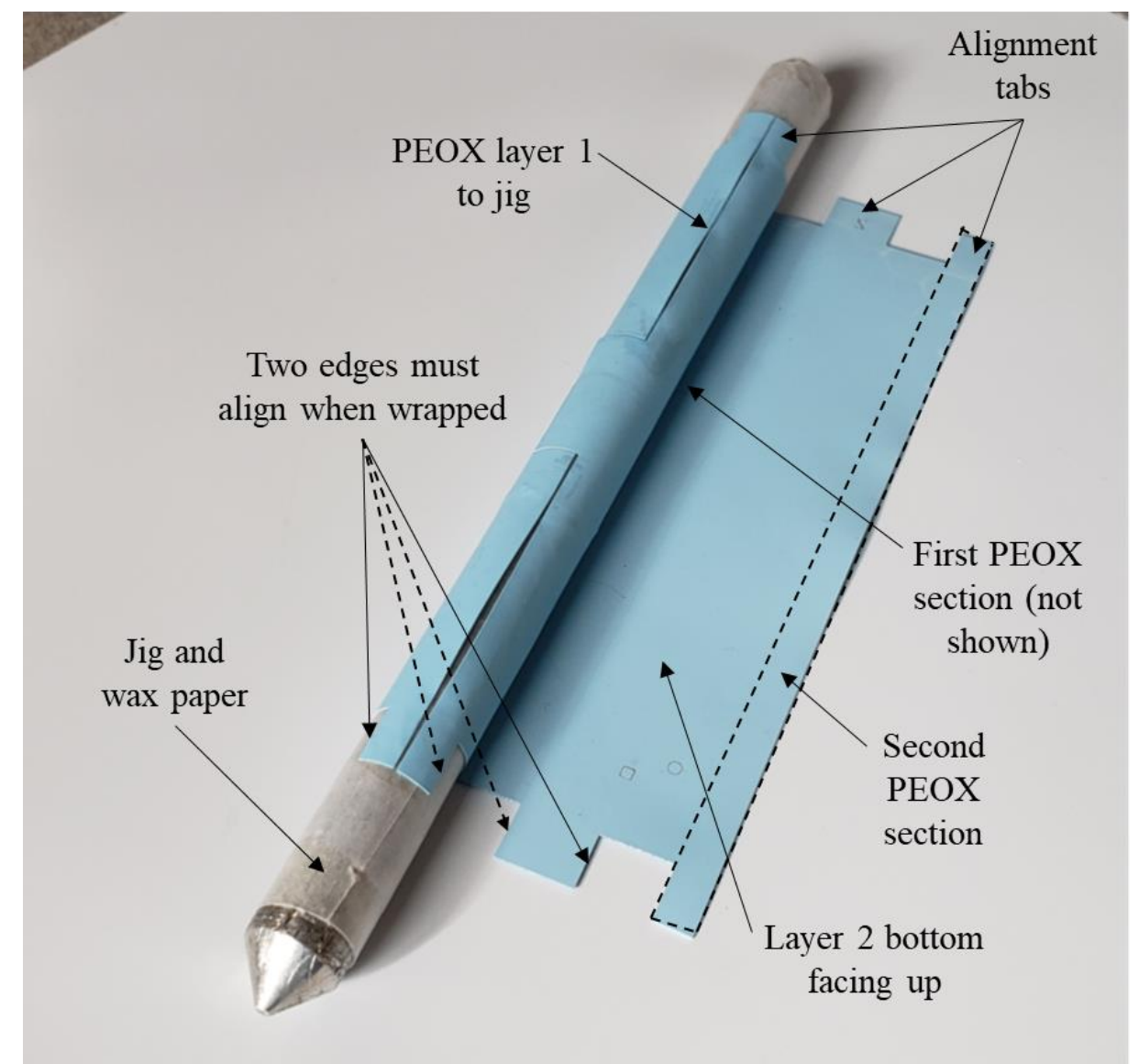

\section{Figure 38. Layer by layer technique: layer 1 has been completely wrapped around the jig and layer 2 is about to be wrapped}

This layer by layer technique is optimal for sensitive layers, such as layers 5 to 7 , because it reduces stress on the layers. "Sensitive layers" refer to any layers that cannot be subjected to tension or repeated bending. The rectangular sections containing the vias on layers 5 to 7 tend to break off if the layer is rolled more than once. This can be remedied by using PEOX to glue the rectangles back on the cathode, but this fix usually leads to misalignment and unconnected vias.

This technique is not optimal for strong layers (can withstand tension and bending), such as layers 1 to 4 , because the process is slow, and trying to align a flat layer with a rolled layer is very difficult. The constant use of PEOX also limits adjustability of the layers once glued. Even the slightest misalignment can ruin the entire wrap and waste 
LTCC and silver paste. In addition to alignment, wrapping each layer one at a time (layer by layer) usually leads to a loose wrap, which as previously mentioned, will compound with each wrap until none of the remaining layers align correctly.

\section{Layer by Group}

The layer by group technique is the process of gluing multiple layers together flat and then wrapping the whole group in one or multiple rolls. Layers 1 to 4 are overlapped about halfway on top of each other, using the fiducial marks and tabs to align. Then all four layers are glued together using PEOX. A very minimal amount of PEOX should be applied on the surface near the edges of each layer. This layer 1 to 4 strip is shown in Figure 39. Once the PEOX dries, the layers create an almost continuous layer that can be rolled altogether at one time without gluing layer 1 to the jig. Layer 5 is then aligned using the fiducial marks and rolled on using the layer by layer technique previously discussed.

At this point the cathode structure is placed in the isostatic press where it is laminated at half the pressure $10.35 \mathrm{MPa}(1.5 \mathrm{ksi})$ at $70^{\circ} \mathrm{C}\left(158^{\circ} \mathrm{F}\right)$ for $10 \mathrm{~min}$. After lamination, layers 1 to 5 are securely bonded together which makes rolling layers 6 and 7 using the layer by layer technique very easy. Finally, the spacer strips are rolled on using PEOX, and the cathode structure is placed in the isostatic press to be laminated for a second time at half pressure. Laminating the cathode in stages, also known as progressive lamination, adds to the structural integrity of the cathode [77]. 


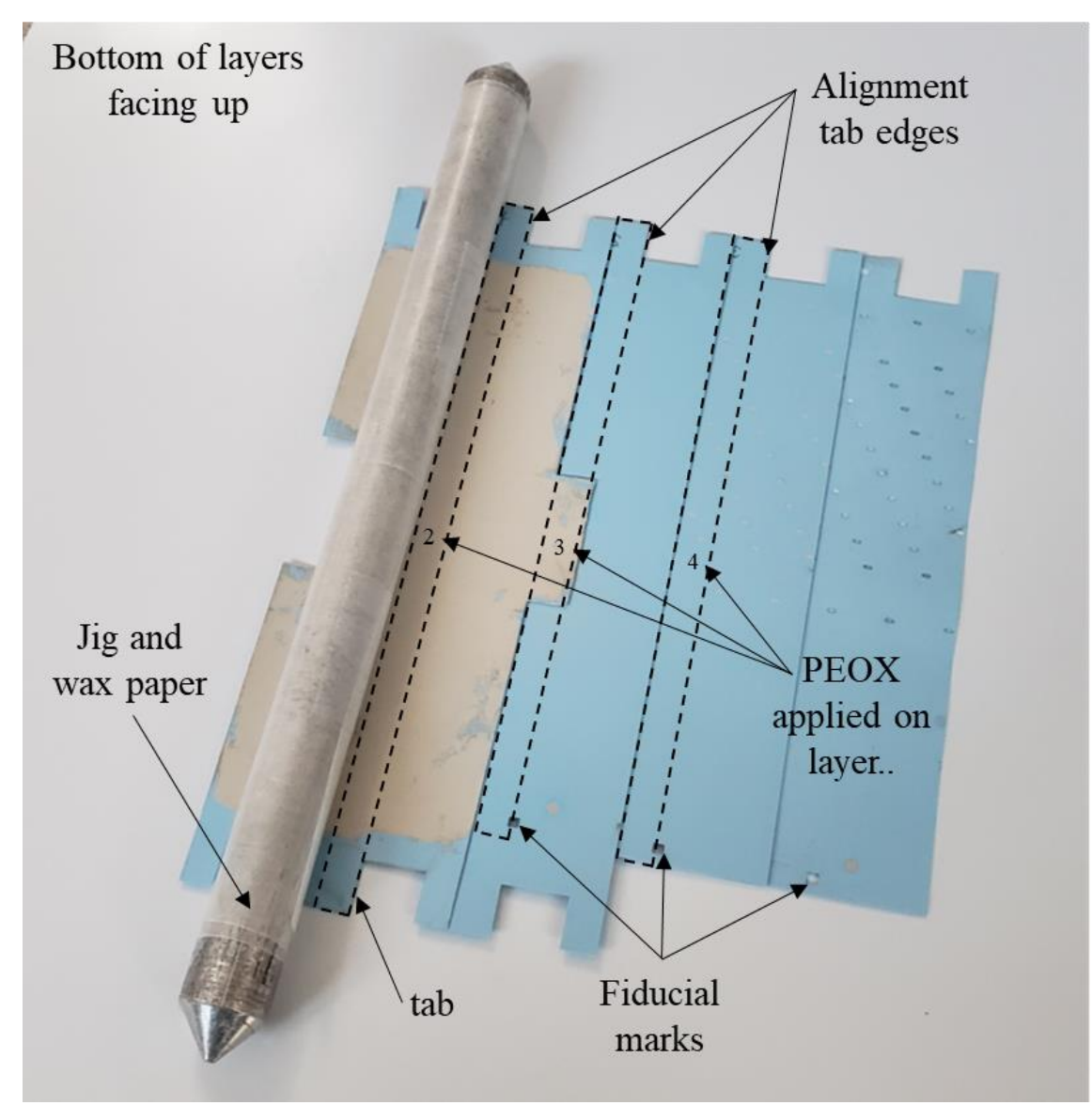

\section{Figure 39. Layer by group technique: layers 1 to 4 have been glued together with PEOX and are about to be wrapped around the jig}

Originally the layer by group technique consisted of gluing all seven layers together flat and then rolling, but after numerous wrapping attempts it was determined that layers 5 to 7 could not be wrapped using this technique. In order to get a tight wrap, the layers must be under tension while wrapping which tends to tear layers 5 to 7 . The way around this was to laminate in stages. The first lamination would compact layers 1 to 5 and shrink the diameter slightly. This shrinkage was enough to wrap layers 6 and 7 using the layer by layer technique with ease.

This layer by group technique is optimal for strong layers, such as layers 1 to 4 , because these layers can be bent and put under tension without tearing. If the layers are aligned and glued correctly, which is much easier while flat and with fiducial marks, then 
the rolled cathode structure will be wrapped tightly and correctly nearly every time. By using minimal amounts of PEOX, the jig can be rolled under pressure repeatedly until the seams of the layers meet. The concept is similar to tightening a roll of wrapping paper. The more the tube is rolled (like a rolling pin flattening dough), the more air bubbles or slack comes out between the layers. The same concept applies for the glued layers 1 to 4 around the cathode leading to a very tight wrap every time.

This technique is not optimal for sensitive layers, such as layers 5 to 7 , because rolling these layers multiple times causes them to tear or break apart. These layers cannot withstand the tension and bending that layers 1 to 4 can. This tearing issue is why this technique cannot be applied to sensitive layers, so layers 5 to 7 are wrapped using the layer by layer technique.

$\underline{\text { Results }}$

Since the success of each technique is user (operator) dependent, a subjective scoring system was chosen to compare the layer by layer technique to the layer by group technique for the new cathode LTCC wrapping process. The most heavily weighted score is repeatability. Being able to produce the same results every time not only saves material, time, and money, but also allows for both techniques to be accurately compared. This scoring will be based on two different outcomes: whichever technique is easier to align the layers and whichever technique is easier to achieve tight wraps. The second highest weighted score is the time required to master because of the consistent turnover in laboratory personnel. The technique that is easier to master would allow workers to start producing working cathode structures much quicker. The lowest weighted score was speed because these cathode structures are not being mass produced. Therefore, speed is 
important when comparing both techniques but not as critical for the overall optimization.

An objective scoring system was not chosen because there are too many uncontrollable operator variables when comparing between LTCC wrapping techniques. Some such variables are operator experience, meticulousness, and even motivation to produce a quality outcome. The repeatability, time required to master, and speed are all operator dependent which would require trials for numerous operators in order to objectively compare. This is why the scoring is subjective to how easy both techniques are and which are more likely to produce desired outcomes. The results of the scoring system are shown in Table 3.

Table 3. Subjective scoring of LTCC wrapping

\begin{tabular}{|l|c|c|c|}
\hline \multicolumn{1}{|c|}{ Parameters } & Weights & Layer by Layer & Layer by Group \\
\hline Alignment & 3 & 1 & 2 \\
\hline Wrap Tightness & 3 & 1 & 2 \\
\hline Mastery & 2 & 2 & 1 \\
\hline Speed & 1 & 1 & 2 \\
\hline & Total & 11 & 16 \\
\hline
\end{tabular}

The scored values in Table 3 were chosen from better technique (2) to worse technique (1). These scores where then scaled according (multiplied by) to the weights, and then summed to give the total score. The layer by group technique is more easily aligned because layers 1 to 4 are aligned flat as opposed to aligned rolled. The layer by group technique is wrapped tight more easily because it requires less wraps which reduces the possibility of errors; four wraps (layers 1 to 4 group, 5, 6, and 7) as opposed to seven wraps (layers 1, 2, 3, 4, 5, 6, and 7). The layer by layer technique requires less 
training because it only uses one wrapping technique (one layer at a time) as opposed to two (group roll and one layer at a time). The layer by group technique is faster because it wraps multiple layers at one time as opposed to one layer at a time. After summation, the layer by group had a larger total score which makes the layer by group technique the best and most optimized for the cathode manufacturing process. Figure 40a and Figure 40b shows examples of misaligned and aligned wraps respectively.

(a)

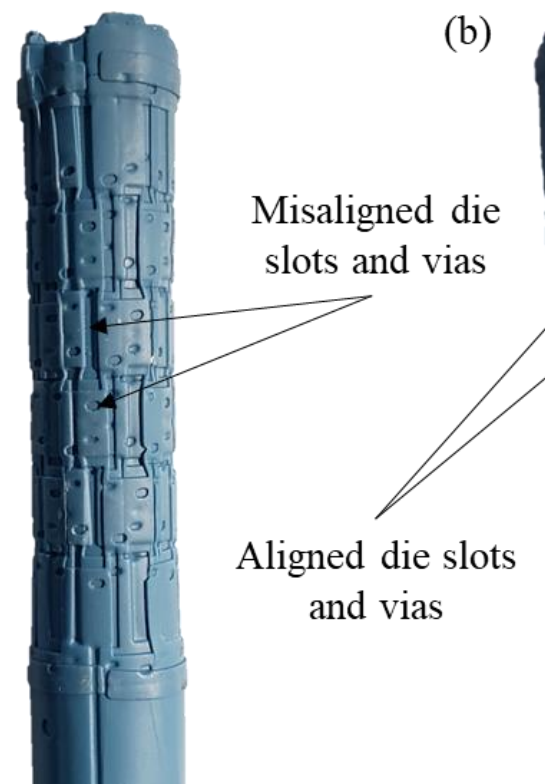

Figure 40. (a) Examples of not acceptable and (b) acceptable cathode wraps

\section{Via Fill}

Via filling techniques are implemented when connecting the internal transmission line (stripline) to the gated vacuum field emitters (die); this process was referenced earlier as the transmission line to die connection (tdc). As previously discussed, the tdc is composed of vias (holes) in layers 3 to 7 that when laminated create a tube-like structure that prevents leakage of silver paste throughout the tube. Once fired, this via tube and silver paste hardens to form the equivalent of an embedded electrically insulated wire. 
Issues arise; however, when one of the layers (layer 4 ground plane) has a silver paste layer that cannot electrically connect to the vias. In order to obtain the correct electrical characteristics of the stripline (layer 2), the stripline must be electrically insulated from both ground planes. Since the vias are connected to the stripline, they too must be electrically insulated from the ground planes.

Without the silver paste layer, both layers of LTCC would come into contact, closing the via tube in between layers. This concept is demonstrated between layers 7 to 6 shown in Figure 41. With the added thickness of the layer of silver paste, the LTCC layers do not come into contact. Depending on the via clearance hole diameter, the LTCC layers may or may not deform during lamination to close the gap. This concept is demonstrated between layers 5 to 4 shown in Figure 41 as well as proven later in Figure 46. The silver paste on layer 4 has a via clearance hole of a given diameter which will be discussed later in the clearance hole section and is shown in Figure 41.

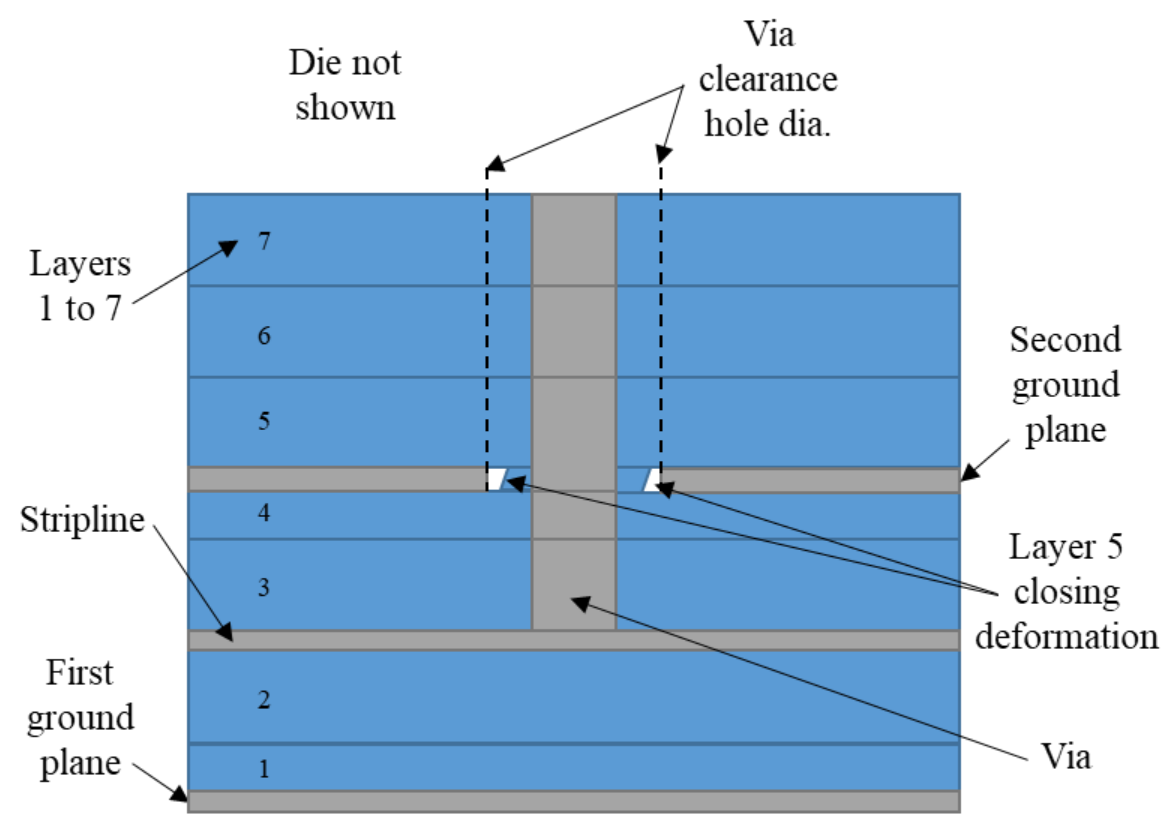

Figure 41. Cross section of the cathode structure showing both ground planes, the stripline, and one via clearance hole with a filled via 
Before manufacturing issues could be addressed, design challenges had to be overcome. Vias are traditionally circles of diameter $\sim 0.254 \mathrm{~mm}(0.01 \mathrm{in})$ that are cut out of each layer of LTCC using a punch as previously discussed. Since a punch was unavailable, a laser was used to cut the vias out of each layer. In order to circumvent the slow and tedious traditional laser drilling of vias (repeated pulses with the laser in one spot). The via diameters were increased to $1 \mathrm{~mm}(0.039 \mathrm{in})$ so that the laser could cut out the perimeter of the via rather than mill or drill out the entire via. This simplified and sped up the via cutting process in addition to making filling the via a lot easier which will be discussed later.

This larger diameter of via created a problem when connecting to the individual trace lines within the stripline on layer 2 . The preliminary version of the stripline had line widths of $\sim 130 \mu \mathrm{m}$ with spacing in between lines of $\sim 730 \mu \mathrm{m}$ which is not wide enough to accommodate the $1 \mathrm{~mm}$ diameter via. This was overcome by replacing the circular via with an oval via having a smaller diameter of $0.5 \mathrm{~mm}$ (0.020 in). This oval design was chosen in order to minimize the chance of electrically connecting two trace layers to one via (fitting within the $\sim 730 \mu \mathrm{m}$ gap), and to maximize the chance of contacting the specific trace line which is very small $(\sim 130 \mu \mathrm{m})$. The oval vias have a large diameter $1.130 \mathrm{~mm}(0.044 \mathrm{in})$ and small diameter $0.565 \mathrm{~mm}$ (0.022 in) which were designed to shrink to $\sim 1 \mathrm{~mm}$ (0.039 in) and $\sim 0.5 \mathrm{~mm}(0.020 \mathrm{in})$ respectively. Examples of a circular and oval via are shown in Figure 42a and Figure 42b, respectively. 


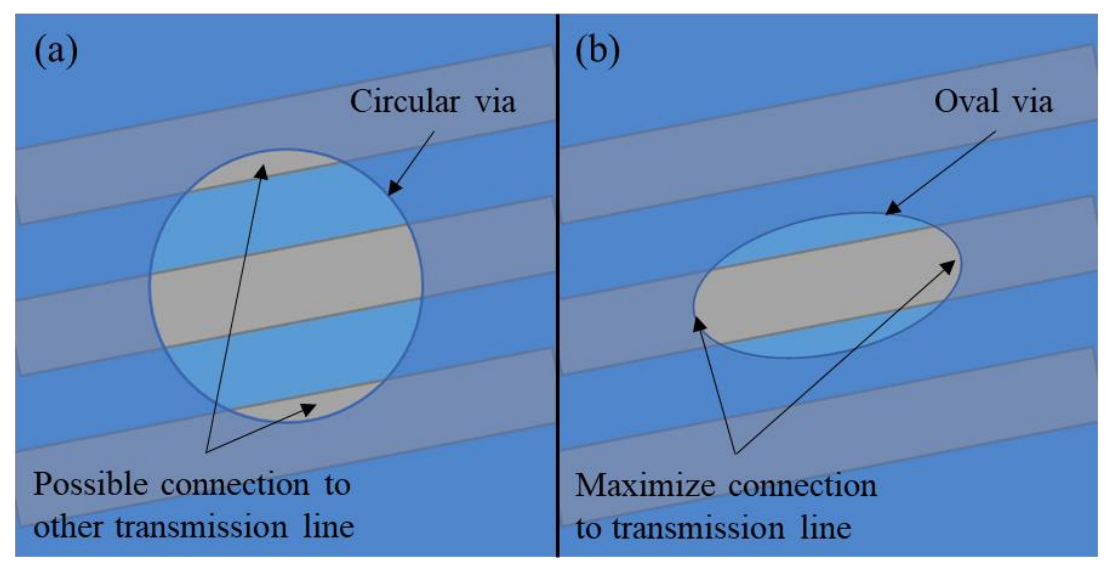

Figure 42. (a) Circular via and (b) oval via

As previously mentioned, another issue that arises is filling the vias on the cylindrical cathode structure which traditionally was accomplished using printers. These printers were unavailable; thus, for rapid prototyping alternative via filling techniques had to be explored. The techniques discussed below are different because the flat fill technique and the pneumatic pump technique fills the vias before and after wrapping the layers, respectively.

\section{Flat Fill}

The flat fill technique is to fill all of the vias one layer at a time in a flat layout before the layer is rolled. The LTCC sheets are shipped from DuPont on a white nonstick mylar paper that is required for this technique. A LTCC cathode structure layer is placed on top of the non-sticky side of the white mylar paper which should then be placed on a flat surface. Next, a via stencil is placed on top of the LTCC layer, shown in Figure 43. These stencils were made using the laser cutter to cut out only the via patterns for each layer; thus layers 3 to 7 have their own unique stencil. With the via stencil on top of the LTCC layer, the via holes are aligned. Once the via holes on the layer and stencil are aligned, silver paste is pushed across the stencil with a squeegee, pushing paste through the stencil into the vias on the LTCC layer. 


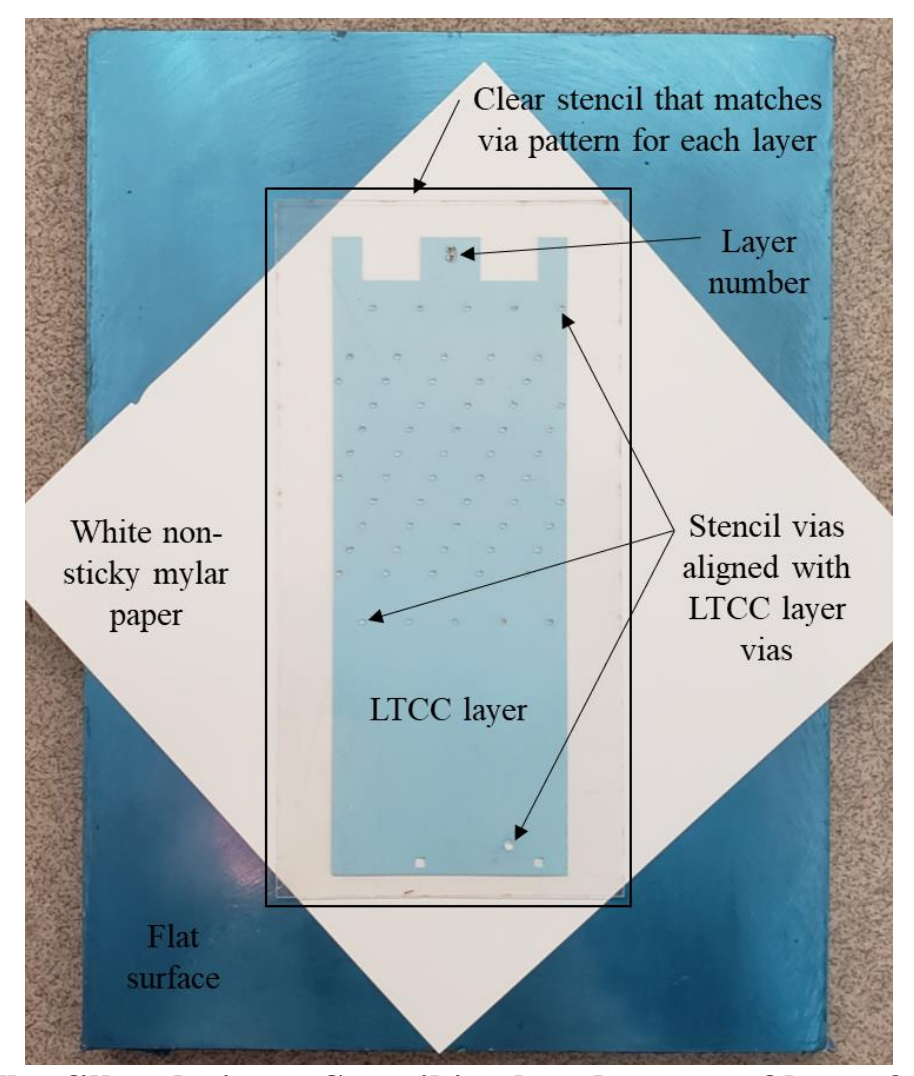

Figure 43. Flat fill technique: Stencil is placed on top of layer 3 which is placed on top of non-sticky mylar, then paste is squeegeed across the stencil

After all the vias are filled, the stencil is carefully removed by peeling the stencil off of the LTCC layer starting from the bottom (straight edge) and peeling towards the top (layer number edge). After the stencil has been removed, the LTCC layer plus mylar paper are placed into the heater (mylar paper down) to dry for a specific temperature and time. The temperatures and times specified in Table 4 correspond to the silver paste that was used to fill the vias. The times and temperatures for the Ferro 903-A [66], DuPont 6145 [50], and DuPont 6141 [51] were taken from literature and confirmed through testing. Testing consisted of filling the vias on a 10-mil thick LTCC layer (layer 3) and a 5-mil thick LTCC layer (layer 4) for all three silver pastes using the flat fill technique previously discussed. All three of the silver pastes dried completely, adhering to the 
inside of the vias for both LTCC layer thicknesses, confirming that the drying temperatures and times are correct.

Table 4. Drying temperatures and times for silver pastes

\begin{tabular}{|c|c|c|}
\hline Silver Paste (type of paste) & Temperature $\left({ }^{\circ} \mathrm{C},{ }^{\circ} \mathrm{F}\right)$ & Time (minutes) \\
\hline Ferro 903-A (conductor) & 70,158 & 30 \\
\hline DuPont 6145 (conductor) & 110,230 & 10 \\
\hline DuPont 6141 (via fill) & 110,230 & 5 \\
\hline
\end{tabular}

Any of the silver pastes can be used; however, DuPont 6141 works the best because it was specifically made to fill vias. After the silver paste has dried, the LTCC layer and mylar paper are removed from the heater and placed face down onto the flat surface (flat surface, LTCC layer, then mylar paper). Starting from a corner of the mylar paper, tightly roll the paper into a tube and continue to roll until the tube rolls off of the LTCC layer. Occasionally, a small amount of silver paste residue will be left on the mylar paper. Place the LTCC layer (side that was touching the mylar paper face up) back into the heater at the same temperature previously used for 5 minutes. Remove the LTCC layer from the heater and allow to cool for another 5 minutes before rolling or processing.

This technique is optimal for both types of LTCC layers (strong and weak) because it completely fills all of the vias without applying any force (tension, compression, or bending) to the layers. When the cathode structure is fired, the vias shrink which pushes the paste outward and causes slight bulges on the surface of the cathode structure. This creates slightly larger contact pads which are easy to connect to. In addition to the large contact pads, this paste expansion eliminates the need for paste in the layer 4 (5 mil) vias. The paste expansion will fill the vias on layer 4 , completing the tdc and decreasing the amount of paste and time used. By drying each layer prior to 
wrapping, the possibility of shorting (via contacting to the layer 4 ground plane) is greatly decreased because the dry paste cannot leak out of the via or spill over the edge of the via onto the surface of the LTCC layer. The only possibility of shorting now is due to wrapping layer misalignment which will be discussed later in the pneumatic pump section.

This technique's two downsides are both time issues. As shown above, the Ferro 903-A paste can take up to 30 minutes to dry. The DuPont 6141 paste; however, only takes 5 minutes to dry which drastically increases the efficiency of this technique. The other time issue arises during the wrapping process. If the layers are not wrapped correctly and the cathode fails or cannot be wrapped completely, all of the time spent filling the vias will have been wasted.

\section{Pneumatic Pump}

The pneumatic pump technique fills each of the via tube like structures, after the cathode has been wrapped and laminated. This technique can be applied to either the layer by layer or layer by group LTCC wrapping techniques. After wrapping layers 1 to 5 on the jig and laminating, the vias are filled using a pneumatic pump. The silver paste (any of the above mentioned) is loaded into a large syringe that is attached to the pump. The cathode (laminated layers 1 to 5) is placed horizontally on a 3-D printed stand, shown in Figure 44. Using an SRA-105 pneumatic pump, pressurized air is applied to the rear of the syringe which pushes paste out of a custom 200 um (0.008 in) diameter nozzle. The syringe is guided by hand to place the nozzle at the top (opening) of each via. The pump is activated by pressing a button that opens the pressure valve for approximately one second. This controlled expulsion of paste was used to increase 
efficiency. By adjusting the pressure applied, the entire via could be filled with only one push of the button.

The pressure for the Ferro 903-A/DuPont 6145 paste was $~ 0.21 \mathrm{MPa}$ (30 psi) and the pressure for the DuPont 6141 was $\sim 0.55 \mathrm{MPa}$ (80 psi). These pressure differences are due to the differences in paste viscosity. The Ferro 903-A and DuPont 6145 paste has a viscosity of $\sim 200$ and $\sim 160 \mathrm{~Pa}^{*} \mathrm{~S}$, respectively while the DuPont 6141 has a viscosity of $\sim 2100 \mathrm{~Pa}^{*} \mathrm{~S}$. Since the pressure could be easily adjusted with the turn of a knob, these pressures were determined with a trial and error approach. After all 60 of the vias are filled, the cathode is placed in the heater at $\sim 70^{\circ} \mathrm{C}\left(158^{\circ} \mathrm{F}\right)$ for $10 \mathrm{~min}$, regardless of the paste used. This dries the paste enough for layers 6 and 7 to be wrapped and for the whole cathode to be laminated for the last time.

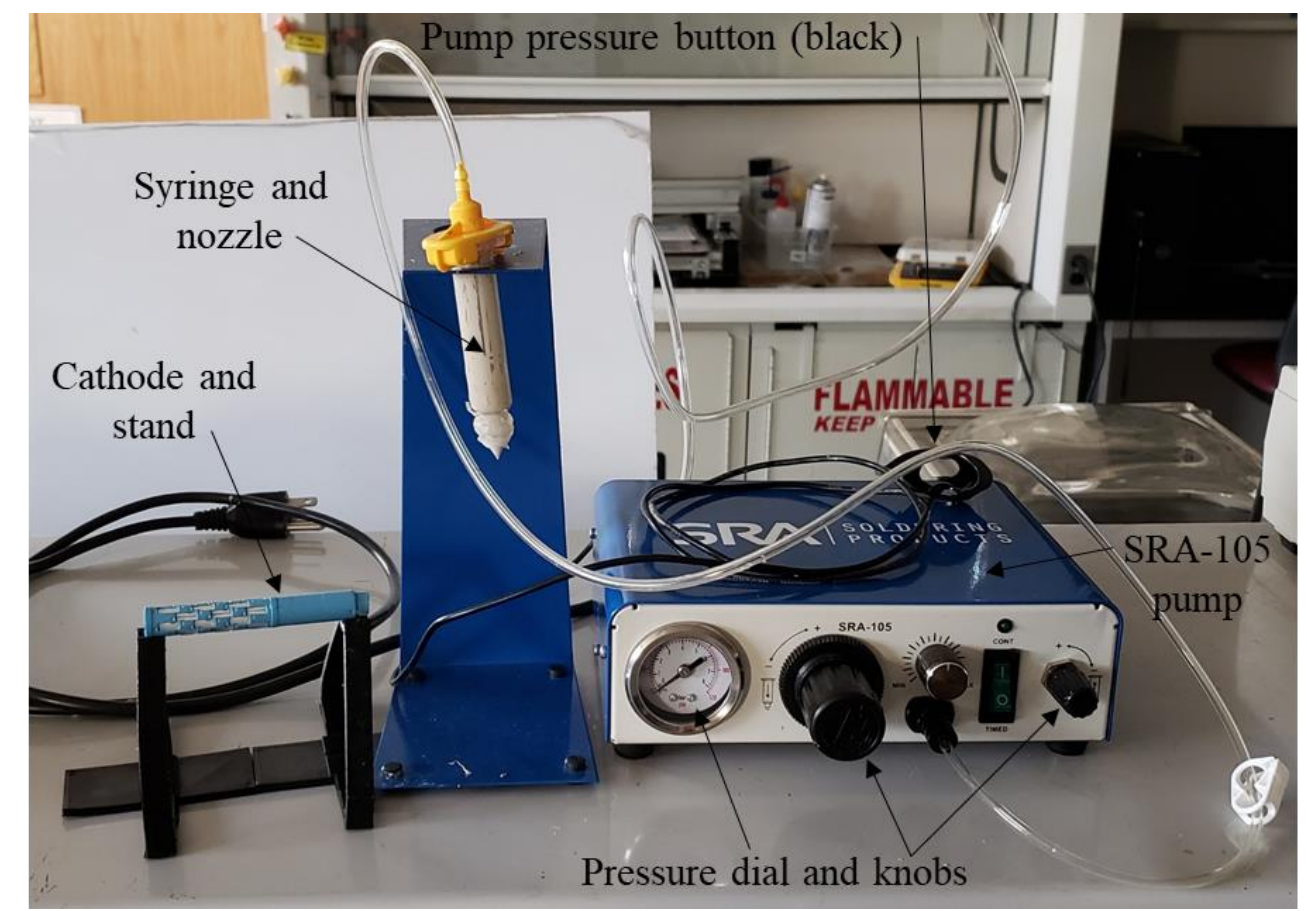

Figure 44. Pneumatic pump technique: pressure is set, the nozzle is lined up with the vias on the cathode, then the button is pressed to push paste out of the nozzle 
After the final lamination, some of the vias may not be completely filled and may need to be filled again before or after firing. The second filling of vias, pre-fire, may cause the surface of the via to be messy and occasionally cause shorting due to the close proximity of the second (top of layer 4) ground plane. The second filling of vias, postfire, will allow for easy cleaning of overfilled vias but will also require a second firing. The second firing does not change the cathode geometry or any of the characteristics of the cathode structure.

Originally this technique was designed to completely wrap the cathode, layers 1 to 7 without laminating, and then fill the vias. The first attempts shorted because the paste would leak out between the layers. This leakage was due to the paste not being dry as well as the cathode not being laminated. The next attempt was to laminate the cathode after wrapping layers 1 to 7 and then fill the vias. This attempt also failed because the vias collapse inward and closed during lamination if not filled. This collapse is due to the vias not changing size as the layer size increases (layer 3 to 7). The vias do not change size because the electrically insulated wire that the via tube is supposed to replicate, has to maintain a constant diameter. If the diameter changes, then the electrical properties will change as well. The next attempt occurred while finalizing the layer by group technique. It was observed that the vias up to layer 5 after lamination, only collapse slightly. They do not completely close, which lead to filling the vias after lamination of layers 1 to 5 and then ultimately to the finalized process for the pneumatic pump technique.

This technique is optimal in regards to speed for two reasons. The first is that filling the whole via at one time is much more efficient than individual layers of each via. 
The second, is if the cathode is not wrapped correctly or fails while being wrapped. The time spent filling the vias (flat fill technique) and the silver paste is not wasted. In addition to speed, by not filling the vias prior to wrapping, the vias can be used to align the layers more easily. Instead of an alignment tab on the top and a fiducial mark on the bottom, this approach creates five to ten potential fiducial marks along each layer edge and 60 fiducial marks once wrapped. The lack of filled vias can even be used to visually check connection to the stripline on layer 2 . The stripline can be followed to make sure only one trace line connects to each via tube.

This technique is not optimal for obtaining fully filled vias. Because of the viscosity of the paste and the slight misalignment of the vias that comes with human variability, the vias may not fill completely or even reach the transmission line. This technique relies primarily on aligned vias and any misalignment can cause the vias to not fill correctly as shown in Figure 45b. Figure 45a show how the completely filled vias using the flat fill technique can combat this misalignment issue. In addition to via alignment, if the via is over filled with the pump, then the excess paste can drip over the surface of layer 7. This drip could connect to the other via or even drip all the way down the die support section onto layer 4 ground plane, shorting out the entire via system.
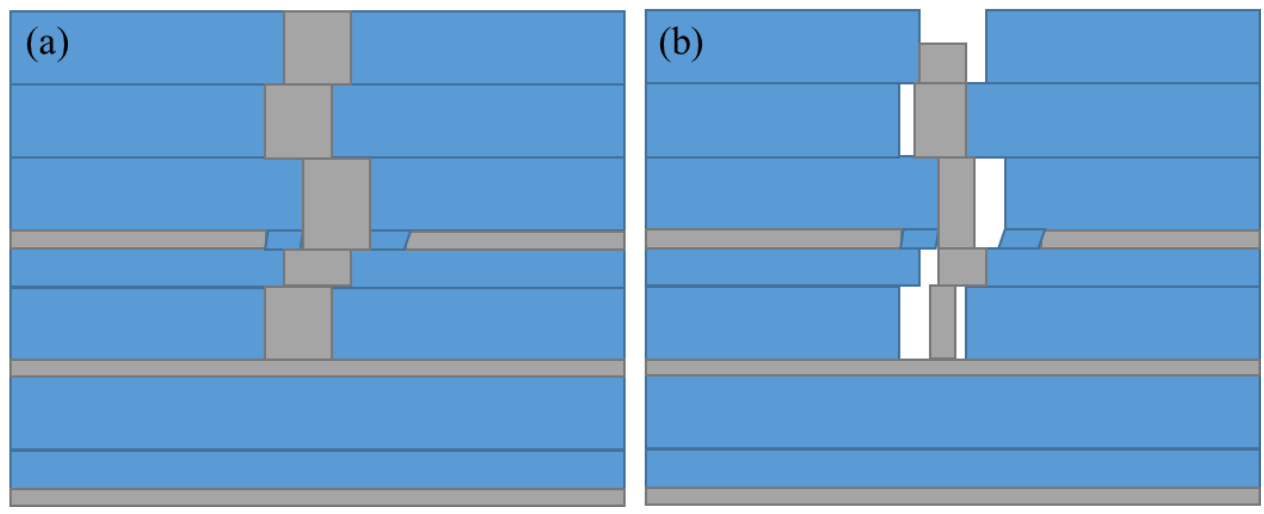

Figure 45. (a) Example of fully filled and (b) not fully filled vias 


\section{$\underline{\text { Clearance Hole }}$}

The cathode transmission line (stripline) requires two ground planes (layer 1 and 4) that enclose the stripline (layer 2). The vias connect from the stripline (layer 2) to the die (layer 7) which means the vias pass through the outer ground plane. This connection requires holes in the ground plane that will maximize the ground plane surface area but not short to the vias. As previously discussed, layer 5 must deform to close the gap in the via tube and prevent any electrical connection from occurring. A test was designed to determine the minimum diameter of the clearance holes in the ground layer. Four different OD clearance holes were chosen: $1.5 \mathrm{~mm}$ (0.06 in), $2 \mathrm{~mm}(0.08 \mathrm{in}), 2.5 \mathrm{~mm}$ (0.10 in), $3 \mathrm{~mm}(0.12 \mathrm{in})$, and each OD was sectioned to verify via tube closure. The via clearance hole test for all of the outer diameters (OD) is shown in Figure 46.

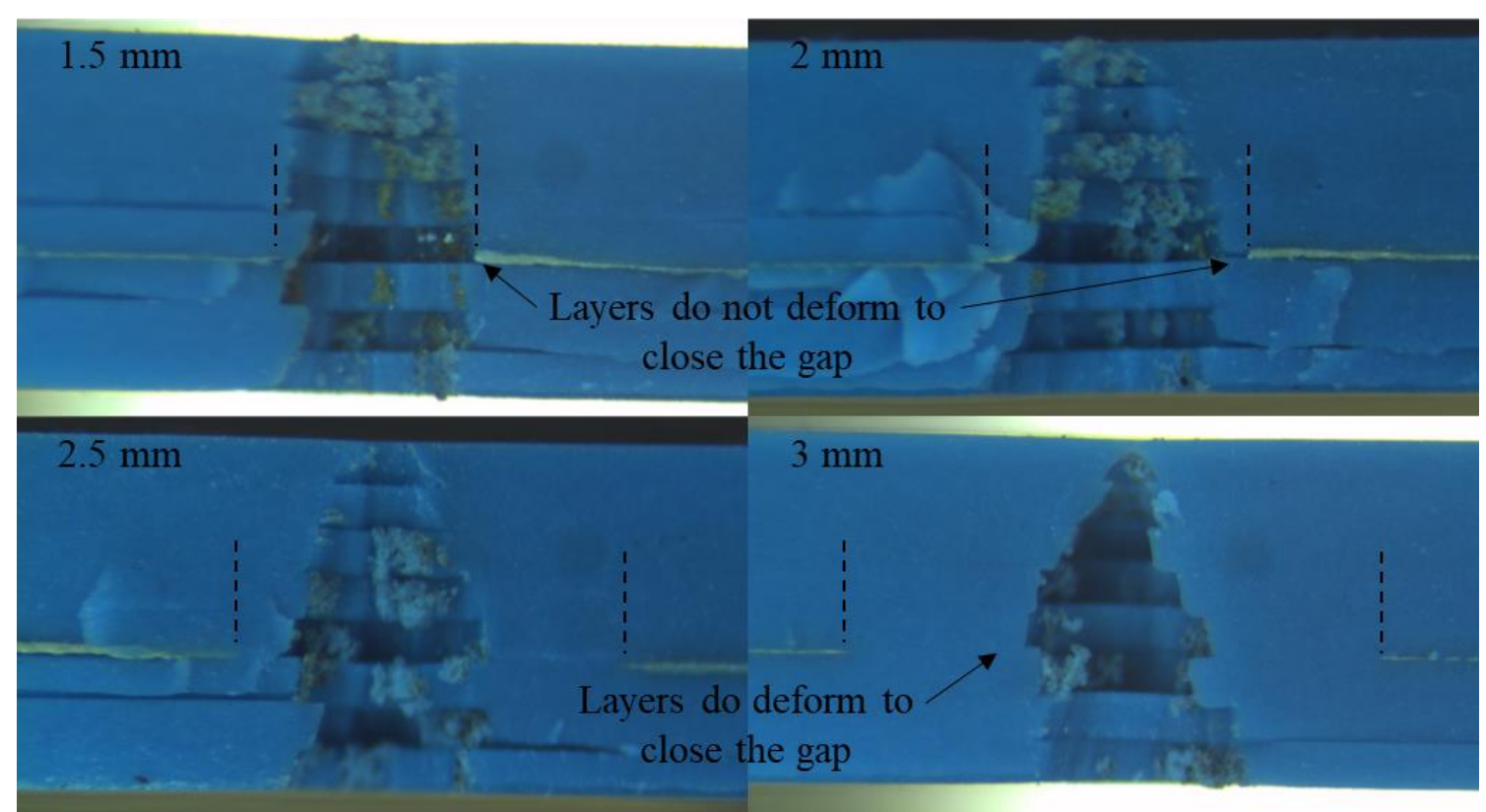

Figure 46. Via clearance hole test cross sections

As shown in Figure 46, the via columns did not close for OD $1.5 \mathrm{~mm}$ and $2 \mathrm{~mm}$.

OD $2.5 \mathrm{~mm}$ was questionable due to alignment, and the $3 \mathrm{~mm}$ hole was clearly closed. 
An additional test was used to verify these results. Five via columns were assembled and filled, through ground holes of OD $2.5 \mathrm{~mm}$ and $3 \mathrm{~mm}$, and alignment was verified using alignment pins. The results were that all five via columns closed for both sets and did not short to the ground plane through the OD holes. Thus, verifying that the minimum OD for clearance holes is $2.5 \mathrm{~mm}$ for vias $\sim 1 \mathrm{~mm}$. Since this test was flat and alignment for the wrapped cathode is more difficult, a via clearance hole OD was chosen as $3 \mathrm{~mm}$ for this cathode structure. By increasing the clearance holes on layer 4, the ground plane coverage decreases which may affect the stripline electrical characteristics which requires a top and bottom ground plane.

$\underline{\text { Results }}$

Because the success of each technique is user (operator) dependent, a subjective scoring system was chosen to compare the flat fill technique to the pneumatic pump technique for the new cathode via filling process. The most heavily weighted score is repeatability. Being able to produce the same results every time not only saves material, time, and money, but also allows for both techniques to be accurately compared. This scoring will be based on two different outcomes: whichever technique is less likely to short electrically to the ground on layer 4 and whichever technique is the most likely to connect to the transmission line on layer 2. The second highest weighted score is the time required to master because of the consistent turnover in laboratory personnel. The technique that is easier to master would allow workers to start producing working cathode structures more quickly. The lowest weighted score was speed because these cathode structures are not being mass produced. Therefore, speed is important when comparing both techniques but not as critical for the overall optimization. 
An objective scoring system was not chosen because there are too many uncontrollable operator variables when comparing between via filling techniques. Some such variables are operator experience, meticulousness, and even motivation to produce a quality outcome. The repeatability, time required to master, and speed are all operator dependent which would require trials for numerous operators in order to objectively compare. This is why the scoring is subjective to how easy both techniques are and which are more likely to produce desired outcomes. The results of the scoring system are shown in Table 5.

Table 5. Subjective scoring of via filling

\begin{tabular}{|l|c|c|c|}
\hline \multicolumn{1}{|c|}{ Parameters } & Weights & Flat Fill & Pneumatic Pump \\
\hline Electrical Shorting & 3 & 2 & 1 \\
\hline Trans. to Die Connect & 3 & 2 & 2 \\
\hline Mastery & 2 & 1 & 2 \\
\hline Speed & 1 & 1 & 12 \\
\hline
\end{tabular}

The scored values in Table 5 were chosen from better technique (2) to worse technique (1). These scores where then scaled according (multiplied by) to the weights, and then summed to give the total score. The flat fill technique is less likely to short because the only possibility is due to layer misalignment as opposed to overflow or leakage. The flat fill technique is more likely to fill the vias completely which significantly increases the chances to connect the transmission line to the die (layers 3 to 7). The pneumatic pump technique requires less training because it consists of placing the nozzle in the via and pushing a button as opposed to aligning a template and then squeegeeing paste across. The pneumatic pump technique is faster because it fills the 
complete vias at one time as opposed to each layer of the vias and then drying times.

After summation, the flat fill had a larger total score which makes the flat fill technique the best and most optimized for the cathode structure manufacturing process. Figure $47 \mathrm{a}$ and Figure $47 \mathrm{~b}$ shows examples of incompletely and completely filled vias respectively.

(a)

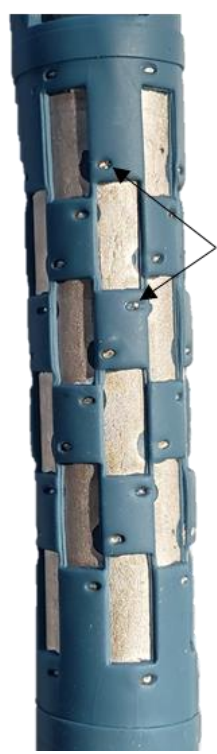

Incompletely filled vias

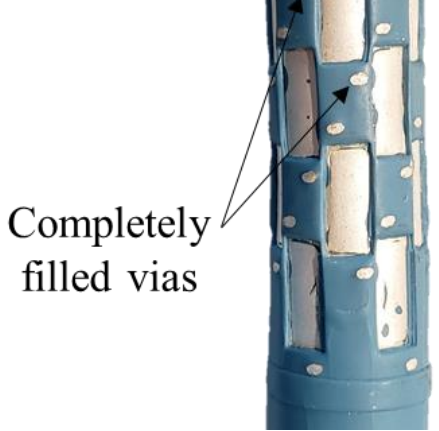

Figure 47. (a) Examples of bad and (b) good via filling

\section{Facet Plates}

As previously mentioned, the facet plates act as electron hop funnels that protect the die and increase uniformity of the electron emission source. The cathode design has three die aligned vertically with two emission sources each, which means the facet plates must have six funnel slits per facet plate. Each funnel must be longer $(>4.8 \mathrm{~mm})$ and wider $(>1.1 \mathrm{~mm})$ than the emission area of the die, and there must be a pointed edge inbetween the two funnels of each die to prevent electrons from charging the dielectric surface. The 10-sided cathode structure determined the facet plate edges should be $\sim 36^{\circ}$. The facet plate thickness is $\sim 1.2 \mathrm{~mm}$, and the emission slits should be as small as possible $\sim 300 \mu \mathrm{m}$. Lastly, the width of the wider side of the facet plates should be $\sim 4.4 \mathrm{~mm}$. A 
cross sectional view of the facet plate design is shown in Figure 48 where the three aligned die are oriented out of the page.

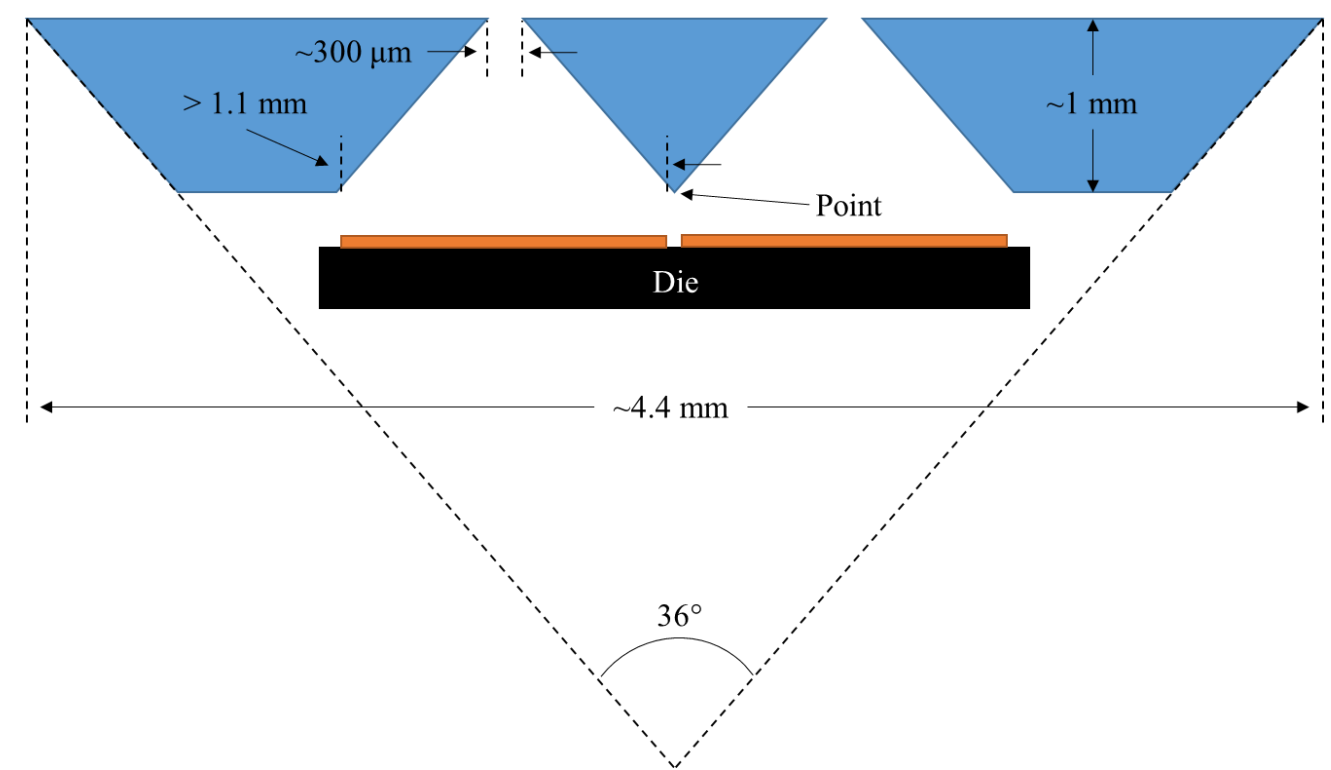

Figure 48. Diagram of facet plate (blue) fired geometry

The technique for building the facet plates is to remove material in a ' $V$ ' shape in order to produce the correct geometry. Both techniques require the same initial starting block of LTCC which starts by cutting five rectangles with area $76.2 \mathrm{~mm}$ by $127 \mathrm{~mm}$ (3 in by 5 in) of 10-mil thick LTCC. Then the five rectangles are laminated together under 20.7 $\mathrm{MPa}(3 \mathrm{ksi})$ at $70^{\circ} \mathrm{C}\left(158^{\circ} \mathrm{F}\right)$ for $10 \mathrm{~min}$. This will give a block of LTCC that has enough area to cut out all ten facet plates with a thickness $(\sim 1.2 \mathrm{~mm})$ that will shrink once fired to the desired $1 \mathrm{~mm}$. The laser technique uses a laser to burn or weaken the material in the slots which is removed my hand later. The mill technique uses a mill with a ' $\mathrm{V}$ ' shaped end mill (tool) to remove the material in the slots.

\section{Laser Technique}

The laser technique uses an M-300 Universal laser to cut the desired geometry of the facet plates. The LTCC block, previously discussed, is placed into the laser setup 
where the facet plate outline is cut twice. Both cuts are applied to the slots and outline of the facet plates to achieve the ' $\mathrm{V}$ ' shaped slots and angled edges of $\sim 36^{\circ}$. The cutting parameters are shown in Table 6 where PPI is pulse per inch. The Z-height offset number (inches) is in reference to the current single layer Z-height. The current single layer Zheight is the laser height that is used to cut one sheet (10-mil or 5-mil thick) of LTCC. The Z-height offset number should be added to the current single layer Z-height for both of the cuts. For example, if the Z-height for cutting one layer (normal operation) is 4.16, then the cut $1, \mathrm{Z}$-height would be $4.16+(-0.02)$ which would equal 4.14 .

Table 6. Laser cutting parameters

\begin{tabular}{|c|c|c|c|c|}
\hline Cut Number & $\begin{array}{c}\text { Z-height } \\
\text { Offset (in) }\end{array}$ & $\%$ Power & \% Speed & PPI \\
\hline 1 & -0.02 & 85 & 0.8 & 70 \\
\hline 2 & -0.11 & 85 & 0.8 & 40 \\
\hline
\end{tabular}

The two cuts are used to weaken different portions of the LTCC material to achieve the desired ' $V$ ' shape slots. Lasers work by focusing the beam at a single point (focal point). Normal (single sheet) LTCC cutting operations cut at this focal point to give close to vertical edges (perpendicular to cutting bed). By raising and lowering the laser height (Z-height), the vertical cut becomes a slanted cut, in theory, shown in Figure 49. In actuality, changing the Z-height burns and weakens different portions of the block of LTCC along this slanted line. The first cut lowers the material, raising the focal point to burn the middle portion of the slot, weakening most of the material vertically. The second cut raises the focal point more to burn a larger portion of the top of the slot. Figure 50 shows the actual laser technique cutting out the outlines of the facet plates. 
(a)

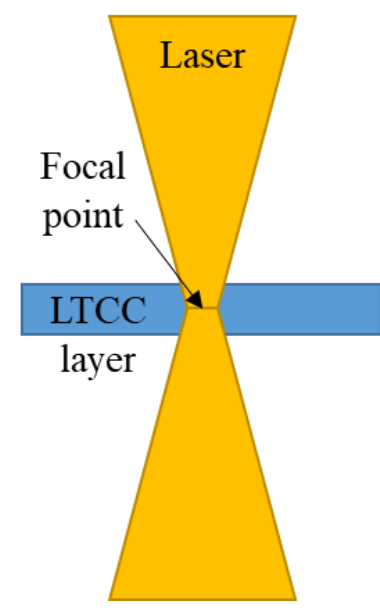

Figure 49. (a) Normal Z-height, (b) cut 1, and (c) cut 2 (b)

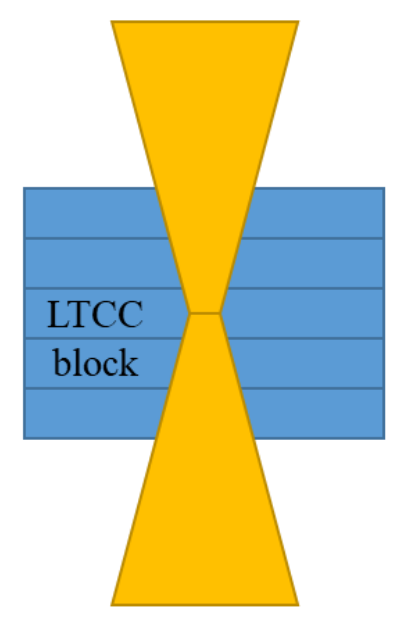

(c)

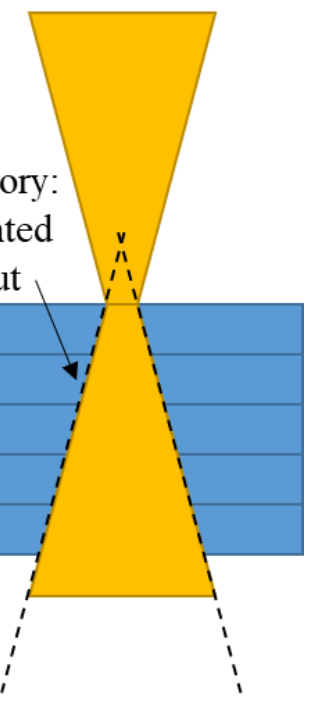

After the second cut, the facet plates are removed from the bed and the burned portions of the slots are removed by carefully scraping them out. This scraping is usually done by hand using a drill bit or end mill engraving tool. At this point, the laser has burned out material in the desired ' $\mathrm{V}$ ' shape, but additional scraping is usually required in order to achieve the pointed edge between the two slots. Visually inspect the facet plates to verify that there is a pointed edge between each of the two slots. There should be three sets of two slots for a total of six slots and three pointed edges. After verification of the pointed edges, the facet plates are fired in the furnace under normal conditions. Figure 50 shows the LTCC block with the facet plates being cut out using the laser technique. 


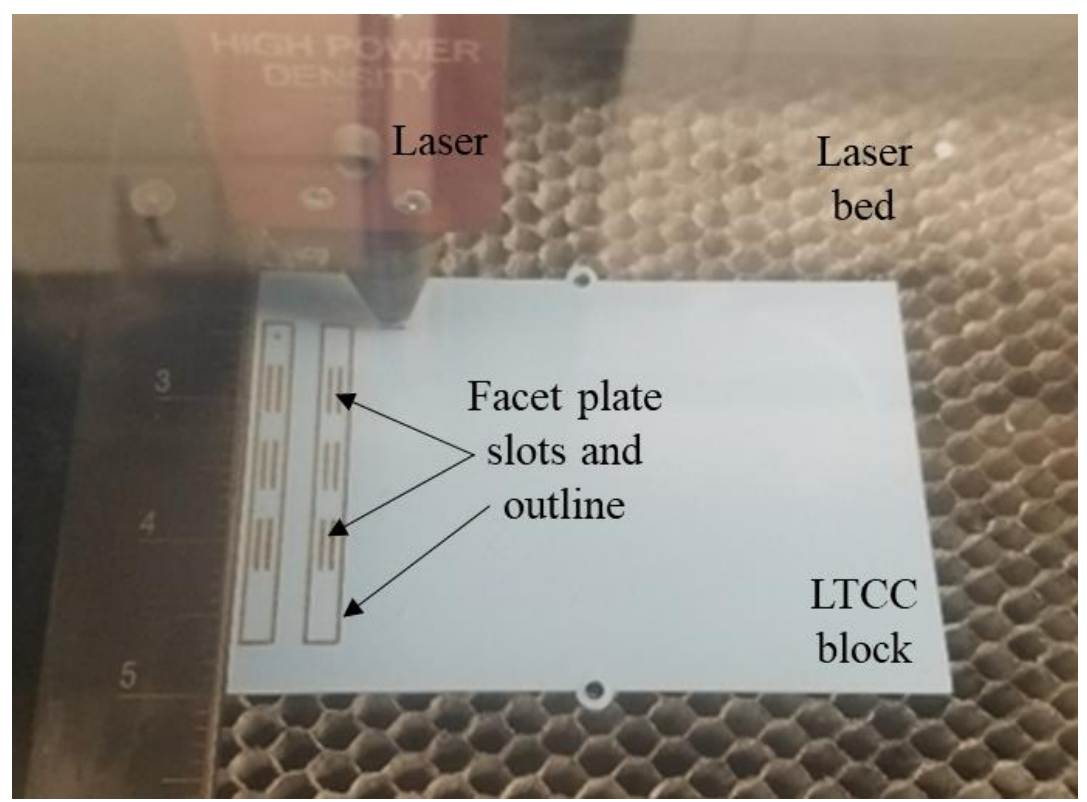

Figure 50. Laser technique: LTCC block is placed on the bed, then the laser burns the facet plate slots and outlines

This technique is optimal for cutting single layers because the laser cuts quickly and precisely all the way through the layer. This technique is not optimal for multiple layers because the laser burns the material rather than removing it (ablation). Offsetting the focal point to achieve the ' $\mathrm{V}$ ' shape works because the burned material does remove easily and resembles the ' $\mathrm{V}$ ' shape; however, the moving of the laser to create the slots causes differences in burn intensities along the slots. These differences in burn intensities result in sections of large burned material and small burned material which, when removed, causes very jagged edges along the slots. Essentially, this causes differing amounts of burned material to be removed which causes variable (not consistent) widths along the slots. The last step of hand scraping causes all of the slots to be different as well.

\section{$\underline{\text { Mill Technique }}$}

The mill technique uses a Bungard PCB mill to cut the desired geometry of the facet plates. The LTCC block, previously discussed, is taped (scotch tape) to a millable 
substrate (usually clear plastic) and placed on the vacuum chuck located on the mill bed and secured using the vacuum. If the substrate is not secured fully with the vacuum alone, tape may be added to insure the substrate stays fixed. Like the laser technique, this mill technique also requires two cuts. The first cut is using the $60^{\circ}$ ' $\mathrm{V}$ ' tip end mill to cut out the ' $\mathrm{V}$ ' shaped slots in the facet plates. The second cut is using the $40^{\circ}$ ' $\mathrm{V}$ ' tip end mill to cut out the outline of the facet plates.

Insert the $60^{\circ}$ end mill into the collet and set the offset to cut the facet plate cutting profiles on the thin block of LTCC. The offset is used to move the cutting profile to the desired location on the material to be cut (in this case, the thin block of LTCC). This offset (const.) will stay the same for both cuts to insure proper alignment between cuts. After the offset is set, set the board thickness to where the end mill just barely scrapes the surface of the LTCC block. Record this dimension (zero1). Now subtract 0.2 $\mathrm{mm}$ from zero1 (subtracting makes the cut deeper) to achieve the $\sim 300 \mu \mathrm{m}$ bottom side slot width. The iterations number determines how many passes will be used to achieve the depth measurement. This number should remain high (obtaining $\sim 0.5 \mathrm{~mm} / \mathrm{pass}$ cutting) to prevent breaking the thin strip between the slots. Leave the base settings the same and run the cut for the slots using the parameters shown in Table 7 for cut 1 . Figure 51 shows the depth of the angled end mills for each cut during the mill technique. 


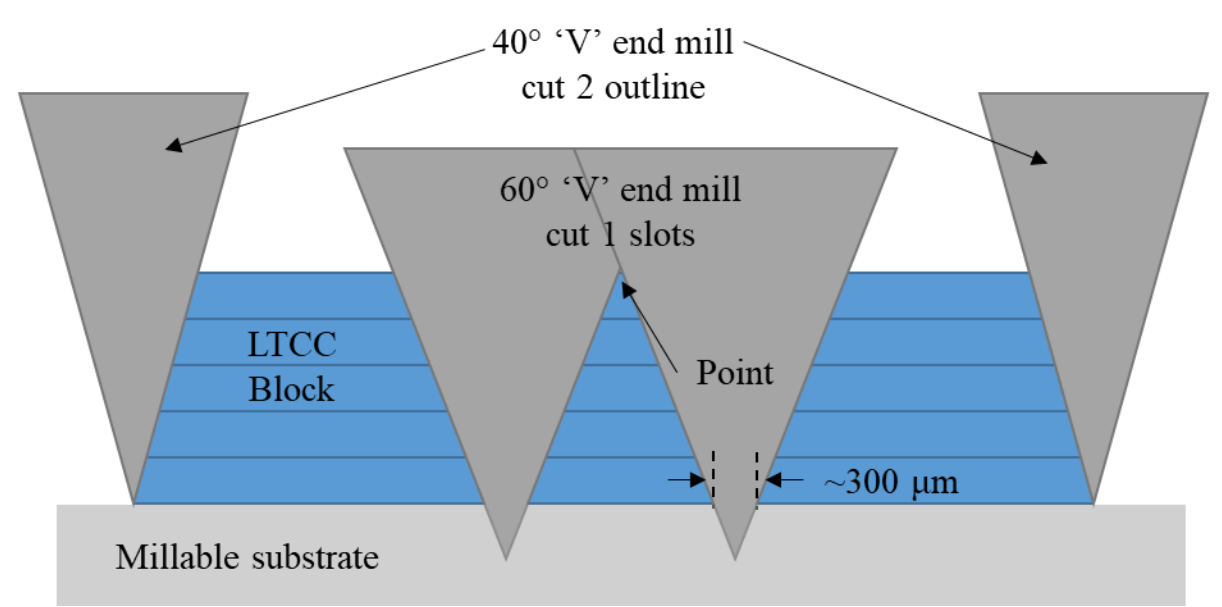

Figure 51. Mill cutting cross sectional diagram

After cut 1 , verify that the slots are all the way through the block of LTCC and that a pointed edge exists between each slot. Replace the $60^{\circ}$ end mill with the $40^{\circ}$ ' $\mathrm{V}$ ' tip end mill and set the board thickness to where the end mill just barely scrapes the surface of the LTCC block. Record this new dimension (zero2). Change the routing file to cut the outline of the facet plates and run again using the parameters shown in Table 7 for cut 2 . After this second pass, remove any leftover chips of LTCC and visually inspect the facet plates to verify that there is a pointed edge between the two slots for all three sets. The facet plates are now ready to be fired in the furnace under normal conditions.

\section{Table 7. Mill technique cutting parameters}

\begin{tabular}{|c|c|c|c|c|}
\hline \multicolumn{5}{|c|}{ Tool parameters for both passes } \\
\hline Depth (mm) & Iterations & X/Z - feed (mm/min) & \multicolumn{2}{c|}{ Spindle (1/min) } \\
\hline 1.2 & 24 & 250 & \multicolumn{2}{c|}{40,000} \\
\hline \multicolumn{5}{|c|}{ Main menu parameters for each pass } \\
\hline Cut Pass & Offset (mil) & Board (mm) & End Mill $\left({ }^{\circ}\right)$ & Route file \\
\hline 1 & const. & Zero1 - 0.2 & 60 & slots \\
\hline 2 & const. & Zero2 & 40 & outline \\
\hline
\end{tabular}




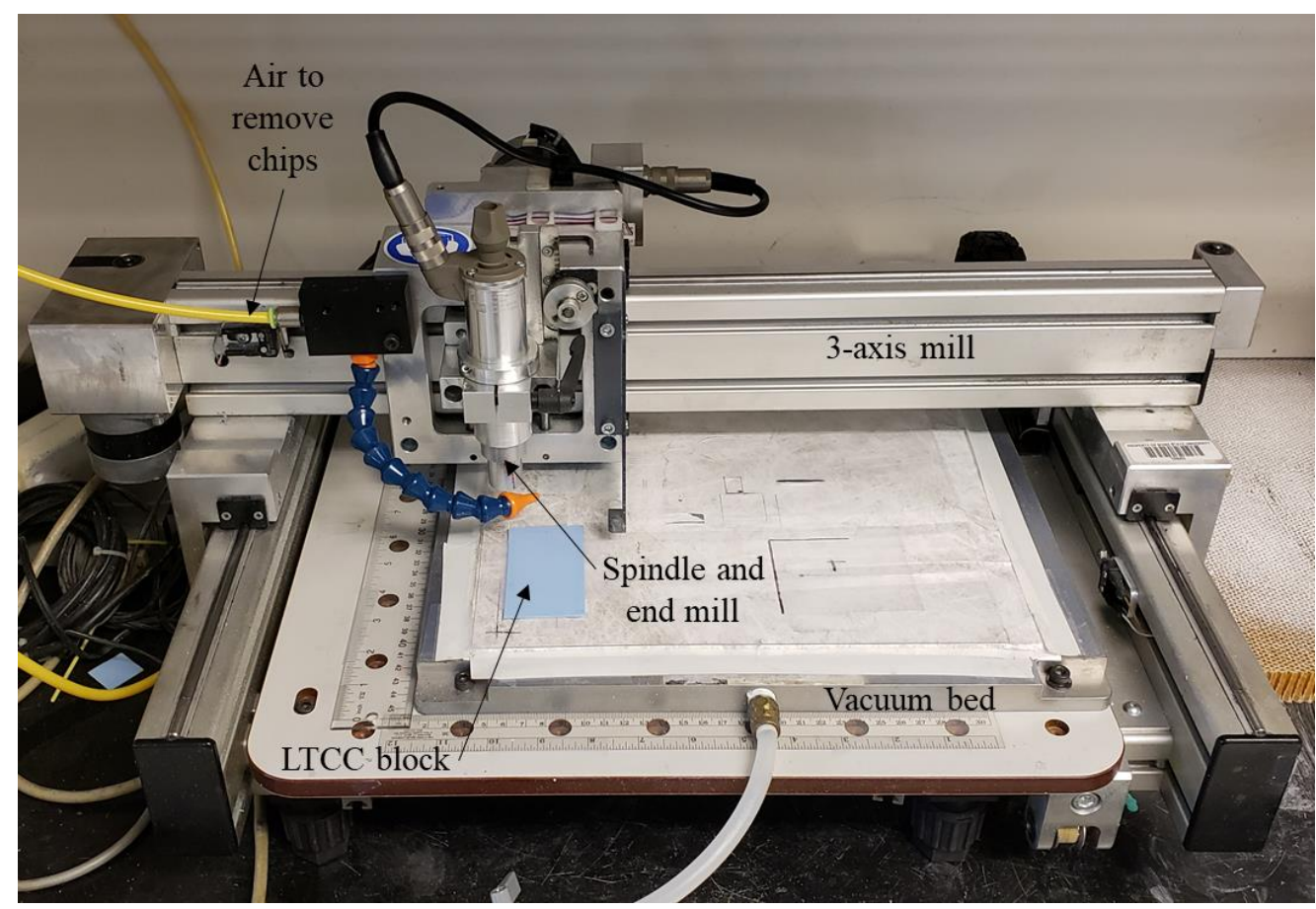

Figure 52. Mill technique: LTCC block is taped to the bed, then the $60^{\circ}$ and $40^{\circ}$ endmills cut the slots and outlines of the facet plates, respectively

This technique is optimal for cutting multiple layers because the mill cleanly and quickly removes the material for any layer thickness. This technique is also extremely repeatable because of the automation from the computer controlled 3 -axis mill. The end mills also leave smooth surface finishes as well as very accurate ' $\mathrm{V}$ ' shape slots which is beneficial for uniform electron emission. This technique is not optimal for speed because the milling process is very slow and the tool changing also adds time to the overall process.

$\underline{\text { Results }}$

Because the success of each technique is user (operator) dependent, a subjective scoring system was chosen to compare the laser technique to the mill technique for the new cathode facet plate process. The most heavily weighted score is repeatability. Being able to produce the same results every time not only saves material, time, and money, but 
also allows for both techniques to be accurately compared. This scoring will be based on two different outcomes: whichever technique is more repeatable and whichever technique can produce smooth ' $\mathrm{V}$ ' shaped slots. The second highest weighted score is the time required to master because of the consistent turnover in laboratory personnel. The technique that is easier to master would allow workers to start producing working cathode structures more quickly. The lowest weighted score was speed because these cathode structures are not being mass produced. Therefore, speed is important when comparing both techniques but not as critical for the overall optimization.

An objective scoring system was not chosen because there are too many uncontrollable operator variables when comparing between facet plate techniques. Some such variables are operator experience, meticulousness, and even motivation to produce a quality outcome. The repeatability (for laser technique), time required to master, and speed (including learning software and setup for mill) are all operator dependent which would require trials for numerous operators in order to objectively compare. This is why the scoring is subjective to how easy both techniques are and which are more likely to produce desired outcomes. The results of the scoring system are shown in Table 8.

Table 8. Subjective scoring of facet plates

\begin{tabular}{|l|c|c|c|}
\hline \multicolumn{1}{|c|}{ Parameters } & Weights & Laser & Mill \\
\hline Repeatability & 3 & 1 & 2 \\
\hline Smooth 'V' Slots & 3 & 1 & 1 \\
\hline Mastery & 2 & 2 & 1 \\
\hline Speed & 1 & 2 & 15 \\
\hline & Total & 12 & 2 \\
\hline
\end{tabular}


The scored values in Table 8 were chosen from better technique (2) to worse technique (1). These scores were then scaled according (multiplied by) to the weights and then summed to give the total score. The mill technique is more repeatable because it is a mechanically automatic technique as opposed to a human hand technique. The mill techniques can clearly (visual inspection) produce smoother ' $\mathrm{V}$ ' shaped slots. The laser technique requires less training because it consists of using the laser cutter and a hand as opposed to setting up and learning the mill software. The laser technique is faster because it cuts and scrapes (by hand) faster than the mill can remove the material. After summation, the mill had a larger total score which makes the mill technique the best and most optimized for the cathode manufacturing process. Figure 53a, Figure 53c and Figure 53b, Figure 53d shows examples of facet plates using the laser and mill techniques, respectively.

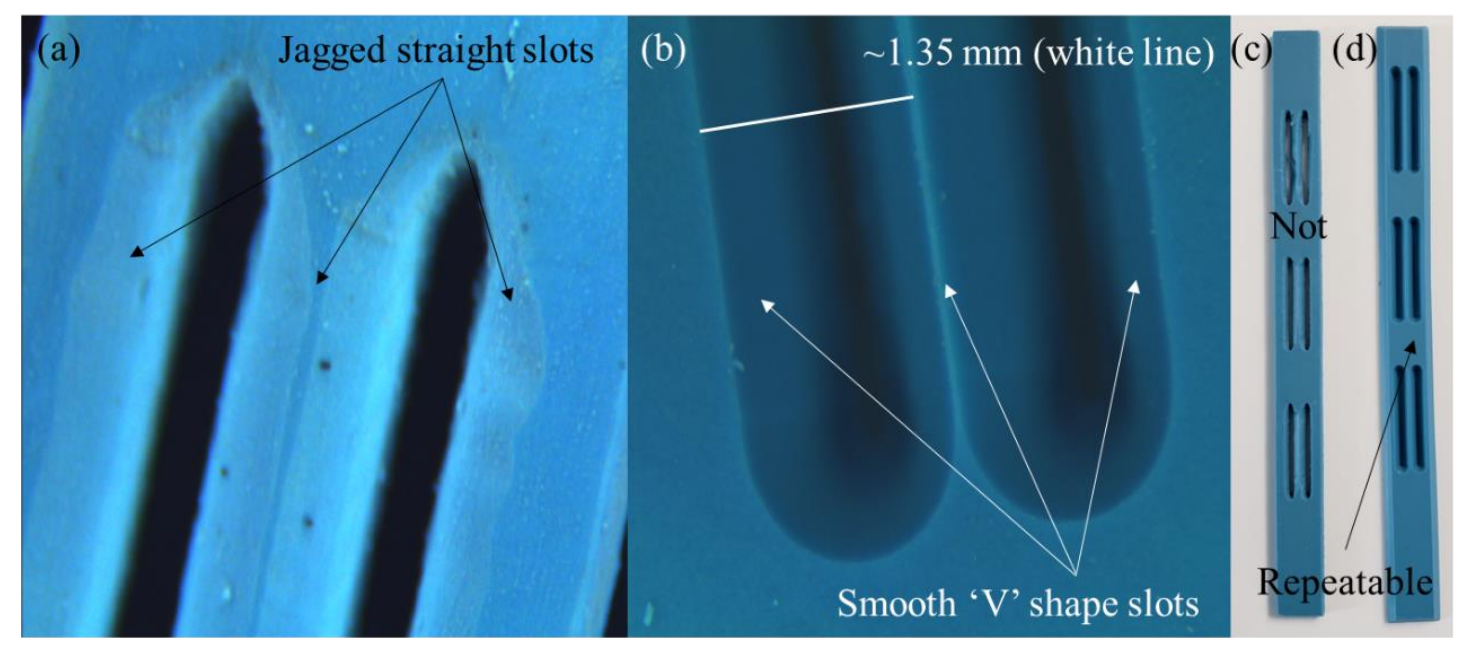

Figure 53. (a, c) Examples of laser and $(b, d)$ mill facet plates

\section{Firing Technique}

The firing technique is implemented when the cathode structure is placed in the furnace to be fired. The LTCC and silver pastes react differently to the high furnace 
temperatures which causes the cathode to warp or even crack. Most of the warping can be resolved by slow ramp rates during the firing profile, which will be shown later. The rest of the warping can be corrected by using techniques that physically prevent warping during firing.

Four different techniques were implemented and tested. The first technique was horizontal which means that the cathode was laid down horizontally in the furnace. The second technique as previously discussed [57], was the use of circular caps placed on both ends of the cathode. These caps are composed of two stacked circle layers of LTCC. The first layers have a large OD to hold the cathode upright. The second layers are the same OD as the ID of the cathode. They are sprayed with boron nitride to prevent sticking; and they shrink at the same rate as the cathode which helps maintain circularity. The third technique was a stand which the cathode would slide over and stand upright in the furnace. The stand is composed of a post and base block to keep the post upright. The post was designed with long rectangular layers of LTCC that, when laminated and fired flat, would shrink to a post having a cross sectional area of a square. Firing flat allowed for the post to be linear (as linear as the floor of the furnace), and the square crosssectional area allowed for circular support from the four corners of the square. A large rectangular base was attached to the bottom of the square post. Eventually, a four-post stand (one broke off) was fabricated which allowed for multiple cathodes to be fired at once. The fourth technique was to combine the caps and stand to create caps with stand. The cathode would slide over the stand post and then a cap would be placed on top of the cathode structure. These firing techniques are shown in Figure 54. 


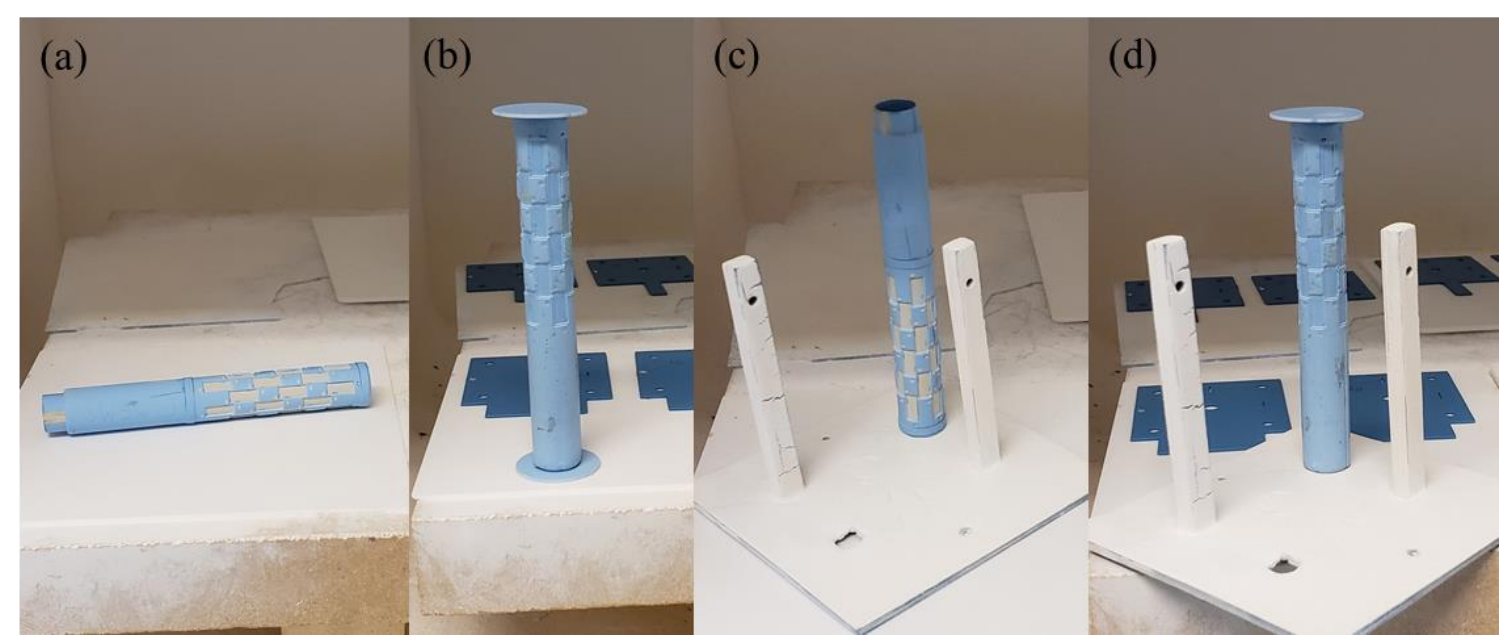

Figure 54. Firing Techniques: (a) Horizontal, (b) caps, (c) stand, and (d) caps with stand

$\underline{\text { Results }}$

Because the outcomes of each technique was not user (operator) dependent, an objective scoring system was chosen to compare the better of the two techniques for the new cathode structure firing techniques. The first scoring is circularity because the facet plates and die must fit on the cathode and be a fixed distance from the anode. The last scoring is linearity because the cathode must slide over the rod that connects to the inner (first) ground plane. The results of the scoring system are shown in Table 9.

Table 9. Objective scoring of firing techniques

\begin{tabular}{|l|c|c|c|c|}
\hline \multicolumn{1}{|c|}{ Parameters } & Horizontal & Caps & Stand & Caps with Stand \\
\hline Circularity (mm) & 0.94 & 0.63 & 0.25 & 0.78 \\
\hline Linearity (mm) & 0.30 & 1.92 & 0.24 & 1.06 \\
\hline Total (mm) & 1.24 & 2.55 & 0.49 & 1.84 \\
\hline
\end{tabular}

The scored values in Table 9 were determined by circularity and linearity measurements where zero is perfect [78]. Circularity was determined by subtracting the maximum outer diameter (OD) from the minimum OD. These measurements were taken at three points along the cathode (cathode position 1,2, and 3) and recorded 


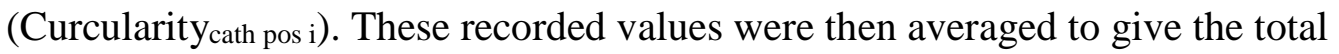
circularity of one cathode. Linearity was determined by measuring the displacement along (cathode position) the cathode structure. This measurement was accomplished with a dial indicator that was zeroed at cathode position 1 . The displacements were then measured at cathode position 2 and 3 in thousandths of an inch (mil) units. These measurements were taken at three points (Disp 1, 2, and 3) around the cathode and recorded. The stand that the cathode rested on was then measured using an assumed straight rod of aluminum stock to eliminate the errors due to the stand. The stand measurements were then added to the measurements at each cathode position to account for the stand. Both cathode position measurements (2 and 3) were then added together to give the total linearity for all three Disp positions (LinearityDisp i). All three Disp position total measurements were then averaged to give the total linearity of one cathode structure. The raw data can be found in Appendix C. Figure 55 shows a measurement diagram and the equations used to calculate the linearity and circularity for each position (i) before being averaged for each cathode.

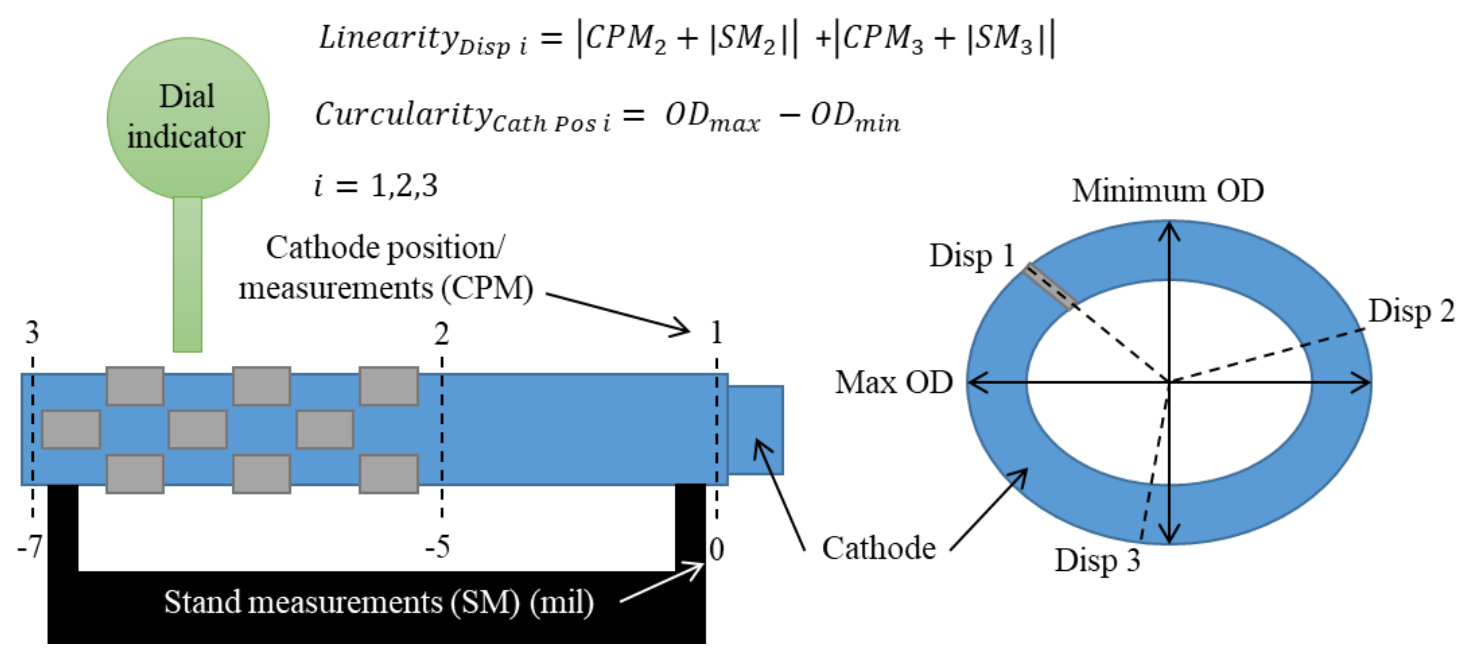

Figure 55. Firing techniques measurement diagram 
Each total measurement was calculated for two cathodes each and averaged to give circularity and linearity measurements for all four techniques. After summation of circularity and linearity for each technique, the stand technique had the smallest scored value which makes the stand firing technique the best and most optimized for the cathode manufacturing process. Figure 56a and Figure 56b show examples of bad and good circularity. Figure 56c and Figure 56d show examples of bad and good linearity.

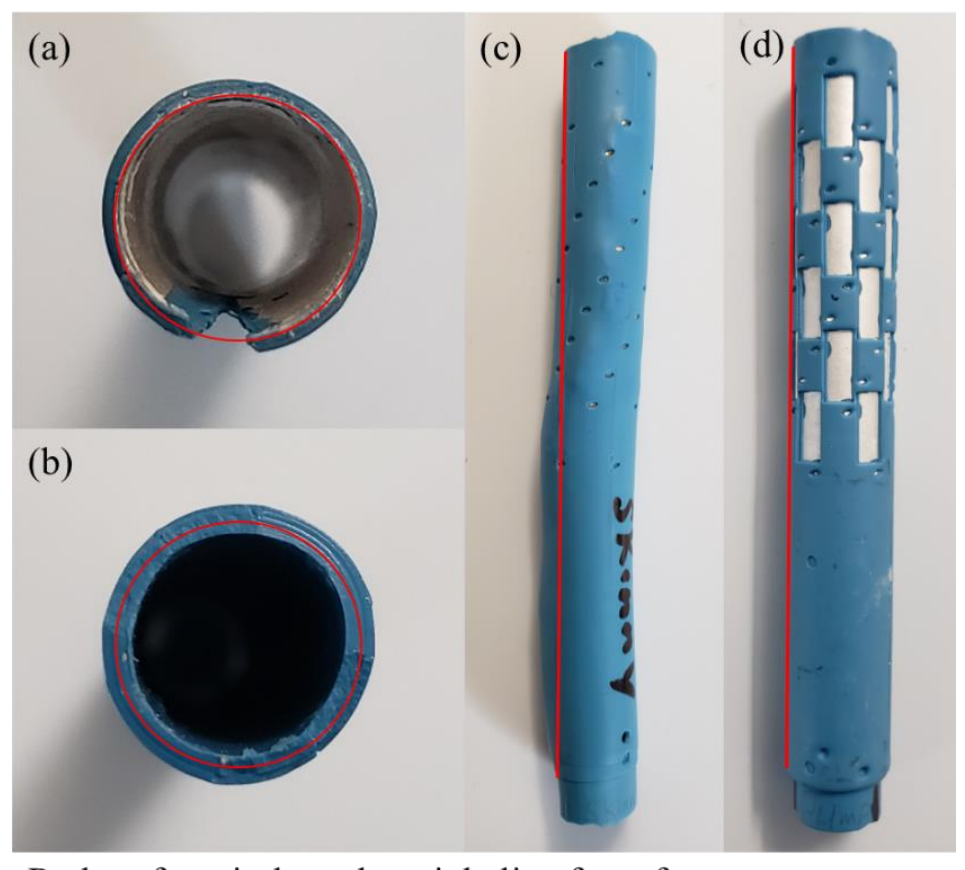

Red perfect circle and straight line for reference

Figure 56. (a, c) Examples of bad and $(b, d)$ good fired cathode structures Testing

Testing required implementing the optimal techniques for each manufacturing process in order to fabricate the new field emission cathode. When comparing LTCC wrapping techniques, the layer by group technique was optimal because it was more easily aligned, lead to tighter wraps, and was faster than the layer by layer technique. When comparing via filling techniques, the flat fill technique was optimal because it was 
less likely to short electrically and more likely to connect from the stripline to the die than the pneumatic pump technique. When comparing facet plate techniques, the mill technique was optimal because it was more repeatable and had visibly smoother ' $\mathrm{V}$ ' shaped slots than the laser technique. When comparing firing techniques, the stand technique was optimal because it had the lowest averaged circularity and linearity measurements than the other techniques.

By implementing all of the optimal techniques previously discussed, the new cathode structure was fabricated (Figure 57a) and tested. The first test was to verify with a multimeter that the vias were not shorted electrically to the ground layers which the optimized cathode passed. After verification, die were attached to the cathode (Figure 57b) and successfully lit up under a phosphor screen. This proof of concept cathode structure, proved that the cathode structure design and implementation can turn on the field emitters. Lastly, facet plates were adhered to the cathode (Figure 57c) to fully represent the final cathode design.

(a)

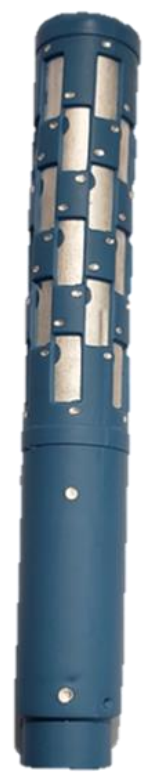

(b)

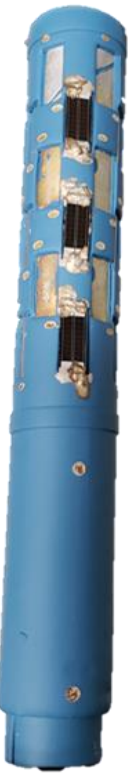

(c)

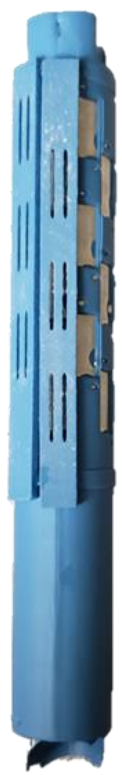

Figure 57. (a) Fired cathode structure (b) with die and (c) with facet plates 


\section{CHAPTER FOUR: STRIPLINE PROTOTYPE}

The cathode stripline (layer 2) required prototyping in order to determine the final dimensions. The purpose was to build as much of the stripline as possible so that it could be easily and rapidly implemented into the cathode structure. The finalized design will be sent out to be fabricated. The prototype stripline will be laser screen cut and screen printed in house which will be discussed later in this chapter. The first step for prototyping was to build a radio frequency test flat (RFTF) which is an elementary version of the stripline used to finalize the stripline dimensions.

\section{RF Test Flat}

The RFTF was designed to test the validity of the cathode stripline. The first study was a single stripline with one die attached. The second study was a stripline that split into five branches with one die attached to each line. The purpose of these studies was to match the model (discussed later) and to see the effects of multiple lines and multiple die on the single stripline.

In order to replicate the cathode stripline geometry, the same LTCC layers (DuPont 951) and silver paste (DuPont 6145, 6141, 6146, and Ferro 903-A) were used in the RFTF. The test flat (bottom to top) was composed of:

- Three 10-mil layers were used for structural support.

- One silver paste layer was used for a lower ground plane on the bottom of a 10-mil layer (layer 1). 
- One stripline was used for die signal drive and resistance measurements on the top of a 5-mil layer (Layer 2).

- One 10-mil and one silver paste layer was used for a top ground plane on the top of a 5-mil layer (layers 3 and 4).

- Three 10-mil layers were used for structural support as well as die slot placement (layers 5, 6, and 7).

These layers are shown in Figure 58 in order from bottom to top. The layer structure shown below is identical to the cathode layer structure except for the bottom three 10-mil structural support layers; and the RFTF is flat, whereas the cathode structure is cylindrical.

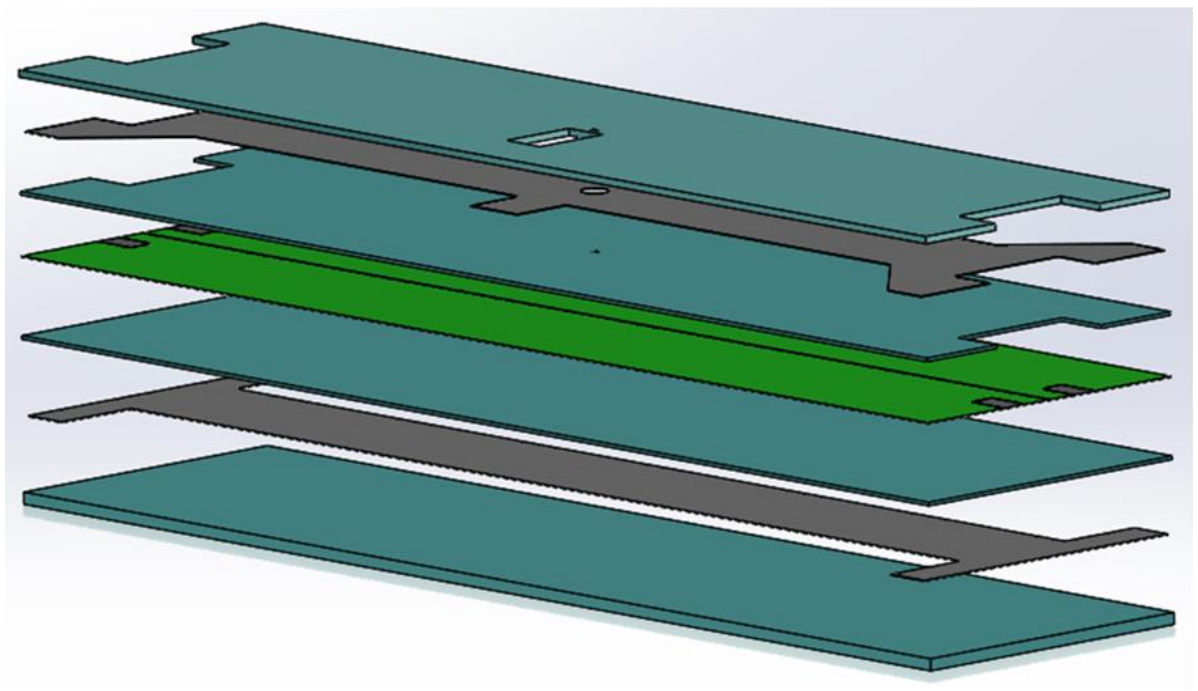

Cathode equivalent Layers 5 to 7 Ground plane 2 Layers 3 and 4 Stripline Layers 1 and 2 Ground plane 1 Structural Figure 58. RF test flat configuration

The stripline layer contains the metal stripline and soldering pads for attaching the SMA connectors. These SMA connectors allow for quick and easy impedance measurements through coaxial cables and with a network analyzer. The full fired assembly of the RFTF is shown in Figure 59. 


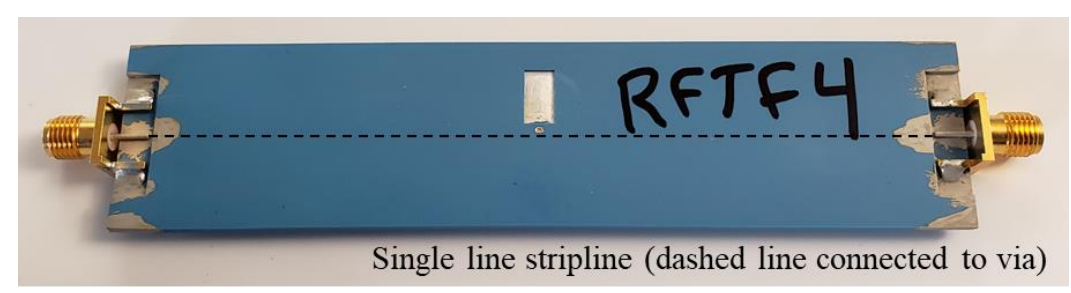

Figure 59. Fully assembled fired RFTF

The physical properties of the RFTF directly correlate to the electrical properties. By plugging in the known electrical properties and assumed thicknesses of the LTCC layers and silver paste into the model [79], shown in Figure 60, the width of the stripline was calculated in order to achieve a characteristic impedance of $\sim 50 \mathrm{Ohms}$ at a frequency of $907 \mathrm{MHz}$. The RFTF was snapped in half, and the substrate thickness was measured to be $600 \mu \mathrm{m}$ rather than $662 \mu \mathrm{m}$ as designed. The trace (metal) thickness was also measured at $5 \mu \mathrm{m}$ rather than $10 \mu \mathrm{m}$. These measured thicknesses were used in the model to determine the new fabrication stripline width to be $130 \mu \mathrm{m}$ rather than $160 \mu \mathrm{m}$. Table 10 corresponds to the model in Figure 60 which represents the ground plane 1 all the way to the ground plane 2 of the RFTF.

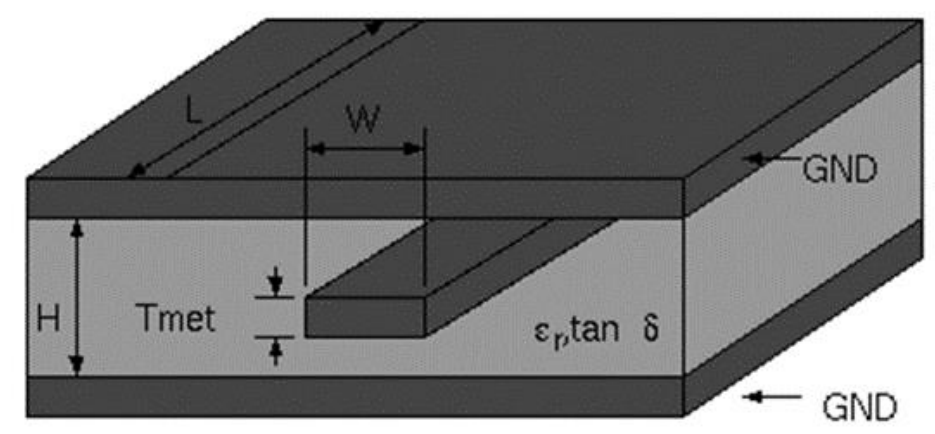

Figure 60. Cross section and components of a basic stripline diagram [79] 
Table 10. RFTF design specifications as fabricated

\begin{tabular}{|l|c|c|}
\hline \multicolumn{1}{|c|}{ Name } & Value & Units \\
\hline Metal width (W) & 130 & $\mu \mathrm{m}$ \\
\hline Trace length (L) & 11.84 & $\mathrm{~cm}$ \\
\hline Metal thickness (Tmet) & 5 & $\mu \mathrm{m}$ \\
\hline Metal resistivity (RHO) & $4.80 \mathrm{E}-07$ & Ohm-m \\
\hline Metal surface roughness (RGH) & 0.01 & $\mathrm{mil}-\mathrm{rms}$ \\
\hline Substrate thickness (H) & 600 & $\mu \mathrm{m}$ \\
\hline Relative dielectric constant (Er) & 7.8 & \\
\hline Substrate loss tangent (tand) & 0.014 & \\
\hline Frequency & 907 & $\mathrm{MHz}$ \\
\hline Characteristic Impedance & 51.401 & Ohm \\
\hline Electrical Length & 360.154 & degrees \\
\hline
\end{tabular}

After several iterations to optimize the geometry, the RFTF started to show

promising results. The closest RFTF that was built had a characteristic impedance of 49.6 $+\mathrm{j} 6.9 \Omega$ and an inductance of $1.2 \mathrm{nH}$ at the desired $907 \mathrm{MHz}$. This result was very close to the simulated $50 \Omega$ characteristic impedance. The next step was to create the RFTF with a five-line stripline, each attached to one die. The closest trial had a characteristic impedance of $48-\mathrm{j} 140 \Omega$ with an inductance of $1.1 \mathrm{pF}$. Figure 61 shows the fully assembled and fired five-line stripline RFTF.

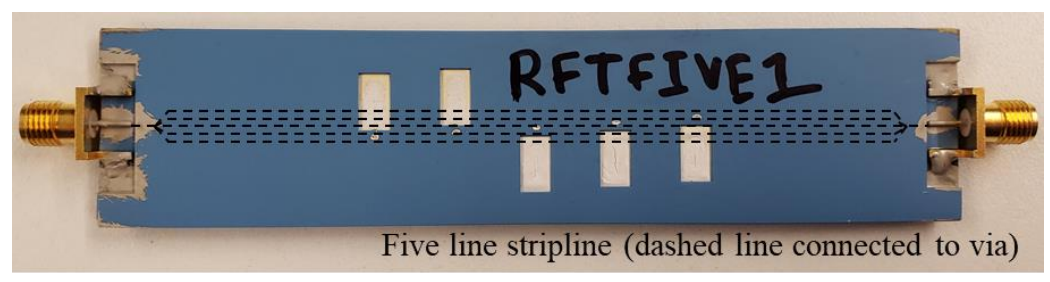

Figure 61. Five-line stripline 
The RFTF devices proved that the stripline could be manufactured from LTCC to match a RF model. The devices were also used to find ways to screen cut/print in the lab rather than outsourcing to other labs or companies. These new screen cutting/printing processes were then evaluated to test the limits of these processes. These limits would be used to determine design constraints of the cathode stripline.

\section{Laser Screen Cutting}

The initial RFTF's had lower than expected resistances which was a result of larger than expected stripline widths. These wider stripline widths were attributed to the precision of the M-300 laser that was used for cutting the stripline in the screens for screen printing. The stripline was modeled and cut as a rectangle of the exact (scaled for shrinkage) width and length of the stripline. Therefore, the actual width cut (most important and most precise) was cutting, width $+2 * \mathrm{r}$, where $\mathrm{r}$ is the radius of the laser focal point. This larger stripline width was almost 2 times the desired design width, which caused the lower resistance.

After careful calibration and measuring of the laser, the stripline was modeled as a single line with the laser focal point diameter being the width of the line. By raising and lowering the laser from the focal point, a different diameter (line width) could be obtained. Laser power and speed could also impact cut width, but these were held constant for this experiment. The smallest diameter of laser ever obtained was $\sim 90 \mu \mathrm{m}$ which was sufficient for the $130 \mu \mathrm{m}$ desired design width. By changing the height from the focal point by $\pm 0.51 \mathrm{~mm}$ or $\pm 0.25 \mathrm{~mm}$ ( \pm 0.02 in or $\pm 0.01 \mathrm{in})$, depending on the height of the laser bed, the desired $130 \mu \mathrm{m}$ cut could be obtained. 
This raising/lowering of the laser cutting was used when prototyping the stripline to achieve the desired $\sim 130 \mu \mathrm{m}$ line width on the screens. The stripline design used in the cathode structure is a barber pole pattern that spirals up the length of the structure. As previously discussed, the barber pole is designed to delay the field emission die $1 / 4$ phase which will cause all three of the die aligned vertically to emit at the same time for phase control. This angle of incline is about $6.15^{\circ}$ which is very difficult to cut using single laser diameter cuts. Originally the screen was laid down in the corner of the laser bed, aligned with the $\mathrm{X}$ and $\mathrm{Y}$ axis of the laser. This forced the laser to cut dots that lined up at the $6.15^{\circ}$ angle to achieve the angled line. The next approach was to angle the screen itself at $6.15^{\circ}$ from the $\mathrm{X}$ and $\mathrm{Y}$ axis of the laser and then perform straight cuts. This approach was much faster and resulted in continuous lines of a constant width. Figure 62a show the results of the angled screen and straight cuts. Figure $62 \mathrm{~b}$ shows the results of the straight screen and angled dot cuts.

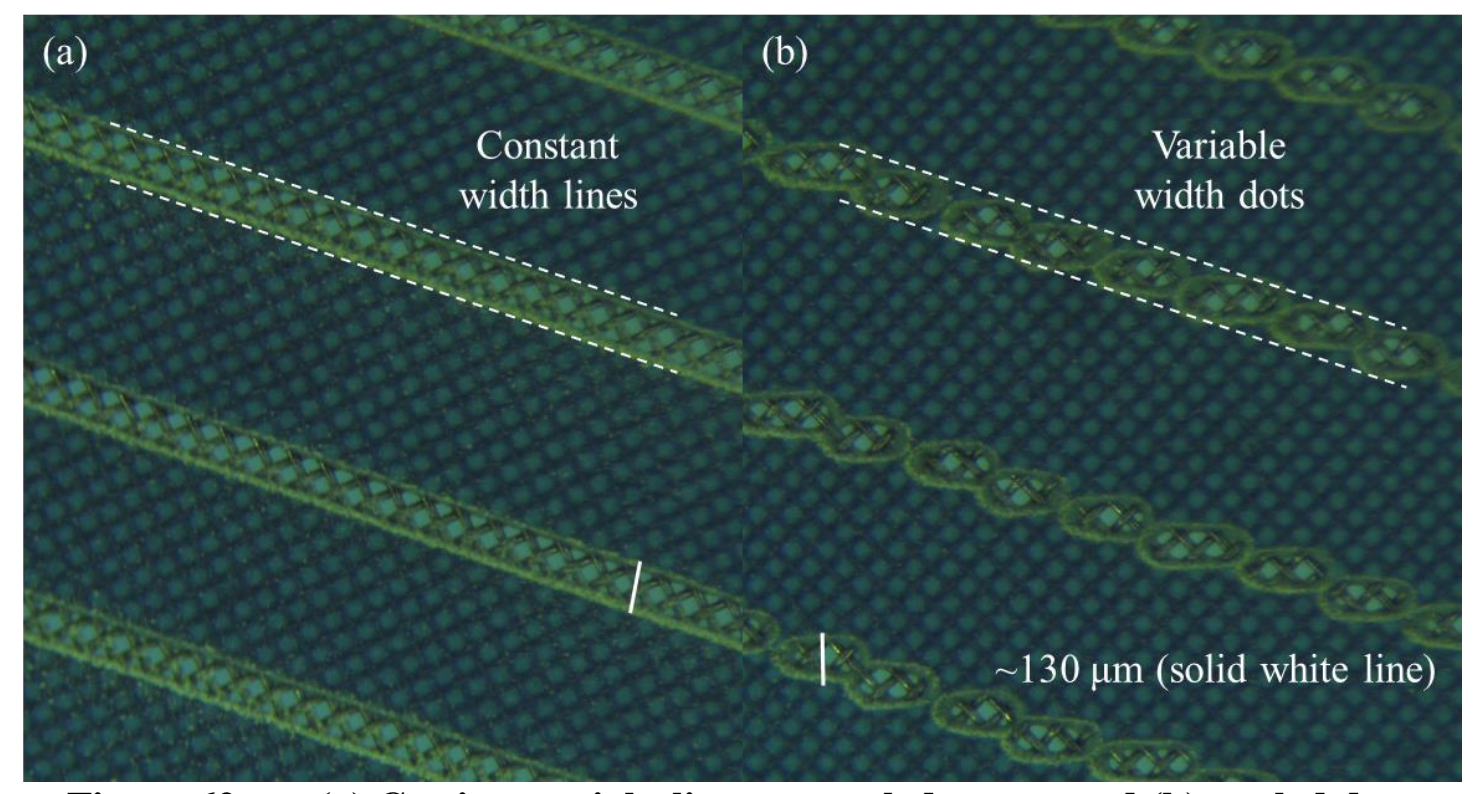

Figure 62. (a) Cutting straight lines on angled screen and (b) angled dots 


\section{Manual Screen Printing}

Some of the benefits of screen printing with a machine are edge quality and consistent layer thickness; however, these machines are expensive and time consuming to get set up properly. For rapid prototyping, one solution is to screen print by hand which is very easy and fast to set up. The downsides are the edge quality and deposition thickness. The concept is similar to printing with a machine except the screen is placed directly on top of the substrate or LTCC layer. This direct contact with the substrate causes some of the paste to stick to the screen and cause uneven layer thicknesses. The paste is gently laid on top of the open mesh and then squeegeed through by hand using one or two passes. Using more than a couple passes can force the paste to seep out beneath the mesh and increase the width of the line. Even a tiny bit of seepage can double and even triple the line width when the design width is $130 \mu \mathrm{m}$. The margin of error with one or two passes is usually about $+20 \mu \mathrm{m}$. Seepage can also occur because of the laser cutting of the screen. The laser burns the emulsion which does not give vertically straight (perpendicular to substrate) cuts. An example of hand screen printing and the seepage that can occur with more than two passes with a squeegee is shown in Figure 63 where the solid line is $\sim 130 \mu \mathrm{m}$.

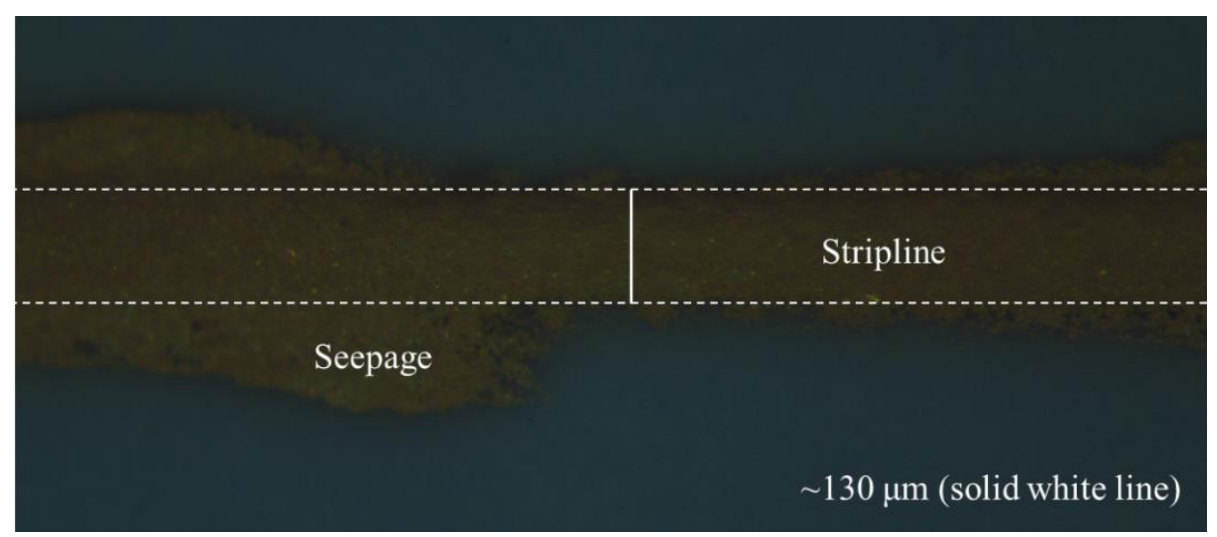

Figure 63. Example of hand screen printing 
The immediate step and most important after printing is cleaning of the screens. By laying the screen directly on the substrate, some of the paste will stick to the mesh when the screen is removed. If the screens are not cleaned properly, then the paste will dry inside the mesh and ruin the screen. The screen must be sprayed immediately with isopropyl alcohol $\left(\mathrm{C}_{3} \mathrm{H}_{8} \mathrm{O}\right.$ or IPA) and then wiped using an absorbent rag or paper towel on both sides of the screen. Spray again and wipe again with a clean cloth and repeat until the screen is visibly clear. Cleaning allows for the screen to be used multiple times with ease. Once the stripline is finalized and screen is precision UV cut, it will be used for every cathode structure which is why cleaning is so important.

The cathode structure layers are rotated $180^{\circ}$ not only for structural integrity but also to make the stripline contiguous which has to connect across the seam on layer 2. The first method was to push paste through the screens onto the edges of layer 2 . When the layer wrapped around and the two edges met, this contact would create the contiguous line, shown in Figure 64a. However, this method requires a nearly perfect wrap where the two edges meet which is rarely the case. This method was tried multiple times, and while some large sections would connect, the whole stripline (bottom to top) was never achieved.

The alternative method was to create a jumper layer on the inside of layer 3 . This jumper layer would match the lines on the outside of layer 2 and lay directly on top of them, circumventing or jumping over the seam. Figure $64 \mathrm{~b}$ and Figure $64 \mathrm{c}$ shows the jumper diagram and layers respectively, not to scale. This was tried multiple times and worked every time. Worked, in this case means, that the bottom of the cathode structure was connected to the top of the cathode structure. The only difficulty with this method is 
alignment. Given the line widths of about $130 \mu \mathrm{m}$, even the slightest misalignment vertically will ruin the whole stripline. Even if the layer 2 seams do not align correctly, the trace layer with a jumper will always connect.

(a)

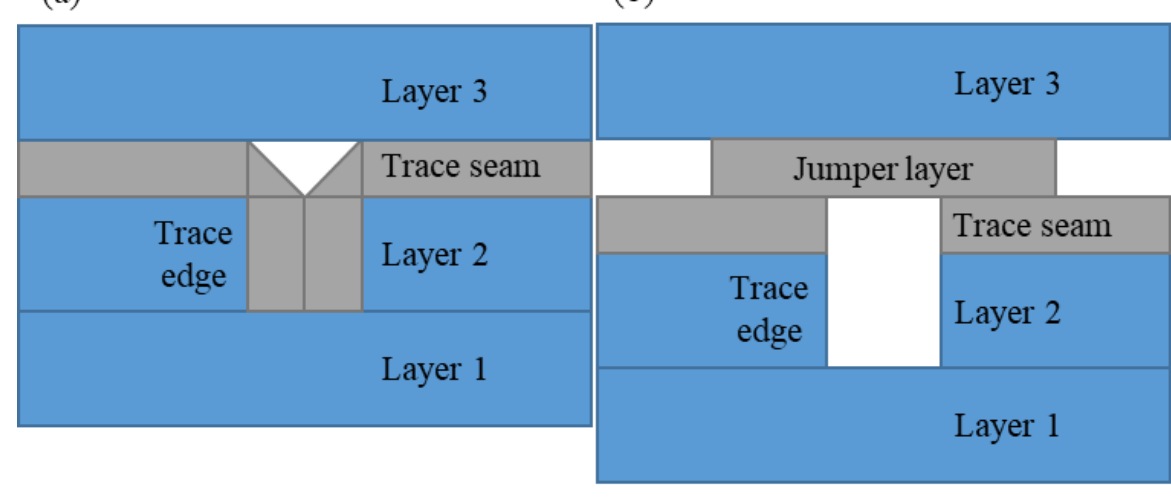

(c)

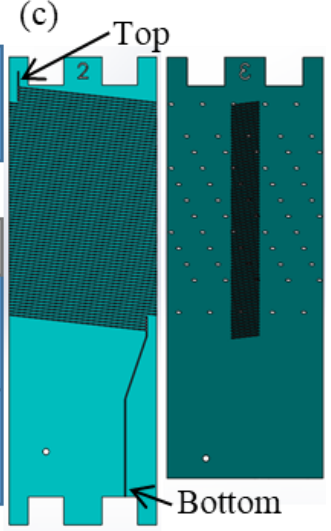

Figure 64. (a) Paste on the edge and (b) jumper layer paste diagram and (c) layers

Stripline prototyping was important to validate the theory behind the stripline design. The RFTF's were devices that proved the stripline could be manufactured using LTCC. It was also used to verify and correct some assumed values including fired thicknesses of LTCC and silver pastes. The development of laser screen cutting established minimum and controllable line widths (laser focal point diameters). It also determined new screen orientations that could be used to cut angles lines cleanly, consistently, and continuously. The development of manual screen-printing established tolerances and the importance of cleaning the screens. Lastly, the use of a jumper layer to connect across a seam was verified. 


\section{CHAPTER FIVE: CONCLUSIONS}

The LTCC material system is ideal for layered internal electrical structures such as the stripline that makes up the cathode structure. Traditionally, layers are stacked; whereas the cathode structure is composed of a novel rolled layer structure that simplified the design but not the manufacturing process. Through this research, multiple manufacturing techniques were compared and evaluated for their strengths and weaknesses. These techniques were then used to optimize the manufacturing process of the field emission cathode structure.

Prior work on LTCC rolled devices used long strips of LTCC that were rolled multiple times around a jig. These devices were not composed of vias, and the LTCC strips could withstand tension and repeated bending or rolling. This new research demonstrated that a rolled LTCC device consisting of layers (one strip of LTCC per layer), vias, and embedded circuits could be fabricated. The research resulted in two new LTCC wrapping techniques: the layer by layer and the layer by group techniques. Traditional via filling techniques required machinery and were performed on a flat surface using a specific via filling paste. This research demonstrated that via filling could be performed with various pastes, even after the layers have been rolled. Two new via filling techniques were developed: the flat fill and the pneumatic pump techniques.

Past LTCC machining techniques used a laser or mill to cut only perpendicular to the layer surface. This research demonstrated that a laser could be used to cut ' $\mathrm{V}$ ' shaped slots into the surface of LTCC. The current techniques for maintaining circularity while 
being fired consisted of removing the top and bottom of the device as well as the use of circular caps. This research demonstrated that circularity and linearity of a rolled device could be maintained while being fired. Four different firing techniques were studied: the horizontal, the caps, the stand, and the stand with caps techniques.

The layer by layer wrapping technique proved very useful when rolling sensitive or weak layers but was time consuming and tedious. The layer by group wrapping technique proved very easy to align and useful when rolling strong layers very rapidly and tightly, but it was ineffective for weak layers. The flat fill via filling technique proved very useful for completely and precisely filled vias, but it was slower depending on the paste used. The pneumatic pump via filling technique proved very useful for rapid via filling, but it tended to spill over the edges and not completely fill the vias.

The laser facet plate manufacturing technique proved very easy to master but was extremely inconsistent and entirely user dependent. The mill facet plate manufacturing technique proved to be extremely accurate and repeatable, but very slow. The horizontal technique when firing the cathode proved to increase linearity but not circularity due to the flat spot on one side. The caps technique when firing increased circularity but would split the cathode structure often due to the thin layers (layer 1, 5-mil and layer 2, 10-mil) at the bottom of the cathode. The square stand technique when firing proved to increase circularity and linearity due to the linear (fired flat) and concentric (square) post. The square stand with caps technique when firing proved to increase linearity but only occasionally increase circularity due to splitting of the cathode.

Prototyping the stripline led to elementary versions of the stripline (RFTF) being developed and tested. The single line RFTF was developed which matched the model 
characteristic impedance. The five-line RFTF was developed and tested but never modeled to verify accuracy. Prototyping also led to fairly precise screen cutting and printing techniques for in house prototyping. It also led to proving the concept of the jumper layer to keep the stripline contiguous across the seams. These developments should allow for easy integration of the stripline once the design has been finalized.

The best techniques for each process were compared and implemented to give the optimized cathode structure manufacturing process. This process was used to build proof of concept cathode structures that would not short electrically and would drive the field emission die when under a phosphor screen. Again, these cathodes were manufactured to easily integrate the stripline once designed. The step by step cathode manufacturing process is discussed in great detail in Appendix A.

Future work would include finalizing the stripline design. The purpose of the RFTF was to learn what happens when a single stripline splits into five traces and when multiple die are attached to a single stripline. The $50 \Omega$ resistor that the stripline was to terminate also not produced due to the lack of resistive paste. Using the paste to design the accurate $50 \Omega$ using screen printing would also allow for easy transition to the full stripline into the cathode. 


\section{REFERENCES}

[1] Y. Blanchard, G. Galati and P. V. Genderen, "The Cavity Magnetron: Not Just a British Invention [Historical Corner]," IEEE Antennas and Propagation Magazine, vol. 55, no. 5, pp. 244-254, 2013.

[2] H. Huang, K. Huang and C. Liu, "Experimental Study on the Phase Deviation of 20-kWS-Band CW Phase-Locked Magnetrons," IEEE Microwave and Wireless Components Letters, vol. 28, no. 6, pp. 506-511, 2018.

[3] R. Adler, "A study of locking phenomena in oscillators," Proceedings of the IEEE, vol. 61, no. 10, pp. 1380-1385, 1973.

[4] H. Sze, D. Price and B. Harteneck, "Phase locking of two strongly coupled vircators," Journal of Applied Physics, vol. 67, no. 5, pp. 2278-2282, 1998.

[5] J. Benford, H. Sze, W. Woo, R. R. Smith and B. Harteneck, "Phase Locking of Relativistic Magnetrons," Physical Review Letters, vol. 62, no. 8, pp. 969971, 1989.

[6] J. J. Choi and G. W. Choi, "Experimental Observation of Frequency Locking and Noise Reduction in a Self-Injection-Locked Magnetron," IEEE Transactions on Electron Devices, vol. 54, no. 12, pp. 3430-3432, 2007.

[7] E. J. Cruz, B. W. Hoff, P. Pengvanich, Y. Y. Lau, R. M. Gilgenbach and J. W. Luginsland, "Experiments on peer-to-peer locking of magnetrons," Applied Physics Letters, vol. 95, no. 19, pp. 191503-1-3, 2009. 
[8] P. Pengvanich, Y. Y. Lau, E. Cruz, R. M. Gilgenbach, B. Hoff and J. W. Luginsland, "Analysis of peer-to-peer locking of magnetrons," Physics of Plasmas, vol. 15, no. 10, pp. 103104-1-4, 2008.

[9] J. S. Levine, N. Aiello, J. Benford and B. Harteneck, "Design and operation of a module of phase-locked relativistic magnetrons," Journal of Applied Physics, vol. 70, no. 5, pp. 2838-2848, 1998.

[10] J. Benford, "History and future of the relativistic magnetron," in 2010 International Conference on the Origins and Evolution of the Cavity Magnetron, Bournemouth, IEEE, 2010, pp. 40-45.

[11] J. Browning and J. Watrous, "Faceted magnetron concept using field emission cathodes," Journal of Vacuum Science \& Technology B, vol. 29, no. 2, pp. 02B109-1-7, 2011.

[12] S. Fernandez-Gutierrez, J. Browning, M.-C. Lin, D. N. Smithe and J. Watrous, "Simulation of a rising sun magnetron employing a faceted cathode with a continuous current source," Journal of Vacuum Science \& Technology B, vol. 32, no. 6, pp. 061205-1-6, 2014.

[13] J. Browning, S. Fernandez-Gutierrez, M.-C. Lin, D. N. Smithe and J. Watrous, "Phase control and fast start-up of a magnetron using modulation of an addressable faceted cathode," Applied Physics Letters, vol. 104, no. 23, pp. 233507-1-5, 2014.

[14] S. Fernandez-Gutierrez, J. Browning, M.-C. Lin, D. N. Smithe and J. Watrous, "Phase-control of a rising sun magnetron using a modulated, addressable, current," Journal of Vacuum Science \& Technology B, vol. 33, no. 3, pp. 031203-1-7, 2015.

[15] S. A. Fernandez-Gutierrez, "Simulation of a Magnetron using Discrete Modulated Current Sources," Boise State University, Boise, 2014. 
[16] S. Fernandez-Gutierrez, J. Browning, M.-C. Lin, D. N. Smithe and J. Watrous, "Dynamic phase-control of a rising sun magnetron using modulated and continuous," Journal of Applied Physics, vol. 119, no. 4, pp. 044501-1-5, 2016.

[17] A. W. Hull, "The Effect of A Uniform Magnetic Field on the Motion of Electrons Between Coaxial Cylinders," American Physical Society Physical Review, vol. 18 , no. 1 , pp. $31-57,1921$.

[18] H. Yagi, "Beam Transmission of Ultra Short Waves," Proceedings of the Institute of Radio Engineers, vol. 16, no. 6, pp. 715-740, 1928.

[19] M. H. F. Wilkins, "John Turton Randall, 23 March 1905 - 16 June 1984," Biographical Memoirs of Fellows of the Royal Society, vol. 33, pp. 493$535,1987$.

[20] B. S. Lovell, Echoes of War: The Story of H2S Radar, Boca Raton: CRC Press, 1991.

[21] B. S. Lovell, "The cavity magnetron in World War II: was the secrecy justified?," Notes and Records, vol. 58, no. 3, pp. 283-294, 2004.

[22] E. G. Bowen, Radar Days, Boca Raton: CRC Press, 1998.

[23] S. Phelps, The Tizard Mission: The Top-Secret Operation That Changed the Course of World War II, Yardley: Westholme Publishing, 2010.

[24] H. A. H. Boot and J. T. Randall, "The Cavity Magnetron," Journal of Applied Physics*, pp. 928-938, 1946.

[25] J. T. Dolan, M. G. Ury and C. H. Wood, "Lamp including sulfur". United States of America Patent US5404076A, 25 October 1990.

[26] D. A. MacLennan, B. P. Turner, J. T. Dolan, M. G. Ury and P. Gustafson, "Efficient, Full-Specrum, Long- Lived, non-Toxic Microwave Lamp for 
Plant Growth," in International Lighting in Controlled Environments

Workshop, Cape Canaveral, NASA-CP-95-3309, 1994, pp. 243-254.

[27] F. A. Bassyouni, S. M. Abu-Bakr and M. A. Rehim, "Evolution of microwave irradiation and its application in green chemistry and biosciences," Research on Chemical Intermediates, vol. 38, no. 2, pp. 283-322, 2012.

[28] P. L. Spencer, "Method of treating foodstuffs". United States of America Patent US2495429A, 8 October 1945.

[29] J. M. Osepchuk, "The magnetron and the microwave oven: A unique and lasting relationship," in 2010 International Conference on the Origins and Evolution of the Cavity Magnetron, Bournemouth, IEEE, 2010, pp. 46-51.

[30] E. Gorstein, "How do microwaves really work?," Kiwi Report, 5 July 2017. [Online]. Available: http://www.kiwireport.com/how-do-microwaveswork/. [Accessed 2 September 2018].

[31] M. D. Forno, "Magnetron," deei, 29 May 2017. [Online]. Available: https://sites.google.com/a/deei.units.it/massimodalforno/home/electromagnetic-theory-devices/magnetron. [Accessed 2 September 2018].

[32] "Magnetron - Construction and Operation," Wiki for You, 23 May 2013. [Online]. Available: http://www.wikiforu.com/2013/05/magnetron-construction-andoperation.html. [Accessed 2 September 2018].

[33] C. R. Nave, "The Magnetron," Georgia State University HyperPhysics, [Online]. Available: http://hyperphysics.phyastr.gsu.edu/hbase/Waves/magnetron.html. [Accessed 2 September 2018].

[34] C. Wolff, "Magnetron," Radartutorial, [Online]. Available: http://www.radartutorial.eu/08.transmitters/Magnetron.en.html. [Accessed 2 September 2018]. 
[35] H. Sze, R. R. Smith, J. Benford and B. D. Harteneck, "Phase-Locking of Strongly Coupled Relativistic Magnetrons," IEEE Transactions on Electromagnetic Compatibility, vol. 34, no. 3, pp. 235-241, 1992.

[36] W. Ockenga, "Phase Contrast," Leica Microsystems, 9 June 2011. [Online]. Available: https://www.leica-microsystems.com/science-lab/phasecontrast/. [Accessed 2 September 2018].

[37] C. Moler, MATLAB, Natick: MathWorks, 2018.

[38] Peppergrower, "Phase shift," Wikimedia Commons, 21 February 2009. [Online]. Available: https://commons.wikimedia.org/wiki/File:Phase_shift.svg. [Accessed 2 September 2018].

[39] MIT, Vacuum Gatted Field Emitters, Cambridge: Massachusetts Institute of Technology, 2018.

[40] E. Media, "Symmetric Stripline Impedance Calculator," All About Circuits, [Online]. Available: https://www.allaboutcircuits.com/tools/symmetricstripline-impedance-calculator/. [Accessed 2 September 2018].

[41] L3-Technologies, Magnetron Drawings and Diagrams, Torrance: L3 Electron Technologies Inc, 2018.

[42] "Magnetron Resonators," Klystrons and Magnetrons, [Online]. Available: http://www.cdvandt.org/Strapping\%20of\%20Magnetrons.pdf. [Accessed 2 September 2018].

[43] DuPont, Green Tape 951 Low Temperature Ceramic System, Wilmington, 2011.

[44] D. Black, L. Schoensee, J. Richardson, T. Vleisides, N. Kempf, D. Wang, Z. Ren and Y. Zhang, "Power Generation from Nanostructured Half-Heusler Thermoelectrics for Efficient and Robust Energy Harvesting," ACS Applied Energy Materials, vol. 1, no. 11, p. 5986-5992, 2018. 
[45] J. Lau, C. P. Wong, J. L. Prince and W. Nakayama, Electronic Packaging: Design, Materials, Process, and Reliability, New York: McGraw-Hill, 1998.

[46] Y. Imanaka, Multilayered Low Temperature Cofired Ceramics (LTCC) Technology, New York: Springer Science+Business Media, 2005.

[47] D. G. Plumlee, "Fabrication of a Miniature Ion Mobility Spectrometer in Low Temperature Co-fired Ceramics," Boise State University, Boise, 2003.

[48] A. Beikmohamadi, P. Graddy, D. Nair, J. Parisi and S. Stewart, "Plating reliability and high frequency testing of DuPont ${ }^{\mathrm{TM}}$ GreenTape ${ }^{\mathrm{TM}}$ 9K7 LTCC," International Symposium on Microelectronics, vol. 2013, no. 1, pp. 678682, 2013.

[49] Ferro, 903-A Cermet Silver Conductor, King of Prussia: ElectroScience Laboratories, 2016.

[50] DuPont, 6145 Silver Cofireable Conductor, Wilmington, 2012.

[51] DuPont, 6141 Silver Cofireable Via Fill, Wilmington, 2012.

[52] DuPont, 6146 Silver/Palladium Cofireable Solderable Conductor, Wilmington, 2012.

[53] DuPont, CF011 CF Series Buried Resistors, Wilmington, 2013.

[54] J. Browning, M. Pearlman, D. Plumlee, T. Akinwande, C. Armstrong and M. Worthington, "Phase-Controlled Magnetron Development," L3 EDD Communications, Boise, 2018.

[55] M. Pearlman, T. Rowe and J. Browning, "Simulation of Electron Hop Funnel Hysteresis," IEEE Transactions on Plasma Science, vol. 41, no. 8, pp. 2291-2298, 2013.

[56] K. A. Peterson, K. D. Patel, C. K. Ho, S. B. Rohde, C. D. Nordquist, C. A. Walker, B. D. Wroblewski and M. Okandan, "Novel Microsystem Applications with 
New Techniques in Low-Temperature Co-Fired Ceramics," International Journal of Applied Ceramic Technology, vol. 2, no. 5, pp. 345-363, 2005.

[57] K. Parrish, M. Yates, D. Reis, D. Plumlee and J. Taff, "Characterization of the Fabrication Process of Rolled LTCC Structures," International Journal of Applied Ceramic Technology, vol. 10, no. 3, pp. 458-467, 2013.

[58] K. Peterson, S. B. Rohde, K. B. Pfeifer and T. S. Turner, "Novel LTCC fabrication techniques applied to a rolled micro ion mobility spectrometer," Electrochemical Society Proceedings, vol. 2003, no. 27, pp. 156-171, 2003.

[59] J. Browning, C. Lee, D. Plumlee, S. Shawver, S. M. Loo, M. Yates, M. McCrink and J. Taff, "A Miniature Inductively Coupled Plasma Source for Ion Thrusters," IEEE Transactions on Plasma Science, vol. 39, no. 11, pp. 3187-3195, 2011.

[60] D. Krueger, K. Peterson and L. Euler, "Electromagnetic Isolation Solutions in Low Temperature Cofired Ceramic (LTCC)," International Symposium on Microelectronics, vol. 2011, no. 1, pp. 760-767, 2011.

[61] M. Kakimoto, "Punch press for piercing green sheet with liner". United States of America Patent US4990080A, 29 June 1988.

[62] G. Baccini, "Device to produce multi-layer electronic circuits". United States of America Patent US6370748B1, 2 April 1999.

[63] G. Wang, E. C. Folk, F. Barlow and A. Elshabini, "Fabrication of microvias for multilayer LTCC substrates," IEEE Transactions on Electronics Packaging Manufacturing, vol. 29, no. 1, pp. 32-41, 2006.

[64] C. Q. Scrantom and J. C. Lawson, "LTCC technology: where we are and where we're going. II," in 1999 IEEE MTT-S International Topical Symposium on Technologies for Wireless Applications, Vancouver, IEEE, 2002, pp. 193200. 
[65] M. A. Girardi, K. A. Peterson and P. T. Vianco, "LTCC Thick Film Process Characterization," Additional Conferences, vol. 2016, no. CICMT, pp. 142$150,2016$.

[66] F. E. Materials, "LTCC Processing Instructions for Ferro A6 and L8 Tape," FERRO Electronic Materials, [Online]. Available: http://www.ferro.com/noncms/ems/EPM/content/docs/Ferro\%20LTCC\%20Design\%20Guide.pdf. [Accessed 2 September 2018].

[67] "DuPont ${ }^{\mathrm{TM}}$ GreenTape ${ }^{\mathrm{TM}}$ Design and Layout Guidelines," DuPont, 2009. [Online]. Available: http://www.dupont.com/content/dam/dupont/products-andservices/electronic-and-electricalmaterials/documents/prodlib/GreenTape_Design_Layout_Guidelines.pdf. [Accessed 2 September 2018].

[68] D. Jurkow and L. Golonka, "Application of Design of the Experiment in Preliminary Investigations on the end Milling of Low Temperature Co-fired Ceramics," International Journal of Applied Ceramic Technology, vol. 10, no. 4, pp. 671-681, 2013.

[69] D. Plumlee, J. Steciak and A. Moll, "Development of a micro-nozzle and ion mobility spectrometer in LTCC," in 2004 IEEE Workshop on Microelectronics and Electron Devices, Boise, IEEE, 2004, pp. 95-98.

[70] K. M. Nowak, H. J. Baker and D. R. Hall, "Cold processing of green state LTCC with a CO2 laser," Applied Physics A, vol. 84, no. 3, pp. 267-270, 2006.

[71] E. Xin, "China with Different Coating Solid Carbide End Mills," Changzhou Xute Jifeng Tools Group Co., Ltd., 2013. [Online]. Available: https://xutejifeng.en.made-in-china.com/product/SXIQihtYJWkx/ChinaChina-with-Different-Coating-Solid-Carbide-End-Mills.html. [Accessed 2 September 2018]. 
[72] M. Hayajneh, M. S. Tahat and B. Joachim, "A Study of the Effects of Machining Parameters on the Surface Roughness in the End-Milling Process," Jordan Journal of Mechanical and Industrial Engineering, vol. 1, no. 1, pp. 1-5, 2007.

[73] "How Does Laser Cutting Work?," ESAB, 2018. [Online]. Available:

http://www.esabna.com/us/en/education/blog/how-does-laser-cuttingwork.cfm. [Accessed 2 September 2018].

[74] "Emulsion," Riv Inc, [Online]. Available: http://rivinc.com/wp2/emulsion/. [Accessed 2 September 2018].

[75] "Thick Film Technology," IDC Technologies, [Online]. Available: http://www.idconline.com/technical_references/pdfs/electronic_engineering/Thick_film_te chnology.pdf. [Accessed 2 September 2018].

[76] P. K. Khanna, B. Hornbostel, M. Burgard, W. Schafer and J. Dorner, "Studies on three-dimensional moulding, bonding and assembling of low-temperaturecofired ceramics for MEMS and MST applications," Materials Chemistry and Physics, vol. 89, no. 1, pp. 72-79, 2005.

[77] M. F. Shafique, A. Laister, M. Clark, R. E. Miles and I. D. Robertson, "Fabrication of embedded microfluidic channels in low temperature co-fired ceramic technology using laser machining and progressive lamination," Journal of the European Ceramic Society, vol. 31, no. 13, pp. 2199-2204, 2011.

[78] A. Neumann, Geometric Dimensioning and Tolerancing Workbook, Longboat Key: Technical Consultants Incorporated, 1995.

[79] D. McMahill, "Symmetric Stripline Analysis/Synthesis Calculator," CGI-Wcalc, 11 January 2009. [Online]. Available: http://wcalc.sourceforge.net/cgibin/stripline.cgi. [Accessed 2 September 2018]. 
APPENDIX A: CATHODE MANUFACTURING 
This appendix will cover the completely optimized manufacturing process of the vacuum gated field emission cathode composed of LTCC. It will discuss in depth with pictures: cutting of the seven LTCC layers, cleaning, applying the silver paste to the surfaces through screen printing as well as via filling, wrapping the layers around the jig, the lamination process, the firing structure and profile, and finally the facet plate manufacturing process.

\section{Layer Cutting}

The first step is to peel off the white mylar paper from one 10 mil and one 5 mil 8'x8' sheet of LTCC and place them top sides up, side by side into the laser cutter bed shown in Figure 65. The top side is the matte blue and the bottom is the shinier blue that was attached to the mylar paper.

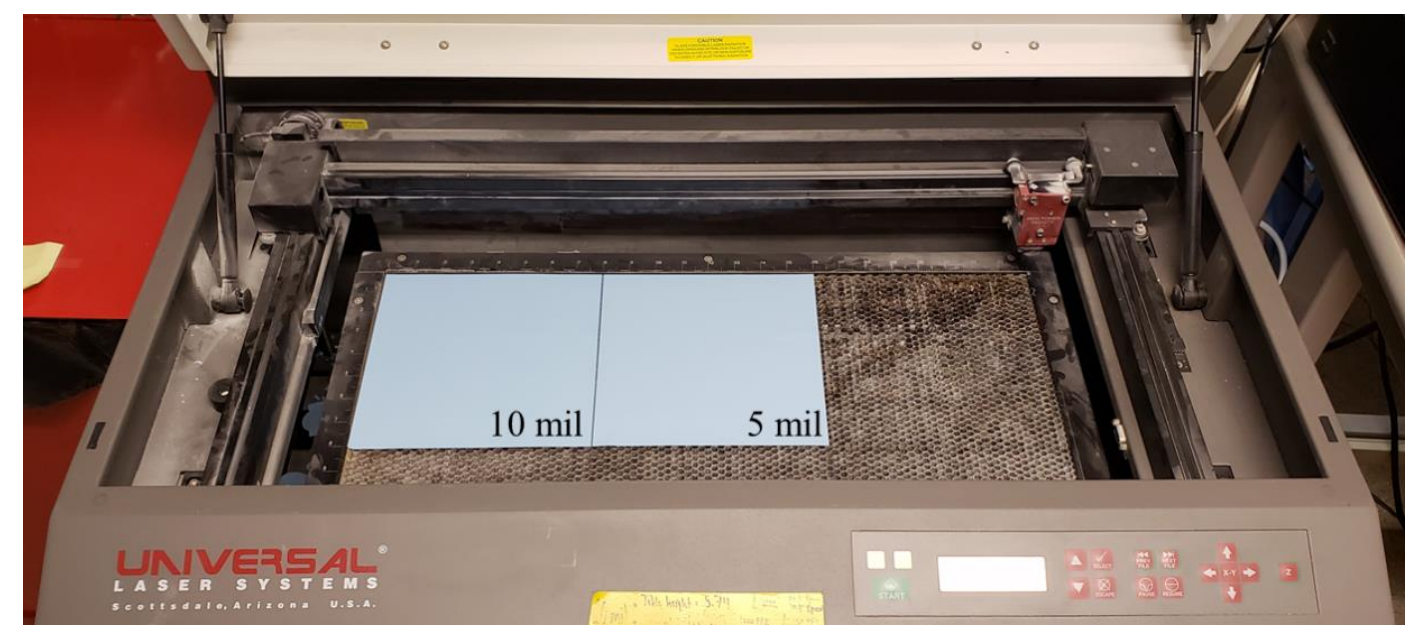

Figure 65. Position of LTCC on laser bed

Next, open CorelDraw and load the current layer configuration, 'Mark V_4-718.cdr'. This configuration has two sets of layers to produce two cathodes, for explanation purposes, only one set of layers was cut out, shown in Figure 66. 


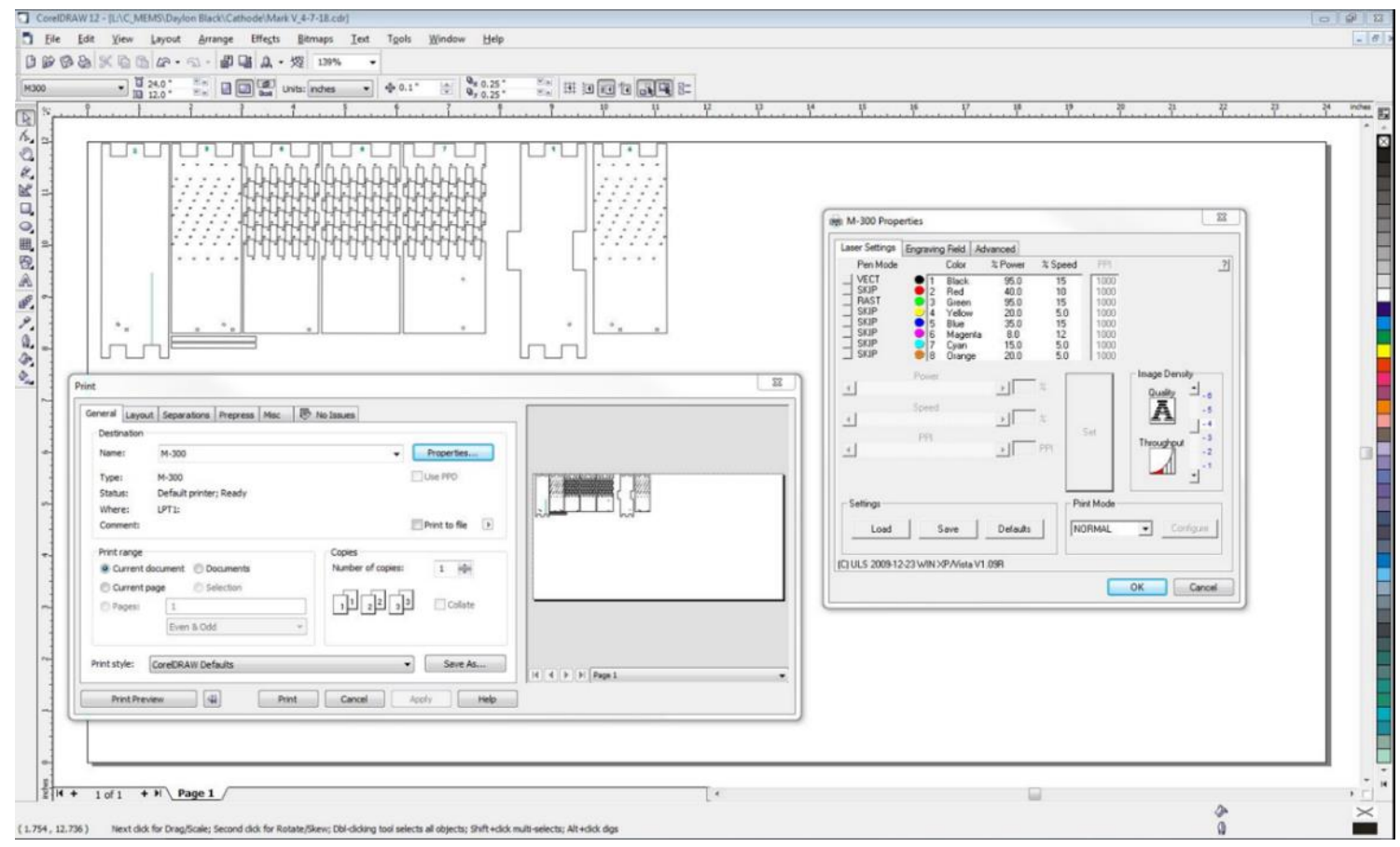

Figure 66. Printer settings

Before printing, make sure all of the CorelDraw line widths are 'hairline' and that all of the line colors are 'black' and 'green' where appropriate. After clicking print, make sure the printer is 'M-300', then click 'Properties...'. In the properties window, click and change all of the color settings to 'skip' EXCEPT black and green which need to be changed to the settings shown in Table 11. Next, click 'OK', 'Apply', then 'Print' to print.

Table 11. Black and green printer settings

\begin{tabular}{|c|c|c|c|c|}
\hline Pen Mode & Color & $\%$ Power & $\%$ Speed & PPI \\
\hline VECT & Black & 95.0 & 15 & 1000 \\
\hline RAST & Green & 95.0 & 15 & 1000 \\
\hline
\end{tabular}

Go over to the laser cutter and press the ' $Z$ ' button, and then the up and down arrows next to 'SELECT' to adjust the Z-height to the correct single sheet of LTCC cutting height. Pressing 'SELECT' will change the height increments from tenths to 
hundredths. Press ' $Z$ ' to accept the new changes. The Z-height number should be written next to the laser and on the white board; at the time the height was -3.08 as shown in Figure 67a. Press the 'NEXT FILE' repeatedly until the desired file name appears as shown in Figure 67b. Press the 'START' button to begin cutting.

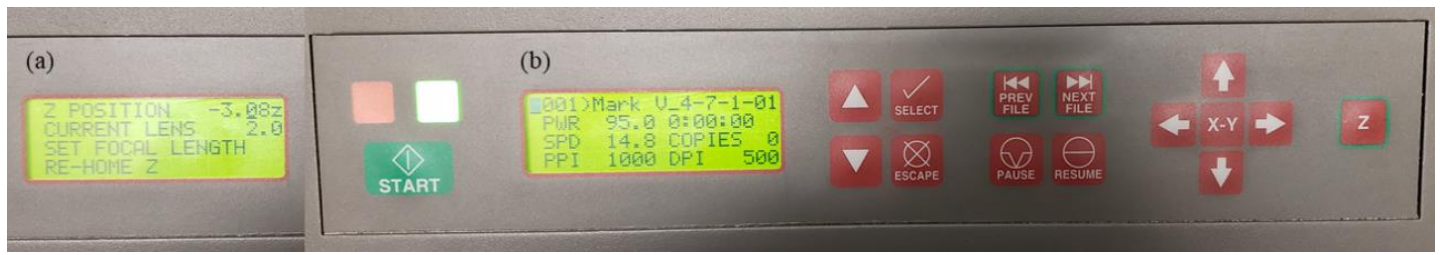

Figure 67. (a) Laser cutter menu Z-height and (b) file

After the laser has finished cutting, the sheets will look like those shown in Figure 68. Remove the layers from the bed and place them on a tray for cleaning. Remove the large scraps of LTCC and place them in the 10 and 5 mil scrap bags respectively. Throw the smaller scraps away in the red LTCC/Silver disposable bin.

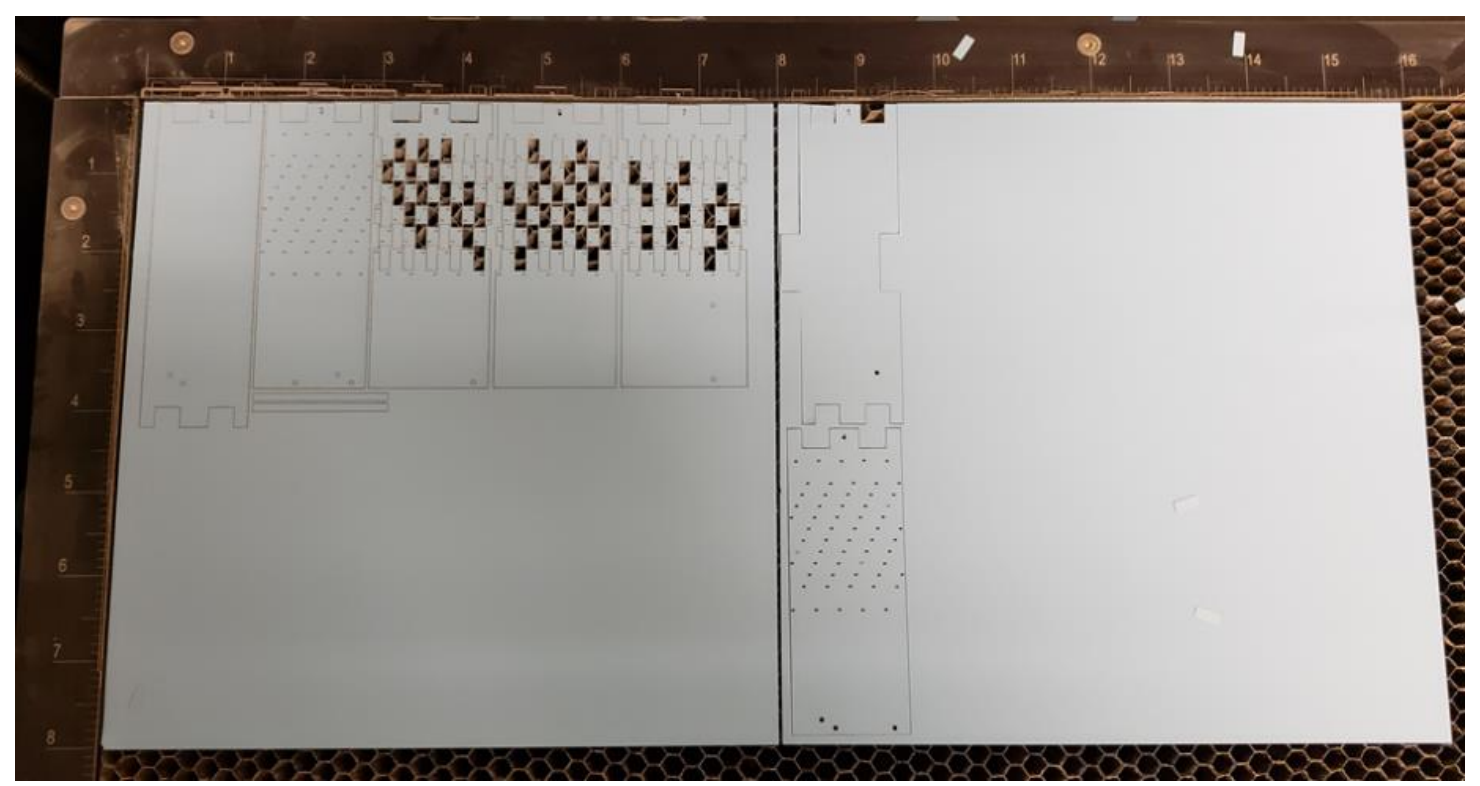

\section{Figure 68. Cut sheets of LTCC for one cathode}

NOTES: Slowing the laser cutter speed down will cut out the vias better however, it will weaken and break the thin strips holding the rectangles together. The only green on 
the layers are the numbers which may be changed to the color black and cut on 'VECT'. 'VECT' cuts all the way through the layer and 'RAST' produces surface cuts which is primarily used for engraving. Save the larger scraps of LTCC and use often. Do not waste the LTCC.

\section{Cleaning}

The paste will not fully adhere to the insides of the vias if they are not cleaned correctly which is why cleaning is very important. With all the layers flat on the tray, use the sponge, Figure 69b, to gently wipe most if not all of the burned LTCC debris (soot) from the layers shown in Figure 69a. This will loosen the remaining rectangles which should fall out once picked up.

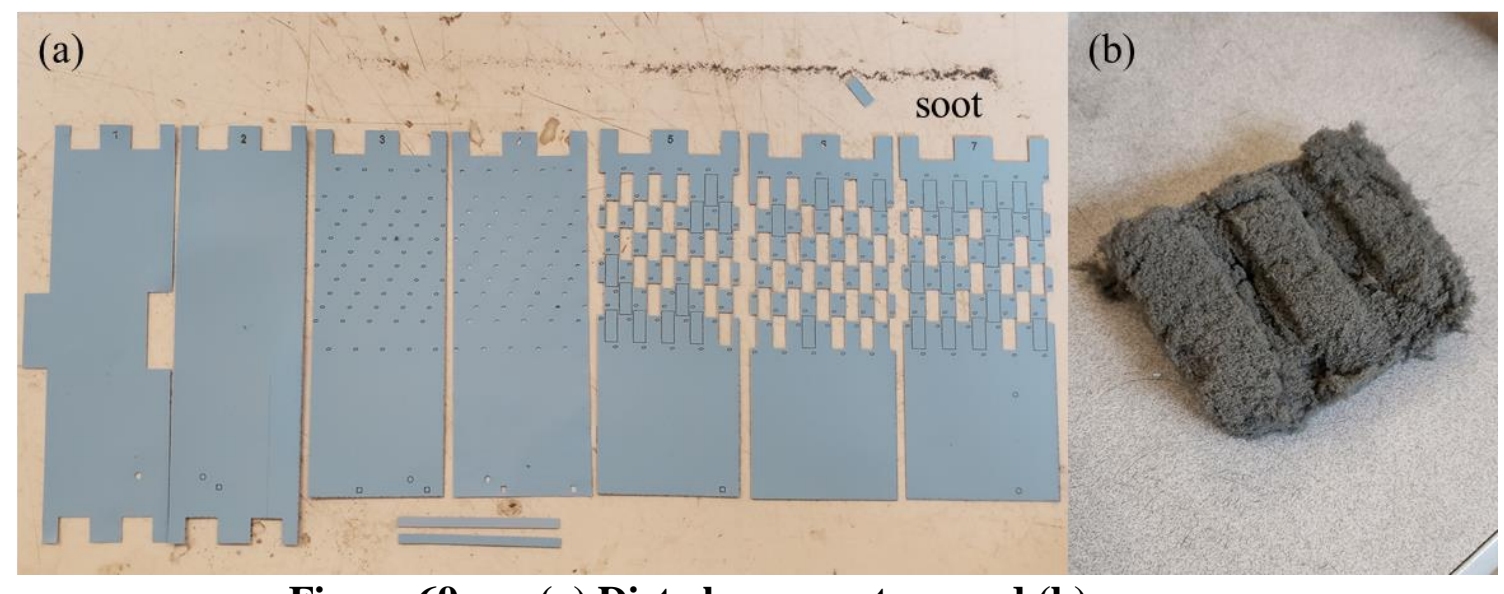

Figure 69. (a) Dirty layers on tray and (b) sponge

Using a pick or sharp tweezers, gently pick up the layers and push the vias out of the holes without breaking the layers shown in Figure 70. Then wipe the layers with the sponge again to remove any excess soot. Lay the layers with vias back on the tray and gently rub with a finger to remove the soot still stuck in the vias. 


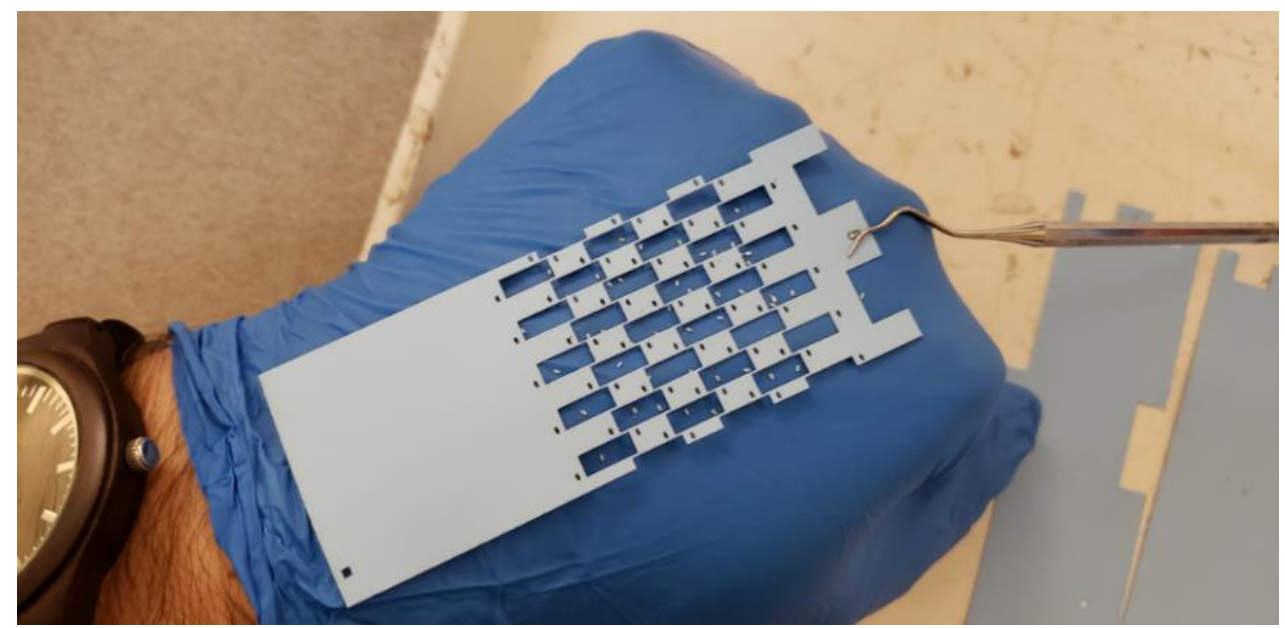

Figure 70. Clearing out the vias

Place the cleaned layers onto a cleaned tray as shown in Figure 71 to get ready for applying paste.

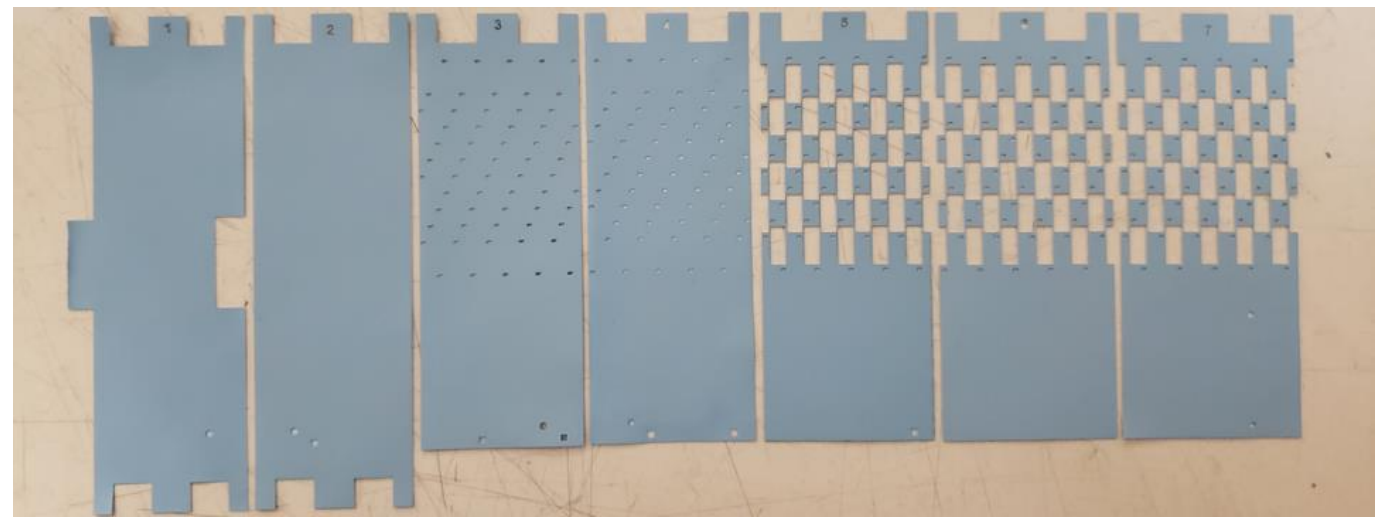

Figure 71. Cleaned layers on a clean tray

\section{$\underline{\text { Paste Deposition }}$}

Locate and turn on the heater and isostatic press to allow them to warm up. The heater (Figure 72a) should be set to whatever temperature is required to dry the paste and the isostatic press (Figure 72b) should be set to $70^{\circ} \mathrm{C}$. 


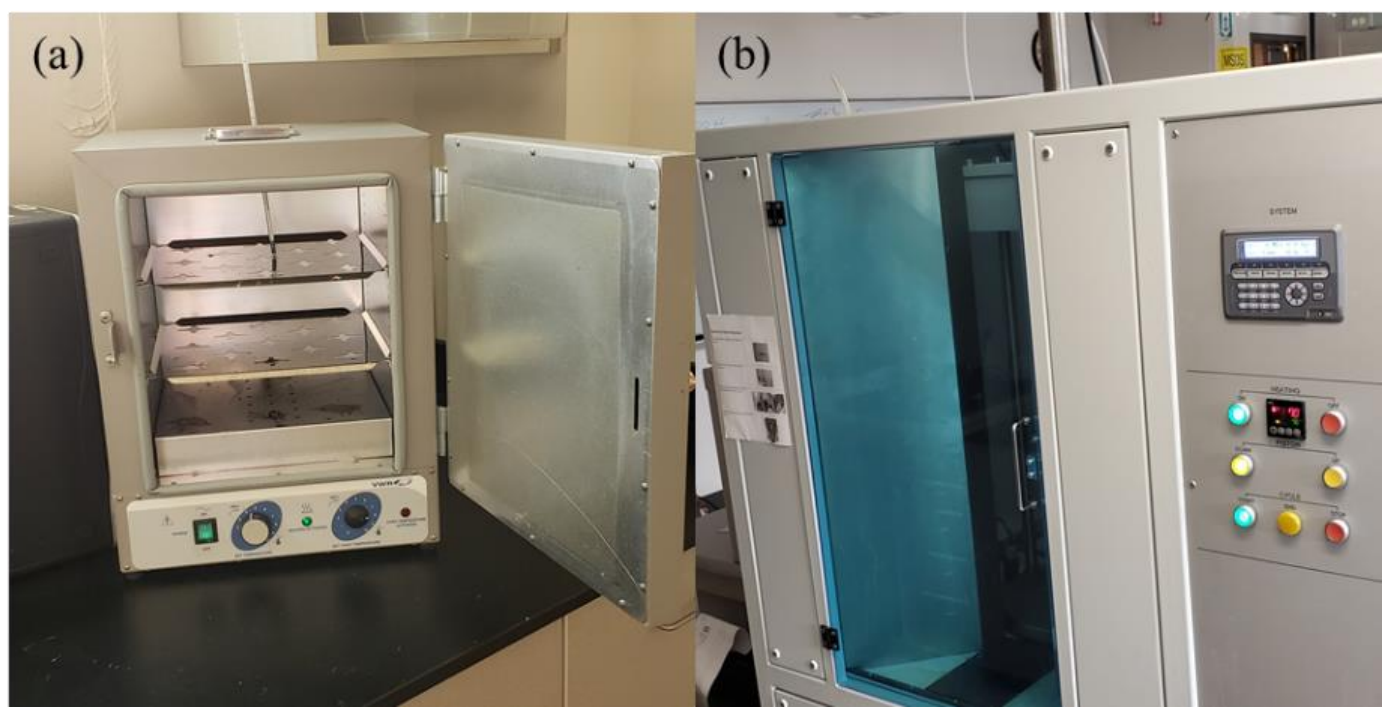

Figure 72. (a) Heater and (b) isostatic press

Locate the items required for screen printing paste to the layers: screen with template already cut, squeegee, silver paste, smooth Teflon taped metal block, scotch tape, and the desired layers shown in Figure 73.

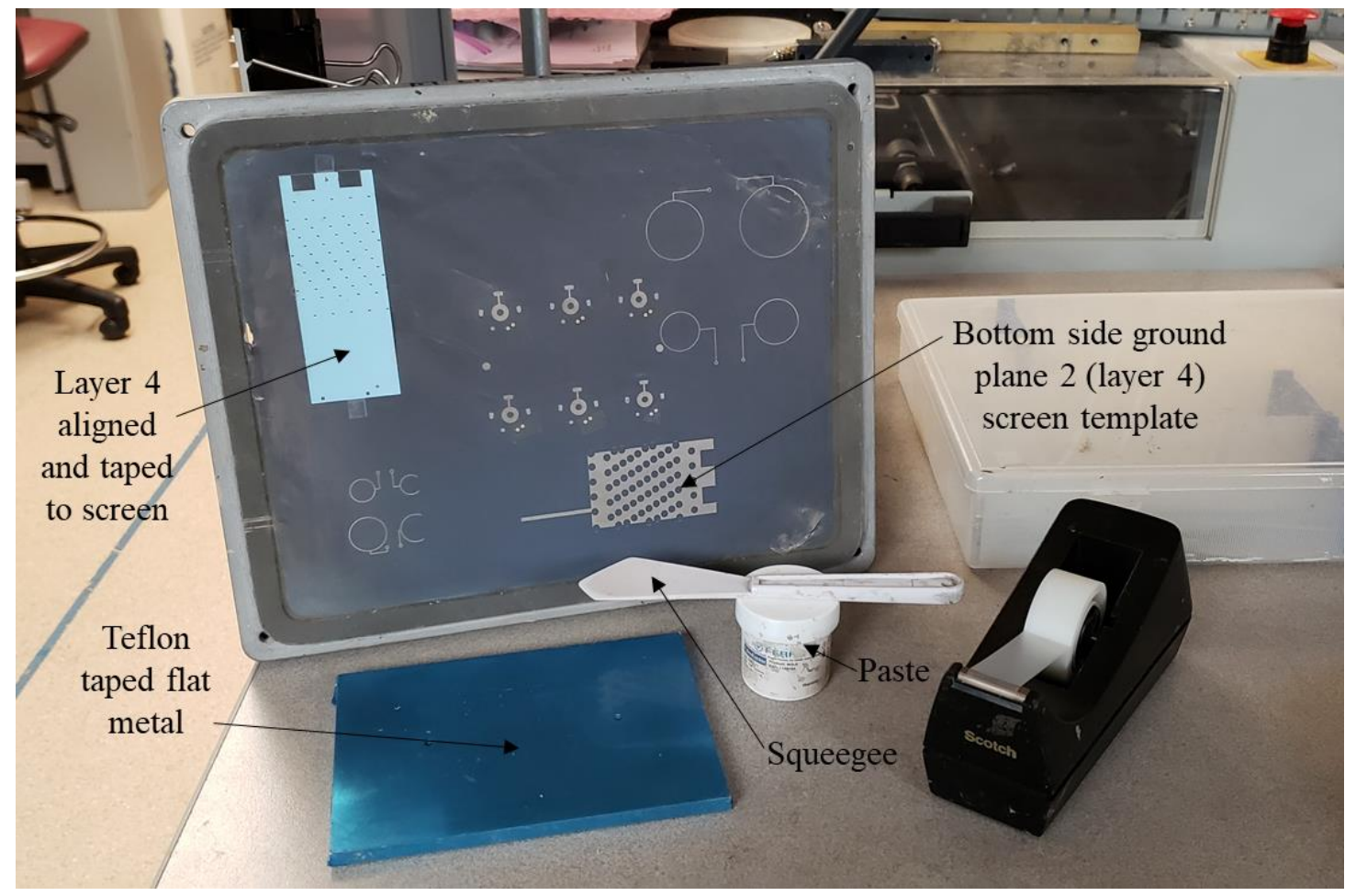

Figure 73. Supplies for Screen printing 
Align the top (matte blue) of layer 4 vias with the holes on the bottom side (dark blue emulsion) of the screen and tape down, shown in Figure 73. The top side (mesh) of the screen aligned with the top of layer 4 is shown in Figure 74a. Scoop paste from the paste container using the squeegee and squeegee the paste across the mesh side of the screen onto layer 4 . Once the layer is covered in paste, gently remove the tape and peel off layer 4 from the screen. Immediately, spray the screen with isopropyl alcohol $\left(\mathrm{C}_{3} \mathrm{H}_{8} \mathrm{O}\right.$ or IPA) and wipe using an absorbent rag or paper towel on both sides of the screen. Spray again and wipe again with a clean cloth and repeat until the screen is visibly clear. Place layer 4 inside the heater, paste side up for about 5 min or until dry. Repeat this process again to apply the stripline of paste on the top of layer 2, the jumper layer on the bottom of layer 3 , and the ground plane paste on the bottom of layer 1 . Figure $74 \mathrm{~b}$ shows the paste layers applied to the bottom of layer 1 and top of layers 2 and 4 . The actual geometry of the stripline (layer 2), jumper (layer 3 not shown), and ground planes (layers 1 and 4) may be different from the examples shown, but the alignment of vias to holes, sides of paste applied, and paste to edges, should remain the same. 


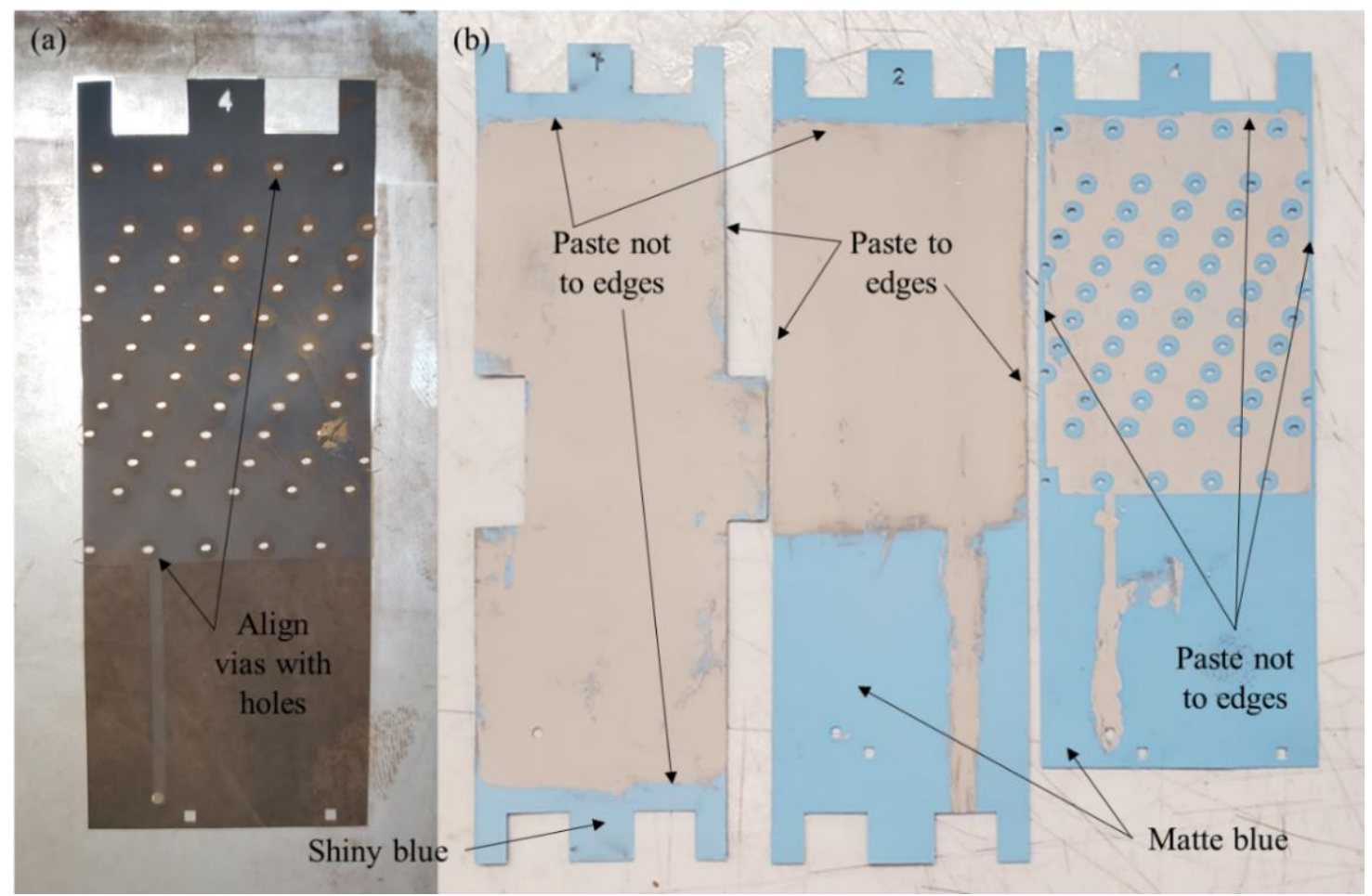

Figure 74. (a) Example layer 4 and (b) layers with paste

After all of the layers have been screen printed (layers 1 to 4 ), the next step is to fill all of the vias. In addition to the same materials as screen printing, locate the 8 ' $x 8$ ' white mylar paper sheets and the plastic via templates (3 to 7). Cut the paper into four squarish pieces and place one on the Teflon block with the non-sticky side up. Use a tape test to identify the non-sticky side. Next, place layer 3 on top of the mylar paper with the top of layer 3 facing up. Place the layer 3 template on top of layer 3 and align the vias, shown in Figure 75a. Then squeegee paste across the template and into the via holes shown in Figure 75b. Carefully remove the template without moving the layer or paper shown in Figure 75c. Slide the paper and layer off of the block and set aside. Repeat this process for layer 5, 6, and 7. Since the vias will shrink in the firing process, filling the layer 4 vias is NOT required and helps prevent shorting. The paste in layer 3 and 5 vias will push and fill the gap. 


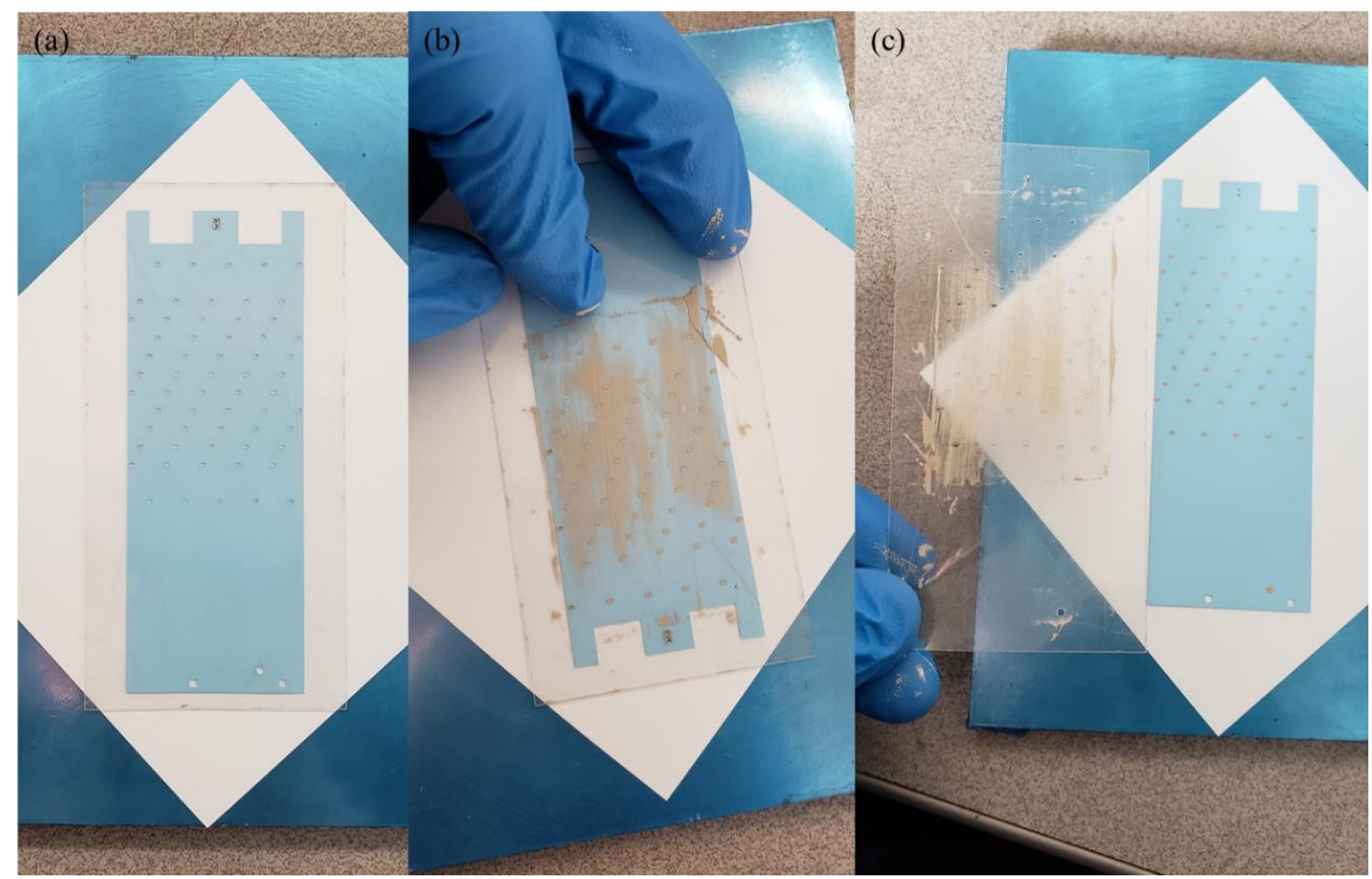

Figure 75. (a) Flat fill alignment, (b) squeegeeing, and (c) removal

Place all four via filled layers $(3,5,6$, and 7$)$ into the heater for $30 \mathrm{~min}$ at $70^{\circ} \mathrm{C}$ if using Ferro $903-\mathrm{A}, 10 \mathrm{~min}$ at $110^{\circ} \mathrm{C}$ if using DuPont 6145 , or $5 \mathrm{~min}$ at $110^{\circ} \mathrm{C}$ if using DuPont 6141 as shown in Figure 76a. After the first drying period, gently remove the layers, flip them over and roll the paper off, to avoid removing the filled vias as shown in Figure 76b. Place the layers back into the heater for another $5 \mathrm{~min}$ at the same temperature, as shown in Figure 76c. 


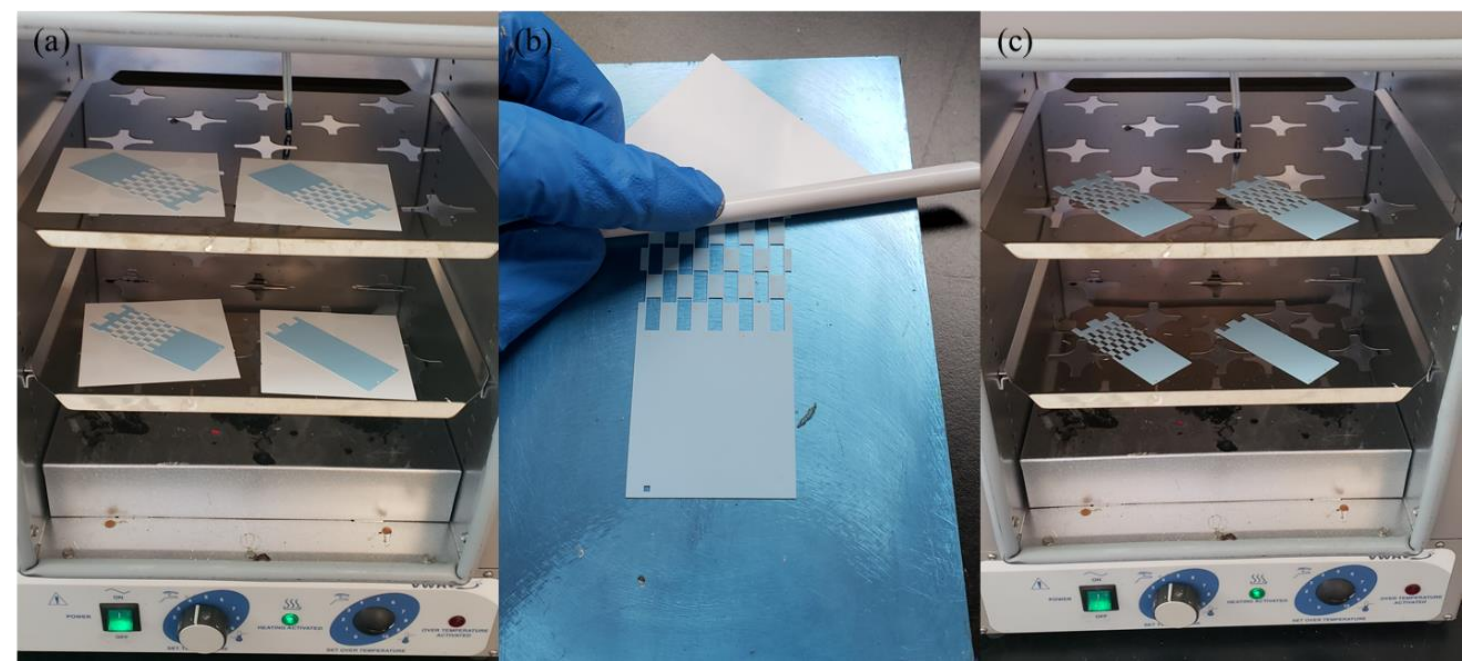

\section{Figure 76. (a) Drying with paper, (b) paper removal, and (c) drying again}

Using a similar process as the flat fill method above, fill the larger circular ground vias on the bottom of layers 1,2,3, and 4. Make sure that the ground via on layer 2 does not contact the stripline in anyway. The layer 3 ground via should have already been filled when using the plastic via template. Also, fill the two-facet plate vias on layer 7 which should have already been filled with the plastic via template.

Before moving on, make sure that all of the silver paste has been applied. Layer 1 should have a ground plane layer on the bottom that connects to a ground circular via on the lower part of the layer. Layer 2 should have a stripline layer on the top (will differ from Figure 77) and a ground circular via that DOES NOT connect to the stripline. Layer 3 should have 60 filled vias, a jumper layer on the bottom, and a ground circular via that DOES NOT connect to the jumper or vias. Layer 4 should have a ground plane on the top and a ground circular via that DOES connect to the ground and NO filled vias. Layers 5 and 6 should have 60 filled vias each. Finally, Layer 7 should have 60 filled vias, two filled facet plate vias, and a facet plate connection layer that connects the two vias. Figure 77 shows a top view of all seven layers cleaned and with paste. 


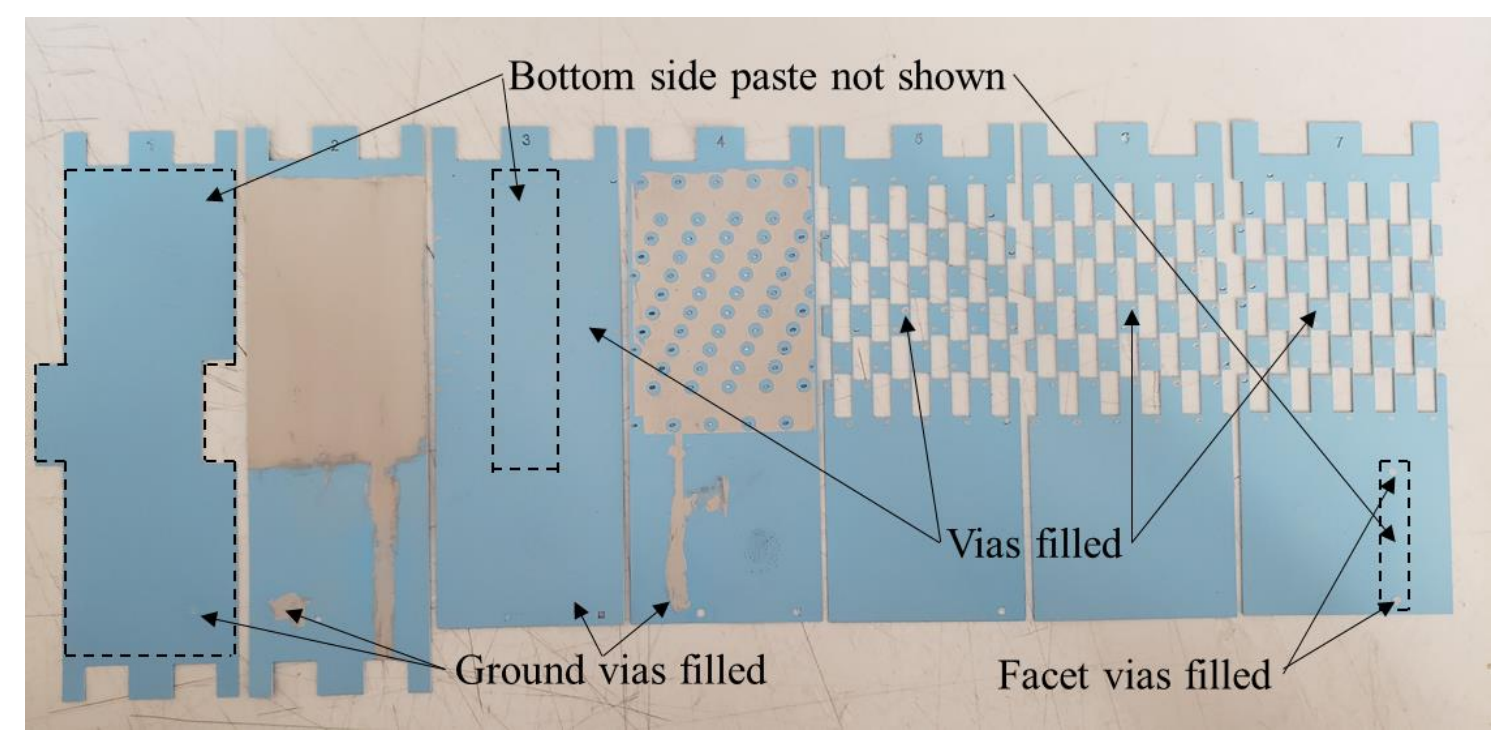

Figure 77. Top view of all seven completed layers

Wrapping

Locate an 8'x8' mylar sheet and place it on the table non-sticky side up. Place layer 4 on the sheet with the bottom side up (silver paste ground facing down). Apply PEOX on the left side of layer 4 and using the alignment tabs and fiducial marks, lay layer 3 on top of layer 4, bottom of layer 3 facing up. The middle of layer 3 should be glued to the left edge of layer 4. Applying too much pressure to the PEOX can risk breaking layer 3 in half. Apply PEOX on the left side of layer 3 and using the alignment tabs and fiducial marks, lay layer 2 on top of layer 3, bottom of layer 2 facing up (silver paste stripline facing down). Apply PEOX on the left side of layer 2 and using the alignment tabs, lay layer 1 on top of layer 2, bottom of layer 1 facing up (silver paste ground facing up). This process is shown in Figure 78a and the top side of layers 1 to 4 are shown in Figure 78b. 


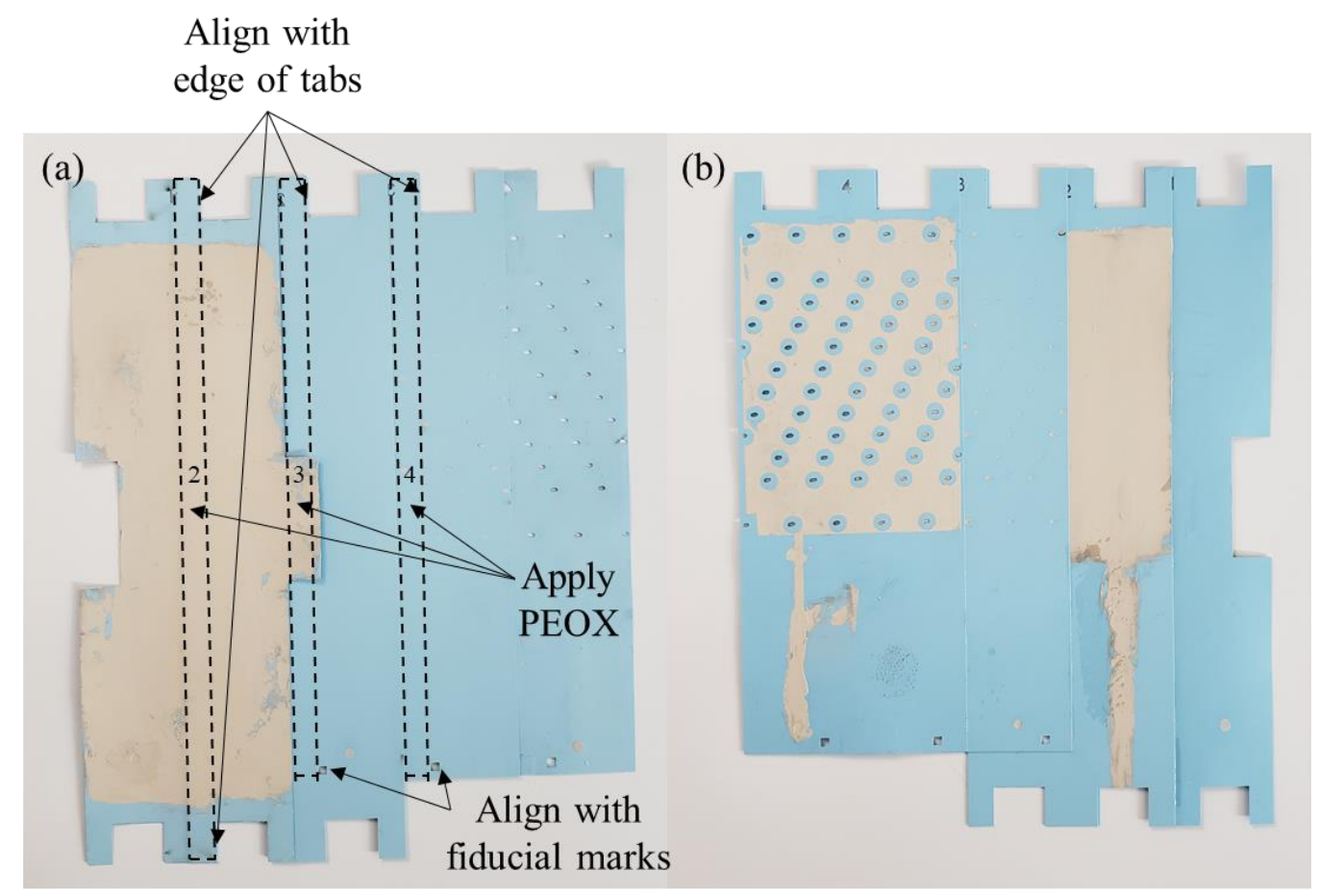

Figure 78. (a) Bottom side and (b) top side of layer by group

Locate the jig and wax paper (Figure 79a), cut the wax paper to the length of the jig and to the width of two wraps of the jig. Using just enough tape to hold the wax paper, tape the first edge of the paper to the jig, tightly wrap the paper around twice and tape down. This is shown in Figure 79a. Lay the glued layers 1 to 4 bottom side up and place the jig on layer 1, shown in Figure 79b. Wrap the layers together on the jig and keep rolling until the layer 4 edges meet, shown in Figure 79c (paste removed to show edges meeting). Once the layer 4 edges meet, carefully peel back layer 4 and apply just enough PEOX to hold the layers tightly together. 


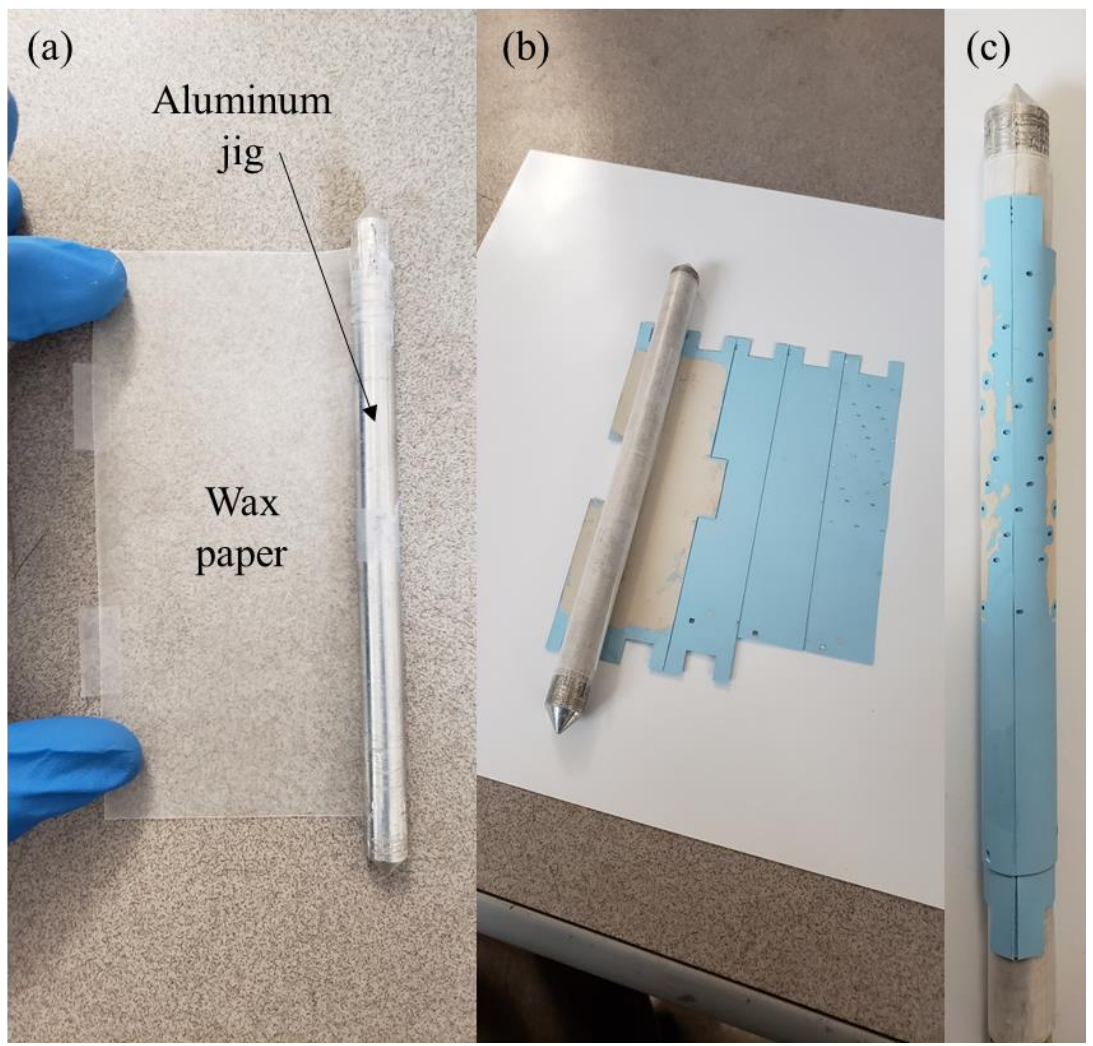

Figure 79. (a) Jig preparation, (b) jig wrapping, and (c) rolled layer 1 to 4

Rotate the jig so that the layer 4 number and fiducial mark faces up (Figure 80a).

Place layer 5 top side down on the mylar paper, apply PEOX to the far-left side and place on top of layer 4 using the alignment tabs and fiducial marks to align (Figure 80b, example layer 5 shown). Rotate the jig and layer so that layer 5 is on the bottom and apply PEOX to the right edge of layer 5 (Figure 80c, example layer 5 shown). Roll the jig once (max twice or else layer 5 will break) so that the edges of layer 5 meet (Figure 80d, actual layer 5 shown). The other side of actual layer 5 is shown in Figure 80e. Notice that the layer 4 ground paste layer almost touches but does not, the silver paint used to adhere the die will complete the connection across the edges. Once layer 5 is wrapped, laminate the jig and layer (see laminating section). The small amount of PEOX used will allow the layers to shift and fall into place if they aren't already. 


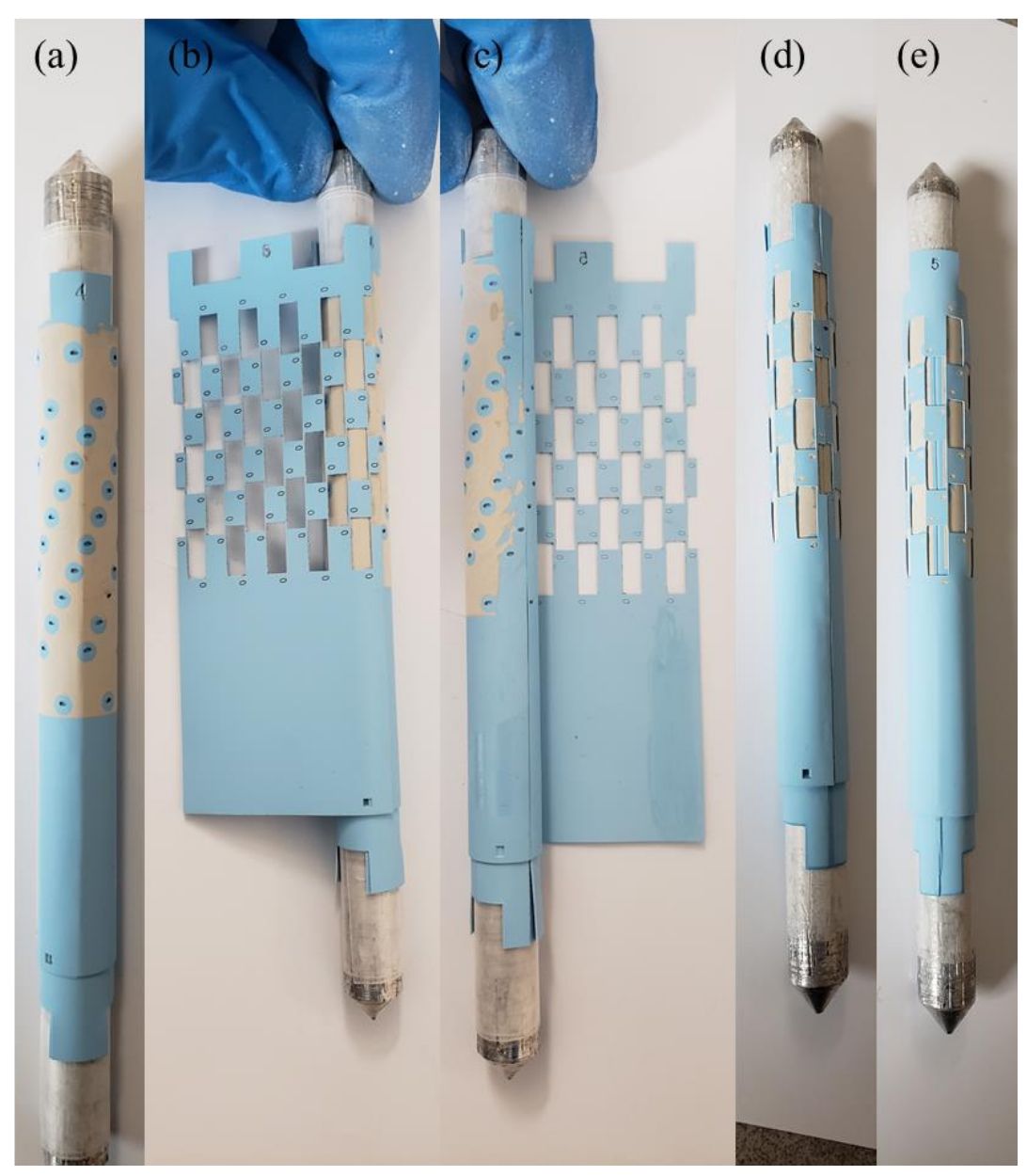

\section{Figure 80. (a) Lay flat, (b) align layer 5, (c) flip over, (d) roll, (e) final}

After lamination (Figure 81a), PEOX layer 6 to layer 5 using the rectangles to align (Figure 81b). Make sure the seams of layer 6 line up with the middle of layer 5, roll, PEOX, and then repeat for layer 7. Orient the jig and layers so that the seams of layer 7 faces up and apply PEOX to the spacer strips shown in Figure 81c. Place the middle of the strips on the seam and wrap around layer 7 (Figure 81d). The fully wrapped cathode is shown in Figure 81e. 

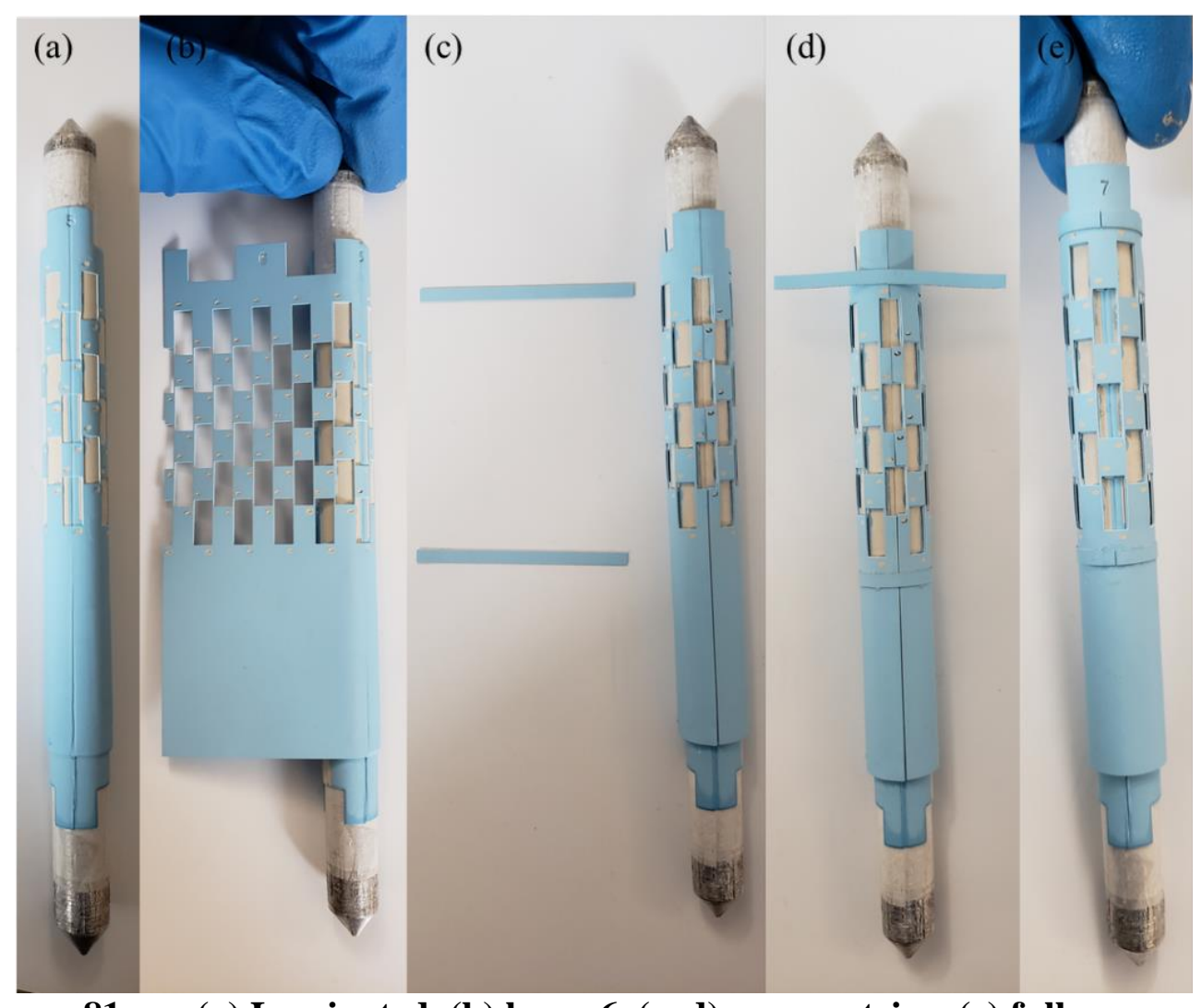

Figure 81.

\section{(a) Laminated, (b) layer 6, (c, d) spacer strips, (e) fully wrapped cathode}

\section{$\underline{\text { Laminating }}$}

Once the jig is ready to be pressed, the jig and layers are placed in a vacuum bag (Figure 82a) which gets placed in the vacuum that seals the bag (Figure 82b). After the bag has been sealed, it gets placed in to the isostatic press (Figure 82c) where it is pressed under $10.35 \mathrm{MPa}(1.5 \mathrm{ksi})$ at $70^{\circ} \mathrm{C}\left(158^{\circ} \mathrm{F}\right)$ for $8 \mathrm{~min}$ (Figure $\left.82 \mathrm{~d}\right)$. Occasionally the bags will be a bad batch and leak under pressure which ruins the cathode. The solution is to either use two of the bad bags, or to use a different batch. The new bags are thicker than usual (7 mil) and have not leaked thus far. After lamination, cut open the vacuum bag, remove the cathode and jig, and discard the bag. 


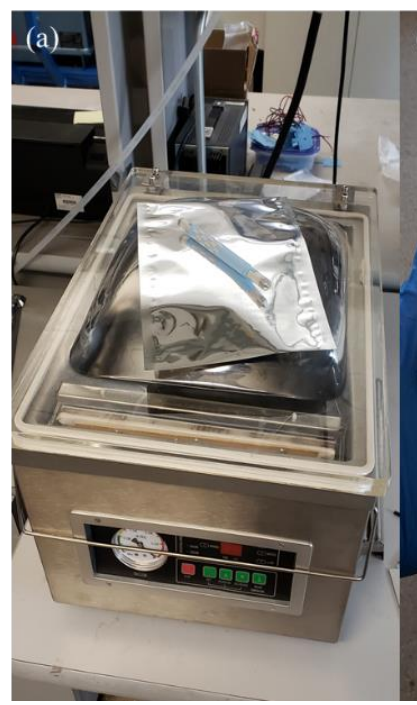

Figure 82.
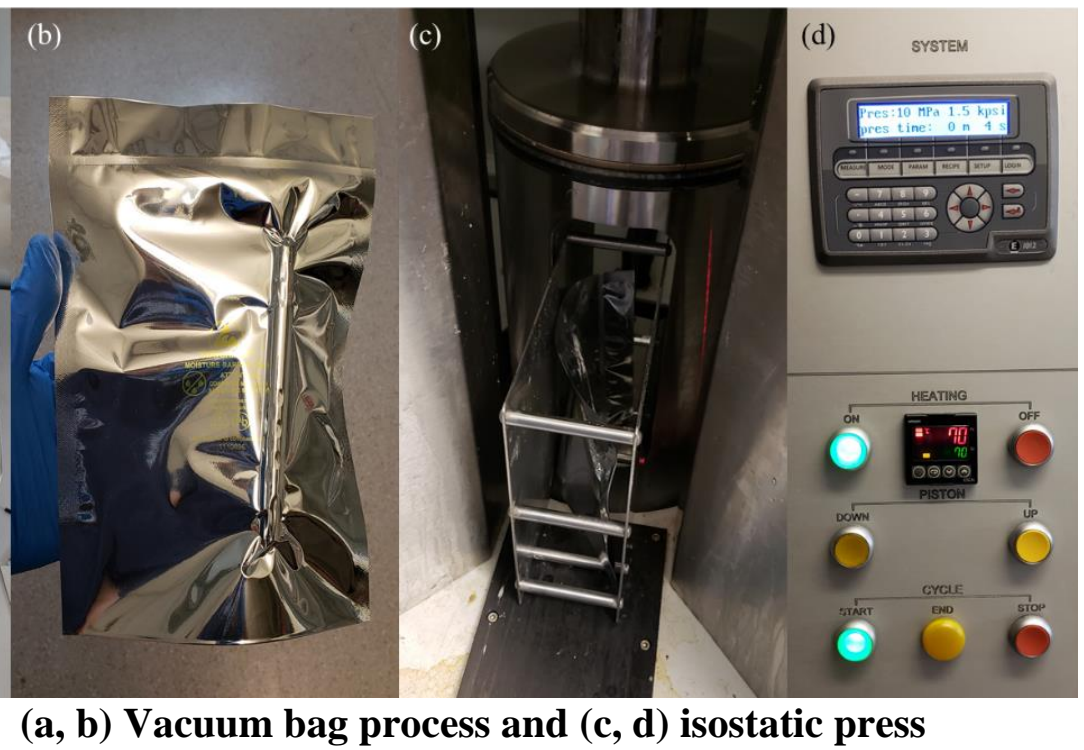

$(a, b)$ Vacuum bag process and $(c, d)$ isostatic press

Figure 83a and Figure 83c shows the vacuum bag and cathode before lamination respectively. Figure $83 \mathrm{~b}$ and Figure $83 \mathrm{c}$ shows the vacuum bag and cathode after lamination respectively.

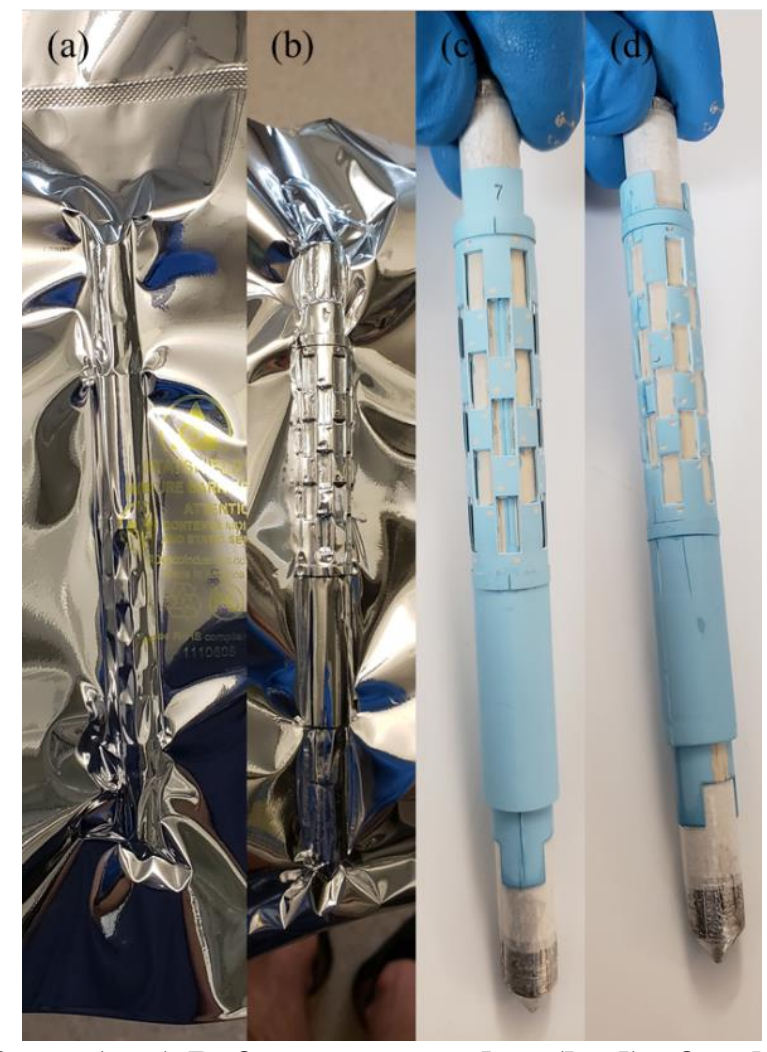

Figure 83. (a, c) Before compared to $(b, d)$ after lamination 
Firing

After final lamination, the cathode is ready to be removed from the jig. Using a razor or pizza cutter, cut the alignment tabs from the top and bottom of the cathode (Figure 84a). Using vise grips, clamp the jig and by hand carefully rotate and pull the cathode until it breaks free from the wax paper and slides off the jig (Figure 84b). Using tweezers, grab the remaining wax paper and rotate until it detaches from inside the cathode (Figure 84c). Finally, place the cathode on the firing stand upside down (rectangles closer to the bottom) inside the furnace (Figure 84d). Occasionally spray the stand with boron nitride to prevent the LTCC from sticking while being fired.

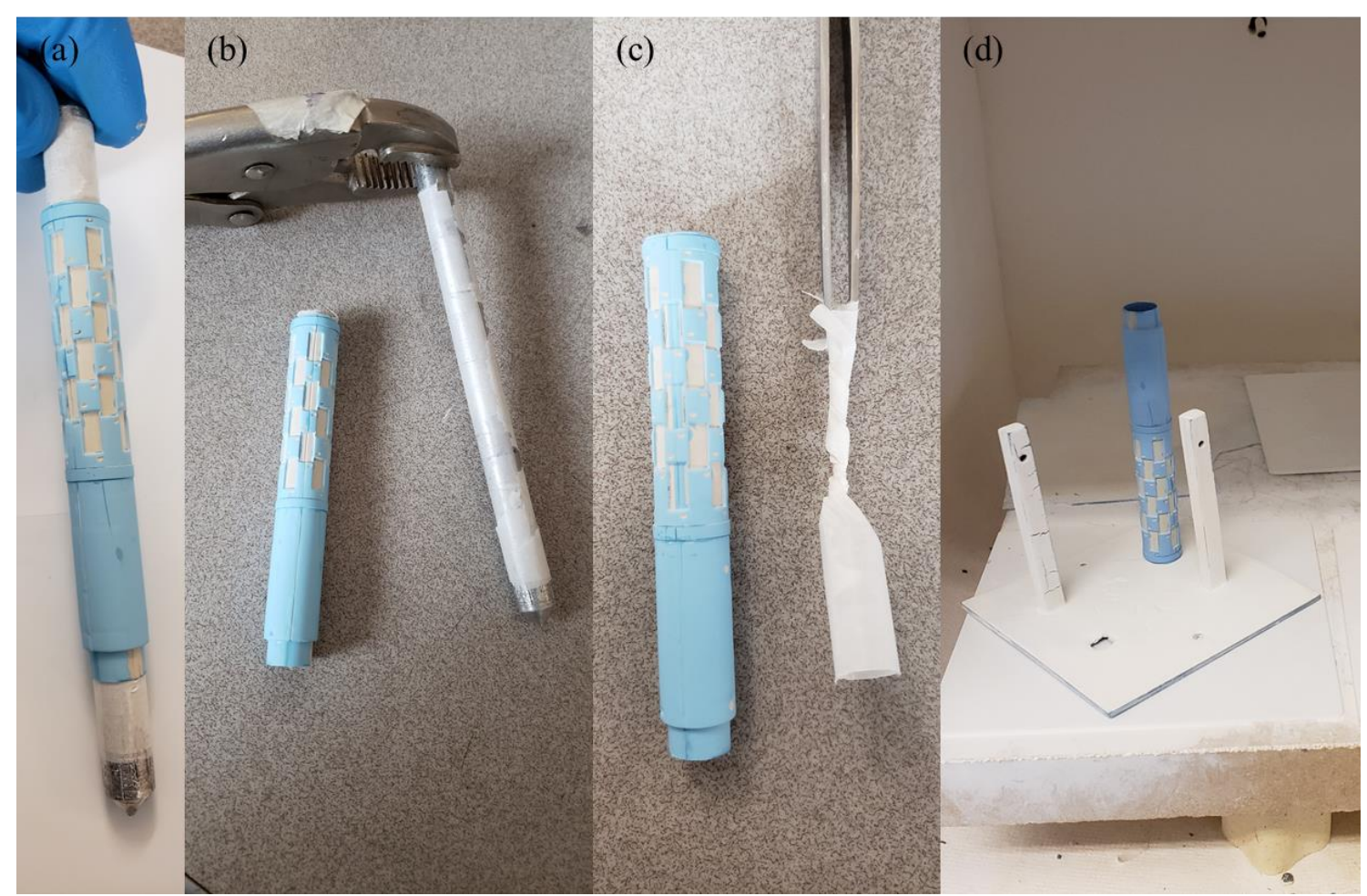

Figure 84. (a) Removing tabs, (b) cathode, (c) wax paper, and (d) placing on stand

Set the Furnace firing profile to the one shown in Figure 85 and hit 'RUN.' 


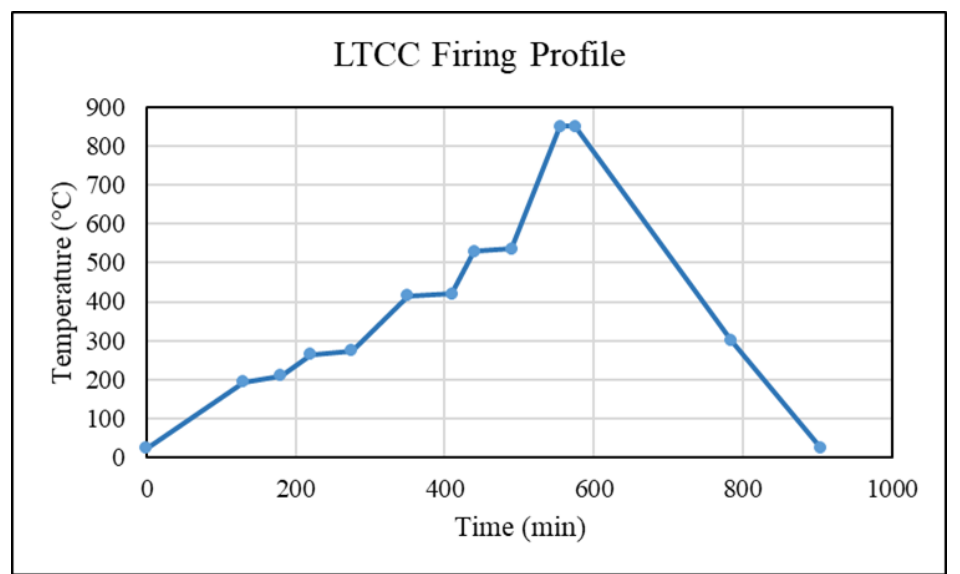

Figure 85. LTCC system firing temperature profile

\section{$\underline{\text { Facet Plates }}$}

The last components of the cathode are the facet plates. First, start by using the laser cutter to cut out five rectangular sheets of 10-mil LTCC (Figure 86a). Clean and then press all five sheets together using the axial press at $20.7 \mathrm{MPa}(3 \mathrm{ksi})$ at $70^{\circ} \mathrm{C}$ $\left(158^{\circ} \mathrm{F}\right)$ for $8 \mathrm{~min}$ (Figure $\left.86 \mathrm{~b}\right)$. Remove the laminated block of LTCC from the press and tape it to a millable substrate (usually clear plastic). Tape the LTCC block and substrate to the vacuum bed on the Bungard PCB mill (Figure 86c).

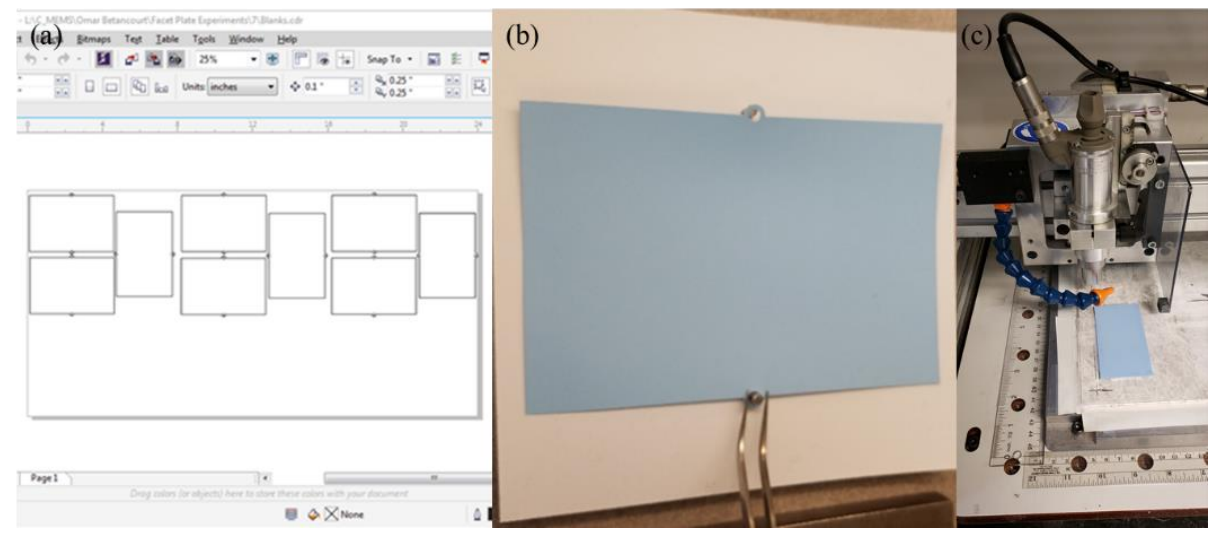

Figure 86. (a) Rectangular sheets, (b) lamination, and (c) fixturing to mill

Open the desired mill pattern in CorelDraw, place the cutting pattern in the center of the page and save as a 'PLT' file. Transfer and save the file onto the desktop of the 
computer by the mill. Locate and install the $60^{\circ}$ ' $\mathrm{V}$ ' shaped end mill into the collet of the PCB mill. Turn on the mill and open the 'ROUTERPRO' software to control the Bungard PCB mill. The main menu (Figure 87b) will popup. Load the desired slit file (just cuts the slits of the facet plates) in the 'Route file' section. Click 'Offset' (Figure 87d) and move the tool until it is centered with the block of LTCC, keep the ' $Z$ ' position at $30 \mathrm{~mm}$, do NOT adjust. Click 'OK' to accept and then 'View/Hide' or 'View/Hide Table' until the pattern is shown in relation to the bed (Figure 87a). Use this to verify that the offset (pattern location) is correct. Go back to the main menu and click 'Tools' (Figure 87c), change the T1 Depth $(\mathrm{mm})$ and Iter $(/ \mathrm{n})$ to 0 and 1 respectively. Click 'Update', then 'OK'. Leave the 'Base' at 21.462 and change the 'Board' to 8 and then click 'Update Data'. Click 'Start Routing', 'Play' (Figure 87e), 'Tool is inserted' (Figure 87f) , and then 'Start Spindle' (Figure 87g) to start the milling process (Figure 87h). Keep lowering the 'Board' number until the end mill just barely scrapes the top of the LTCC block. Once it does, lower the 'Board' number again by 0.03 . Go back to 'Tools' and change the 'Depth' to $1.2 \mathrm{~mm}$, 'Iter' to 24 , ' $\mathrm{X}$ and $\mathrm{Z}$ feed' to $250 \mathrm{~mm} / \mathrm{min}$, and 'Spindle' to 40,000 1/min. Click 'Update', 'OK', and then 'Start Routing'. After it finishes, the slits should be cut out of the facet plates. Next, replace the $60^{\circ}$ ' $\mathrm{V}$ ' shaped end mill with the $40^{\circ}$ ' $\mathrm{V}$ ' shaped end mill. Load the desired cutout file (just cuts the outside of the facet plates) that matches the slit file previously used. Keep the SAME offset and change the T1 Depth and Iter back to 0 and 1 respectively. Recalibrate the 'Board' number until the end mill just barely scrapes the top of the LTCC block. Once it does, change the T1 Depth and Iter back to 1.2 and 24 respectively. Click 'Start Routing' to start cutting the outside of the facet plates and the final cut. 


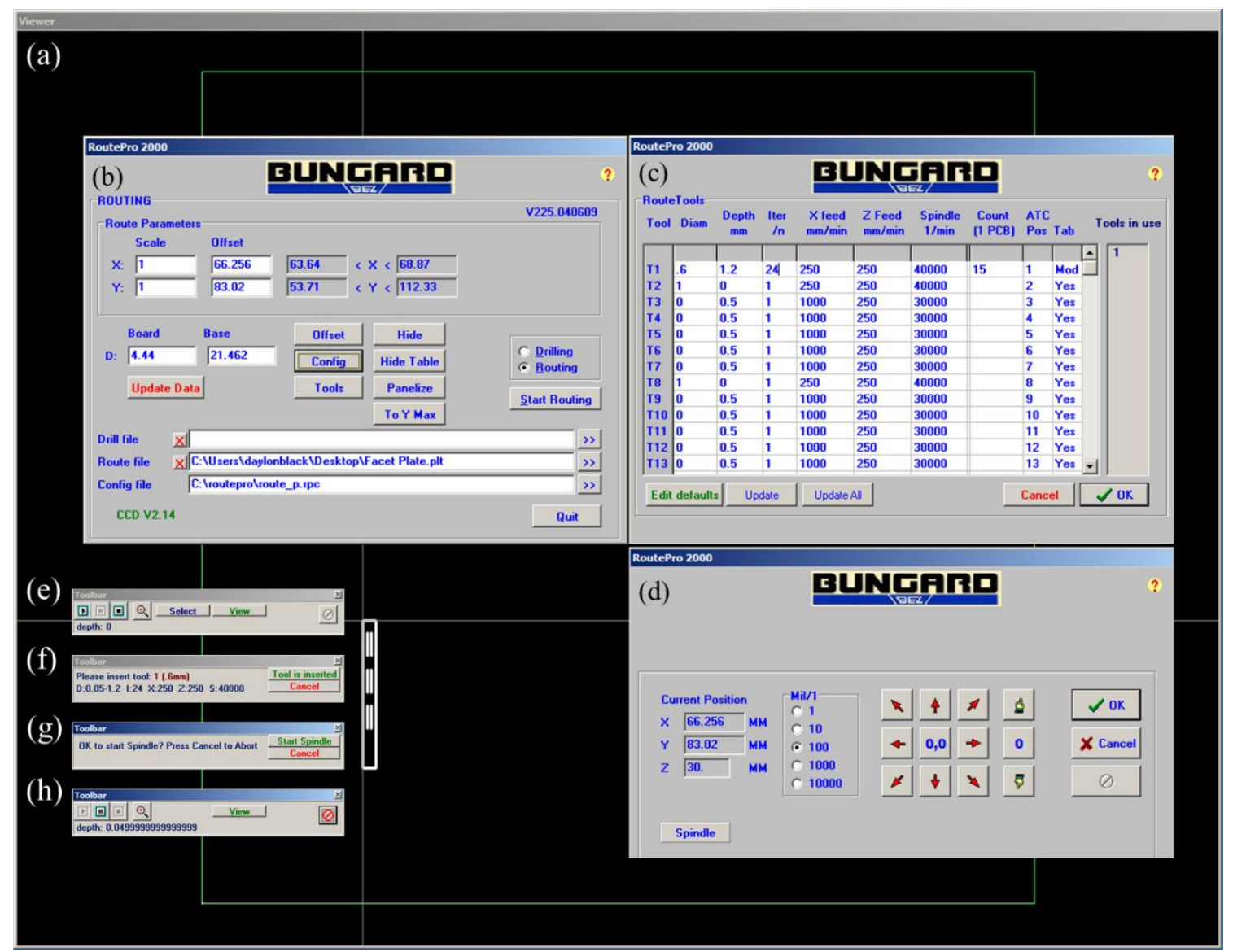

Figure 87. ROUTRPRO software

Once milling is complete, run compressed air across the surface to clean off any left-over debris (Figure 88a). Remove the facet plates and hold up to light to verify the slits are completely through the substrate. Also use a microscope to verify that a point has formed in between the two slits (Figure 88b). Once geometry has been verified, place the facet plates in the furnace to be fired at the same profile and temperatures as the cathode. 


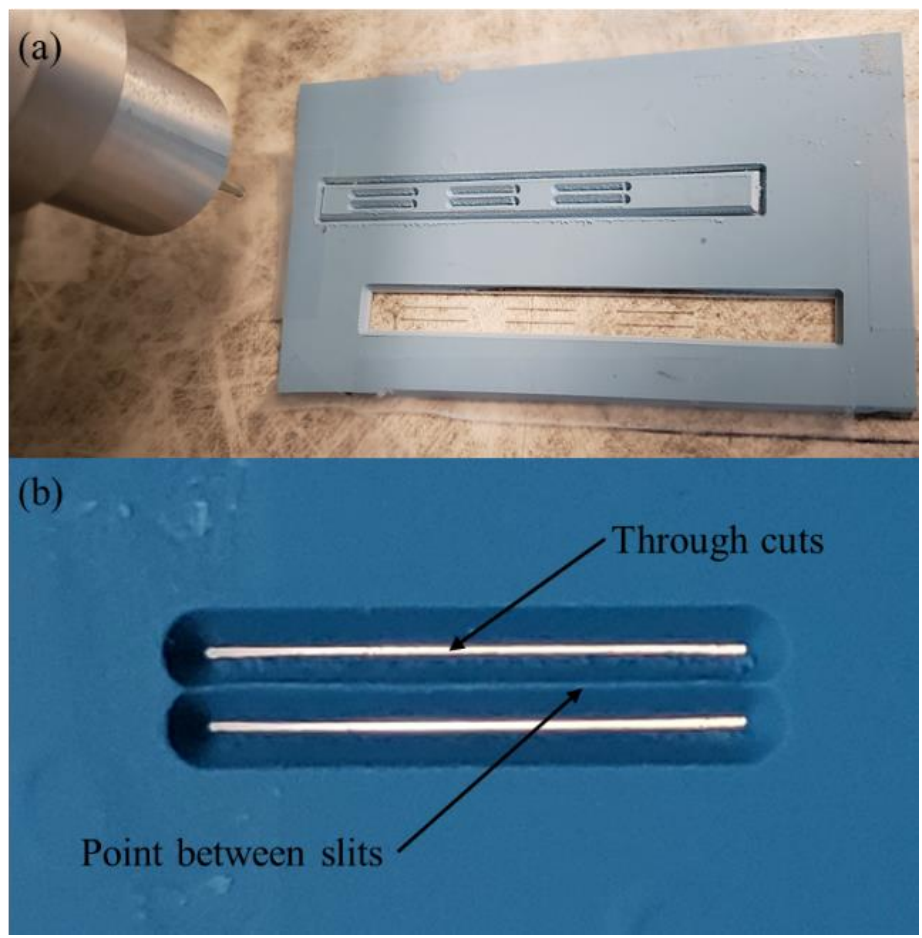

Figure 88. (a) Green facet plate and (b) fired slits showing verifications 
APPENDIX B: CATHODE TRIALS 


\begin{tabular}{|c|c|c|c|c|c|c|c|c|c|c|c|}
\hline \multicolumn{3}{|c|}{ Cathode } & \multicolumn{4}{|c|}{ Paste } & \multicolumn{3}{|c|}{ Build } & \multirow[b]{2}{*}{ Goal } & \multirow[b]{2}{*}{ notes } \\
\hline Name & LTCC & Layers & Via & 1st Ground & 4th Ground & 2nd Trace & Wrap & Press & Fire & & \\
\hline a & DuPont 951PX \& 951PT & $1,2,3,4,5,6,7$ & & & & & 0 & & 1 & 1 & 0 wrap process slow and very difficult, requires practice \\
\hline$b$ & DuPont 951PX \& 951PT & $1,2,3,4,5,6,7$ & & & & & 0 & & 1 & 1 & 0 \\
\hline c & DuPont 951PX \& 951PT & $1,2,3,4,5,6,7$ & & & & & 0 & & 1 & 1 & 0 \\
\hline DLDR1 & DuPont 951PX \& 951PT & $1,2,3,4,5,6,7$ & & 1 DuPont 6145 & DuPont 6145 & DuPont 6145 & 1 & & 1 & 1 & 1 shorted, $3 \mathrm{~mm}$ grnd holes \\
\hline DLDR2 & DuPont 951PX \& 951PT & $1,2,3,4,5,6,7$ & & 1 DuPont 6145 & DuPont 6145 & DuPont 6145 & 1 & & 2 & 1 & 1 shorted, broke during wrap, did not fire \\
\hline DLDR3 & DuPont 951PX \& 951PT & $1,2,3,4,5$ & & 1 DuPont 6145 & DuPont 6145 & DuPont 6145 & 1 & & 2 & 1 & 1 not shorted! Yay \\
\hline DLDR4 & DuPont 951PX \& 951PT & $1,2,3,4,5,6,7$ & & 2 DuPont 5081 & DuPont 5081 & DuPont 5081 & 2 & & 2 & 1 & 1 shorted \\
\hline DLDR5 & DuPont 951PX \& 951PT & $1,2,3,4,5,6,7$ & & 2 DuPont 5081 & DuPont 5081 & DuPont 5081 & 2 & & 2 & 1 & 1 shorted \\
\hline MONEY1 & DuPont 951PX \& 951PT & $1,2,3,4,5,6,7$ & & 2 DuPont 5081 & DuPont 5081 & DuPont 5081 & 3 & & 2 & 1 & 1 not shorted! Yay, 4mm grnd holes \\
\hline MONEY2 & DuPont 951PX \& 951PT & $1,2,3,4,5,6,7$ & & 2 DuPont 5081 & DuPont 5081 & DuPont 5081 & 3 & & 2 & 1 & 1 not shorted! Yay \\
\hline MONEY3 & DuPont 951PX \& 951PT & $1,2,3,4,5,6,7$ & & 2 DuPont 5081 & DuPont 5081 & DuPont 5081 & 3 & & 2 & 1 & 1 not shorted, $6 \& 7$ layers broke during wrap \\
\hline NO4P1 & DuPont 951PX \& 951PT & $1,2,3,4,5$ & & 2 & & DuPont 5081 & 3 & & 2 & 1 & 20 not connected \\
\hline NO4P2 & DuPont 951PX \& 951PT & $1,2,3,4,5$ & & 2 & & DuPont 5081 & 3 & 3 & 2 & 1 & 21 not connected \\
\hline FatTrace & DuPont 951PX \& 951PT & $1,2,3,4$ & & 2 & & DuPont 5081 & 3 & 3 & 2 & 1 & 3 Bad Wrap,L2 overlapped/did not meet normally, not connected \\
\hline Skinny & DuPont 951PX \& 951PT & $1,2,3,4$ & & 2 & & DuPont 5081 & 3 & 3 & 2 & 1 & 3 connected \\
\hline Jumper & DuPont 951PX \& 951PT & $1,2,3,4$ & & 2 & & DuPont 5081 & 3 & 3 & 2 & 1 & 3 connected, jumper on L3 probs best \\
\hline Trace1 & DuPont 951PX \& 951PT & $1,2,3,4$ & & 2 & & ESL 903-A & 3 & 3 & 2 & 1 & 4 a few trace lines connected \\
\hline Trace2 & DuPont 951PX \& 951PT & $1,2,3,4$ & & 2 & & ESL 903-A & 3 & 3 & 2 & 1 & 4 a few connected, requires perfect paste and perfect wrap \\
\hline Trace3 & DuPont 951PX \& 951PT & $1,2,3,4$ & & & & ESL 903-A & 3 & 3 & 2 & 1 & 5 no vias, inconclusive \\
\hline Trace4 & DuPont 951PX \& 951PT & $1,2,3,4$ & & & & ESL 903-A & 3 & 3 & 2 & 1 & 5 did not fire \\
\hline Trace5 & DuPont 951PX \& 951PT & $1,2,3,4$ & & 2 & & Ferro 903-A & 3 & 3 & 2 & 1 & 5 connected! \\
\hline Trace6 & DuPont 951PX \& 951PT & $1,2,3,4$ & & 2 & & Ferro 903-A & 3 & 3 & 2 & 1 & 5 Most are connected, visibly not connected to main \\
\hline Trace7 & DuPont 951PX \& 951PT & $1,2,3,4$ & & 2 & & Ferro 903-A & 3 & 3 & 2 & 1 & 6 Both ends are not connected \\
\hline MONEY4 & DuPont 951PX \& 951PT & $1,2,3,4,5,6,7$ & & 2 Ferro 903-A & Ferro 903-A & Ferro 903-A & 3 & 3 & 2 & 1 & 1 not shorted, more circular than others cause new paste \\
\hline Pump1 & DuPont 951PX \& 951PT & $1,2,3,4,5,6,7$ & & 3 & Ferro 903-A & Ferro 903-A & 3 & 3 & 2 & 1 & 7 not shorted, two vias not connected from PEOX \\
\hline Pump2 & DuPont 951PX \& 951PT & $1,2,3,4,5,6,7$ & & 3 & Ferro 903-A & Ferro 903-A & 3 & 3 & 2 & 1 & 7 not shorted, pump method confirmed \\
\hline CMEMS & DuPont 951PX \& 951PT & $1,2,3,4,5,6,7$ & & 1 Ferro 903-A & Ferro 903-A & Ferro 903-A & 3 & 3 & 2 & 1 & Cathode for manufacturing process \\
\hline
\end{tabular}




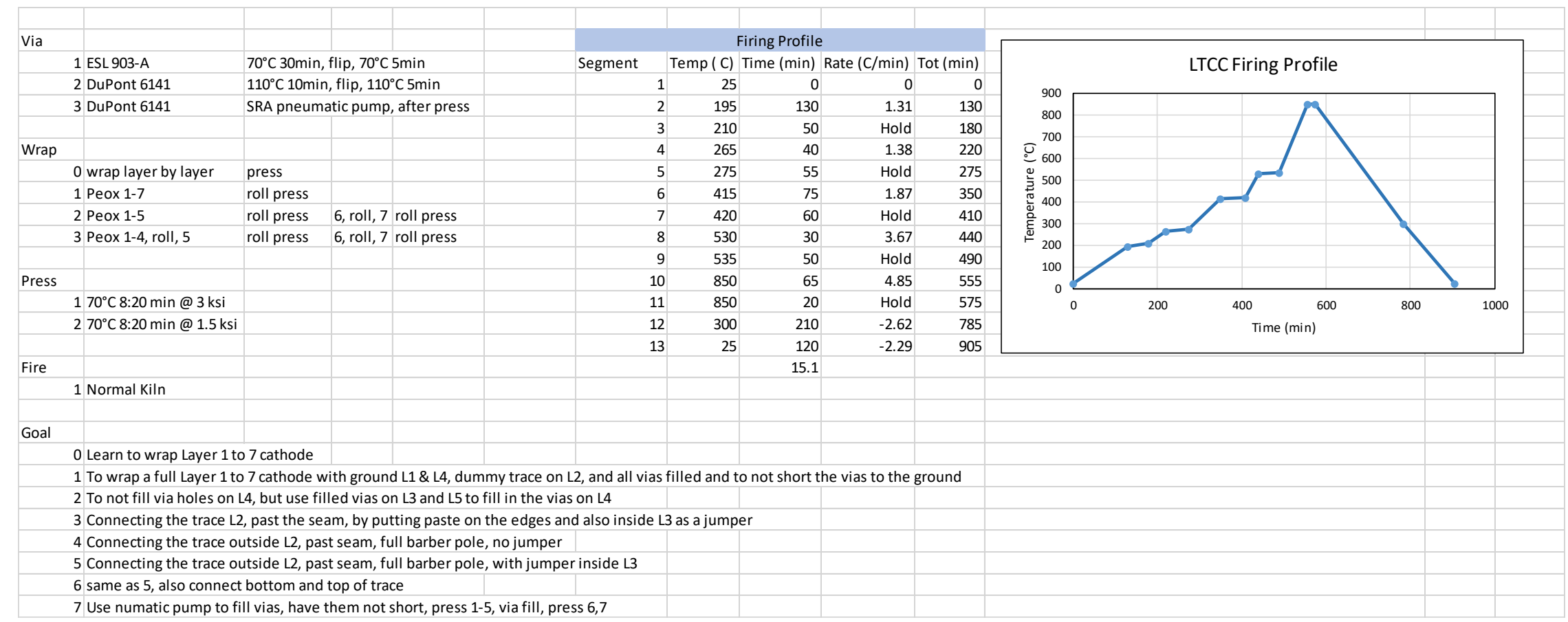


APPENDIX C: FIRING STRUCTURE TRIALS 


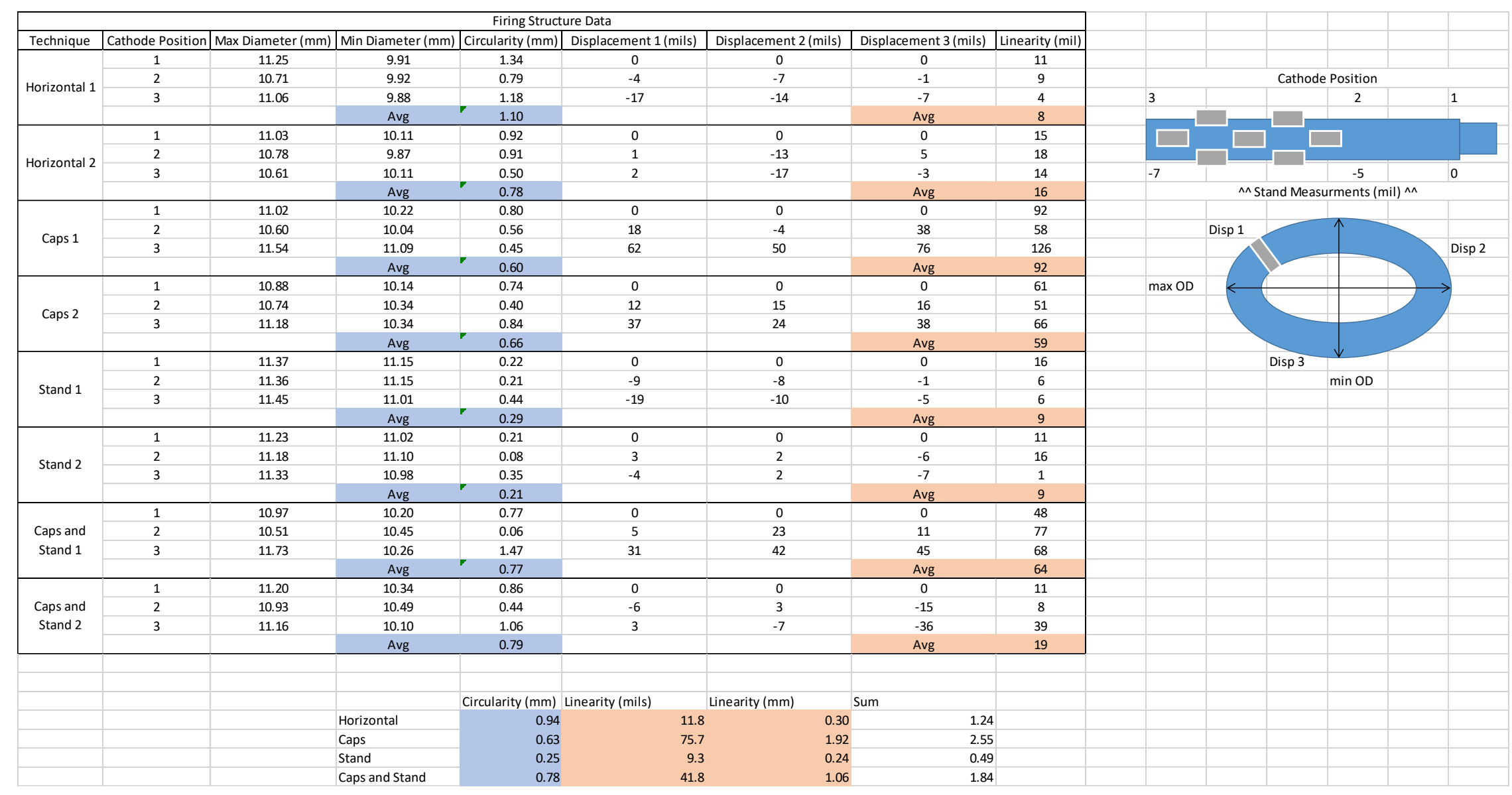


APPENDIX D: RFTF TRIALS 


\begin{tabular}{|c|c|c|c|c|c|c|c|c|c|c|c|c|c|c|c|}
\hline \multirow[b]{2}{*}{ Row } & \multicolumn{3}{|c|}{ RFTF } & \multicolumn{7}{|c|}{ Screen Cutting } & \multicolumn{5}{|c|}{ Silver Paste } \\
\hline & Trial & Name & Model & $Z$ height (in) & $\begin{array}{l}\text { Trace Width } \\
\text { Screen (um) }\end{array}$ & Trace Width Unfired (um) & LTCC Tape & Power (\%) & Speed (\%) & PPI (\%) & Ground Paste & Trace Paste & Ground Pads & s Via & Die \\
\hline 1 & $1 a-23 a$ & & & & & & & & & & & & & & \\
\hline 2 & & $144 * 4$ & RFTF4 & & & & DuPont 951 & & & & & & & & \\
\hline 3 & & 2 ThinOne & RFTF4 & -1.83 & $195 ; 195$ & $194 ; 169 ; 158 ; 162$ & DuPont 951 & 20 & 15 & 1000 & DuPont 6145 & DuPont 6145 & DuPont 6146 & 5 No & No \\
\hline 4 & & 3 ThickOne & RFTF4 & -1.83 & $195 ; 195$ & $237 ; 88 ; 194 ; 195 ; 210$ & DuPont 951 & 20 & 15 & 1000 & DuPont 6145 & DuPont 6145 & DuPont 6146 & 5 Yes & Yes \\
\hline 5 & & $4 \sim 46.52$ & RFTF4 & -1.81 & $131 ; 121 ; 128$ & & DuPont 951 & 20 & 15 & 1000 & DuPont 6145 & ESL 903-A & DuPont 6146 & 5 Yes & Yes \\
\hline 6 & & 5 RFTF1 & RFTF5 & -1.81 & $131 ; 121 ; 128$ & $145 ; 174 ; 118 ; 136 ; 133 ; 148 ; 127$ & DuPont 951 & 20 & 15 & 1000 & DuPont 6145 & ESL 903-A & DuPont 6146 & 5 Yes & Yes \\
\hline 7 & & 6 RFTF2 & RFTF5 & -1.81 & $131 ; 121 ; 128$ & & DuPont 951 & 20 & 15 & 1000 & DuPont 6145 & ESL 903-A & DuPont 6146 & 5 Yes & Yes \\
\hline 8 & & 7 RFTFIVE1 & RFTFIVE & -1.81 & $131 ; 121 ; 128$ & & DuPont 951 & 20 & 15 & 1000 & DuPont 6145 & ESL 903-A & DuPont 6146 & 5 Yes & Yes \\
\hline 9 & & 8 RFTF3 & RFTF5 & -1.81 & $131 ; 121 ; 128$ & & DuPont 951 & 20 & 15 & 1000 & DuPont 6145 & ESL 903-A & DuPont 6146 & 5 Yes & Yes \\
\hline 10 & & 9 RFTF4 & RFTF5 & -2.23 & $106 ; 104 ; 104$ & $150 ; 128 ; 93$ & DuPont 952 & 20 & 15 & 1000 & DuPont 6145 & ESL 903-A & DuPont 6146 & 5 Yes & Yes \\
\hline 11 & 10 & 0 RFTF5 & RFTF6 & -3.08 & & & DuPont 953 & 20 & 15 & 1000 & Ferro 903-A & Ferro 903-A & DuPont 6146 & 5 Yes & Yes \\
\hline 12 & 11 & 1 RFTF6 & RFTF6 & -3.08 & & & DuPont 953 & 20 & 15 & 1000 & Ferro 903-A & Ferro 903-A & DuPont 6146 & 5 Yes & Yes \\
\hline 13 & 12 & 2 RFTF7 & RFTF6 & -3.08 & & & DuPont 953 & 20 & 15 & 1000 & Ferro 903-A & Ferro 903-A & DuPont 6146 & 5 Yes & Yes \\
\hline 14 & 13 & 3 RFTF8 & RFTF6 & -3.08 & & & DuPont 953 & 20 & 15 & 1000 & Ferro 903-A & Ferro 903-A & DuPont 6146 & 5 Yes & Yes \\
\hline
\end{tabular}




\begin{tabular}{|c|c|c|c|c|c|c|c|c|c|c|c|c|c|}
\hline \multirow[b]{2}{*}{ Row } & \multicolumn{4}{|c|}{ Lamination } & \multicolumn{3}{|c|}{ Soldering } & \multicolumn{5}{|c|}{ Testing } & \multirow[b]{2}{*}{ Notes } \\
\hline & Bottom Lamination & Top Lamination & Together Lamination & Fire Profile & SMA Ground & SMA Trace & Ground Plane & $\begin{array}{l}\text { Straight coax } \\
\text { (Ohms) }\end{array}$ & $\begin{array}{l}\text { Resistance } \\
\text { Real (Ohms) }\end{array}$ & $\begin{array}{l}\text { Resistance } \\
\text { Imaginary } \\
\text { (Ohms) }\end{array}$ & Inductance & Stability & \\
\hline 1 & & & & & & & & & & & & & Design and build \\
\hline & 23000 psi; 5 min & 3000 psi; 5 min & 3000 psi; 5 min & Standard & Solder & Solder & Silver & $54 ;-3 ; 53 \mathrm{pF}$ & $110 ; 130$ & $;-100 ;-15$ & & $20 ; 10$ & Not good \\
\hline & 33000 psi; 5 min & $3000 \mathrm{psi} ; 5 \mathrm{~min}$ & 3000 psi; 5 min & Standard & Solder & Solder & Silver & $54 ;-3 ; 53 \mathrm{pF}$ & $71 ; 32$ & $;-5 ;-34$ & $36 \mathrm{pF} ; 5 \mathrm{pF}$ & $2 ; 2$ & \\
\hline & 43000 psi; 5 min & $3000 \mathrm{psi} ; 5 \mathrm{~min}$ & 3000 psi; 5 min & Standard & Solder & Solder & Silver & $54 ;-3 ; 53 \mathrm{pF}$ & $34 ; 17$ & $17 ;-2$ & $2.9 \mathrm{nH} ; 95 \mathrm{pF}$ & $1 ; 0$ & \\
\hline & 53000 psi; 5 min & 3000 psi; 5 min & 1500 psi; 5 min; PEOX & Standard & Solder & Silver & Silver & $54 ;-3 ; 53 \mathrm{pF}$ & $49 ; 53$ & $6 ; 7$ & $1 \mathrm{nH} ; 1.2 \mathrm{nH}$ & $1 ; 1$ & \\
\hline & 63000 psi; 5 min & 3000 psi; 5 min & $1500 \mathrm{psi} ; 5 \mathrm{~min}$; PEOX & Standard & Solder & Silver & Silver & $54 ;-3 ; 53 \mathrm{pF}$ & $34 ; 37$ & $;-40 ;-23$ & $4.3 \mathrm{pF} ; 7.7 \mathrm{pF}$ & $1 ; 0$ & \\
\hline & 73000 psi; 5 min & 3000 psi; 5 min & 1500 psi; 5 min; PEOX & Standard & Solder & Silver & Silver & $53 ; 1.4 ; 241 \mathrm{pF}$ & & & & & SMA broke \\
\hline & 83000 psi; 5 min & $3000 \mathrm{psi} ; 5 \mathrm{~min}$ & 1500 psi; 5 min; PEOX & Standard & Solder & Silver & Silver & $53 ; 1.4 ; 241 \mathrm{pF}$ & $48 ; 44$ & $;-149 ;-142$ & $1.1 \mathrm{pF} ; 1.2 \mathrm{pF}$ & $2 ; 2$ & \\
\hline & 93000 psi; 5 min & $3000 \mathrm{psi} ; 5 \mathrm{~min}$ & 1500 psi; 5 min; PEOX & Standard & Solder & Silver & Silver & & & & & & SMA tabs broke \\
\hline 10 & 3000 psi; 5 min & $3000 \mathrm{psi} ; 5 \mathrm{~min}$ & 1500 psi; 5 min; PEOX & Standard & Solder & Silver & Silver & $53 ; .5 ; 85 \mathrm{pH}$ & $89 ; 67$ & $38 ; 42$ & $6.9 \mathrm{nH} ; 7.3 \mathrm{nH}$ & $+1 ; 0$ & \\
\hline 11 & 13000 psi; 5 min & 3000 psi; 5 min & 1500 psi; 5 min; PEOX & Standard & Solder & Silver & Silver & $49 ; 4.5 ; 775 \mathrm{pH}$ & $60 ; 163$ & $22 ;-142$ & $9.6 \mathrm{nH} ; 1.2 \mathrm{pF}$ & $1 ; 3$ & \\
\hline 12 & 23000 psi; 5 min & 3000 psi; 5 min & $1500 \mathrm{psi} ; 5 \mathrm{~min}$; PEOX & Standard & Solder & Silver & Silver & $48 ; 2.6 ; 444 \mathrm{pH}$ & $52 ; 48$ & $41 ; 37$ & $7 \mathrm{nH} ; 6 \mathrm{nH}$ & $1 ; 1$ & \\
\hline 13 & 33000 psi; 5 min & $3000 \mathrm{psi} ; 5 \mathrm{~min}$ & 1500 psi; 5 min; PEOX & Standard & Solder & Silver & Silver & $48 ; 2.6 ; 444 \mathrm{pH}$ & $63 ; 76$ & $70 ; 75$ & $12 \mathrm{nH} ; 13 \mathrm{nH}$ & $2 ; 3$ & \\
\hline 14 & 43000 psi; 5 min & $3000 \mathrm{psi} ; 5 \mathrm{~min}$ & 1500 psi; 5 min; PEOX & Standard & Solder & Silver & Silver & & & & & & Trace not connected \\
\hline
\end{tabular}


APPENDIX E: MATLAB 
Wave In-phase addition example

close all; clear all; clc

format compact

$\mathrm{x}=$ linspace $(0,2 * \mathrm{pi}, 100)$;

$\mathrm{y} 1=\sin (\mathrm{x})$

$\mathrm{y} 2=2 * \sin (\mathrm{x})$;

$\mathrm{y} 3=3 * \sin (\mathrm{x})$;

figure(1)

$\operatorname{plot}(\mathrm{x}, \mathrm{y} 1, \mathrm{x}, \mathrm{y} 2, \mathrm{x}, \mathrm{y} 3)$

title('Wave Addition')

ylabel('Displacement')

xlabel('Distance')

$\operatorname{axis}\left(\left[\begin{array}{ll}0 & 2 * \text { pi }-3\end{array}\right]\right)$

legend('y1 $\left.=\sin (x)^{\prime}, y^{\prime} 2=2 * \sin (x)^{\prime}, ' y 3=3 * \sin (x)^{\prime}\right)$

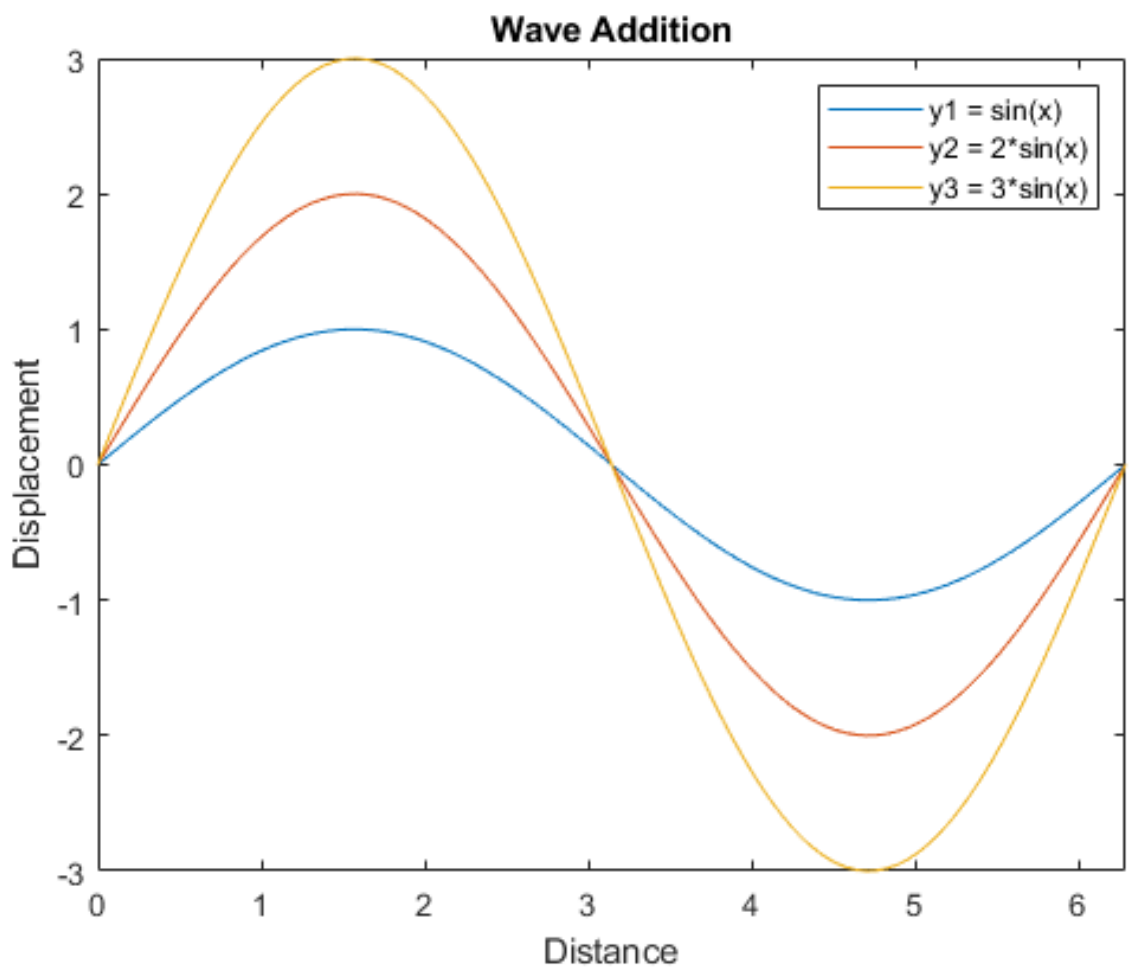

Wave out of phase addition example 
close all; clear all; clc

format compact

$\mathrm{x}=$ linspace $(0,2 * \mathrm{pi}, 100)$;

$\mathrm{y} 1=\sin (\mathrm{x})$;

$\mathrm{y} 2=\sin (\mathrm{x}+\mathrm{pi} / 2)$;

$\mathrm{y} 3=\mathrm{y} 1+\mathrm{y} 2 ;$

figure(2)

$\operatorname{plot}(\mathrm{x}, \mathrm{y} 1, \mathrm{x}, \mathrm{y} 2, \mathrm{x}, \mathrm{y} 3)$

title('Wave Addition')

ylabel('Displacement')

xlabel('Distance')

axis([0 2*pi -3 3])

legend $\left(' y 1=\sin (x)^{\prime}, ' y 2=\sin (x+\right.$ pi/2)','y3 = y1 + y2')

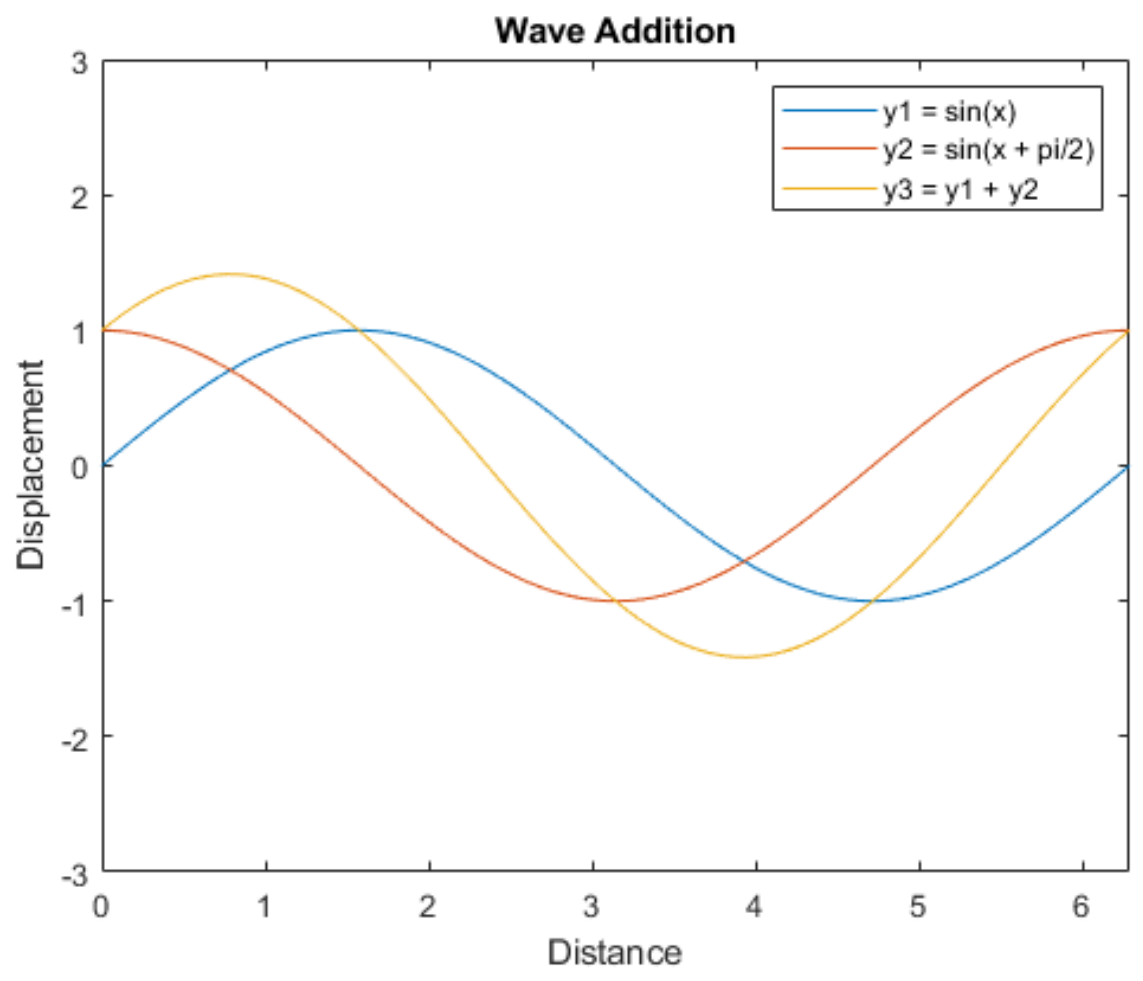

Published with MATLAB® R2018b 